\title{
Reflex sympathetic dystrophy : a clinical and experimental study
}

Citation for published version (APA):

Kurvers, H. A. J. M. (1997). Reflex sympathetic dystrophy : a clinical and experimental study. [Doctoral Thesis, Maastricht University]. Universiteit Maastricht. https://doi.org/10.26481/dis.19970523hk

Document status and date:

Published: 01/01/1997

DOI:

10.26481/dis.19970523hk

Document Version:

Publisher's PDF, also known as Version of record

\section{Please check the document version of this publication:}

- A submitted manuscript is the version of the article upon submission and before peer-review. There can be important differences between the submitted version and the official published version of record.

People interested in the research are advised to contact the author for the final version of the publication, or visit the DOI to the publisher's website.

- The final author version and the galley proof are versions of the publication after peer review.

- The final published version features the final layout of the paper including the volume, issue and page numbers.

Link to publication

\footnotetext{
General rights rights.

- You may freely distribute the URL identifying the publication in the public portal. please follow below link for the End User Agreement:

www.umlib.nl/taverne-license

Take down policy

If you believe that this document breaches copyright please contact us at:

repository@maastrichtuniversity.nl

providing details and we will investigate your claim.
}

Copyright and moral rights for the publications made accessible in the public portal are retained by the authors and/or other copyright owners and it is a condition of accessing publications that users recognise and abide by the legal requirements associated with these

- Users may download and print one copy of any publication from the public portal for the purpose of private study or research.

- You may not further distribute the material or use it for any profit-making activity or commercial gain

If the publication is distributed under the terms of Article $25 \mathrm{fa}$ of the Dutch Copyright Act, indicated by the "Taverne" license above, 


\section{Reflex sympathetic dystrophy:}

a clinical and experimental study 


\title{
Reflex sympathetic dystrophy
}

\section{a clinical and experimental study}

\author{
Proefschrift \\ ter verkrijging van de graad van doctor \\ aan de Universiteit Maastricht, \\ op gezag van de Rector Magnificus \\ Prof. Mr. M.J. Cohen \\ volgens het besluit van het College van Decanen \\ in het openbaar te verdedigen \\ op vrijdag 23 mei 1997 om $\mathbb{1 6} .00$ uur
}

door

Henricus Anna Johannes Maria Kurvers

geboren te Sittard op 31 juli 1964 


\section{Cover illustrations:}

Top: Scene from the Algerian war, captwe of the Mouma pass from Horace Vernet (1789-1863). This French painter worked in the Romantic tradition. His work often included war scenes. Military surgeons, as portrayed in this painting, frequently encounter RSD patients, obviously because of the large number of patients with injury of an extremity.

Middle: Weir Mitchell was the first" (1864) to report a large series of patients with persistent burnimg pain in an extremuty following gunshot-wound injuries of peripheral netves. His patients were casualties of the American Civil War. Mitchell's classical description represents a monumental example of devotion and attentiveness to patients' complaints with subsequent clear recording of data. Since then, fittle of significance has been added to the clinical description of RSD he presented.

Bottom: The Cry or the Scream from Edward Munch (1863-1944). This gifted Norwegian painter and printmaker played a vital role in the development of expressionism. His work often included the symbolic portray al of such themes as misery, sickness, and death. The Cry (1893) is typical in its anguished expression of pain and fear, which are frequently observed in patients with severe reflex sympathetic dystrophy. 


\section{Promotores:}

Prof. Dr. P.J.E.H.M. Kitslaar

Prof. Dr. M.J.H.M. Jacobs (Universiteit van Amsterdam)

Prof. Dr. R.S. Reneman

\section{Co-promotor:}

Dr. D.W. Slaaf

\section{Beoordelingscommissie:}

Prof. Dr. H.A.J. Struijker Boudier (voorzitter)

Prof. Dr. R.J.A. Goris

Prof. Dr. M.E. Sluijter

Prof. Dr. J.E. Tooke (Postgraduate Medical School, Exeter, Dewon, UK)

Prof. Dr. F. Spans

The investigations described in this thesis were carried out at the University Hospital Maastricht and at the Maastricht University, The Netherlands.

Financial support by the Netherlands Heart Foundation for the project (NHS, D 89008 ) and for the publication of this thesis is gratefully acknowledged.

Additional financial support for the publication of this thesis was generously provided by Medtronic Interstim and the Remmert Adriaan Laan Fonds. 
Surgeons of all men are most happy: what good success soever they have, the world proclaimeth, and what faults they committ, the earth coveret.

Adapted from F. Quarles, Hieroglyphics of the life of men (1638).

Also he must know the anatomy: for those surgeons that work in man's body not knowing the anatomy is likened to a blind man that cutteth a vine tree, for he taketh away more or lesse than he ought to doo.

From The Qualities of a Surgeon, Thomas Vicary (1490 - 1562)

In memory of my father, to my parents. 


\section{Contents}

1 Introduction 9

1.1 Neural regulation of vascular tone $\quad 10$

$\begin{array}{lll}1.2 & \text { Reflex sympathetic dystrophy (RSD) } & 12\end{array}$

1.3 Chronic constriction injury (CCI) model $\quad 15$

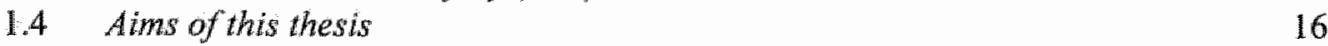

$\begin{array}{ll}1.5 . & 16 \\ 1.6 & 16\end{array}$

$\begin{array}{ll}1.6 & \text { Outline of this thesis: } \\ & 17\end{array}$

2 Reflex sympathetic dystrophy: general overview 19

$\begin{array}{lll}2.1 & \text { General introduction } & 20\end{array}$

2.2 Nomenclature $\quad 20$

2.3 Clinical characteristics of RSD 22

$\begin{array}{lll}2.4 & \text { Hypotheses on mechanisms underlying RSD } & 27\end{array}$

2.5 Studies in RSD patients 30

2.6 Therapeutical interventions for RSD 35

3 Animal models nerve injury; general overview 39

3.1 General introduction on various animal models of nerve injury 40

3.2 The chronic constriction injury (CCl) model 42

3.3 Changes in the sciatic nerve (CCI model) 44

3.4 The role of the saphenous nerve (CCI model) 48

3.5 Changes in the spinal cord (CCI model) 49

3.6 Changes in the brain (CCI model) 53

3.7 Signs and symptoms (CCI model)

$\begin{array}{lll}3.8 & \text { Therapeutical interventions (CCI model) } & 57\end{array}$

$4 \quad$ Materials and methods $\quad 59$

4.1. Reflex sympathetic dystrophy $\quad 60$

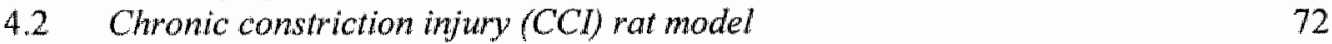

5 Reflex sympathetic dystrophy: evolution of microcirculatory disturbances in time

6 Reflex sympathetic dystrophy: result of autonomic denervation?

7 Reflex sympathetic dystrophy: the influence of local skin heating and reactive hyperaemia on skin blood flow abnormalities

8 Reflex sympathetic dystrophy: the spinal component to skin blood flow abnormalities 
9 Reflex sympathetic dystrophy: does sympathetic dysfunction originate from peripheral neuropathy?

10. Skin blood flow abnormalities in a rat model of partial nerve injury: result of decreased sympathetic vasoconstrictor outflow?

11 Skin blood flow disturbances in the contralateral limb in a rat model of partial nerve injury

12 Partial nerve injury in the rat: influence on efferent function of sympathetic and antidromically acting sensory nerve fibres

13 In vitro investigation of sympathetic dysfunction in reflex sympathetic dystrophy patients and in rats with partial nerve injury: denervation-induced supersensitivity to catecholamines

14 Summary and general discussion 161

14.1 Validity of the CCI model 162

14.2 Sympathetic dysfunction 163

$\begin{array}{ll}14.3 \text { Antidromically acting sensory nerve fibres } & 170\end{array}$

14.4 Interactions between sympathetic and sensory nervous system 173

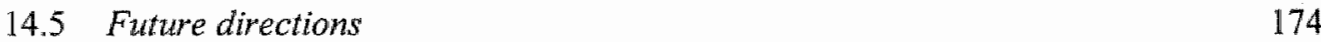

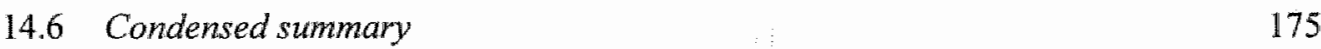

$\begin{array}{ll}\text { References } & 177\end{array}$

Korte samenvatting 197

$\begin{array}{ll}\text { Dankwoord } & 201\end{array}$

List of publications and abstracts 205

$\begin{array}{ll}\text { Curriculum vitae } & 208\end{array}$ 


\section{List of abbreviations}

$\mathrm{AChE}$

$\mathrm{CCl}$

Cde

Cdi

$\mathrm{CI}$

CGRP

CNS

CON

CPT

CRPS

CSA

CSF

CUH

D

$\Delta \mathrm{D}$

EAA

EFS

EMG

LDF

$\mathrm{I}_{\mathrm{II}} \mathrm{IG}$

LST

M

NCV

NMDA

NONLIG

NSBF

PP

$\mathrm{R}$

$\mathrm{r}$

$\mathrm{RBCV}$

$\mathrm{RD}$

RSD

$\mathrm{SBF}$

SBP

SCS

SEP

$\mathrm{SM}$

$\mathrm{SP}$

ST

TCI

Tcpos

TENS

TSBF"

UVWT

VOLF

WDR

W/L acerylcholine-esterase

chronic constriction injury

capillary density

capillary diameter

confidence interval

calcitonin gene related peptide

central nervous system

control group

cold presisor test

complex regional pain syndrome

cross sectional area

cerebrospinal fluid

clinically unaffected hand

diameter

distension

excitatory amino acid

electrical field stimulation

electromyography

laser Doppler flowmetry

ligated side

local skin temperature

average media thickness

nerve conduction velocity

N-methyl-D-aspartate

non-ligated side

nutritive skin blood flow

pulse pressure

correlation coefficient

internal lumen radius

red blood cell velocity

relative distension

reflex sympathetic dystrophy

skin blood flow

systolic blood pressure

spinal cord stimulation

somatosensory evoked potential

percentage smokers

substance P

skin temperature

transport capacity index

transcutaneous oximetry

transcutaneous nerve stimulation

total skin blood flow

ultrasonic vessel wall tracking

volume flow

wide dynamic range

wall to lumen ratio 
Chapter 1

Introduction 


\subsection{NEURAL REGULATION OF VASCULAR TONE}

\section{1.a Sympathetic nervous system (fig. 1.1)}

The portion of the nervous system that controls the visceral functions of the body is being referred to as the autonomic nervous system. It can be divided into a sympathetic and a parasympathetic subsystem ${ }^{211}$. The cell body of the preganglionic sympathetic neurons lies in the intermedio-lateral horn of the spinal cord. Its fibre passes through an anterior root of the cord into a spinal nerve. Immediately after this spinal nerve leaves the spinal column, the preganglionic sympathetic fibres leave the nerve and pass through the white ramus into one of the ganglia of the sympathetic chain. Then, these fibres may synapse with postganglionic neurons in the ganglion that it enters. From the sympathetic chain ganglia, the postganglionic fibres then travel to their effector organ. The level of activity of the sympathetic nervous system can be increased through excitation of sensory nerve fibres (somato-sympathetic reflex). The effects of sympathetic stimulation differ between effector organs. Skin blood vessels uniformly constrict upon sympathetic stimulation ${ }^{328,556}$. The effect of sympathetic stimulation on (larger) blood vessels depends on the type of adrenoceptor stimulated: constriction occurs in case of stimulation of $\alpha$-adrenoceptors whereas dilation is mediated by $\beta$-adrenoceptors and cholinergic receptors. E. g., sympathetic stimulation causes constriction and reduced distensibility of the brachial artery ${ }^{297}$.

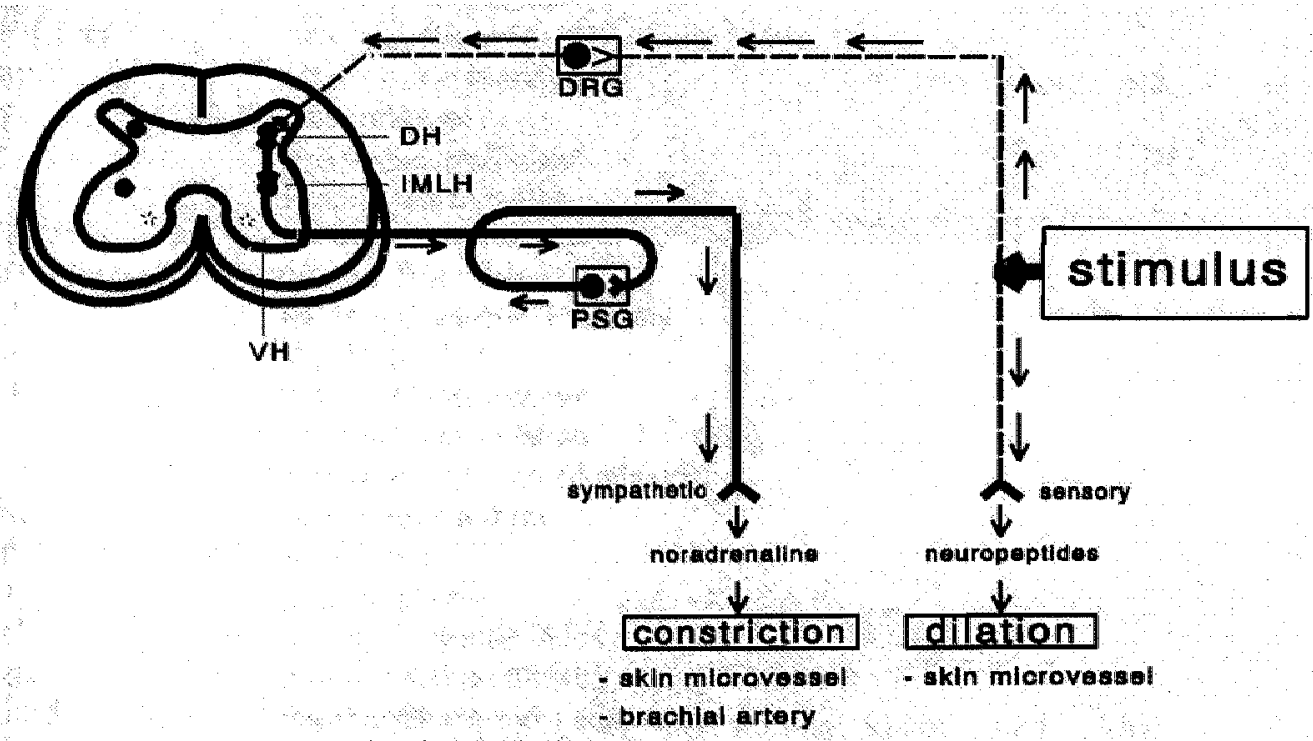

Fig. 1. 1 neural regulatony mechanisms of vascular tone goth orthodromically acting (vasoconstrictor) sympathetic and entidromically acting (vasodilator) sensory nenve fibies are indicated Excilation of sensony nene fibres sibultaneousty increases the level of efferent (vasoconstrictor) impulses in sympathetic nene fibres through somatosympathetic ireflexes as well as the level of antidromic vasodilator impulses in senson nenve fibres. DRG = dorsal root gangition $\mathrm{DH}=$ dorsal horn IMLH intermedio lateral horn, VH = ventral horn, $\mathrm{PSG}=$ paravertebral sympathetic gangllion. 


\section{1.b Sensory nervous system (fig. 1.1)}

The sensory nervous system transmits sensory information from receptors to primary sensory areas in the central nervous system from where it is relayed to essentially all other segments of the nervous system ${ }^{21}$. Following excitation of sensory receptors in the skin, impulses travel via sensory fibres in large mixed peripheral nerves towards the cell bodies in the dorsal root ganglion. From there, sensory impulses enter the spinal cord through the posterior roots from where they are being relayed to the brain. At the level of the spinal cord connections exist between sensory neurons (dorsal root) and sympathetic neurons (intermedio-lateral horn) or motor neurons (ventral horn). These connections are involved in somato-sympathetic and motor reflexes, respectively. The most important function of peripheral sensory nerve fibres is orthodromic transmission of impulses from sensory receptors to the dorsal root ganglion. Besides, electrical stimulation, tissue damage, or local axon reflexes may all induce impulses in sensory nerve fibres which are directed from the site of excitation towards the periphery. These antidromic impulses cause the release of neuropeptides at the peripheral endings of sensory nerve fibres which, in turn, may provoke inflammatory events such as vasodilation, and enhancement of vascular permeability and protein extravasation ${ }^{73}$. Collectively, these phenomena are being referred to as neurogenic inflammation.

\section{1.c Skin microvessels}

The skin microcirculation is organised in two functional subsystems. One part consists of the subpapillary arteriolar and venular plexus, interconnected by arterio-venular anastomoses, which are mainly involved in thermoregulation ${ }^{363,547}$ (fig. 1.2). The other part embodies the superficial capillaries in the dermal papillae, which supply oxygen and nutritives to the skin. The thermoregulatory subsystem is predominantly neurally controlled ${ }^{219,519}$. Increased activity/sensitivity of the sympathetic nervous system increases vascular tone, thus reducing blood flow at this level of skin microcirculation ${ }^{328,556}$. Likewise, decreased activity/sensitivity of the sympathetic nervous system increases thermoregulatory skin blood flow ${ }^{314,556}$. In contrast, activation of antidromically acting sensory nerve fibres reduces vascular tone, thus increasing thermoregulatory skin blood flow ${ }^{53,174}$. The nutritive subsystem is mainly controlled by local factors such as vasoactive metabolic substances, for example, accumulating during tissue ischemia ${ }^{519,547}$.

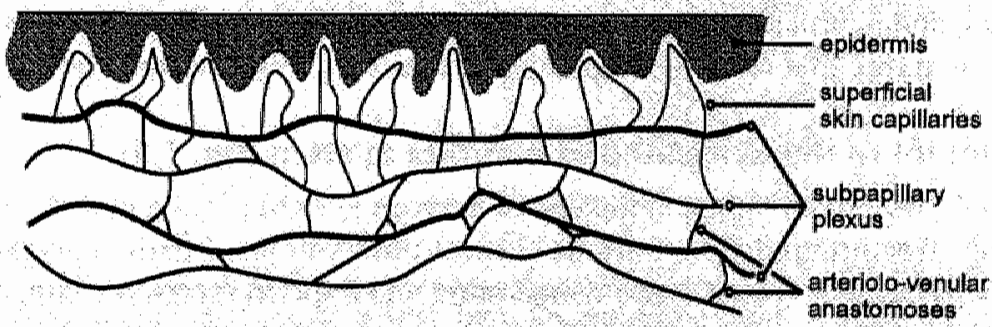

Fig. 1.2 anatomy of the skin microcirculation themoregulatory and nutritive subsystem. 


\subsection{REFLEX SYMPATHETIC DYSTROPHY (RSD)}

\section{2.a Introduction}

Reflex sympathetic dystrophy (RSD) is a neuropathic pain syndrome usually localised in an extremity and often resulting from trauma. The syndrome is associated with a large number of signs and symptoms (sensory, autonomic, motor, and trophic) which may vary extremely in presence and severity, not only between patients but also in time.

RSD is a significant problem worldwide. Its reported prevalence varies widely. Following peripheral nerve injury it has been reported to occur in 1 to 15 percent of the cases $^{1,118,445}$, whereas following wrist fractures it has been reported to occur in $0.03^{62}$ to $38^{21}$ percent of the cases. In the Netherlands, the overall annual incidence has been estimated to be around 8000 cases $^{2}$.

Numerous diagnostic criteria have been proposed for RSD. Although many underlying pathophysiological mechanisms have been proposed, the exact pathogenesis of RSD is still unclear. Consequently, RSD is often not recognised and treated inappropriately or with delay and patients may undergo various types of treatment which frequently have little rational basis. Therefore, it is not surprising that the results of these types of treatment are often not satisfactory. In patients refractory to treatment, the pain may become of truly horrifying severity and completely debilitating. Consequently, severe psychiatric disorders may develop, ranging from depression to suicidal behaviour. In extreme cases, the severity of the complaints may result in amputation of the affected limb.

These considerations attest to the necessity of further (buman and animal) research in order to unravel the mechanisms that lead to the clinical phenomenology of RSD. Insight into these mechanisms may lead to the development of more rational (and hopefully more effective) therapeutical approaches.

\section{2.b Pathophysiological mechanisms}

Although RSD has long been recognised clinically, basic research has been relatively sparse. Consequently, purported underlying pathophysiological mechanisms are often based on hypothetical considerations or derived from findings in experimental animal studies.

It is generally agreed that sensitivity and afferent activity of nociceptor-units is increased in RSD patients. Increased sensitivity/activity of the nociceptor-unit may occur at the level of skin receptors, the midaxon, or the dorsal root ganglia. The high levels of discharge originating from the affected extremity may, in turn, induce anatomical, elec trophysiological, and neurochemical changes in the spinal cord. Consequently, processing of (abnormal) input at this first relay in the pathway of sensory impulses may be altered.

\section{2.c Involvement of the sympathetic nervous system (fig. 1.3)}

It has often been purported that the sympathetic nervous system plays a pivotal role in RSD. This assumption was inferred from various clinical observations. Firstly, several signs and symptoms of RSD, such as reduced skin temperature and hyperhydrosis, are suggestive of an increased level of efferent sympathetic nerve impulses. Secondly, surgical ${ }^{394,439}$ and che mical $^{235,115}$ sympathectomy have beneficial effects on sensory abnormalities in part of the RSD patients. Collectively, these observations have led to the theory that in RSD, the initial 
preeipitating event, i.e, injury to peripheral tissues or nerves, may lead to alteration in the functioning of primary sensory neurones, either as a direct response to injury or due to changes in the milieu surrounding the afferent terminal. The increased afferent activity arising from the damaged periphery is signalled to the spinal cord where it may alter the responsiveness of sympathetic preganglionics as a result of which the rate of efferent sympathetic nerve impulses may increase (somato-sympathetic reflex) ${ }^{57,337}$. Besides, it has been proposed that injury of a peripheral nerve may increase directly the level of efferent impulses in sympathetic nerve fibres. This allegedly increased level of efferent sympathetic nerve impulses in combination with the (abnormal) responsiveness of sensory nerve fibres to. catecholamines provide an element of positive feedback, thus reinforcing or exacerbating the abnormal sensory inflow. The latter hypothesis seemingly accounts very well for the beneficial effects of sympathectomy on sensory abnormalities in part of the RSD patients. However, some signs and symptoms of RSD as well as the results of several studies in RSD patients argue against the alleged increase in the rate of efferent sympathetic nerve impulses. Firstly, especially at the early stages of RSD, the skin is often red and warm and sweat excretion may be reduced. Both phenomena suggest that at this stage efferent sympathetic nerve activity is decreased. Secondly, in RSD patients microneurographic recordings in skin fascicles of sympathetic efferents did not exhibit increased activity ${ }^{71,483,513}$. Thirdly, it has been demonstrated that venous plasma levels of noradrenaline ${ }^{138,218}$ and neuropeptide $\mathrm{Y}^{137}$ collected in the affected limb of RSD patients are decreased when compared with plasma samples obtained from the contralateral extremity.

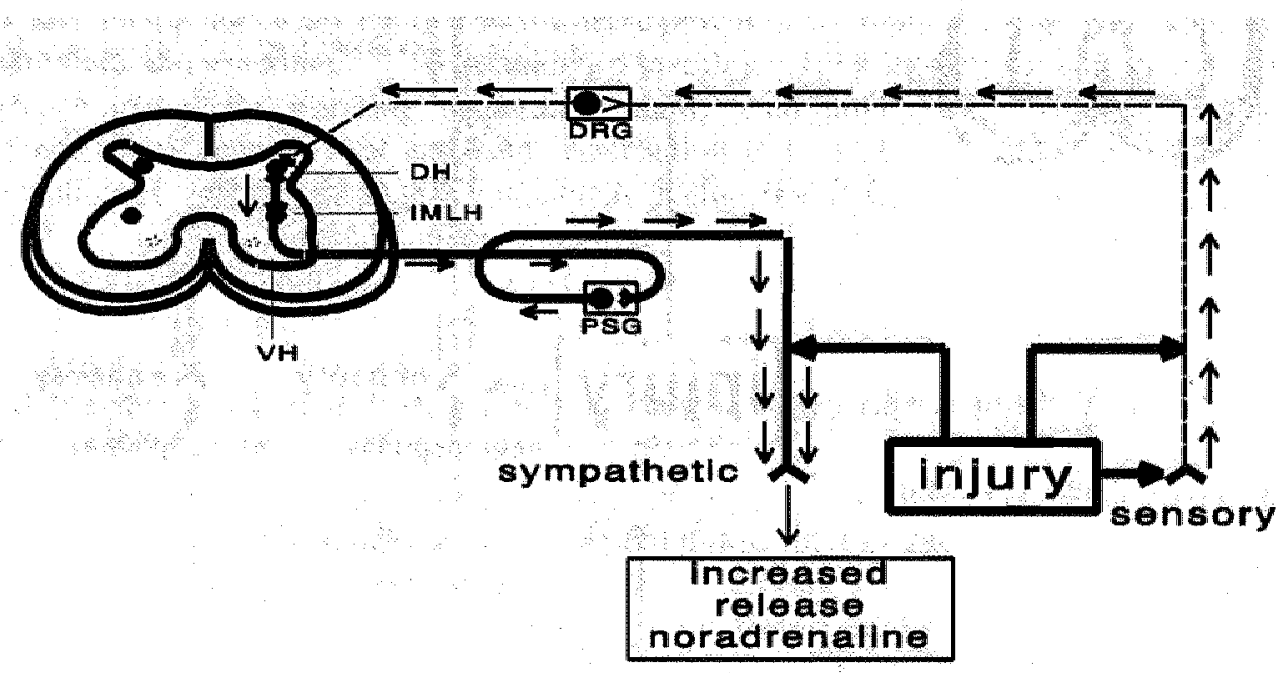

Fig. 1.3: purported pathophysiological mechanism of sympathetic dy sfunction in RSD Partial injury of a nenve either at micaxonal level of at the level of peripheral nerve twigs, provokes an increase in the level of efferent sympathetic merve impulses as a result of 1 ) abarrage of sensory impulses directed towards the central nervous system (through somato-sympathetc reflexes), and 2 ) direct excitation of sympathefic nerve fibres. $\mathrm{DRC}=$ dorsal root ganglion $\mathrm{DH}=$ dorsal horn; $1 M L H=$ intermedio-lateral horn: $V H=$ ventral hom; PSG = paravertebrall sympathetic ganglion. 


\section{2.d Involvement of antidromically acting sensory nerve fibres (neurogenic inflammation) (fig. 1.4)}

Several signs and symptoms commonly observed in RSD patients (calor, tumor, rubor, dolor, functio laesa) suggest the presence of an inflammatory process which may be mediated by antidromically acting sensory nerve fibres. Although already in 1920 Lewis ${ }^{323}$ hypothesised that RSD results from release of pain-producing vasodilator substances at the endings of sensory nerve fibres consequent to excitation of these nerve fibres somewhere at the axonal level, it is still unknown whether antidromically acting sensory nerve fibres are involved in the development and maintenance of signs and symptoms in RSD patients. Evidence in favour of the hypothesis that antidromically acting sensory nerve fibres are involved in sensory abnormalities of nerve-injured patients was provided by studies in which it was demonstrated that local capsaicin application, which locally depletes substance $P(S P)$, reduces pain in neuralgia patients ${ }^{77,449}$.

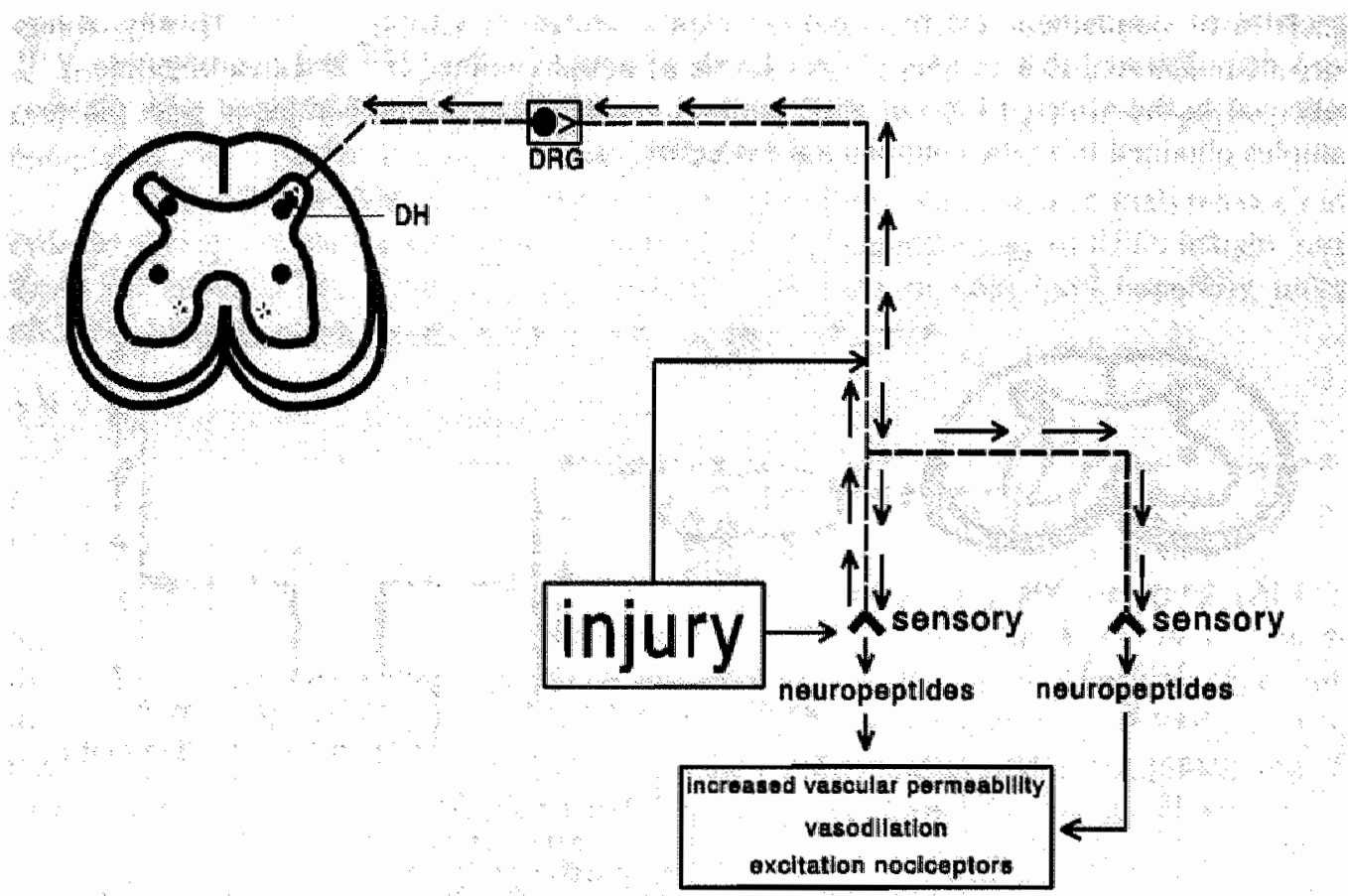

Fig. 1.4. purpoited pathop hysiological mechanism of dysfunction of sensory nerve fibres in RSD, Partial inumy of a nerve, either at midaxonal level or at the level of peripheral nenve wigs, provokes an increase in the level of both athodromically and antidromically directed impulses in sensony nerve fibres As a result of the latter neuropeptides are released at penpheral endings of sensory, newe fibres, thereby causing vasodilation, increased vascular permeablity, and excltation of nociceptors. $D R G=$ dorsal root ganglion $\mathrm{DH}=$ dorsal horn. 


\subsection{CHRONIC CONSTRICTION INJURY (CCI) MODEL}

\section{3.a Introduction}

It was not until 1988 , before Bennett and Xie ${ }^{40}$ reported on an animal model of partial peripheral nerve injury in which signs and symptoms could be induced reminiscent of those observed in RSD through loose ligation of the sciatic nerve (chironic constriction injury $(\mathrm{CCI})$ ). Among these are not only sensory abnormalities but also other features indicative of autonomic dysfunction.

\section{3.b Pathophysiological mechanisms}

Loose ligation of the sciatic nerve evokes partial and differential deafferentiation of the sciatic nerve's territory with survival of primary afferent neurons the axons of which are interrupted and formation of a neuroma-in-continuity at the site of constriction. Despite this partial and differential deafferentiation of the sciatic's nerve peripheral territory, the spinal cord dorsal horn is bombarded by high levels of discharge from many of the injured nerve's afferents which have become more sensitive to various stimuli (among others catecholamines). The increased level of afferent impulses causes release of (neurotoxic) excitatory amino acids and consequently transsynaptic degeneration in the superficial dorsal horn (both ipsi- ${ }^{493}$ and contralateral ${ }^{493}$ side).

\section{3.c Involvement of the sympathetic nervous system}

The results of several studies indicate altered activity of the sympathetic nervous system in $\mathrm{CCI}$ rats. Firstly, loose ligation of a sciatic nerve provokes dystrophic claw-growth ${ }^{40}$ and skin temperature abnormalities ${ }^{39,40,533}$. Secondly, transection of the loosely ligated sciatic nerve produces immediate warming of an abnormally cold hind paw ${ }^{39}$. Thirdly, chemical sympathectorny reduces heat and cold sensitisation in CCI rats. On the basis of these 3 observations, it was suggested that sympathetic dysfunction in $\mathrm{CCI}$ rats consists of increased efferent sympathetic nerve impulses. However, Wakisaka et al ${ }^{533}$ observed a progressive depletion of noradrenaline-containing sympathetic efferents in plantar arteries and veins, from day 5 after partial nerve injury onwards.

\section{3.d Involvement of antidromically acting sensory nerve fibres (neurogenic inflammation)}

Alike in RSD patients, it has been suggested that in the CCI model excitation of sensory nerve fibres at the site of ligation may induce antidromic impulses and consequent release of neuropeptides (such as SP) at the peripheral endings of these sensory nerve fibres. The latter phenomenon may be involved in the increase in skin temperature observed at an early stage after nerve injury as well as in the reduced pain thresholds (both locally and more widespread). Evidence in favour of the hypothesis that loose ligation of a sciatic nerve provokes increased release of substance $P$ (SP) was provided by a study of Basile et all ${ }^{32}$ who demonstrated reduced vasodilation and reduced plasma extravasation in response to SP. These reduced responses suggest down-regulation of SP receptors consequent to increased release from presynaptic sensory nerve terminals. 


\subsection{AIMS OF THIS THESIS}

In general, within the framework of this thesis we have attempted to unravel some of the pathophysiological mechanisms underlying signs and symptoms induced by partial nerve injury: we investigated how this type of nerve injury affects function of injured (vasomotor) nerve fibres (i.e., sympathetic and antidromically acting sensory nerve fibres).

It was the first and principal aim of this thesis to clarify, in both RSD patients and in CCI rats, the effects of partial nerve injury on the activity and sensitivity of the sympathetic nervous system. More specifically, we wanted to clarify whether sympathetic dysfunction consists of either an afferently-induced increase in efferent sympathetic nerve impulses or of denervation-induced supersensitivity to catecholamines. Besides, in case of denervationinduced supersensitivity to catecholamines, we wanted to elucidate whether this phenomenon consists of prejunctional (i.e., deviation) supersensitivity or of postjunctional (i.e., adaptive) supersensitivity. A second aim of this thesis was to clarify the mechanism underlying the observed phenomenon of (partial) sympathetic denervation. The latter may result from interruption of sympathetic efferents at the site of nerve injury or from dysfunction of sympathetic neurons at the level of the central nervous system and/or paravertebral sympathetic ganglia. A third aim was to clarify the effects of partial nerve injury on efferent function of antidromically acting primary sensory nerve fibres. More specifically, we wanted to clarify whether this type of nerve injury provokes antidromic (i.e., directed towards the periphery) impulses in these nerve fibres with consequent release of vasodilator neuropeptides at their peripheral nerve endings.

\subsection{METHODOLOGICAL CONSIDERATIONS}

The most direct way to study functional changes of the sympathetic nervous system would be micro-electrode recordings. However, these are difficult to calibrate ${ }^{138}$ because the signal is changed by slight adjustments to the position of the electrode tip. Positioning the electrode correctly is technically difficult and may be painful, possibly influencing efferent sympathetic nerve activity through a somato-sympathetic reflex. Moreover, this technique renders information about efferent sympathetic nerve activity but not about the sensitivity to catecholamines of effector organs.

An indirect measure of changes in activity of the sympathetic nervous system can be obtained through assessment of hemodynamic parameters. For instance, basal skin blood flow is influenced by the level of activity of the sympathetic nervous system ${ }^{219.328}$. A reduced level of basal skin blood flow suggests an increased level of efferent sympathetic nerve impulses or supersensitivity of skin microvessels to catecholamines. Similarly, an increased level of basal skin blood flow suggests a reduced level of efferent sympathetic nerve impulses. Besides, changes in skin blood flow which usually result from autonomic provocation may be used as a measure of sympathetic innervation ${ }^{219,328}$. Another hemodynamic parameter which is influenced by the level of activity of the sympathetic nervous system is the distensibility of the brachial artery: reduced distensibility suggests an increased level of efferent sympathetic nerve impulses or supersensitivity to catecholamines. Similarly, an increased distensibility 
suggests a reduced level of efferent sympathetic nerve impulses. Besides, the change in distensibility which usually results from autonomic provocation may be used as a measure of sympathetic innervation.

Since antidromic impulses in sensory nerve fibres provoke release of vasodilator neuropeptides at their peripheral endings in the skin, an indirect measure of these impulses can also be obtained through assessment of skin blood low ${ }^{53,174}$.

\subsection{OUTLINE OF THIS THESIS}

Following a historical review on RSD (chapter 2) and animal models of partial nerve injury (chapter 3), the materials and methods are described and evaluated in chapter 4. Following 5 experimental studies in RSD patients (chapters 5-9) and 4 experimental studies in CCI rats (chapters 10-13), the findings of this thesis are summarised and discussed in chapter 14.

\section{6.a Studies in RSD patients}

The goal of chapter 5 was to relate skin microcirculatory changes to clinical stage and duration of RSD. Laser Doppler flowmetry (LDF) and capillary microscopy were used to obtain information about the neurally controlled thermoregulatory and the non-neurally controlled nutritive level of skin microcirculation, respectively. Transcutaneous oximetry was employed as a measure of the oxygenation of superficial skin layers. The goal of chapter 6 was to clarify whether sympathetic dysfunction consists of an increased level of efferent sympathetic nerve impulses or of supersensitivity to catecholamines consequent to autonomic denervation. To this end, the vasoconstrictor responses upon dependency at the predominantly sympathetically controlled thermoregulatory level as well as at the nonneurally controlled nutritive level of skin microcirculation were stu-died. In case of reduced. sympathetic nerve impulses, one would expect that these responses are disturbed more alt the thermoregulatory than at the nutritive level of skin microcirculation. The goal of chapter 7 was to find out whether microcirculatory abnormalities result from functional vasospasin and/or structural wall changes of skin microvessels. To this end we studied, as a measure of the relative reserve capacity of skin microvessels, the effects of local skin heating and reactive hyperaemia on skin blood flow abnormalities. Digital blood pressure was also measured to exclude the possibility of impaired macrocirculatory blood supply. The goal of chapter 8 was to investigate whether microcirculatory changes induced by partial nerve injury are solely of peripheral origin or also have a central nervous system component. We investigated, in the clinically unaffected hand of RSD patients, basal skin blood flow and the vasoconstrictor response to dependency at the thermoregulatory and at the nutritive level of skin microcirculation. Sympathetic denervation may result from interruption of sympathetic efferents at the site of the precipitating event or from reduced efferent sympathetic nerve activity at the level of the central nervous system. We, therefore, investigated the level of activity of the sympathetic nervous system proximal and distal to the site of the precipitating event (chapter 9). To this end, we assessed distension of the brachial artery (vessel wall Doppler tracking) and skin blood flow (LDF). If sympathetic denervation results from 
interruption of sympathetic axons at the site of the precipitating event, a discongruity in sympathetic dysfunction may be expected between proximal and distal sites.

\section{6.b Studies in $\mathrm{CCl}$ rats}

The goal of chapter 10 was to investigate, by means of LDF, whether loose ligation of a rat sciatic nerve influences basal skin blood flow and/or the sympathetically mediated vasoconstrictor response to cooling of the rat abdomen in the nerve-injured hind paw. We related the time-course of these changes to the time-course of changes in skin temperature as well as to the time-course of neuropathic signs and symptoms. In order to investigate whether skin blood flow changes induced by partial nerve injury are solely of peripheral origin or also have a central nervous system component, we additionally investigated skin blood flow in the contralateral hind paw before and after chemical blockade of sensory input from the loosely ligated sciatic nerve to the spinal cord (chapter 11). To obtain more insight into the mechanisms mediating the observed vasodilator response, we additionally assessed skin blood flow in the non-ligated paw before and after chemical blockade of sensory and nonsensory nerve fibres in the non-ligated sciatic nerve. Besides, the sympathetically mediated vasoconstrictor response to cooling of the rat abdomen was assessed on the non-ligated side. The goal of chapter 12 was to explore the involvement of sensory and non-sensory nerve fibres in skin blood flow abnormalities resulting from this type of nerve injury. We, therefore, investigated skin blood flow in the hind paw before, and at an early and a later stage after loose ligation of the ipsilateral sciatic nerve. At the two time-points after ligation, skin blood flow was assessed before and after (chemical) blockade of both types of nerve fibres in the loosely ligated sciatic nerve. The goal of chapter 13 was to clarify whether sympathetic dysfunction induced by partial nerve injury consists of increased efferent sympathetic nerve activity or of supersensitivity to catecholamines. Besides, we explored whether supersensitivity to catecholamines resulted from postjunctional supersensitivity and/or from impaired presynaptic reuptake. To investigate functional aspects of sympathetic innervation and sensitivity to adrenergic agonists, we recorded in a myograph the contractile responses of arteries isolated from the hind paw of rats with 3 week old $\mathrm{CCI}$ and from amputated extremities of stage III (chronic cold sensation) RSD patients to stimulation of adrenergic nerves and exogenously administered agonists. Besides, the structural density of sympathetic nerve fibres was assessed histologically. 


\section{Chapter 2}

\section{REFLEX SYMPATHETIC DYSTROPHY:}

\section{GENERAL OVERVIEW}

HAJM Kurvers

Department of Surgery of the University Hospital Maastricht and of the Cardiovascular Research Institute Maastricht, The Netherlands.

Based on: Reflex sympathetic dystrophy: facts and hypotheses on the role of the peripheral nervous system. Journal of the Peripheral Nervous System, (in press). 


\subsection{GENERAL INTRODUCTION}

The two main causes of chronic pain syndromes in humans are partial and total peripheral nerve injury, generally caused by trauma or surgical procedures. Notwithstanding the fact that these types of nerve injury usually cause only a simple deficit in the sensations that can be evoked by stimulation of the skin, in part of the cases they may result in neuropathic pain, which may be of truly horrifying severity and completely debilitating. In part of the patients with neuropathic pain the syndrome is refractory to treatment and becomes chronic.

Although both total and partial nerve injury may induce painful and disabling neuropathies, the underlying pathophysiological mechanisms as well as the resulting signs and symptoms are quite different. Complete transection of a nerve often provokes continuous, spontaneous pain that is perceived to originate from the denervated body part. In case of amputation of a body part, the condition is called "phantom pain", whereas in case of a remaining body part the condition is called "anaesthesia dolorosa". Patients not only suffer from continuous pain, but often also from painful sensations evoked by contact with the amputation stump or adjacent regions. In patients with partial nerve injury, because of axonal degeneration of part of the sensory nerve fibres, one would expect dulling of sensation. However, many of the patients with partial nerve injury experience a profound amplification of sensation and chronic pain. Beside sensory disorders, partial nerve injury may provoke autonomic and motor disturbances.

\subsection{NOMENCLATURE}

In 1864, Weir Mitchell ${ }^{369}$ reported that gunshot-wound injuries of peripheral nerves may provoke various features of autonomic dysfunction and persistent burning pain in the affected extremity. Mitchell's patients were casualties of the American Civil War, and every war since then has been followed by clinical summaries of large numbers of cases. In this classical description, which represents a monumental example of devotion and attentiveness to patients' complaints with subsequent clear recording of data, Mitchell designated the syndrome erythromelalgia. It was not until three years later that he changed this designation into cau salgia ${ }^{367}$. Since then, little of significance has been added to the clinical description Mitchell presented.

In 1900, Sudeck, a German surgeon, reported radiologic abnormalities (patchy osteoporosis) in extremities affected by infection ${ }^{490}$. He designated the syndrome "bone atrophy". The following year, Kienbock ${ }^{276}$ related acute atrophy of bone to antecedent trauma, but it was not until 1938 that Sudeck ${ }^{498}$ accepted trauma as a precursor of the bone atrophy that now bears his name. In 1936 , Rieder ${ }^{435}$ demonstrated, through histologic examination of bone biopsies, that the bony changes observed radiologically reflect not only resorption of dead bone but also increased bone formation in adjacent areas. Besides, this author emphasised the presence of trophic changes in non-bony tissues. Consequently, this author stated that it would be appropriate to refer to "dystrophy of an extremity" rather than to "bone atrophy".

Since the first description by Mitchell ${ }^{369}$, several comparable clinical syndromes have been given different names because of either a predominant clinical feature or a precipitating 
event. Among these are minor causalgia ${ }^{235}$ (pronounced hyperaesthesia without demonistrated lesion of a large peripheral nerve), mimo-causalgia ${ }^{403}$ (precipitated by a lesion of the central nervous system), Sudecks bone atrophy ${ }^{490}$ (roentgenograms revealing patchy osteoporosis), allgoneurodystrophy ${ }^{186}$ (precipitated by minor trauma), and shoulder-hand syndrome ${ }^{484}$ or reflex neurovascular dystrophy ${ }^{485}$ (co-existent frozen shoulder). Nowadays, the term causalgia is often used to describe a symptom complex occurring after an assessed lesion of a large peripheral nerve, whereas reflex sympathetic dystrophy (RSD) is employed for patients with soft-tissue injury without established injury to a large peripheral nerve ${ }^{4 \$ 2}$ : A descriptive and operational definition of RSD was adopted at the VIth World Congress on Pain ${ }^{253}$ (1991); "Continuous pain in a portion of an extremity after trauma, which may include fracture, but does not involve a major nerve, and associated with sympathetic hyperactivity". However, various considerations argue against the use of this more traditional than useful separation. Although according to the traditional diagnosis of RSD, by definition, evidence of nerve damage is lacking, no specific clinical or electrodiagnostic criteria have been defined in order to rule out the presence of injury of a large peripheral nerve. Moreover, electrophysiological techniques, which are currently used in clinical practice, provide information solely about the conduction properties of part of the sensory and motor nerve fibres within a specific large peripheral nerve. Hence, an undisturbed electrodiagnostic study merely indicates that conduction of impulses (in part of the nerve fibres) is undisturbed. As may be inferred from the following considerations, electrophysiologic techniques are not suited to exclude dysfunction of a large peripheral nerve. Firstly, routinely used electrophysiologic techniques do not provide information on autonomic (sympathetic) nerve fibres. The latter have been purported to play an important role in the pathophysiological mechanisms underlying RSD. Secondly, it has been suggested that, through the release of a neurokinin at their terminal (peripheral) endings, antidromically acting nociceptor nerve fibres may contribute to signs and symptoms of RSD ${ }^{73}$. The presence of such a mechanism does not exclude normal conduction velocities of these sensory nerve fibres. Thirdly, determination of both motor and sensory NCV provides information on abnormalities of large (mixed) peripheral nerves, but not on terminal nerve twigs. Fourthly, the NCV determinations mainly evaluate the fastest conducting fibres and, hence, may not exhibit abnormalities when the disease affects the smaller fibres selectively ${ }^{142}$. Besides, in compression neuropathies, it is conceivable that some fascicles with fast-conducting fibres may be spared, resulting in both computation of normal conduction velocity and poor correlation with the clinical picture ${ }^{11}$. Fifthly, it has been demonstrated that an inflammation of the rat's sciatic nerve sheath (a neuritis) at midthigh level can produce hyperalgesia in the ipsilateral hind paw that lasts for about a week ${ }^{354}$. Electron microscopy confirmed that actual damage to the axons within the nerve was not necessary for the effect. It is possible to imagine that an undetected neuritis (nerve irritation from traction from operative scar tissue or from a poorly healed ligament tear) in a patient might have the same effect, without influencing NCV. The inability of electrophysiological studies to exclude nerve dysfunction may account for the not uncommon observation that physical examination in RSD patients is indicative of increased excitability (tenderness) of a large peripheral nerve, whereas conduction properties are undisturbed. Another consideration which argues against the use of the traditional separation between RSD and causalgia patients is the observation that clinical characteristics of patients with proven nerve injury do not differ from those of patients without clinical evidence of 
damage to a large (mixed) peripheral nerve ${ }^{458,504}$. The latter observation suggests that both patient groups share similar pathophysiological mechanisms. The final consideration which argues against separation of RSD and causalgia is that in both patient groups terminal nerve twigs may be damaged. Injury to a large peripheral nerve severs axons or disorganises axonal mechanism as a result of which terminal nerve twigs distal to the site of injury fail to survive ${ }^{499}$. Similarly, in patients with soft-tissue injury minuscule terminal nerve twigs may be damaged directly. Collectively, these considerations prompted us to refrain from separating causalgia and RSD: both patients with (presumed) injury of a large peripheral nerve and patients with softtissue injury were included in the studies.

The commonly used term reflex sympathetic dystrophy is based on the assumption that the clinical phenomenology is the result of a reflex activation of sympathetic neurons ${ }^{57,333,417}$. However, in RSD patients assessment of efferent sympathetic nerve activity (through microelectrode recordings) did not demonstrate increased activity ${ }^{71,483,513}$. Besides, it has been demonstrated that venous plasma levels of noradrenaline ${ }^{138,218}$ and neuropeptide $\mathrm{Y}^{137}$ collected in the affected limb of RSD patients are decreased when compared with plasma samples obtained from the contralateral (unaffected) extremity. Together, these findings suggest that the term RSD is used inappropriately. As an extension and completion of the consensus statement on RSD (199l) as well as to avoid ambiguities, a revised taxonomic system for RSD was presented in $1994^{3}$. Syndromes previously designated RSD or causalgia were classified under the neutral umbrella term complex regional pain syndrome (CRPS), which is based completely on clinical criteria. Proposed diagnostic criteria for CRPS are the presence of regional pain (spontaneous and evoked) and other sensory changes following a noxious event. The pain is associated with changes in skin colour, skin temperature, abnormal sweating, edema, and sometimes motor abnormalities. Two subtypes of CRPS have been defined: CRPS type I which corresponds to RSD, and CRPS type II which corresponds to causalgia.

Although the term CRPS, which does not allude to underlying pathophysiological mechanisms, may be more appropriate than RSD, the latter will be used throughout the text of this thesis because it will take time to replace this deeply ingrained term.

\subsection{CLINICAL CHARACTERISTICS OF RSD}

\section{3.a Precipitating events}

RSD is associated with a wide variety of precipitating factors (table 2.1). The common mechanism of these factors has been purported to be injury or disease of neural tissue ${ }^{464}$, either centrally (brain, spinal cord) or peripherally (dorsal root ganglion, large peripheral nerves, and terminal nerve twigs). The involvement of neural tissue in RSD is among others confirmed by the observation that the syndrome may develop in an extremity following injury ${ }^{443}$ or disease ${ }^{294,486}$ of the central nervous system. Obviously, neural trauma secondary to accidental (or intentional) injury of an extremity is the most common cause in surgical practice ${ }^{419}$. Nevertheless, one should realise that identical clinical features may arise when the cause of nerve damage is non-traumatic.

Several studies have addressed factors which may predispose to the development of RSD. Some studies have reported increased occurrence of RSD in some families which may 
indicate fanilial predisposition ${ }^{199}$. Mailis and Wade $^{345}$ investigated the HLA antigen profiles of Caucasian women with RSD. The results of this study suggest a possible genetic susceptibility profile in patients with refractory RSD. Genetic differences in the pathophysiological tendency of nerves of different individuals may account for the enormous variability of signs and symptoms of RSD following essentially similar injuries. An et $a^{14}$ performed a retrospective study demonstrating that cigarette smoking is increased in RSD patients when compared with hospitalised controls (68\% vs 37\%). The authors hypothesised that cigarette smoking may be involved in the pathogenesis of RSD through enhancement of sympathetic activity. In some studies, it has been reported that RSD occurs most frequently in women over the age of 50 years ${ }^{486,560}$. In other studies, the syndrome was equally distributed over all age groups ${ }^{135,399}$. The male-to-fernale ratio in these studies was approximately $2: 3$.

Considerable differences in incidence of RSD for similar precipitating events have been reported which may be related to the use of different criteria to diagnose RSD. E.g., in patients with a fracture of the distal radius some studies reported an incidence of less than $10 \%{ }^{62,168,430}$, whereas others observed development of RSD in $25 \%$ of the cases or more ${ }^{21,43,158}$. The incidence of RSD appears to be higher in prospective studies which suggests underdiagnosis in retrospective studies.

Usually, RSD is easily diagnosed because the precipitating event is an obvious trauma to a major nerve trunk, to tissues in close proximity to a major nerve trunk or to regions rich in nerve endings. However, in part of the patients RSD may be misdiagnosed because of a lack of correlation between severity of injury and severity of the resultant syndrome. In others, a precipitating event may even be untraceable ${ }^{419_{i} 484}$. In order to prevent mismanagement or neglect, one should realise that RSD indeed may be caused by seemingly minor traumas ${ }^{253,525}$. The latter is illustrated by a study of Horowitz ${ }^{238}$. who reported on 11 patients with RSD of the upper extremity in which routine venipuncture had caused injury to cutaneous nerves. The author stressed that the anatomical proximity of these nerves to veins makes them vulnerable to injury during routine venipuncture. Secondly, it is likely that in part of the RSD patients the development of the syndrome is incorrectly related to a precipitating event. The latter is illustrated by a study of Grundberg and Reagan ${ }^{202}$ who reported on 22 patients with therapyresistant RSD of the upper extremity, incorrectly related to various precipitating injuries which partly were not located at the level of the wrist. Careful reviewing of these therapy-resistant individuals revealed a carpal tunnel syndrome in all 22 patients. Besides, in 7 out of 17 patients subjected to an electrophysiologic study, the authors observed EMG abnormalities indicative of additional compression syndromes (cubital tunnel syndrome in 5, ulnar tunnel syndrome in 1 , herniated disk of the cervical spine in 1). Hence, the lack of an obvious precipitating event does not argue against the diagnosis of RSD, it merely indicates that further physical examination and/or diagnostic studies are required which may reveal the underlying cause of this syndrome.

\section{3.b Signs and symptoms}

The RSD syndrome is associated with a large number of signs and symptoms, which may vary extremely in presence and severity, not only between patients but also in time. Signs and symptoms can be categorised into 1.) sensory, 2.) autonomic, 3.) motor, and 4.) trophic disturbances. 


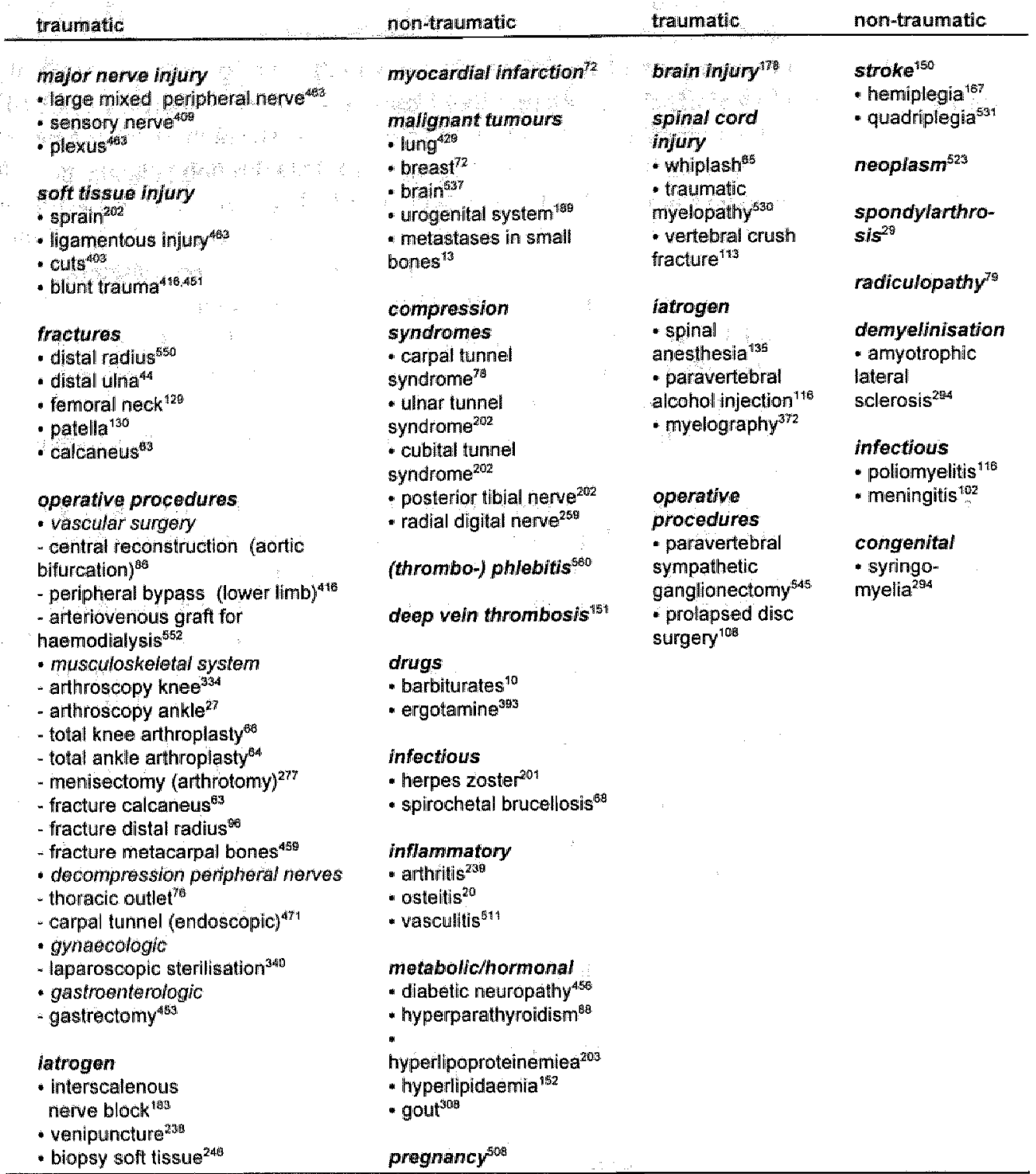

Table 2.1: events precipitating RSD, either traumatic or non-traumatic and localised in the central nerwous system or peripherally. 
For most RSD patients, stimulus-independent persistent pain ${ }^{413}$ is the most troubling feature. It is described as burning ${ }^{480}$ with a palmar or plantar predominance ${ }^{49}$, and may be out of proportion to the intiating event ${ }^{287}$. It may be limited to the distribution of a posterior root or a peripheral nerve ${ }^{313}$, which is indicative of injury to these specific neural structures.

Alternatively, pain may not be confined to the distribution of a posterior root or a peripheral nerve ${ }^{480}$. The latter may be indicative of injury to terminal nerve twigs. Besides, this spread of sensory abnomalities may be related to the observation that rats with an experimental peripheral monomeuropathy exhibit extra-territorial (outside the distribution of the lesioned nerve) sensory abnormalities ${ }^{283}$. This phenomenon has been purported to be related to a peripheral nerve injury-evoked dysfunction of pain processing neurons in the central nervous system. The pain may radiate proximally or distally ${ }^{15}$ and involve the whole ipsilateral extremity and even the contralateral extremity (mirror phenomenon) ${ }^{294}$. All together, these observations indicate that an atypical distribution of sensory abnormalities does not argue against the diagnosis of RSD. In addition to stimulus-independent persistent pain, a wide range of stimulus-induced sensory abnormalities may be present such as hyperalgesia (lowered pain threshold to noxious stimulus and enhanced pain perception), allodynia (pain elicited by a normally non-noxious stimulus), hyperpathia (delayed overreaction and after sensation to a stimulus $)^{528}$, and slow temporal summation of mechanical allodynia ${ }^{413}$. Hyperalgesia and allodynia may result from thermal ${ }^{4 / 3}$ as well as mechanical ${ }^{4 / 3}$ stimuli. Hyperaesthesia (increased sensitivity) ${ }^{528}$, hypaesthesia ${ }^{528}$ (decreased sensitivity, numbness), and paraesthesia (tingling) ${ }^{202}$ have also been reported. All of the aforementioned sensory abnormalities may be exacerbated by $\operatorname{stress}^{419}$, exercise ${ }^{71}$, or cold ${ }^{71}$.

Autonomic dysfunction in RSD patients may be characterised by abnomalities of colour (red, pale, or cyanotic) ${ }^{57}$ and temperature (warm or cold) ${ }^{528}$ of the skin. Besides, these parameters may demonstrate short-term variability ${ }^{528}$. Less frequently, the excretion of sweat is changed (anhydrosis or hyperhydrosis) ${ }^{127}$. Another important feature of RSD is edema ${ }^{463}$, which may be observed at the site of injury as well as distal to $i^{51}$ and which may increase following dependency of the affected extremity.

The motor disorder of RSD may consist of limited range of active movement ${ }^{528}$, weakness ${ }^{528}$, rapid fatiguability ${ }^{528}$, dystonia ${ }^{256}$, paresis ${ }^{528}$, and altered reflexes ${ }^{463}$. Some patients develop involuntary movement (abnormal muscle spasms ${ }^{528}$ or tremor ${ }^{568}$ ) of a limb.

In RSD patients, nail and hair may exhibit an abnormal growth rate ${ }^{29}$ as well as an abnormal consistency (brittle nails with ridges ${ }^{355}$ and coarse hair ${ }^{355}$ ). The skin may become smooth and glossy ${ }^{412}$, with decrease or loss of normal wrinkles and creases. Rarely, skin pigment changes and/or ulcers are observed ${ }^{551}$. Trophic changes of other tissues may also occur. These include atrophic changes of subcutaneous tissues (pencil point or tapered digits) ${ }^{235}$, and muscles (muscular wasting) ${ }^{528}$. At end-sitages of the syndrome, contractures of tendons often occur ${ }^{57}$ and joints may become ankylosed ${ }^{57}$.

\section{3.c Course}

The onset of RSD has been reported to vary considerably. When provoked by external trauma, the onset is usually rapid, but the onset of symptoms may vary from immediately to several weeks after injury. When the initiating event is non-traumatic, the onset may be slower and more insidious 419 . 
The clinical course of RSD has mostly been divided into three stages: the first (acute or hyperaemic), second (dystrophic or ischemic), and third (atrophic) stage $e^{60,419,464}$. The various authors do not report identical signs and symptoms at each stage of RSD. Moreover, signs and symptoms of RSD may demonstrate extreme short-term variability. Nevertheless, a general pattern in the progression in stages may be distinguished. During the first (acute or hyperaemic) stage the skin of the affected extremity is usually red and warm. The most troubling feature, however, is pain, usually of a burning nature. Almost invariably stimulus-dependent sensory abnormalities are also present. Edema, which may be observed at the site of injury as well as distal to it; is ordinarily most pronounced at this stage. Towards the end of the first stage, the skin may become smooth and taut, with decrease or loss of normal wrinkles and creases. In the second (dystrophic or ischemic) stage, the skin of the affected extremity may become moist, cold, and pale (or even cyanotic), although short-term fluctuations of skin temperature (from abnormally cold to abnormally warm and vice versa) have been reported. There is spreading of edema, increasing stiffness of the joints, and muscular wasting. Burning pain remains the major symptom. It may radiate proximally or distally and involve the whole extremity. Stimulus-induced sensory abnormalities are usually more pronounced in the second than in the first stage. The hair may become coarse, and the nails may show ridges and become brittle. The third (atrophic) stage is reported to be characterised by an ongoing cold sensation as well as marked trophic changes that eventually may become irreversible. Pain may be less prominent, but has also been reported to spread further and cripple the patient. Muscles, tendons, subcutaneous tissues, skin, and skin appendages may show increasingly trophic changes. There is extreme weakness and limitation of motion in virtually all of the involved joints, which finally may become ankylosed. Although the duration of each stage is indicated to vary from 3 to 6 months $^{486}$, the actual length may vary considerably lasting anywhere from weeks to years $^{464,462}$.

\section{3.d Diagnostic criteria}

Albeit that over the years in several experimental human studies various diagnostic criteria for RSD have been proposed, presently no consensus exists. Standardisation of diagnostic criteria would enhance the possibility to compare the results of studies in RSD patients.

For experimental studies, the criteria proposed by Kozin et al ${ }^{295}$ have often been used. These authors proposed to refer to "definite RSD" in case of pain, tenderness, vasomotor instability, and swelling (dystrophic skin changes usually present); "probable RSD" in case of pain, tenderness, and either vasomotor instability or swelling (dystrophic skin changes often present); and "possible RSD" in case of vasomotor instability and/or swelling, without pain (dystrophic skin changes occasionally present). In 1991, the American Association for Hand Surgery proposed simplified criteria: in case of diffuse pain, loss of function, and objective evidence of significant autonomic dysfunction, patients were considered to suffer from RSD ${ }^{12}$. In a prospective study on signs and symptoms of 829 RSD patients, Veldman et al ${ }^{528}$ proposed the following criteria to meet the diagnosis of RSD: 1) 4 out of the 5 following signs and symptoms: unexplained diffuse pain, difference in skin colour relative to other limb, diffuse edema, difference in skin temperature relative to other limb, limited active range of motion;2) occurrence or increase of signs and symptoms after use; 3) signs and symptoms present in an extremity and in an area much larger than the area of primary injury or operation and including 
the area distal to the primary injury. In 1993, a Special Consensus Workshop was held to revise the criteria related to RSD and causalgia ${ }^{482}$. In both RSD and causalgia, there should be (or have been) evidence of edema, skin blood flow abnormalities, or abnormal sudomotor activity in the region of the pain since the inciting event. Finally, no other conditions should be present that may account for the degree of pain and dysfunction. RSD was considered to develop after an initiating noxious event with presence of spontaneous pain or allodynia/hyperalgesia, not limited to the territory of a single peripheral nerve, and disproportionate to the inciting event. Causalgia was considered to develop after nerve injury, with presence of spontaneous pain or allodynia/hyperalgesia, not necessarily limited to the territory of a single peripheral nerve.

\section{3.e Differential diagnosis}

In various other diseases, signs and symptoms may occur alike those observed in RSD patients. Raynaud's phenomenon is used to describe clinical symptoms of symmetrical, triphasic discolouration of hands and fingers ${ }^{249}$. This phenomenon may exist without or with underlying (systemic auto-immune) disease, in which case it is classified as primary or secondary, respectively ${ }^{248}$. Raynaud's phenomenon may be distinguished from RSD because of its symmetrical distribution as well as its typical triphasic pattern of skin blood flow abnormalities. Besides, secondary Raynaud's phenomenon is characterised by morphological abnormalities of nail fold capillaries in the affected extremities, which are not present in RSD ${ }^{248}$. Acrocyanosis is characterised by an uni or bilateral permanent cyanosis of hands and fingers, without the existence of an underlying disease. Asphyxia manus et digitorum is depicted by a local, sharply bordered discolouration of one or more fingers. Acrocyanosis and asphyxia manus et digitorum may be distinguished from RSD because the latter usually is preceded by some kind of trauma. Besides, in RSD various features may be present which are not observed in acrocyanosis or asphyxia manus et digitorum such as abnormal nail or hair growth and abnormal sweat excretion. In patients presenting with pain, cold sensation, and trophic changes of the skin one should also consider the presence of limb ischemia secondary to atherosclerotic, narrowed major arteries ${ }^{505}$. Beside physical examination, several diagnostic tests, such as angiography ${ }^{455}$ and Doppler ultrasonography ${ }^{286}$, are available in order to exclude the presence of major artery disease. Pain and inflammatory signs related to rheumatologic abnormalities may be another source of diagnostic difficulties ${ }^{133}$, especially when these are present unilaterally.

\subsection{HYPOTHESES ON MECHANISMS UNDERLYING RSD}

Many hypotheses have been proposed to explain the development of RSD, although none of these may account for all signs and symptoms. In some review papers ${ }^{419,458}$, these hypotheses have been categorised according to the site (peripheral tissue or central nervous system) of the alleged underlying pathophysiological mechanism. However, one should realise that changes at the level of peripheral tissues may induce abnormalities at the level of the central nervous system. Nevertheless, for reasons of clarity we have also used this categorisation system. 


\section{4.a Peripheral tissues}

The hypothesis that peripheral tissues (including terminal nerve twigs) are involved in the initiation and progression of RSD is strongly supported by several observations. Firstly, the RSD syndrome usually results from injury to peripheral tissues ${ }^{464}$. Secondly, in case of peripheral nerve injury, especially in the early stages, symptoms of RSD are experienced within the territory of that peripheral nerve ${ }^{381}$. Moreover, in these cases local anaesthesia of the damaged nerve typically transiently abolishes the pain ${ }^{458}$.

Leriche ${ }^{220}$ hypothesised that in RSD patients vasoconstriction at the site of injury, resulting from abnornal vasomotor reflexes, leads to tissue ischemia and pain. In contrast, Lewis ${ }^{323}$ postulated that RSD is a state of painful vasodilation resulting from release of painproducing vasodilator substances in response to antidromic impulses (directed towards the periphery) in nociceptor nerve fibres, arising from the area of nerve injury. Doupe et al ${ }^{127}$ refined and extended this theory. These authors suggested that sensory abnormalities in patients with nerve injury result from excitation of sensory fibres by sympathetic efferents. According to this theory, artificial synapses or ephapses are created at the site of nerve injury consequent to deterioration of the normal insulation between adjacent fibres. Consequently, efferent sympathetic nerve impulses may cross over to sensory fibres, in which these impulses can be directed either orthodromically (towards the central nervous system) or antidromically. Impulses directed orthodromically may give rise to pain, whereas impulses directed antidromically may provoke vasodilation and decreased thresholds of peripheral nociceptors through the release of a neurokinin ${ }^{73}$. Thus, antidromic impulses in sensory nerve fibres may initiate generation of painful impulses in adjacent (non-severed) sensory nerve fibres. The interaction between sympathetic and sensory nerve fibres may also account for the observation that factors which increase the level of activity of the sympathetic nervous system, such as emotional stress and anxiety, aggravate sensory abnormalities in RSD patients ${ }^{419}$. Besides, this interaction may account for relief of complaints observed in part of the RSD patients after sympathetic blockade ${ }^{475,485}$. Finally, this concept may account for trophic and vasomotor abnormalities of the skin; afferent impulses in sensory nerve fibres may cross over to sympathetic nerve fïbres, in which these impulses can be directed or tho dromically (towards the periphery), thus giving rise to the aforementioned abnormalities. The theory of Doupe et al ${ }^{127}$, however, can not explain either pain relief by blocking of the nerve distal to the site of injury (i.e., between the alleged artificial synapse and the area of pain) or persisting sensory abnormalities in cases in which the injured nerve is completely sectioned ${ }^{56,360}$. Drucker et al ${ }^{135}$ slightly modified the theory proposed by Doupe et al ${ }^{127}$. They observed that RSD results not only from clinically assessable nerve injury but also from minor soft tissue injury. In both instances, signs and symptoms were alike and responded well to sympathectomy. Based on these observations, the authors suggested that in patients with soft-tissue injury, minuscule terminal nerve twigs are damaged and develop artificial synapses alike those previously suggested to develop in injured large peripheral nerves ${ }^{127}$. The ensuing transmission of impulses from efferent sympathetic nerve fibres to sensory nerve fibres increases the input to the spinal cord and thus the activity of the internuncial neuron pool. The latter may stimulate anterolateral sympathetic efferents, thus causing a further increase in the activity of the peripheral ephactic synapse. Consequently, a vicious circle of increased sensory and sympathetic nerve impulses is initiated. 
In 1938, Sudeck accepted trauma as a precursor of the bone atrophy that now bears his name ${ }^{491}$ In 1942, this author suggested that the radiologic changes observed in RSD reflect $a$ pathological evolution of an in principle physiologic inflammatory process ${ }^{492}$. In line with the hypothesis of Sudeck, Goris et al ${ }^{194}$ suggested in 1987 that RSD results from an excessive untoward local inflammatory response to trauma. This hypothesis was based on various observations. Firstly, most symptoms during the early stages of RSD (1.e., rubor, calor, dolour, tumour, and functio laesa) are similar to those of an inflammatory process, and occur also in areas remote from the primary injury, and increase in severity by exercising the affected extremity. Secondly, Oyen et $a^{398}$ demonstrated, by means of scintigraphy with Indium ${ }^{1 / 1}$ labelled immunoglobulin $\mathrm{G}$, increased vascular permeability in early RSD. A similar phenomenon has previously been demonstrated in infectious and inflammatory lesions ${ }^{16 !}$. Thirdly, Heerschap et al ${ }^{221}$ demonstrated, by means of ${ }^{31} \mathrm{P}$ NMR spectroscopy, impaired high energy phosphate metabolism in RSD patients, which may result from cellular hypoxia or impaired oxygen extraction. The presence of the latter phenomenon in RSD is supported by observations of increased venous oxygen saturation in $\mathrm{RSD}$ patients ${ }^{195488}$. The alleged inflammatory response was suggested to result from continuous production of toxic oxygen radicals, resulting in destruction of the various tissues in the affected extremity. The hypothesis of toxic oxygen radicals is inferred from studies demonstrating relief of signs and symptoms of RSD following administration of hydroxyl radical scavengers ${ }^{193,312}$.

\section{4.b Central nervous system}

The involvement of the central nerwous system in $\mathrm{RSD}$ is demonstrated by the initiation of signs and symptoms of RSD consequent to insults to the central nervous system ${ }^{167,373}$. Besides, in those cases in which RSD results from injury to peripheral tissues, ongoing sensory input may provoke abnormalities at the level of the spinal cord, which consequently may respond abnormally not only to sensory input from an extremity, but also to brain stem and cortical influences. These spinal and supra spinal mechanisms have been purported to be responsible for the centralisation of sensory abnormalities, as a result of which these abnormalities may become less amenable to treatment.

Some authors have speculated on the results of disproportional loss of thick myelinated nerve fibres in injured peripheral nerves. Noordenbos ${ }^{350}$ stated that in high-velocity missile wounds, the thick myelinated nerve fibres are subjected to relatively more damage than poorly myelinated or unmyelinated nerve fibres. As a result, an imbalance arises between an increased number of pain impulses conducted by unmyelinated nerve fibres on the one hand, and reduced inhibiting activity from myelinated fibres on the other. As a result of this imbalance, transmission of (the increased number of) pain impulses to the spinal cord may be facilitated. Melzack and Wall ${ }^{361}$ refined this theory. They suggested that second-order neuron cells in the substantia gelatinosa modulate sensory input. Impulses from large (A-B) sensory fibres stimulate the second-order neuron shortly and subsequently inhibit it as a result of which spinal cord transmission of sensory impulses is inhibited. In contrast, small (A-ô and C) fibre input is believed to facilitate spinal cord transmission of sensory impulses. This balance of input could be disturbed by a number of pathological processes, including soft tissue or large peripheral nerve injury. Sensory abnormalities in RSD were associated with sellective damage of large myelinated fibres, permitting the balance to fawour small fibre activity, thus opening 
the gate and increasing the transmission of painful afferent impulses. This hypothesis was referred to as the gate-control theory.

Livingston proposed the vicious circle theory ${ }^{333}$. He suggested that injury to terminal nerve twigs or a large peripheral nerve provokes constant volleys of noxious impulses. Consequently, the normal functioning of the internuncial pool becomes disturbed as a result of which abnormal firing patterns are initiated and closed self-exciting neuronal loops are generated in the dorsal horn of the spinal cord. Sunderland ${ }^{497}$ slightly modified Livingston's theory of selfsustaining abnormal internuncial pool activity. He suggested that in RSD injury to efferent sympathetic nerve fibres may induce retrograde changes in the sympathetic ganglia and transsynaptic degeneration in the spinal cord. Consequently, the function of whole groups of neurons in the spinal cord might become impaired, which could then form self-sustaining circuits. This hypothesis was referred to as the turbulence theory.

\subsection{STUDIES IN RSD PATIENTS}

\section{5.a Diagnostic studies}

The ability to diagnose RSD depends primarily on clinical assessment. Various clinical investigations may be of help in confirming the diagnosis, determining stage and severity, and yielding objective baseline data in order to monitor the patients' response to therapy.

There are no consistent routine laboratory findings in RSD patients although the erythrocyte sedimentation rate has been reported to be increased in $70 \%$ of all individuals ${ }^{150}$. Others have reported that erythrocyte sedimentation rate and leukocyte counts are normal ${ }^{196}$.

Roentgenographic studies were the first to confirm the diagnosis of RSD. In 1942, Herrmann et $a^{227}$ depicted a "posttraumatic painful osteoporosis", characterised by patchy demineralisation of the epiphyses of long bones and also of the bones of hands and feet. Soft tissues may be swollen and reticulated in appearance. Fine-detail roentgenography reveals subperiostal bone resorption, striation, and tunnelling in the cortices, as well as large excavations and tunnelling of the endosteal surface ${ }^{179,293}$. In 1980 , Helms et al ${ }^{222}$ described roentgenologic findings alike those previously depicted by Herrmann et al ${ }^{227}$. However, one should bear in mind that in RSD patients patchy demineralisation is generally not appreciated until the syndrome advances to the second stage ${ }^{154}$. Besides, the aforementioned changes are not specific for RSD ${ }^{179,293}$. Sensitivity and specificity of roentgenography in RSD patients have been reported to be $69 \%$ and $71 \%$, respectively ${ }^{295}$.

$\mathrm{RSD}$ is associated with injury to either central or peripheral neural tissue. Consequently, one would expect that electromyography (EMG) as well as assessment of nerve conduction velocity (NCV) and somatosensory evoked potentials (SEPs) may be of diagnostic use. Jupiter et $\mathrm{al}^{259}$ observed electro physiologic evidence of dysfunction of peripheral nerves in 8 out of $9(89 \%)$ RSD patients. In line with these observations, Monsivais et al ${ }^{371}$ reported on 35 RSD patients of which 30 had single or multiple nerve compressions. Of these 30 patients, $27(90 \%)$ demonstrated EMG or NCV abnormalities. Grundberg and Reagan ${ }^{202}$ reported that of 17 patients with therapy resistant RSD, $15(88 \%)$ demonstrated EMG and/or NCV abnormalities indicative of carpal tunnel syndrome. Besides, in 7 out of these 15 patients the authors observed EMG abnormalities representative of additional compression syndromes (cubital tunnel 
syndrome in 5 , wlnar tunnel syndrome in 1 , herniated disk of the cervical spine in 1). Doury $2 *$ obserwed reduced NCV in 9 out of $17(53 \%)$ RSD patients, whereas Uematsu 22 detected reduced NCV or an abnormal EMG (fibrillation potentials) in only 5 of 32 (16\%) RSD patients. Folkerts et al ${ }^{165}$ observed EMG abnormalities indicative of llesions of the peripherall motor neuron in 3 out of $3(100 \%)$ RSD patients. Other studies failed to demonstrate electrophysiologic evidence of nerve dysfunction in RSD patients ${ }^{11722,4}$. Hyman et al compared median nerve somatosensory evoked potentials between subjects with and without RSD of the upper extremity. Besides, these authors monitored the influence of stellate ganglion blockade on SEPs in RSD patients. No differences were observed in SEPs between both groups, neither before nor after stellate ganglion blocks. In conclusion, the proportions of RSD patients in which electrophysiologic abnormalities of peripheral nerves were demonstrated differ considerably between various studies. Several factors may account for this variability Firstly, there are considerable differences between these studies in criteria used to diagnose RSD as well as in the mean duration of the RSD syndrome at the time of investigation. Secondly, this variability may relate to the previously referred inability of electrophysiological studies to exclude dysfunction of a nerve: they only provide information about conduction velocities of part of the sensory and/or motor nerve fibres within a large peripheral nerve.

Several studies have demonstrated skin temperature abnormalities in RSD patients, although no relationship was observed with the duration of the RSD syndrome ${ }^{327,404}$. In these studies, skin temperature was considered to be an indirect measure of skin blood flow.

Another technique to study flow characteristics in the extremities of RSD patients is the three phase bone scanning technique, which provides information on arterial inflow, vascular volume, and bone metabolism ${ }^{353}$. It was demonstrated that the total extremity perfusion is increased at stage ${ }^{92}$, normalised at stage $\mathrm{I}^{92}$, and decreased at stage III ${ }^{92}$. The sensitivity and specificity in RSD patients have been reported to be $60 \%$ and $86 \%$, respectively 295 .

\section{5.b Histopathological findings}

In 7 patients, Webster et al ${ }^{551}$ investigated skin changes in biopsies obtained from areas affected by RSD. Light microscopy revealed stasis dermatitis in 3 patients and a type of lesion similar to pigmented purpura in 2 patients. In a biopsy obtained from an RSD patient with recurrent bullae, electron microscopy revealed a disrupted basement membrane and abnormal anchoring fibrils. Synovial biopsies, obtained by open surgical technique (under local anaesthesia) front the affected joints of 4 RSD patients, all demonstrated histological abnormalities when compared with tissues obtained from age-matched fresh cadavers ${ }^{294}$. These abnomalities consisted of varying degrees of synovial edema, proliferation and disarray of synoviall lining cells, proliferation of capillaries, fibrosis of the subsynovium, and slight perivascular infiltration with chronic inflammatory cells (chiefly lymphocytes). Kirsch ${ }^{285}$ performed lightmicroscopic studies in skeletal muscle of RSD patients. He observed edema between muscle fibres, perimysial connective tissue and connective tissue septa and degenerative changes of skeletal muscle. Histological examination of muscular blood vessels revealed intimal vacuolisation, perivascular edema, and edematous changes of the vessel wall. Folkerts et al ${ }^{165}$ investigated muscle biopsies obtained from 3 RSD patients. In all biopsies, choline-esterase reactions were reduced and pathological changes of motor end-plates were observed, whereas staining of the adrenergic part of the autonomic nervous system disclosed reduced and 
pathological adrenergic inmervation. Lagier ${ }^{306}$ studied a bone biopsy obtained from the lateral condyle of the knee of a patient suffering from RSD for 1 month. Histological examination revealed signs of active remodelling of both the cancellous bone and the (very thin) cortex. The pre-existing trabeculae of the cancellous bone showed signs of erosion by osteoclasts and lamellar bone formation by osteoblasts. The marrow was edematous and fibrillar with dilatation of capillaries: Dunstan et al ${ }^{141}$ also studied bone biopsies obtained from the left femur and tibia of a patient suffering from RSD for 5 months. Histological findings in the femur biopsy were in line with those previously reported by Lagier ${ }^{306}$. normal resorption of dead bone and adjacent areas demonstrating increased bone formation. Histologically, there was no evidence of asteoporosis. Interestingly, the biopsy from the ipsilateral tibia did not demonstrate any abnormalities, which indicates that histological abnormalities do not result from inactivity of the affected extremity.

\section{5.c Experimental studies}

\section{Afferent neural mechanisms}

Sensory abnormalities in RSD patients may include stimulus-independent as well as stimulusinduced sensory abnormalities. In recent years, various studies in nerve-injured patients as well as in normal human volunteers with experimental skin injury have provided insight into the pathophysiological mechanisms underlying these sensory abnormalities. To understand these pathophysiological mechanisms, one should realise that acute pain results from excitation of nociceptors with thin myelinated or unmyelinated axons ${ }^{516}$.

In most nerve-injured patients, stimulus-independent pain is mediated by nociceptive primary afferents as may be inferred from the observation that this type of pain is significantly reduced by local anaesthetic blockade of damaged peripheral nerves ${ }^{275}$ or affected $\operatorname{skin}^{446}$. Besides, stimulus-independent pain persists during differential blockade of conduction in myelinated non-nociceptive afferents ${ }^{67}$. Finally, different levels of ongoing nociceptor activity correlate with different intensities of pain ${ }^{198}$. The exact cause of this spontaneous activity in nociceptors remains a matter of debate. The results of experimental animal studies suggest that this phenomenon may be related to 1) de novo expression or upregulation of sodium channels or 2) a depression of potassium conductances ${ }^{12 !}$.

Hyperalgesia to heat occurs only occasionally in RSD patients. Little is known about its relation with nerve-injury. It has been suggested that, alike in patients with inflammatory pain, hyperalgesia to heat results from sensitised nociceptors ${ }^{289}$ :

It is generally agreed that in part of the RSD patients, sensory abnormalities result from or are maintained by increased sensitivity of nociceptors to catecholamines. This assumption is based on several observations. Firstly, chemical and surgical interruption of efferent sympathetic nerve impulses reduces ongoing pain and hyperalgesia ${ }^{59,335,458}$. Secondly, although both sympathetic activation and intradermal injection of noradrenaline normally do not induce pain or hyperalgesia in man or animals ${ }^{322,544}$, injection of noradrenaline into the asymptomatic limb of a former RSD patient (cured by sympathectomy) rekindled pain and hyperalgesia ${ }^{513,544}$. Similarly, iontophoresis of adrenergic agents into the skin of extremities affected by RSD may induce pain $^{544}$. Thirdly, visual and auditory stimuli, emotional disturbance, or thermal stress all cause sympathetic arousal and all may intensify pain in RSD patients ${ }^{333,335}$. The finding that 
during a differential blockade of myelinated fibres sympathetically mediated pain is still experienced ${ }^{5 / 4}$ attests to the involvement of unmyelinated fibres in the mediation of this type of pain. Several hypotheses exist as to the pathophy siological mechanisms underlying increased catecholamine sensitivity. Among others, it has been suggested that a general increase in excitability of nociceptors causes preexisting adrenoceptors to become effective ${ }^{121,122}$. Alternatively, it has been purported that injured neurons start to express adre no ceptors ${ }^{357,406}$.

Various studies have demonstrated that touch-evoked pain is not mediated by nociceptors but by sensitive mechanoreceptors with large myelinated axons that normally mediate non-painful tactile stimuli. Firstly, differential blockade of large myelinated nonnociceptive afferents abolishes brush-evoked pain ${ }^{67}$. Secondly, reaction-time measurements indicate that brush-evoked pain is mediated by fast-conducting myelinated fibres ${ }^{170}$. At the level of the dorsal horn of the spinal cord, tactile sensations have to be encoded to touchevoked pain. This abnormal encoding has been demonstrated to relate to persistent nociceptor input $^{311}$. Thus, persisting discharge of sensitised nociceptors evokes not only pain but also causes sensitisation of central pain-signalling neurons to inputs from normal mechanoreceptors. These nociceptor-induced abnormalities at the level of the CNS are very dynamic which suggests that central sensitisation remains malleable even after years of chronic pain.

In nerve-injured patients, hyperalgesia to pressure is mediated by unmyelinated nociceptive afferents ${ }^{89}$ and is strictly confined to the region of tissue injury "The latter observation indicates that it is very unlikely that a central sensitisation is solely responsible for hyperalgesia to pressure. It has been purported that this phenomenon may also be related to an increase in the size of mechanical receptive fields in a subpopulation of cutaneous nociceptors ${ }^{428}$. Such an increase would provoke a spatial summation of nociceptive inputs because more nociceptors will be activated by a given stimulus. Besides, it has been suggested that hyperalgesia to pressure involves activation of a novel class of sensory neurons that do not respond to transient noxious stimuli, but which following tissue inflammation respond vigorously and remain active for many hours ${ }^{290}$.

The pathophysiological mechanism underlying cold hyperalgesia may differ between nerve-injured patients with either cold hypaesthesia or with normal sensitivity to innocuous cold. In the former group, a preferential loss of myelinated fibres, which can inhibit the effect of nociceptor stimulation at a central level ${ }^{4.5}$, may induce hyperalgesia to cold. In the latter" group, another mechanism may be involved in the development of cold hyperalgesia. Reaction time measurements ${ }^{170}$ and differential nerve block experiments ${ }^{514}$ suggest that in nerve-injured patients myelinated cold-sensitive afferents may also mediate pain. In line with the mechanisms that mediate brush-evoked pain, sensitisation of central pain-signalling neurons may account for abnormal encoding of impulses from non-nociceptive nerve fibres at the llevel of the CNS.

\section{Efferent neural mechanisms}

There are several observations indicating that efferent sympathetic nerve activity in extremities affected by RSD is altered. Firstly, various signs and symptoms of RSD are indicative of autonomic dysfunction. Among these are abnormal temperature (warm or cold) and colour (red or pale) of the skin, as well as altered sweat excretion (hyper- or anhydrosis). Besides, the dystrophic changes that occur have been suggested to be secondary to changes in blood flow to the involved area ${ }^{254}$. The second and more compelling reason for implicating that in $\mathrm{RSD}$ 
patients the activity of the sympathetic nervous system is increased, is the fact that manoeuvres which interfere with efferent sympathetic nerve activity often reduce the patients' pain. For instance, surgical ${ }^{394,425,439}$ or chemical ${ }^{115,235,555}$ sympathectomy as well as pharmacological blockade of $\alpha$-adrenoceptors (phentolamine ${ }^{17}$ and phenoxybenzamine ${ }^{181}$ ) were reported to have beneficial effects on sensory abnormalities in part of RSD patients. Besides, electrical stimulation of sympathetic efferents in nerve-injured patients has been reported to cause pain ${ }^{536}$ In this study, increased discharge rates to adrenalin and to sympathetic trunk stimulation were blocked by the $\alpha$-adrenoceptor blocker phentolamine, but not by the 3 -adrenoceptor blocker propranolol, indicating the involvement of $\alpha$-adrenoceptors. Collectively, these observations have led to the theory that in RSD patients a vicious circle of events is generated which are self-sustaining and which result in an increased rate of efferent sympathetic nerve impulses towards the involved extremity. According to this theory, the initial precipitating event, injury to peripheral tissues or nerves, may lead to alteration in the functioning of primary sensory neurones, either as a direct response to injury or due to changes in milieu in which the afferent terminal lies. The increased afferent activity arising from the damaged periphery is signalled to the spinal cord where it may alter the responsiveness of sympathetic preganglionics as a result of which the rate of efferent sympathetic nerve impulses may become increased (somatosympathetic reflex $)^{57,333}$. This allegedly increased level of efferent sympathetic nerve impulses in combination with the (abnormal) responsiveness of sensory nerve fibres to catecholamines provide an element of positive feedback, thus reinforcing or exacerbating the abnormal sensory inflow. The latter hypothesis seemingly accounts very well for the beneficial effects of sympathectomy in part of RSD patients. However, one should realise that some signs and symptoms of RSD as well as the results of several studies in RSD patients argue against the alleged increase in the rate of efferent sympathetic nerve impulses. Firstly, especially at the early stages of RSD, the skin is often red and warm. This observation, which is indicative of increased skin blood flow, suggests that at this stage efferent sympathetic nerve activity is decreased rather than increased. In line with this phenomenon, in some RSD patients sweat excretion is decreased, which is also suggestive of reduced efferent sympathetic nerve activity. Secondly, microneurographic recordings (in skin fascicles) from sympathetic efferents in RSD patients did not exhibit increased activity ${ }^{71,483,533}$. Thirdly, it has been demonstrated that venous plasma levels of noradrenaline ${ }^{138,218}$ and neuropeptide $Y^{137}$ (co-exists with noradrenaline in sympathetic nerve terminals) collected in the affected limb of RSD patients are decreased when compared with plasma samples obtained from the contralateral (unaffected) extremity.

\section{Skin blood flow}

RSD patients often report an abnormal colour and temperature of the skin of the affected extremity. These abnormalities suggest that skin blood flow in these patients is altered. A noninvasive method to study skin blood flow is laser Doppler flowmetry (LDF). The flow signal recorded with this technique originates predominantly $(>95 \%)$ from the subpapillary arteriolar and venular plexus as well as the interconnecting arterio-venular anastomoses ${ }^{153}$. Several studies have used the LDF technique in RSD patients. Two of these revealed a decrease in skin blood flow at stages II and III ${ }^{441,442}$. Irazuzta ${ }^{247}$ and Cooke ${ }^{93}$ also reported a decrease in baseline skin blood flow in RSD patients, but did not depict the clinical stages. A disadvantage of these LDF studies is that they did not investigate skin blood flow in stage I RSD patients. Moreover, 
these studies failed to investigate the influence of duration of the syndrome on microcirculatory disturbances and generally comprised only a limited number of patients per stage.

Another method to study non-invasively skin blood flow is capillary (video) microscopy. By means of this technique, the superficial capillaries in the dermal papillae, which supply nutrition to the skin, can be directly visualised ${ }^{337}$. In RSD patients, it has been demonstrated by means of this technique that nutritive skin blood flow at stages II and III is reduced ${ }^{441.422}$.

\section{5.d Psychological studies}

There is general agreement that profound behavioural and emotional changes can follow pain in RSD patients. Speculation exists as to whether psychological factors predispose to the development of the RSD syndrome. Most authors have concluded that the behavioural and emotional changes observed in RSD patients are a result rather than a cause of pain $144,355,366$. Few other studies have suggested the possibility of a psychological etiology of RSD ${ }^{147,524}$. A recent metanalysis of 29 papers containing original psychological information of any type finds no evidence for specific psychopathology in RSD patients ${ }^{344}$. Consequently, the author concluded that the specific personality traits noted in RSD are a result of the patients' (often unbearable) ongoing pain and suffering. Besides, in a group of 160 patients with Colles fractures of the wrist van Spaendonck et al ${ }^{479}$ demonstrated that premorbid personality does not differ between those patients who subsequently develop RSD and those who do not. In conclusion, there are no specific premorbid personality traits which predispose to or cause the development of this syndrome. Consequently, in order to prevent misunderstanding, discrediting, ignoring, or even mistreating of the syndrome for a long enough time to permit it to proceed to an irreversible condition, it is of utmost importance to realise that RSD has an organic cause and does not have a psychological etiology.

\subsection{THERAPEUTICAL INTERVENTIONS FOR RSD}

In the past, when no effective treatment was known, RSD frequently resulted in chronic invalidism, mental deterioration, drug addiction, and all too often self destruction. Mitchell reported: "when we first encountered this formidable symptom we exhausted our ingenuity in devices for its relief ${ }^{369}$. Since then, a wide variety of therapies have been recommended for treatment of RSD (table 2.2). The reported rates of success differ considerably. It is generally agreed that an important factor in the effective treatment of RSD is early recognition and treatment since patients with longstanding duration of disease are less likely to respond well ${ }^{403,475}$. Given the wide variety of therapies for RSD, we will discuss only those which are of interest within the scope of this thesis.

The only therapy found to be effective for RSD in large studies is aimed at interruption of the activity of the sympathetic nervous system. Other treatments were found to be successful in isolated reports, but none of them have been proven effective in large studies. Interruption of the sympathetic nervous system activity can be achieved in several ways

Bier block is a technique utilised for regional anaesthesia. The limb is raised and separated from the systemic circulation by a tourniquet. Then substances are injected into the limb intravenously distal to the cuff for a limited period of time after which the tourniquet is 
released. Hannington-Kiff thus injected 10 to $20 \mathrm{mg}$ of guanethidine sulphate, which interferes with transport of noradrenaline while depleting storage in sympathetic nerve terminals. The latter effect is achieved through displacement of noradrenaline from presynaptic vesicles and blockade of its reuptake 187,216. Various authors have reported excellent results for pain relief ${ }^{487.336}$. A disadvantage of this technique is that the duration of pain relief usually last only 12 to 36 hours. This type of treatment is considered to be most effective in patients with hyperpathia and hyperaesthesia as the prominent symptoms ${ }^{187,2155}$. In a series of 47 patients, Loh et al ${ }^{336}$ reported no benefit in $21 \%$, less than 24 hours pain relief in $51 \%$, and pain relief for longer than six months in only $13 \%$ of the cases. In another series of 20 patients in which guanethidine administration was compared with stellate ganglion block, the former proved to be equally effective for pain relief and was slightly longer lasting ${ }^{55}$. Arner ${ }^{17}$ demonstrated that RSD patients responding to guanethidine can be distinguished from non-responders. To this end, (transient) relief of pain following intravenous administration to phentolamine was assessed. Individuals in whom the phentolamine-test was negative did not enjoy pain relief from this type of sym patholysis. Another substance interfering with the storage of noradrenaline in nerve-endings is reserpine. After application of the Bier block, reserpine can be injected intra-arterially as a result of which a gradual depletion of noradrenaline is achieved. In a series of 21 patients, $16(76 \%)$ of those studied responded well to this treatment, although $4(25 \%)$ of these 16 had recurrence of symptoms within a period of 2 weeks to 3 months ${ }^{83}$.

Another method to interfere with efferent sympathetic nerve activity is paravertebral sympathetic ganglion blockade. Many authors consider this treatment the most effective for $\operatorname{RSD}^{114,115,210,235,555}$, although a major disadvantage of this technique is that in part of the patients relief is transient ${ }^{115,475}$. Using this technique, in a series of 69 patients, Steinbrocker ${ }^{485}$ obtained excellent results in $32 \%$, some benefit in $49 \%$, and no benefit in the remaining $19 \%$. Steinbrocker and Argyros, in another series of 26 patients with a follow-up of 3 years, observed excellent results in $32 \%$, good results in $50 \%$, and two recurrences. Shumacker and Abramson $^{475}$, in a series of 32 patients, demonstrated improvement of signs and symptoms in $63 \%$, albeit that the results were permanent in only $33 \%$. Rasmussen and Freedman ${ }^{425}$ reported in a series of 91 patients complete or adequate relief of pain in 50\% of the cases. Prolonged paravertebral sympathetic blockade can be achieved through infusions of local anaesthetics by an indwelling catheter. Using this technique, in a series of 160 patients, Betcher et al ${ }^{41}$ observed an excellent response in $57 \%$ and a good response in $27 \%$. Linson et al ${ }^{331}$, using the same technique, observed in a series of 25 patients with a follow-up of 3 years improvement in $90 \%$ of those studied. However, both studies again were troubled by transient relief of complaints: Betcher et a $^{41}$ observed transient relief predominantly in the more severe cases, whereas Linson et al ${ }^{331}$ reported an overall relapse rate of $25 \%$.

Surgical interruption of efferent sympathetic nerve activity (paravertebral sympathetic ganglionectomy) may be useful in those cases in which relief of complaints following ganglion blocks is only transient. The reported incidence of complete relief of signs and symptoms following this surgical procedure varies from $58 \%$ to $100 \%$ (duration of follow-up in these studies 6 months to 17 years) 3 $34,403,475,560$. Those patients not responding to surgical sympathectomy have been purported to have deficient sympathetic denervation or suffer from severe long-standing disease ${ }^{403,475}$. The results of periarterial sympathectomy are inferior to those of paravertebral ganglionectomy ${ }^{227,425}$. 
Interfering with the activity of the sympathetic nervous system can be achieved not only through intermption of efferent sympathetic nerve impulses but also through blockade of adrenoceptors Ghostine et $\mathrm{al}^{181}$, in a series of 27 patients with $\mathrm{RSD}$ of short duration $(<6$ weeks), investigated the effects of phenoxybenzamine, a non-selective a-adrenoceptor blocker. The drug was administered orally in gradually increasing doses until a maximum daily dose of 40 to $120 \mathrm{mg}$ was reached. Duration of treatment usually was 610 weeks. Duration of followup ranged from 6 months to 6 years. Total resolution was achieved in all cases. Side-effects were minimal and transient, consisting predominantly of mild orthostatic hypotension and ejaculatory problems. In line with these findings, intravenous administration of the nonselective $\alpha$-adrenoceptor blocker phentolamine (for diagnostic and/or prognostic reasons) transiently relieves pain in part of the RSD patients ${ }^{17472}$. Abram and Lightfoot ${ }^{6}$ demonstrated that prazosin, a selective $\alpha_{1}$-adrenoceptor blocker relieves sensory abnormalities in RSD which attests to the involvement of this specific adrenoceptor in the mediation of sensory abnormalities. Scadding et al ${ }^{454}$ investigated the effects of propranolol, a non-selective $B$ adrenoceptor blocker. None of the patients noticed improvement. All together, these data suggest that the $\alpha$-, but not the B-adrenoceptor is involved in mediation of pain in RSD patients.

Rauck et al $^{426}$ interfered with the activity of the sympathetic nervous system through intraspinal administration of the $\alpha_{2}$-adrenergic agonist clonidine. Clonidine but not placebo caused extensive pain relief. The beneficial effects of this technique have been suggested to result from a reduction of efferent sympathetic nerve impulses ${ }^{431}$ and/or decreased transmission. of pain information at the level of the spinal cord ${ }^{563}$.

Stimulation of large myelinated sensory nerve fibres exerts a segmental inhibitory effect on input to certain dorsal horn interneurons ${ }^{21.157}$. Sensory abnormalities in RSD patients have been associated with selective damage of these large myelinated fibres, permitting the balance to favour small fibre activity, thus increasing the transmission of painful afferent impulses ${ }^{361}$. Consequently, sensory abnormalities in RSD may respond to selective stimulation of large myelinated fibres. The latter mechanism may result from transcutaneous nerve stimulation (TNS). A series of eight patients demonstrated that TNS provides long-lasting relief in $25 \%$ of those studied, transient relief in $50 \%$, and no relief in the remaining $25 \%$. In another series of 10 patients, $7(70 \%)$ had complete remission within 2 months, 2 others improved, and one did not respond. Alternatively, dorsal hom interneurons may be activated directly by means of epidural spinal cord stimulation (SCS). Barolat et al ${ }^{29}$ reported a series of 18 RSD patients of which in 15 the SCS system was internalised because of pain relief during a trial period of one week. Follow-up period varied from 4 to 14 months. In the implanted group, pain relief was good in 6, moderate in 5 , minimal in 1 , and absent in 3 . Robaina et al ${ }^{436}$, reported a series of 11 patients of which 8 suffered from RSD whereas the remaining had Raynaud's disease. A good or excellent result was obtained in 10 out of 11 patients (mean follow-up 27 months).

Substance $P$ is released from distal terminals of primary afferent fibres in response to noxious stimuli and may be involved in the inflammatory response associated with cutaneous injury and pain ${ }^{225}$. Besides, it has been demonstrated that certain types of pain sensation are reduced in response to capsaicin which locally deplete substance P. Rumsfield and West ${ }^{449}$, in a series of 33 patients with (postherpetic) neuralgia, studied the effects of local capsaicin application $(0.025 \%)$ to the affected area, four times a day for four weeks. Of the 23 which completed the trial, 18 noted reduction in pain. 


\section{Physical therapy}

- thermal stimulil cold/wet compresses ${ }^{306}$, hot wax applications ${ }^{408}$. warm paraffine package ${ }^{408}$ - ultrasound to the stellate ganglion ${ }^{11}$

- active mobilisation ${ }^{156}$

- passive mobilisation', passive mobilisation under general anesthesia ${ }^{140}$

\section{Drugs applied to the skin}

- $\alpha_{2}$-adrenoceptor agonist clonidine ${ }^{105}$

- local anaesthetic, EMLA cream ${ }^{358}$

- vasodilator; nitroglycerin ${ }^{243}$

- anti-neurogenic inflammation; capsaicin ${ }^{77,450}$

- hydroxyl radical scavengers; dimethylsulfoxide ${ }^{193,194,312}$

Drugs administered regionally (intravenous)

- sympatholytic; guanethidine ${ }^{134,284,351,503}$

reserpine ${ }^{47}$

- analgetic "ketorolac ${ }^{526}$

- anaesthetic; lidocaine $e^{236 ; 515}$

- anti-inflammatory; corticosteroilds ${ }^{410,515}$

- anti-arythmic; bretylium ${ }^{160}$

\section{Drugs administered systemically}

- sympatholytic agents-adrenoceptor antagonist ${ }^{47,18 !}$,

B-adrenoceptor antagonist ${ }^{177,242}$

- antilinflammatory agents; corticosteroids ${ }^{82,295}$, NSAID $^{501,502}$, hydroxyl radical scavengers

(mannitol ${ }^{193}$, N-acetylcysteine ${ }^{197,529}$ )

- hormones; calcitonin ${ }^{349}$

- anti-epileptics: benzodiazepines ${ }^{287}$, carbamazepine ${ }^{423}$, diphenylhydantoin ${ }^{423}$

- (Peripherally acting) vasodillators; calcium reentry blocker (nifedipine) ${ }^{4 / 2}$, serotonin antagonist (ketanserin ${ }^{214,370}$ ), antimycotics (griseofulvin ${ }^{177}$ )

\section{Drugs administered at llevel of spinal cord}

- epidural; steroid ${ }^{125}$, local anaesthetic $c^{262}$, opioid ${ }^{374}$ " $\alpha 2$-adrenoceptor agonist ${ }^{426}$

- intrathecally; opioilds ${ }^{192}$
Nerve blocks with local

anaesthetics

- axillary plexus 228

- interscalene ${ }^{102}$

- suprascapular

- stellate ganglion ${ }^{41,55,331}$

- transient block paravertebral

sympathetic ganglia ${ }^{70,115,235,555}$

- continuous block paravertebral

sympathetic ganglia (indwelling catheter) ${ }^{41,331}$

- pleural ${ }^{432}$

\section{Surgilcal procedures}

- paravertebral sympathetic ganglionectomy $y^{394,425,439,481}$

- peri-arterial sympathectomy ${ }^{227,425}$

Anaesthesiological procedures

- percutaneous thermal

sympatholysis ${ }^{240}$

- radiofrequency lumbar

sympatholysis ${ }^{40}$

\section{Neurosurgical procedures}

- bilateral cingulumotomy ${ }^{452}$

- thalamotomy ${ }^{94}$

- spinothalamic tractotomy $y^{46}$

\section{Electrical neurostimulation}

- epidural spinal cord stimulation ${ }^{29,436 i}$

- transcutaneous nerve stimulation ${ }^{273,437}$

\section{Miscellaneous}

- hypnotherapy ${ }^{\text {t71 }}$

- autogenic training ${ }^{264}$

- thermal biofeedback ${ }^{30}$

- electroconvulsive therapy ${ }^{29: 2}$

- acupuncture ${ }^{421}$

- radiation therapy ${ }^{145}$

- immobilisation with cast or splint ${ }^{155}$

Table 2.2: therapeutical interventions used in RSD patients. 


\section{Chapter 3}

ANIMAL MODELS NERVE INJURY:

GENERAL OVERVIEW 


\subsection{GENERAL INTRODUCTION ON VARIOUS ANIMAL MODELS OF NERVE INJURY}

The two main causes of chronic pain syndromes in humans are complete peripheral denervation and partial peripheral nerve injury in which at least some normal connections of afferent fibres with the periphery are spared. The resulting signs and symptoms of both types of nerve injury as well as their winderlying pathophysiological mechanisms are quite different. Animal models of partial injury of peripheral nerves have appeared only since 1988. Despite their relative recency there is now a considerably body of evidence that these animal models are relevant to human pain conditions such as RSD and causalgia.

\section{1. a Complete transection of a peripheral nerve}

The animal model in which a peripheral nerve is transected completely and the proximal end forms a neuroma ${ }^{541}$ has improved our understanding of the neural mechanisms underlying sensory disorders with complete deafferentiation and neuroma formation ${ }^{117}$. Nevertheless, it represents a type of nerve damage which differs from that in RSD and causalgia patients. Besides, the sensory abnormalities associated with this type of nerve damage do not include all of those that occur in neuropathies in which some of the nerve's normall connections with the periphery are spared (such as hyperalgesia and allodynia).

Nevertheless, this type of nerve injury has provided insight into the pathophysiological mechanisms underlying altered properties of regenerating nerves ${ }^{117}$. Afferents in the midcourse of intact nerves are normally incapable of generating impulses upon slow or prolonged depolarisation. In case of any form of injury or inflammation, Schwann's cells or the axons themselves are damaged which provokes local demyelisation and/or sprout outgrowth. If these regenerating sprouts do not find intact endoneural tubes distally, they are surrounded by an area of tissue injury and inflammatory response. Each axon may sent out multiple sprouts. As a result, a neuroma is formed which is made up of fibrous tissue and trapped fine nerve sprouts. Wall and Gutnick ${ }^{541}$ demonstrated that these sprouts are excessively sensitive to small amounts of catecholamines as well as to mechanical stimuli and may even generate impulses without evident stimulation. The catecholamines which excite the regenerating nerves were suggested to originate from sympathetic nerve fibres growing into the neuroma (along with other nerve fibres) or from the sympathetic innervation of blood vessels invading the regenerating area. In 1983, Devor ${ }^{117}$ summarised the findings of the Jerusalem Pain Research Laboratory which clarified some pathophysiological changes in damaged peripheral nerves. Experiments were performed in experimentally produced neuromas in rats, but also in in-continuity axons that have undergone local demyelisation with lysophosphatidyl choline. Devor proposed that the generation of pain following nerve injury may be related to altered properties of the midaxon part of injured nerves. In non-injured nerves, this part is specialised for impulse propagation and is incapable of generating impulses. However, following nerve section and local demyelisation it becomes also capable of impulse initiation. The spontaneous discharges were suggested to result from tonic depolarisation. Besides, it was proposed that this baseline discharge is changed by stimuli such as mechanical disturbance, elevated extracellular $\mathrm{K}^{+}$, ischemia, and hypercapnia. Adrenergic agonists also proved to be effective depolarizing stimuli: systemically administered adrenaline or noradrenaline usually produced an increase in 
discharge rate. Similar effects were observed following stimulation of preganglionic efferents or the lumbar sympathetic trunk. The site of adrenergic chemosensitivity was considered to be the region of the neuroma itself. It was proposed that this ectopic pacemaker capability relates to abnormal electrogenic membrane properties in the region of demyelisation and sprout outgrowth. These abnormal properties were considered to result from the appearance of excess inward current conductances and ectopic $\alpha$-adrenoceptors. Catecholamines released in the area of nerve injury thus may provoke abnormal afferent discharges.

\section{1.b Partial nerve injury}

Many animal models of partial nerve injury due to trauma, disease, metabolic disorders or toxins have been developed throughout the years ${ }^{14.3}$. Besides, various animal studies have been performed on experimentally induced and naturally occurring chronic compression neuropathies ${ }^{9}$. A common and major disadvantage of these studies is that none of them has been reported to produce disorders of pain sensation like those that accompany partial nerve injury in humans. In 1988, Bennett and Xie ${ }^{40}$ reported on an animal model of partial peripheral nerve injury (chronic constriction injury (CCI)) in which the sciatic nerve was loosely ligated at mid-thigh level. Following this type of (partial) nerve injury, the rats develop not only sensory abnormalities, reminiscent of those observed in RSD (such as hyperalgesia, allodynia, and behaviour suggestive of spontaneous pain), but also other features such as dystrophic claw growth ${ }^{40}$, dystonic foot posture $e^{40}$, and skin temperature abnormalities ${ }^{39,40,533}$ of the affected extremity (table 3.1 , fig. 3.1).

Since that time, various other animal models exhibiting behaviour indicative of hyperalgesia, allodynia, or spontaneous pain have been developed. These models differ from the model of Bennett and Xie, among others, in the site as well as the degree of nerve injury. In the Seltzer model $(1990)^{466}$, about half of the rat's sciatic nerve is ligated high in the thigh. Within a few hours after the operation, and for several months thereafter, the rats develop guarding behaviour of the ipsilateral hind paw which is often licked, suggesting the presence of spontaneous pain. Besides, the plantar surface of the foot was evenly hyperaesthetic to nonnoxious and noxious stimuli. DeLeo et al ${ }^{110}$ observed that freezing of a rat sciatic nerve (sciatic cryoneurolysis) produces characteristics suggestive of neuropathic pain such as autotomy (selfmutilation) which starts at day 4 postinjury and peaks in severity at day 14 . Besides, these authors observed foot edema as well as changes in behaviour indicative of hypaesthesia, touchevoked allodynia, and spontaneous pain. In the Hao model $(1991)^{217}$, rats subjected to photochemically induced spinal cord ischemia, develop behaviours that may reflect tonic and chronic pain as well as responses suggestive of the presence of mechanical allodynia in the dermatomes innervated by the affected spinal segments. In 1992, Kim and Chung ${ }^{280}$ reported on a rat modell in which either the $L_{5}$ spinal nerve alone or both the $L_{5}$ and $L_{6}$ spinal nerve was tightly ligated. In these rats a long-lasting hyperalgesia to noxious heat, mechanical allodynia, and behavioural signs indicative of the presence of spontaneous pain were observed in the affected foot. 


\subsection{THE CHRONIC CONSTRICTION INJURY (CCD MODEL}

Bennet and Xie $\mathrm{X}^{49}$ exposed the common sciatic nerve at the level of the middle of the thigh. Proximal to the sciatic's trifurcation, the nerve is exposed following which 4 ligatures ( 4.0 chromic gut) are tied loosely around it with about $1 \mathrm{~mm}$ spacing. Nerve damage in the $\mathrm{CCI}$ model has been purported to result from chronic compression ${ }^{40}$. This chronic compression may result from intraneural edema which develops subsequent to the initial, partial occlusion of the nerve's vasculature. The force of this edema may oppose the unyielding ligatures as a result of which the nerve self-strangulates. The latter process is identical to the one Sunderland proposes for entrapment neuropathies ${ }^{498}$. Interestingly, Maves et al ${ }^{354}$ demonstrated that chromic gut ligatures laid next to instead of being placed around the sciatic nerve also produced behavioural evidence of neuropathic pain. This finding suggests that not only compression but also a chemical component of the chromic gut sutures may contribute to the injury of the sciatic nerve.

\begin{tabular}{lll}
\hline SIGNS AND SYMPTOMS & RSD & CCI \\
& PATIENTS & RATS \\
\hline
\end{tabular}

Sensory

Stimulus-independent persistent pain

- in affected extremity

- outside distribution of lesioned nerve

- in non-injured (contralateral) extremity

$\begin{array}{ll}313,412,413,528 & .23,296,354 \\ .57,229,4,434,44,4,480 & .283,5065 \\ .294,461,463,464 & .23,280,4,74\end{array}$

\section{Stimulus-induced pain}

- hyperalgesic response to thermal stimuli

- hyperalgesic response to mechanical stimuli

- allodynic response to thermal stimuli

- allodynic response to mechanical stimuli

- slow temporal summation

Autonomic

- abnormal skin colour

- abnormal skin temperature

- edema (clinically)

\begin{tabular}{|c|c|}
\hline $.412,413,149$ & $23,354,346,3$ \\
\hline $.67,71,105,391$ & $.23,283,506$ \\
\hline .71 & $.23,40,208$ \\
\hline $8,71,125,25,4,413$ & $.23,208 i$ \\
\hline$e^{412,413}$ & $.23,40,125$ \\
\hline $.57,287,3113,528$ & .40 \\
\hline . $108,307,327,404,546$ & $.39,40,533$ \\
\hline.$^{3116,395,461,463,551}$ & .40 \\
\hline
\end{tabular}

Trophic

- abnormal growth nail/claw (respectively)

- abnormal consistency nail/claw (respectively)

$.52 \mathrm{~d}$

$n^{125,297,355,528}$

.40

$-29,568,528$
$-40$

Table 3.1: signs and symptoms occurring in RSD patients as well as CCI rats. 


\section{CCI}

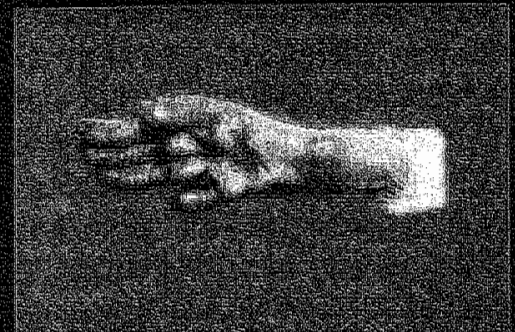

\section{Contral}

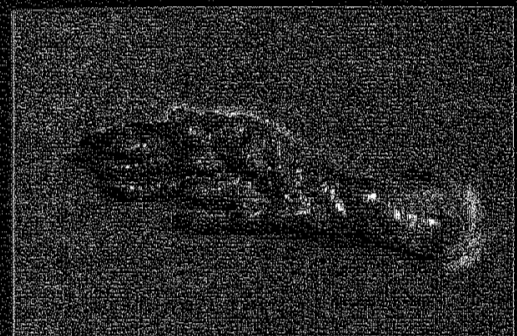

3 days

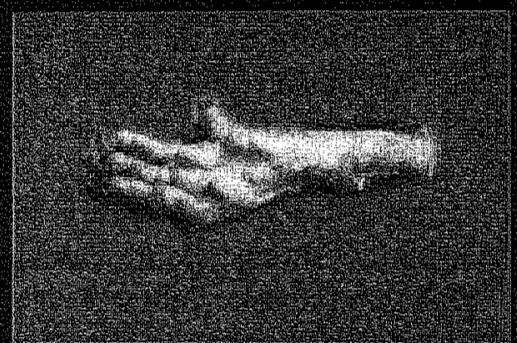

3 weeks

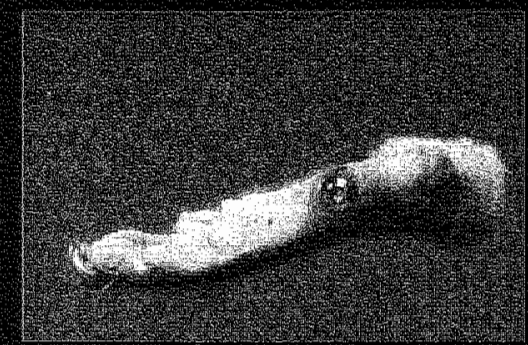

3 months

\section{RSD}

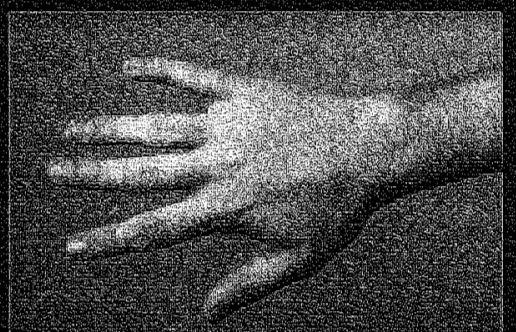

\section{Control}

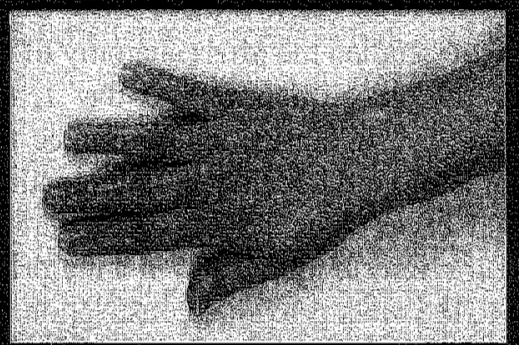

\section{Stage I}

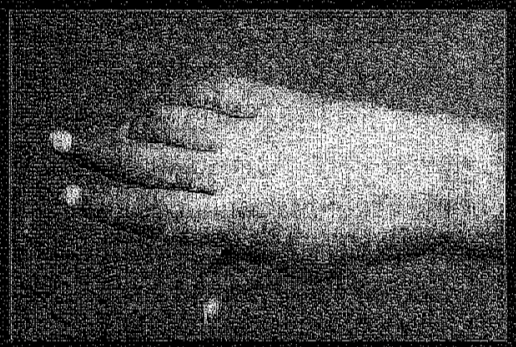

Stage II

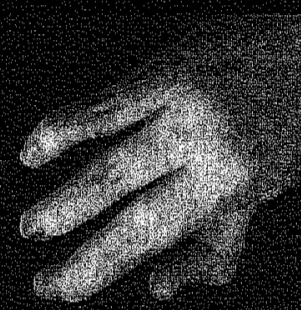

Stage III

Fig. 3.1: similar changes occurring in $\mathrm{CCl}$ rats and $\mathrm{RSD}$ patients. 


\subsection{CHANGES IN THE LOOSELY LIGATED SCIATIC NERVE (CCI MODEL)}

Loose ligation of a sciatic nerve provokes substantial anatomical, morphological, and functional changes. These changes encompass altered efferent and afferent function of fibres in the loosely ligated sciatic nerve. Altered efferent function may account for part of the signs and symptoms observed in $\mathrm{CCI}$ rats. Besides, injury-related discharge from the loosely ligated sciatic nerve towards the centra] nervous system is involved in the development and maintenance of sensory abnormalities. All together, these observations stress the ongoing and central role of the loosely ligated sciatic nerve in the $\mathrm{CCl}$ model and suggest that therapeutical interventions aimed at restoration of normal nerve function may have beneficial effects at an early as well as at later stages after nerve injury.

\section{3.a Gross anatomical changes}

Bennett and $\mathrm{Xie}^{40}$ studied the gross anatomy of the injured sciatic nerve in specimens taken at $1-120$ days after loose ligation. At day 1 , the nerves demonstrate marked constrictions between each of the ligatures, which reduce the nerve's diameter by $25-75 \%$. Epineural vessels were at least partly occluded. Examination of osmium-stained fibres revealed extensive demyelisation. Nerves taken 2-3 days postoperatively demonstrated a progressive tendency for adjoining constrictions to merge and in a majority of the nerves a distinct swelling developed just proximal to the most proximal constriction. Ipsilaterally obtained digital nerves appeared normal at this instant. The ligated regions of the nerve were completely encapsulated in a dense mass of connective tissue that adhered to adjacent muscle. At 1-3 weeks after loose ligation, the nerves demonstrated 2 constrictions or a region of nearly uniform thinning. Nearly all nerves exhibited a pronounced swelling just proximal to the constricted region and most had a distinct but smaller swelling distally as well. The ligatures appeared to lose their continuity 10-14 days postoperatively. At 2-4 months after surgery, the connective tissue capsule had been resorbed. The ligated region remained thinned. Proximal and distal swellings had disappeared. The ligated region appeared opaque, whereas its colour was yellowish. Ipsilaterally obtained digital nerves appeared sometimes thinner than normal, although of normal colour.

\section{3.b Morphological changes}

In various studies light microscopy (LM) and/or electron microscopy (EM) were performed on segments of the loosely ligated sciatic nerves. In general, the results of these studies indicate that loose sciatic nerve ligation causes a partial and differential deafferentiation of the sciatic nerve's territory with survival of primary afferent neurons the axons of which are interrupted. Almost all of the large myelinated $(A B)$ afferents and a preponderance of small thinly myelinated $(A \delta)$ afferents are interrupted at the site of constriction. Consequently, the corresponding axons distal to this site degenerate. Of the small unmyelinated axons (primary sensory afferents and sympathetic efferents), the percentage degeneration distal to the site of constriction differs considerably between various studies (from $20 \%{ }^{376}$ to as much as $84 \%{ }^{69}$ ).

Gautron et al ${ }^{176}$ demonstrated at 2 weeks postinjury a consistent number of fibres smaller than $5 \mu \mathrm{m}$, contrasting with a marked decrease of the large afferent fibres. In addition to an extremely short internodal length, the majority of the fibres had an abnormal myelin sheath. Carlton et $\mathrm{al}^{69}$ showed that at 2 weeks postinjury, nerve segments obtained proximal to the 
lesion appeared nomal at LM level although at EM level occasionally degenerating axons and Schwann cell proliferation were obserwed. The ligated segment itself demonstrated disruption of the perineurium, whereas the connective tissue around each nerve revealed many axons, presumed growth cones, and Schwann cells. The distal segment showed Wallerian degeneration. Some myelinated axons had thin myelin sheaths and were considered regenerating. As compared to the proximal segment, axon counts in the distal segment exhibited significant decrease in both myelinated $(84-99 \%)$ and unmyelinated $(62-84 \%)$ nerve fibres. Besides, a decrease in the proportion of large diameter fibres (larger than $5 \mu \mathrm{m}$ ) was noted in the distal segments. Basbaum et $\mathrm{a}^{31}$ demonstrated at 2 weeks postinjury a near complete loss of large myelinated fibres as well as considerable variation in the damage to small myelinated fibres distal to the nerve Lesion. Additionally, a decrease in the number of unmyelinated axons was observed in the distal segment when compared with the proximal segment. Furthermore, many of the surviving unmyelinated axons in the distal segment were shrunken and distorted, although still in contact with Schwann cells. Munger et al ${ }^{376}$ observed at 10 days postinjury that proximal to the nerve lesion both myelinated and unmyelinated axons are all normal. Nearer to the constriction the authors observed extensive degeneration of myelinated axons and signs of endoneural edema: Distal to the site of ligation, the nerve was uniformly edematous and full of myelinic degeneration. There was a profound loss of large myelinated axons and a distinct less severe loss of small myelinated and unmyelinated axons.

Guilbaud et $\mathrm{a}^{208}$ studied the time course of degeneration and regeneration of myelinated nerve fibres. They observed morphological changes mainly distal to, but also proximal to the ligatures, which is indicative of significant axonopathy with simultaneous degeneration and regeneration. The lesions were at their maximum at 2 weeks after $\mathrm{CCl}$ with progressive recovery beginning between 3-4 weeks. The largest fibres had not totally recovered at 15 weeks.

\section{3.c Functional changes}

When sensory nerve fibres are damaged, a barrage of impulses (injury discharge) is initiated that lasts many seconds or even minutes ${ }^{542}$. Although this injury discharge is only of short duration $^{543}$, it causes a long-lasting, widespread hyperexcitability of the dorsal horn interneurons $^{54,3}$. The presence of this phenomenon in the $\mathrm{CCl}$ model was demonstrated by Dougherty at al ${ }^{126}$. These authors showed that perineural application of a local anaesthetic (lidocaine) before loose ligation induces a reduction in both the duration and magnitude of behavioural hyperalgesia. In line with these findings, in CCI rats Yamamoto et all ${ }^{566}$ demonstrated that application of a local anaesthetic to the sciatic nerve before, but not 15 minutes after, nerve injury significantly delayed the development of hyperaesthesia. In contrast, Mao et al ${ }^{348}$ demonstrated in CCI rats that local anaesthesia of the ligated sciatic nerve through a single perineural bupivacaine injection at day 3 postinjury reduced thermal hyperalgesia when tested 24 hours after injection. All together, these findings indicate that in the CCI model injury-related discharge is involved in the development and possibly also maintenance of sensory abnormalities. Meller et al ${ }^{359}$ investigated in more detail which type of nerve fibre is responsible for the latter phenomenon. These authors compared the development of thermal hyperalgesia between rats treated as neonates with either capsaicin or vehicle. A pronounced thermal hyperalgesia developed in the vehicle-treated but not in the capsaicin-troated group, 
whereas baseline (preligation) witlidrawal latencies to thermal stimuli did not differ between both groups. These findings indicate that the development of thermal hyperalgesia is mediated by capsaicin-sensitive small diameter unmyelinated nerve fibres.

In CCI rats, Kajander and Bennett ${ }^{200}$ recorded the activity of primary afferent axons just before and just after symptom onset. They observed that at day 1 postinjury $85 \%$ of $\mathrm{AB}, 55 \%$ of $A \delta$, and $9 \%$ of $C$-fibres failed to conduct across the injury site. By day 3 , these percentages had increased to $89 \%$ for $A B, 87 \%$ for $A \delta$, and $32 \%$ for $C$-fibres. Spontaneous discharges (usually regular or burning) were observed in $35 \%$ of $\mathrm{AB}, 15 \%$ of $\mathrm{A} \delta$, and $3 \%$ of $\mathrm{C}$-fibres. Of the AB-fibres exhibiting spontaneous discharges, $89 \%$ did not conduct through the injury site; the same was true of $65 \%$ of the Aô-fibres. Both of the 2 spontaneously discharging $\mathrm{C}$-fibres conducted through the injury. Intravenous administration of a $\mathrm{K}^{+}$channel blocker either induced activity in previously silent fibres or increased the frequency of spontaneous activity of $50 \%$ of $A B$ and $19 \%$ of $A \delta$-fibres, whereas no effect was observed on $C$-fibres. Xie and Xiao ${ }^{562}$ observed in $\mathrm{CCI}$ rats changes in electrical activity patterns of damaged fibres alike those previously reported by Kajander and Bennett ${ }^{260}$. These authors additionally demonstrated that various stimuli (such as mechanical pressure, oil drops of $40^{\circ} \mathrm{C}$, electricity) when applied to the injured region of the nerve cause an increase in activity of previously silent fibres and increased frequencies of spontaneously active fibres. Similar responses were observed following stimulation of the $\mathrm{L}_{s}$ sympathetic ganglion and systemic administration of noradrenaline, whereas systemic administration of the $\alpha$-adrenoceptor blocker phentolamine stopped the abnormal firing but did not affect activity in normal fibres. The latter findings attest to the presence of increased catecholamine-sensitivity of sensory nerve fibres in the CCI model. In another study, Kajander et $\mathrm{al}^{2 \cdot 61}$ demonstrated that, at the time of onset of the neuropathic pain syndrome, the spontaneous discharges and sensitivity to $\mathrm{K}^{+}$channel blockade seen in $A B$ and $A \delta$ primary afferents originate in the dorsal root ganglion and not at the injured region of the nerve. Collectively, these data indicate that although the injured nerve's peripheral territory is partially and differentially deafferented, as early as 1 day postinjury the spinal cord dorsal horn is bombarded by high levels of discharge (either spontaneous or stimulus-evoked) from many of the injured nerve's (predominantly myelinated) afferents which have become more sensitive to various stimuli. This phenomenon may contribute to morphological and functional alterations in the CNS and account for some of the sensory abnormalities in the CCI model.

It has been purported that loose ligation of a rat sciatic nerve causes abnormal coupling of sympathetic and sensory nerve fibres which, in turn, may be involved in the development and/or expression of pain-related behaviour. The main reason for believing that this pathophysiological mechanism is present in CCI rats is that administration of the (peripherally acting) sympatholytic drug guanethidine reduces pain-related behaviour in these rats ${ }^{382,407}$ Interestingly, Neil et al ${ }^{832}$ observed that guanethidine, when administered after nerve injury, reduces heat and cold sensitisation considerably, but has less effect on mechanical sensitisation. The authors suggested that this difference in effectiveness may be related to an underlying difference in organisation between sensitisation to either a mechanical or thermal stimulus. In the $\mathrm{CCI}$ model, there is a preferential loss of large myelinated fibres, mostly above the A $\delta$ range $^{176}$. It might be that tactile loss influences the integration of mechanical modalities, relatively more than thermal modalities at the level of the spinal cord. Thus, a central component would be added to the disturbance which, in turn, may be less amenable to 
peripheral treatment. In contrast to the beneficial effects of postinjury administered guanethidine, pre-injury administration increased the severity of self-mutilating behaviour. This differential effect was purported to result from 1) increased levels of circulating noradrenaline after guanethidine-induced depletion of nerve terminals coinciding with the nerve lesion or 2 ) supersensitivity to remaining release of transmitters. Various hypotheses exist as to the origin of the abnormal coupling of the sympathetic and sensory fibres in the injured sciatic nerve. Firstly, it has been purported that following nerve injury structural changes in the nerve allow axons to become very closely associated as a result of which direct flow of current (cross-talk) may develop between them (ephactic connections). However, in experimental studies (in rat and monkey neuromata) few ephapses are seen and only at long postinjury times ${ }^{365}$. Moreover, these ephapses existed only between sensory axons and not between sensory and sympathetic axons. Secondly, it has been suggested that adrenergic sensitivity of injured primary afferent fibres results from an increased number of and/or from more sensitive a-adrenoceptors. The latter phenomenon has been demonstrated extensively in case of several other types of nerve lesions $^{124}$. A third mechanism which may account for abnormal sympatho-sensory interactions in injured peripheral nerves could be that efferent sympathetic activity precipitates a cascade of chemical changes in the micro environment which may lead to production of agents which excite sensory nerve fibres. The latter hypothesis is supported by the finding that effects of adrenergic agents on damaged sensory nerve fibres often have a long latency ${ }^{119}$. One proposed mechanism is that adrenergic agents released from postganglionic neurones activate autoreceptors on the same terminals, stimulating them to release prostaglandins and another byproduct of arachidonic acid metabolism, leukotriene B4. Prostaglandins may then sensitise the terminals of otherwise normal nociceptive afferents thus leading to hyperalgesia. Similarly, leukotriene B4 which can attract and then stimulate polymorphonuclear lakocytes to produce the algogen dihydroxyeicosa-tetraenoic acid (diHETE) which also directly activates nociceptive terminals. A fourth mechanism through which the sympathetic and sensory nervous system may interact is not situated at the site of nerve injury, but in the dorsal root ganglion. McLachlan et $\mathrm{al}^{356}$ diemonstrated in the $\mathrm{CCl}$ model that noradrenergic perivascular axons sprout into dorsal root ganglia and form basket-like structures around large-diameter axotomised sensory neurons. The latter could be activated repetitively by sympathetic stimulation which suggests that an important site of sympatho-sensory interactions and thus of abrormal discharges of sensory nerve fibres in the CCI model is the dorsal root ganglion. The functional importance of the latter mechanism was demonstrated by Kajander et $\mathrm{l}^{261}$ who showed in CCI rats that the spontaneous discharges and sensitivity to $K$ channel blockade seen in $A B$ and $A \delta$ primary afferents originate in the dorsal root ganglion and not at the injured region of the nerve.

The participation of small-diameter afferent fibres in inflammatory reactions has been reported since the end of the last century ${ }^{191,489}$. More recent evidence has suggested that neurogenic inflammation is related to mediator release from unmyelinated sensory nerve terminals that are associated with nociceptors ${ }^{271}$. Substance $P$ (SP) is an 11 amino acid peptide some $80 \%$ of which is synthetised in small dorsal root ganglion cells. It may be transported by peripheral sensory neurones to both peripheral and central endings ${ }^{270}$ and be released on neuronal stimulation ${ }^{318,377}$. Following antidromic stimulation of sensory nerves, neurogenic inflammatory events such as vasodilation, enhancement of vascular permeability, and protein extravasation are observed. It has been purported that SP is an important mediator of such 
events which may result from electrical stimulation, tissue damage, or local axon reflexes. Thus, noxious stimulation of the skin of man leads not only to a flare response (redness of skin), but also to reduced pain thresholds both locally and more widespread ${ }^{326}$. SP exerts its vasodilator effect through direct interaction with post-synaptic receptors (probably of the NK1 subtype) located on endothelial cells of blood vessels ${ }^{15}$ Besides, SP binds to receptors on mast cells, thus causing degranulation and release of inflammatory mediators, such as histamine ${ }^{274}$. Histamine acts indirectly on primary afferents to induce the release of other inflammatory mediators. Besides, it may exert a vasodilator effect. The presence of (SP-mediated) neurogenic inflammation in the CCI model was supported by a study of Basile et al ${ }^{32}$. These authors assessed vascular reactivity via perfusion of SP over the base of a blister raised on the foot pad of CCI rats. A decrease in the vasodilator response to SP was observed $2-5$ weeks postinjury. A bilateral decrease in vasodilation to sodium nitroprusside perfusion in treated rats suggested that part of the altered SP response was due to diminished vascullar reactivity. Besides, plasma extravasation in response to SP was also reduced on the affected side of CCI rats at 4-6 weeks postinjury. The decrease in vasodilator response to SP may result from decreased receptor numbers and/or effectiveness consequent to release of large amounts of SP from sensory nerves through antidromic stimulation. This hypothesis is supported by the observation that in the spinal dorsal horn of CCI rats SP levels are decreased ${ }^{38}$, which may result from increased. release of $\mathrm{SP}$ at peripheral endings of sensory nerve fibres.

\subsection{THE ROLE OF THE SAPHENOUS NERVE (CCI MODEL)}

Abnormal pain sensations that accompany peripheral neuropathies are sometimes found in a distribution that does not coincide with the territories of nerves or posterior roots. This phenomenon has often been used as a line of evidence that patients with neuropathic pain suffer from a psychogenic order. However, this phenomenon may also result from nerve-injury evoked dysfunction of pain-processing neurons in the central nervous system. Kingery et al ${ }^{283}$ demonstrated that loose sciatic nerve ligation reduces withdrawal thresholds to heat and mechanical stimuli not only in the territory of the sciatic but also of the saphenous nerve. Subsequent transection of the saphenous nerves abolished these hyperalgesic responses in the territory of the saphenous but not of the sciatic nerve. In line with these findings, Tall and Bennett ${ }^{506}$ demonstrated that in the CCI model the severity and time course of the mechanoallodynia are similar in sciatic and saphenous nerve territories. Besides, mechano-allodynia in the saphenous territory could be abolished by an acute saphenous transection, but was unaffected by sciatic transection. In contrast mechano-allodynia in the sciatic territory was abolished by acute sciatic transection, but unaffected by saphenous transection. These findings argue in favour of the hypothesis that extra-territorial pain relates to nerve-injury-evoked dysfunction of pain processing neurons in the central nervous system. 


\subsection{CHANGES IN THE SPINAL CORD (CCI MODEL)}

There is considerable evidence that injury of a peripheral nerve provokes substantial anatomical, electrophysiological, and neurochemical changes in the spinal cord ${ }^{37}$. Interestingly, in the CCI model these changes are more alike those produced by nerve transection than those caused by nerve crush ${ }^{38}$. The observed changes suggest that processing of (abnomal) input at this first relay in the pathway of sensory impulses is altened. The latter phenomenon may contribute to abnormal pain sensations seen in nerve-injured rats.

\section{5.a Morphological changes}

Several lines of evidence indicate that high levels of ectopic discharge in damaged primary afferents may be a critical factor for the development of anterograde transsynaptic degeneration of neurons in the superficial laminae of the affected dorsall hom ${ }^{494,495}$. Since loose sciatic nerve ligation has been reported to provoke ectopic discharge in damaged primary afferents, one would expect that this degenerative process is also present in the CCI model. Sugimoto et al ${ }^{493}$ confirmed the latter hypothesis; these authors demonstrated that the lumbar spinal dorsal horns of rats with a chronic ( 8 days) constriction injury contains more degenerated neurons than the cervical dorsal horn. Besides, degeneration was more pronounced in the ipsilateral than in the contra lateral dorsal horn. A second group of CCI rats received a series of 7 daily subconvulsive doses of strychnine (which potentiates transsynaptic degeneration by blockade of inhibitory post-synaptic action). Again, degenerated neutons were observed bilaterally (with ipsilateral predominance), but the incidence was increased 14-fold when compared with rats that did not receive strychnine. The authors hypothesised that the neuronal damage is the consequence of excitatory amino acids (EAA) neurotoxicity, resulting from the barrage of ectopic activity from the injured nerve. Such damage would be enhanced by strychnine and would tend to affect small neurones since they are more susceptible to depolarisation injury. Since these neurones are responsible for pre- and post synaptic inhibition, impairment of their function may reduce afferently-induced inhibition. As a result, the effects of EAA neurotoxicity might be even more pronounced. These findings suggest that a central anatomical abnormality may account for at least some aspects of abnormal pain-related behaviour in CCI rats. In previous investigations degenerative changes in the dorsal horn consequent to inferior alveolar nerve transection invariably required administration of a convulsant $\mathrm{drug}{ }^{495,494}$. It thus appears that the constriction injury promotes transsynaptic cell damage to an unusually high degree.

\section{5.b Functional changes}

A decrease in primary afferent depolarisation (PAD), a mechanism that generates pre-synaptic inhibition $^{448}$, has been described after nerve transection ${ }^{538,539}$. Since in $\mathrm{CCl}$ rats there is axonal degeneration of both myelinated and unmyelinated nerve fibres ${ }^{69}$, one would expect that PAD and thus pre-synaptic inhibition is also impaired in these rats. This consideration prompted Laird and Bennett ${ }^{310}$ to investigate PAD in CCI rats. PAD was quantified by measuring the magnitude of dorsal root potentials (DRPs) evoked in the $\mathrm{L}_{4}$ dorsal root by stimulating the sciatic nerve proximal to the site of injury. The DRP is attributed to the ellectronic spread of depolarisation along primary afferent fibres in the dorsal root and is considered to be a measure of PAD in the population of afferent fibres ${ }^{448}$. Besides, the A-fibre compound action potential 
(CAP) in the nerve proximal to the lesion was measured since the DRP is direetly dependent on the afferent volley. A decrease was observed in the DRP generated by a volley in the injured nerve. This decrease was independent of the decrease in the CAP seen in the injured nerve. These findings suggest that loose sciatic nerve ligation indeed affects the central mechanism responsible for generating primary afferent depolarisation. Thus, the pre-synaptic inhibitory control of the afferent input from the injured nerve is impaired.

Laird and Bennet: ${ }^{305}$ studied ellectrophysiological properties of neurons in the dorsal horn of the spinal cord in CCI rats at the time of peak behavioural abnormalities (days 9-11 postinjury). They observed phenomena, such as loss of peripheral receptive fields (RFs) and loss of low-threshold inputs, which most likely result from the substantial damage to the loosely ligated sciatic nerve. Other phenomena such as responses to stimulation of the injury site, abnormally prolonged responses to stimuli applied to receptive fields, and increased spontaneous activity were considered to be due to central changes and the presence of a novel source of input at the site of nerve injury. The authors suggested that input from the nerveinjury site accounts for sensory abnormalities seen in $\mathrm{CCI}$ rats and may summate with normal input to provoke lowered thresholds to external stimuli. The prominent after discharges seen in some cells were suggested to contribute to abnormally prolonged and intense responses to mild noxious stimuli. All together, these observations suggest that abnormal dorsal: horn mechanisms are involved in abnormal pain-related behaviour seen in $\mathrm{CCI}$ rats.

Palecek et al ${ }^{400}$ studied changes in the properties of dorsal horn projecting neurons in CCI rats. Their study concentrated on the cells of origin of the spinothalamic tract (STT) because this pathway is believed to be one of the most important for conduction of information about painful stimuli from the periphery to higher centres ${ }^{558}$. They observed changes in STT neurons which were most pronounced at days 7 and 14 postinjury. They found that the neurons have high background activity and that the responses to innocuous stimuli represented a larger percentage of the total evoked activity in wide dynamic range neurons. Besides, the occurrence and magnitude of after discharges to mechanical and thermal stimuli were increased. The authors suggested that the increased background activity may relate to the behavioural abnormalities indicative of spontaneous pain, whereas after discharges may relate to prolonged paw withdrawal in response to noxious stimuli which is evidence for hyperalgesia.

There are 2 main reasons for believing that in $\mathrm{CCI}$ rats the sympathetic nervous system is involved in abnormal processing of sensory input at the level of the spinal cord. Firstly, transplantation of adrenal medullary chromaffin cells into subarachnoid space has been demonstrated to reduce allodynic and hyperalgesic responses to thermal stimulations as well as touch-evoked allodynia. This operative procedure appeared to reduce spontaneous painrelated behaviour ${ }^{212}$. Secondly, Kayser et al ${ }^{267}$ demonstrated that the effects of systemically administered clonidine depend on the dosage used. These authors suggested that this differential effect is related to different sites of action of clonidine which might be more likely peripheral for the low doses and additionally central for the high dose. Additional evidence that in nerve-injured rats the sympathetic nervous system is involved in abnormal processing of sensory input at the level of the spinal cord was provided by Ginzburg and Selzer ${ }^{184}$ who demonstrated that adrenal medullary transplants in rats with sciatic nerve transections reduce pain-related behaviour. Besides, Yaksh et al ${ }^{565}$ showed in the Kim and Chung model that intrathecal injection of clonidine reduces mechanical allodynia whereas administration of this 
substance systemically, intraventricularly, or at the level of the cervical spinal cord produces substantially less anti-allodynic action. Various studies have provided data which may shed some light on the mechanisms through which the sympathetic nervous system may modulate processing of sensory input at the level of the spinal cord. In these studies, clonidine applied iontophoretically or topically selectively depressed the responses of dorsal horn neurons to nociceptive inputs ${ }^{163,378,496}$. In the CCI model, various studies have provided data which suggest that the influence of the sympathetic nervous system on processing of sensory input at the level of the spinal cord may be altered. Firstly, it has been demonstrated that the biosynthesis of $\alpha_{2}$ adrenoceptors in the dorsal horn of CCI rats is increased ${ }^{557}$. Secondly, clonidine has been demonstrated to inhibit the release of substance $\mathrm{P}(\mathrm{SP})$, a neuropeptide which supposedly is involved in conduction of nociceptive messages, at the level of the spinal cord. Hence, the reported enhancement of SP binding in the spinal cord of $\mathrm{CCI}$ rats ${ }^{4}$ may also alter the influence of the sympathetic nervous system on processing of sensory input.

\section{5.c Neurochemical changes}

Price et $\mathrm{al}^{414}$ demonstrated that loose sciatic nerve ligation increases glucose utilisation in the dorsal and ventral horns. Peak activity was in laminae V-VI, a region involved in nociceptive processing. The authors suggested that this chronic increase in neural activity in these regions may reflect spontaneous neuropathic pain. Mao et $\mathrm{al}^{\mathbf{3} 47}$ additionally demonstrated that the increase extends from lumbar segment $\mathrm{L}_{1} \mathrm{~L}_{5}$ which may reflect radiation of neuropathic pain.

Substance P (SP) may be transported along dorsal root afferent fibres to terminals located in laminae 1 and 2 in the most dorsal part of the dorsal horn. It has often been suggested that following release from these terminals, SP acts as an excitatory transmitter of primary sensory neurons in the dorsal horn of the spinal cord ${ }^{226,397}$. Nevertheless, discrepancies have been observed in the relationship between release of SP and the response induced, which suggests that SP released in the dorsal horn may possess a trophic function rather than that of a classical, rapidly acting neurotransmitter ${ }^{469,540}$. Studies involving depletion or addition of substance $\mathrm{P}$ in the spinal cord have usually supported a role of SP in nociception. Capsaicin is a neurotoxin which selectively affects unnyelinated (C) and small myelinated (Aס) sensory nerve fibres ${ }^{255}$. When given chronically, it depletes $S P$ in the spinal cord ${ }^{191,379}$. Experimental animals thus treated demonstrate increased pain thresholds to noxious chemical and thermal stimuli $^{251,379}$. Conversely, when SP is injected intrathecally it induces hyperalgesia ${ }^{258,352}$. Barbut et $\mathrm{a}^{28}$ demonstrated that section and ligation but not crush lesion of a rat sciatic nerve produces a depletion of SP in the medial two-thirds of laminae 1 and 2 of segments $\mathrm{L} 4$ and 5 . In the CCI model, it has also been demonstrated that SP immunoreactivity is decreased in the spinal cord ${ }^{169}$, more specifically in laminae I/II and $\mathrm{V}^{38}$. The observed decrease in SP may be related to the damage to small unmyelinated fibres following loose sciatic nerve ligation ${ }^{176}$. However, even a complete sciatic transection kills only a few cells ${ }^{120}$. Alternatively, the decrease in SP may be related to debilitation of SP producing cells, by the $\mathrm{CCI}$, to such an extent that they are incapable of manufacturing much of anything. The finding that sciatic nerve section leads to a greatly increased synthesis of vasoactive intestinal polypeptide argues against this hypothesis ${ }^{468}$. Hence, the decrease in SP most likely relates to selective changes within primary sensory neurons. The latter hypothesis is supported by a study of Nahin et al ${ }^{380}$ who demonstrated that in the CCI model the levels of changes in gene expression within primary 
sensory neurons falls into 1 of 3 categories: those that increase from a moderate basal level of expression (growth-associated protein-43), those that increase from a nearly non-existent level (galanin, neuropeptide $Y$, vasoactive intestinal polypeptide), and those that decrease after the $\mathrm{CCl}$ (calcitonin gene-related peptide and SP). Another mechanism involved in the decrease of $\mathrm{SP}$ in the spinal cord of $\mathrm{CCI}$ rats could be increased release from peripheral endings of sensory nerves through antidromic stimulation. Indirect evidence in support of the latter hypothesis was presented by Basile et al ${ }^{32}$ who demonstrated a decreased vasodilator response to SP in the affected hind paw of CCI rats. The reduced availability of SP in the dorsal horn of CCI rats may account for the increase in SP binding sites ${ }^{172}$.

Excitatory amino acids (EAA) such as glutamate are thought to be neurotransmitters in the mammalian spinal cord ${ }^{549}$. Their actions are being mediated by one of several receptor subtypes which have been classified based on their sensitivities to 3 agonists as $N$-methyl- $D$ aspartate (NMDA), quisqualate (QUIS), and kainic acid (KA) receptors. The latter two are often referred to as non-NMDA receptors due to the similarities in the response following stimulation of either receptor and similarities of sensitivities to agonists ${ }^{103}$. These receptors seem to be associated with different aspects of afferently-evoked excitation; non-NMDA antagonists reduce the monosynaptic excitation in the dorsal horn neuron evoked by primary afferent stimulation whereas NMDA antagonists reduce the polysynaptic excitation in the spinal cord exoked by stimulation of primary afferents ${ }^{104}$. In line with these findings, intrathecally administered EAA agonists produce an increase in caudally directed biting and scratching behaviour whereas intrathecally administration of NMDA (but not QUIS or KA) induces hyperaesthesia. Protracted painful stimulation produces hyperaesthesia and simultaneously causes release of glutamate in the spinal cord ${ }^{437}$. Central facilitation (wind-up) of the activity of dorsal horn wide dynamic range (WDR) neurons consequent to repetitive input from $\mathrm{C}$-fibres ${ }^{362}$ can be markedly attenuated by NMDA antagonists. These data suggest that glutamate acting at the NMDA receptor may play a role in the polysynaptic events which regulate the responsiveness of the spinal systems to $\mathrm{C}$-fibre input. The potential involvement of spinal NMDA- and non-NMDA-receptors in the hyperaesthetic state of CCI rats was studied by Yamamoto and Yaksh ${ }^{567}$. They showed that NMDA- but not non-NMDA-receptor antagonists temporarily eliminate the hyperaesthetic state of $\mathrm{CCI}$ rats at doses below those which altered the response latency of the normal paw or which altered motor function. In another study, Yamamoto et al $^{566}$ demonstrated that intrathecal MK-801. (NMDA-antagonist) when administered 15 minutes before nerve injury delays the development of thermal hyperaesthesia. In contrast, intrathecal administration of MK-801 15 minutes before the nerve injury did not have an effect on the development of thermal hyperaesthesia. These findings suggest that central activation of EAA-receptors plays an important role in development but not maintenance of thermal hyperaesthesia. Sugimoto et al $^{493}$ hypothesised that EAA neurotoxicity is involved in the transsynaptic degeneration in the superficial dorsal horn.

The role of SP and EAAs in CCl-induced sensory abnormalities may involve a common pathway. Skiling et al ${ }^{476}$ demonstrated that intrathecally administered SP causes release of aspartate and glutamate in the cerebral spinal fluid (CSF) of rats with CCI at a dose of SP that did not cause release in sham operated animals. Neonatal capsaicin treatment blocked SPinduced EAA release in both sham and $\mathrm{CCI}$ animals. These findings demonstrate a $\mathrm{CCI}$ induced decrease in the dose of SP required to initiate EAA release in the CSF of the spinal 
cord, a mechanism which might play an important role in CCI-induced sensory abnomalities.

In the spinal cord, endogenous opioid systems have been demonstrated which produce analgesia when activated by exogenous opiates ${ }^{364}$. Spinal opioid receptors are located both preand post-synaptically. Enkephalin peptides are most likely the endogenous ligands for mu and $\delta$ receptors, whereas dynorphin peptides are most likely the endogenous ligands for kappa receptors. It has been demonstrated that in the CCI model enkephalin synthesis is unaltered at days 2,5 , and 10 postinjury whereas a moderate ipsilateral increase is noted at day $20^{1,1}$. Stevens et al ${ }^{487}$ demonstrated a different time course of (bilateral) changes in binding of nu and $\delta$ receptors: $m u$ binding appeared to be increased by day 2 postinjury and returned to control values by day 10 . The decrease in $\delta$ binding developed gradually, with statistically significant decreases appearing at day 10 postinjury. All together these findings suggest that changes in mu and $\delta$ binding are not due to increased activity in enkephalinergic spinal neurons. In contrast, in the CCI model changes in dynorphin synthesis follow a similar time course as changes in kappa binding which suggests that these 2 phenomena are related; dynorphin synthesis is ipsilaterally increased by day 5 postinjury and peaks at day $10^{13}$, whereas binding of kappa receptors decreased rapidly between days 2 and 5 with little change at day $10^{487}$. Al. together, these data indicate that CCI rats have altered responses to opiate analgesics.

Anatomical and neurochemical changes which occur in the spinal cord following loose sciatic nerve ligation may be present not only on the nerve-injured but also on the contra lateral side. Among these are degeneration of neurons in the contralateral lumbar dorsal horns ${ }^{493}$ and increased glucose utilisation in the dorsal and ventral horns ${ }^{347,414}$. These contralateral spinal cord changes may result from commissural connections between intrinsic spinal neurons and/or from primary afferents crossing the midline ${ }^{97}$. Moreover, the presence of these changes contra lateral to the side of nerve injury may be related to the presence of abnormal pain sensation in the contralateral hind paw of CCl rats ${ }^{23,280}$.

\subsection{CHANGES IN THE BRAIN (CCI MODEL)}

Guilbaud et $\mathrm{a}^{206}$ studied changes in neuronal responsiveness in the ventrobasal (VB) thalamic complex of rats 3 weeks after CCI. Their study concentrated on the VB thalamic complex because it receives spinothalamic tract terminals. Besides, it has been shown that changes in neuronal responses of this structure may account for several aspects of hyperalgesia in models of inflammation ${ }^{175,209}$. The authors present evidence of changes in neuronal responsivity in CCI rats, such as higher background activity, fading of the response with repetitive stimulation, increased responses to mechanical stimulation of the affected hind paw, after discharges outlasting the applied stimulus, and abnormal phenomena of sensitisation following application of a non-noxious stimulus. The authors suggested that these abnormalities may be related to the behavioural nociceptive reactions described. Besides, Guilbaud et al ${ }^{207}$ performed recordings in somatosensory neurones of the cortex which participate in aspects of nociception in normal animals. These authors observed in CCl rats dramatic changes in the responses of nociceptive neurones to stimuli applied to the paw with the ligated sciatic nerve and a clear decrease in their activation threshold to mechanical and thermal stimuli. Besides, these findings emphasise that the primary sensory cortex is involved in nociceptive processing. 


\subsection{SIGNS AND SYMPTOMS (CCI MODEL)}

\section{7.a Sensory abnormalities}

Although Kupers et al ${ }^{2 \% 6}$ reported that loose sciatic nerve ligation provokes a neuropathic syndrome in only $30-40 \%$ of the rats, most other authors have demonstrated that this type of nerve injury provokes almost invariably neuropathic signs and symptoms ${ }^{23,40: 533}$. The time course of sensory abnormalities differs between studies performed in various laboratories. In contrast, the time course appeared remarkably constant within the same research group in spite of the fact that the nerve injuries had been inflicted by different investigators ${ }^{16,23,204}$. Various factors may account for the variability in prevalence and time course of sensory abnormalities. Firstly, the degree to which the nerve has to be constricted ("loosely ligated") in order to induce the neuropathic syndrome is not quantitatively defined. Secondly, Seltzer et al ${ }^{466}$ demonstrated in their animal model of neuropathic pain that the sciatic nerve injury should be inflicted at a relatively proximal location (just distal to the branch point of the posterior biceps semitendinosus nerve) in order to obtain an even spread of injured sensory nerve fibres innervating the hind paw. Thirdly, this variability may result from differences in rat strains used; Kupers et $a^{296}$ reported that loose sciatic nerve ligation in Wistar rats provokes neuropathic changes in only a minority of the animals, whereas other authors ${ }^{40}$ have reported neuropathic changes in a large majority of Sprague-Dawley rats subjected to this constriction injury. Fourthly, variability of symptom expression may be related to the use of different quantifiable behavioural approaches. Fifthly, it has been purported that there are important genetic differences in the propensity to which the neuropathic syndrome develops ${ }^{296}$. In fact, it has been demonstrated by Devor and Raber ${ }^{123}$ in their rat model of neuropathic pain (in which a peripheral nerve is completely transected) that autotomy (self-mutilation), which follows this type of nerve injury, is inherited as a single-gene autosomal recessive trait. Finally, variability of symptom expression may be related to differences in age and size of the rats in which nerve injury is inflicted; Tanck et al ${ }^{507}$ observed that larger, older rats fail to develop allodynic responses after loose sciatic nerve ligation. In line with this finding, Chung et al ${ }^{84}$ observed in their animal model of neuropathic pain (tight ligation of L5 and L6 spinal nerves) that young rats displayed much more vigorous behavioural signs of mechanical allodynia and ongoing pain than mature or old rats. All together, these findings attest to the necessity of strictly identical experimental conditions in order to reduce variability of symptom expression.

Various nociceptive tests have been used in studies on neuropathic pain in order to quantify abnormal responses to external stimuli as well as abnormal behaviour indicative of "spontaneous" pain. Allodynic responses to mechanical stimulation can be studied through assessment of 1) the vocalisation threshold to non-noxious pressure using Basile analgesimeter ${ }^{23}$, 2) the withdrawal threshold to non-noxious touch with calibrated von-Frey hairs ${ }^{473}$. Hyperalgesic responses to mechanical stimulation can be investigated through evaluation of the behavioural response to 1) noxious pinch stimuli using a pair of graduated smooth serrated forceps ${ }^{23}$ (hind paw withdrawals, whole limb withdrawals, vocalisation), and 2) pin-prick (sluggish paw withdrawal, quick and robust paw flick, vocalisation + escape + prolonged oral treatment of stimulated paw). Allodynic responses to thermal stimulation can be studied through assessment of the 1) behavioural response (occurrence of struggle) to immersion in a hot $\left(\leq 44^{\circ} \mathrm{C}\right)^{23}$ or cold $\left(10^{\circ} \mathrm{C}\right)^{23}$ water bath, 2$)$ withdrawal thresholds to non- 
noxious heat pulses ${ }^{473}$. Hyperalgesic responses to thermal stimulation can be investigated through assessment of the 1) behavioural response (occurrence of struggle) to immersion in hot water $\left.\left(>44^{\circ} \mathrm{C}\right)^{23}, 2\right)$ withdrawal latencies following exposure to noxious heat pulses ${ }^{473}$, and 3) hind paw withdrawals (number and/or duration) following placement on a $4-5^{\circ} \mathrm{C}$ cold plate $^{40}$. Spontaneous pain-related behaviour can be studied through assessment of the rat behaviour in terms of weighted pain intensity scales with a logical progression of behaviours. These behavioural observations may focus on gait and posture of the affected hind paw ${ }^{25}$ or on general behaviour (eating, drinking, niffing, scratching, etc.) ${ }^{296}$. The degree of automutilation of the affected hind paw can also be used as a measure of "spontaneous, ongoing" pain ${ }^{466}$.

In the $\mathrm{CCl}$ model, extensive investigations have demonstrated behaviour indicative of "spontaneous" pain ${ }^{23,296}$. Besides, it has been demonstrated that this type of nerve injury causes hyperalgesic responses (excessive responses to noxious stimuli) following both thermal ${ }^{23,359}$ and mechanical ${ }^{23,283}$ stimuli. Other studies have demonstrated allodynic responses (nociceptive reaction to usually innocuous stimuli) to thermal ${ }^{40,23}$ and mechanical ${ }^{23}$ stimuli. More rarely, it has been demonstrated that loose ligation of a sciatic nerve causes behaviour indicative of temporal summation (prolonged duration of guarding behaviour after thermal stimulation) ${ }^{23,40}$ and cross-sensitisation (e.g. sensitised responses to mechanical stimulation after non-noxious thermal stimulation $)^{23}$. Interestingly, some studies have demonstrated that loose ligation of a sciatic nerve evokes abnormal pain sensation in the contralateral hind paw ${ }^{23}$.

Although the reported time course of pain-related behaviour in CCI rats differs between various studies, in general these sensory abnormalities develop at about 2-3 days postinjury ${ }^{296}$, are at their maximum at approximately $1-2$ weeks $^{176,23}$, and gradually disappear at about 6-8 weeks ${ }^{23,407}$ (depending on the type of pain-related behaviour assessed). In the study of Attal et $\mathrm{al}^{23}$, it was demonstrated that the time course of the various pain-related disorders (i.e., hyperalgesic and allodynic responses, as well as spontaneous pain-related behaviour) is similar, with a maximum at 2 weeks after ligation and recovery at 2 months. Kupers et al ${ }^{296}$ also investigated the time course of changes in spontaneous and evoked behaviour. These authors observed that scratching, which is considered to be a measure of chronic pain, was increased on the first postoperative day, whereas maximal effects were observed on the 3rd day. The time course of the increased scratching was time-locked with the development of thermal allodynic responses. The walking pattern of the CCI rats normalised after 4 weeks.

Various authors have studied the relation between sensory abnormalities and histological changes in the loosely ligated sciatic nerve. Coggeshall et $\mathrm{al}^{91}$ observed that the onset of hyperalgesic responses to thermal stimuli is accompanied by a preferential loss of large fibres and by a lesser but still substantial loss of small myelinated and unmyelinated axons. The subsequent course of these hyperalgesic responses, however, was not in any obvious way related to the proportions of large myelinated fibres in the affected nerve. Guilbaud et al ${ }^{208}$ focussed on changes in myelinated fibres and related these to allodynic and hyperalgesic responses to thermal and mechanical stimuli. These authors observed the abnormal pain-related. behaviours in these rats appeared to be closely linked to both degenerative and regenerative

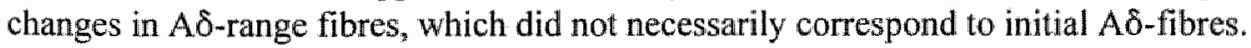




\section{7.b Autonomic (sympathetic) abnormalities}

The important role of the sympathetic nervous system in the CCI model has been demonstrated in various studies. Firstly, loose ligation of a sciatic nerve provokes dystrophic claw-growth (overlong claws with coarse laminations) ${ }^{40}$. This finding may be indicative of autonomic dysfunction, although it can not be excluded that this feature is caused by decreased gnawing.

Another observation which suggests autonomic dysfunction in the $\mathrm{CCl}$ model is the presence of skin temperature abnormalities in the affected hind paw ${ }^{39,40,533}$, which has been demonstrated to evolve from an increase at an early stage to a decrease at later stages ${ }^{39,533}$. Besides, Bennett and Ochoa ${ }^{39}$ demonstrated that transection of the loosely ligated sciatic nerve in the upper thigh produced immediate warming of an abnormally cold hind paw. On the basis of this observation in 2 rats, these authors suggested that this abnormally cold hind paw is due to excessive neurogenic constriction (i.e., increased sympathetic nerve impulses).

Direct evidence of autonomic dysfunction was firstly provided by a study of Wakisaka et $\mathrm{al}^{533}$, who demonstrated that $\mathrm{CCI}$ rats develop a depletion of noradrenaline-containing sympathetic efferents in plantar arteries and veins on the neuropathic side, which could be first noted on postoperative day 5 , was very marked by postoperative days 10-14, and progressed to a complete or nearly complete loss by postoperative day 30 . Besides, these authors observed a concomitant decrease in 2 other substances which are found in sympathetic efferents; dopamine $\mathbb{B}$-hydroxylase and neuropeptide $Y$. Hence, in contrast to the assumption that sympathetic dysfunction in animal models of neuropathic pain consists of increased efferent sympathetic nerve impulses ${ }^{39}$, the findings of Wakisaka et al ${ }^{533}$ suggest that the level of activity of the sympathetic nervous system is reduced.

\section{7.c Motor abnormalities}

Several studies have demonstrated that loose ligation of a sciatic nerve provokes an abnormal gait as well as an abnormal posture of the hind paw ${ }^{23,466}$. Besides, atrophic changes of muscles in the affected hind paw have been observed ${ }^{40}$. It is most likely that these abnormalities are at least partly related to the sensory abnormalities which cause guarding behaviour and thus disuse. However, Filliatreau et al ${ }^{160}$ provided evidence that the chronic constriction injury also damages the motor neuron. These authors compared changes in axonal transport of acetylcholinesterase (AChE) between sciatic nerves subjected to either loose ligation or crush injury. They observed a rapid initial decrease in AChE transport in the CCI model which, however, was delayed by 24 hours when compared to the crush injury model. Hence, the loose ligatures do not immediately injure the nerve axons but rather induce a secondary process. At day 3 postinjury, the $\mathrm{AChE}$ transport was decreased by $60 \%$ in the $\mathrm{CCL}$ model and by $82 \%$ after crush which indicates that more axons were spared in loosely ligated nerves. In the CCI model, the $\mathrm{AChE}$ transport recovered rapidly during the 3 rd week postsurgery indicating that most of the injured neurons were reconnecting their target cells. The authors additionally compared the time-course of nociceptive manifestations and changes in AChE transport. In the $\mathrm{CCI}$ model, $\mathrm{AChE}$ transport was already impaired several days before the first nociceptive manifestations. In contrast, $\mathrm{AChE}$ transport normalised more rapidly than nociceptive behaviour. These differences in time-course demonstrate that changes in gait and posture of the hind paw do not relate solely to sensory abnornalities but involve additionally damage to the motor neuron. 


\subsection{THERAPEUTICAL INTERVENTIONS (CCI MODEL)}

Various studies have focussed on the effectiveness of therapeutical interventions which modulate the activity of the sympathetic nervous system, either peripherally or at the level of the central nervous system. In the $\mathrm{CCl}$ model, it has been demonstrated repeatedly that systemic administration of the (peripherally acting) sympatholytic drug guanethidine has an antinociceptive effect ${ }^{382,407}$. Guanethidine is a rather specific blocker of postganglionic sympathetic nerve function; initially it releases stored noradrenaline and thereafter blocks further release. On repeated dosing, tissue stores of noradrenaline are almost depleted ${ }^{3}{ }^{3} 4$. Neil et al ${ }^{382}$ treated $\mathrm{CCI}$ rats systemically' with guanethidine for 4 consecutive days commencing 5 days before or 10 days after ligation. Behavioural parameters were followed for 4 weeks after drug treatment. Heat and cold sensitisation were reduced considerably, although less effects were observed on mechanical sensitisation. If administered before surgery, guanethidine rather increased the severity of self-mutilating behaviour. In line with these findings, Perrot et al $\mathrm{a}^{407}$ demonstrated that systemic administration of guanethidine in $\mathrm{CCl}$ rats at 1 week postinjury reliably reduced and even suppressed the abnormal reactions to cold stimulus. Modulation of activity of the sympathetic nervous system at the level of the spinal cord has also been demonstrated to have an antinociceptive effect in CCI rats. Firstly, adrenal medullary transplants in $\mathrm{CCl}$ rats reduce pain-related behaviour ${ }^{212}$. Secondly, Kayser et a ${ }^{267}$ demonstrated that systemic administration of the $\alpha_{2}$-adrenoceptor agonist clonidine at 2-3 weeks postinjury only has a moderate effect on the abnormal reactions to mechanical stimuli but dramatically increases the struggle latency to thermal stimuli. The latter effects could be completely prevented by prior administration of the $\alpha_{2}$-adrenoceptor antagonist idazoxan. In other models of partial nerve injury, it has also been demonstrated that interrupting efferent sympathetic nerve activity (through systemic guanethidine administration ${ }^{474,281}$ or surgical resection of sympathetic chain from $L_{2}$ to $L_{6}$ levels ${ }^{279,281}$ ) reduces pain-related behaviour. In line with these findings, systemic administration of the competitive non-selective $\alpha$-adrenoceptor antagonist phentolamine reversibly relieved mechanical allodynia ${ }^{281}$. Besides, alike observations in the $\mathrm{CCl}$ model, it has been demonstrated in other models of partial nerve injury that modulation of activity of the sympathetic nervous system at the level of the central nervous system may influence pain-related behaviour. Administration of $\alpha_{2}$ (clonidine) but not of $\alpha_{1}$-adrenoceptor agonists provokes a dose-dependent antinociceptive effect ${ }^{267,565}$. Clonidine delivered systemically, intraventricularly, or at the level of the cervical spinal cord produced less antiallodynic action, which suggests that this $\alpha_{2}$-adrenoceptor agonist reduces preganglionic sympathetic outflow at the level of spinal preganglionic neurons.

As alluded to before, it has been demonstrated in $\mathrm{CCl}$ rats that the NMDA receptor is involved in the hyperaesthetic state of CCI rats ${ }^{567}$. This involvement may account for the potent antinociceptive affects in CCI rats of MK-801 (Dizocilpine maleate, MW $=221$, Merck Sharp and Dohme Research Lab), a non-competitive NMDA-receptor antagonist. Beneficial effects were observed, in case of both intrathecal ${ }^{566,567}$ and systemic ${ }^{101}$ administration, on thermal hyper algesia ${ }^{101,348}$, thermal hyperaesthesia ${ }^{566,567}$, as well as spontaneous nociceptive behaviour ${ }^{348}$. There is general agreement that MK- 801 , when administered before nerve injury, prevents the development of nociceptive behaviour ${ }^{101,566}$. In contrast, conflicting evidence exists as to the role of MK-801 in the treatment of pain-related behaviour. Yamamoto et al ${ }^{566}$ reported that 
MK-801 when administered 15 minutes after nerve injury does not have an effect on the development of nociceptive behaviour. Others have reported a (temporary) reduction of nociceptive behaviour in case of postinjury administration of MK-801 ${ }^{348}$.

The adequacy of opioid substances in alleviating neuropathic pain is highly con troversial $^{18,159}$. One should bear in mind that the assumption that neuropathic pain is insensitive to opioids is often based on anecdotal observations. In contrast with this classical view, it has been demonstrated that in the CCI model at 2 weeks postinjury intravenously administered morphine reduces tonic pain-related behaviour (indicative of spontaneous pain) ${ }^{257}$. At this instant, naloxone elicits a bidirectional dose-dependent action: increased pain-related behaviour with the high dose and reduced pain-related behaviour with the low dose ${ }^{257}$. This bidirectional dose-dependent effect was demonstrated to be related to the intensity and duration of hyperalgesia ${ }^{24}$. Attal et $\mathrm{al}^{22}$ demonstrated that systemic morphine also has dose-dependent naloxone-reversible antinociceptive effects on phasic painrelated behaviour in CCI rats. Interestingly, morphine seems to have a potent antinociceptive effect with tests of pain evoked by mechanical stimuli but seems unable to alleviate thermal allodynic or hyperalgesic responses ${ }^{316}$. This differential effect of opioids on nociceptive tests based on different stimulus modalities and intensities may be due to activation of different types of fibres and/or to the progressive increase in the number of activated fibres in response to different intensities of stimuli. The antinociceptive effects of morphine appears to be significantly more potent on the ligated side of $\mathrm{CCI}$ rats than those observed in unoperated rats, which suggests that this type of nerve injury increases morphine's analgesic potency ${ }^{22,257}$. Kayser et al ${ }^{268}$ demonstrated in $\mathrm{CCI}$ rats that the augmented analgesic potency of morphine may partly result from a peripheral action of this substance, as indicated by a dose-dependent reduction of the effect of morphine following intra plantar injection of naloxone. In contrast to polyarthritic rats ${ }^{269}$, in CCI rats morphine toleranee does not seem to develop abnormally rapid ${ }^{75,383}$.

Ardid and Guilbaud ${ }^{16}$ evaluated the effect of tricyclic antidepressant drugs on neuropathic pain related behaviour at 2 weeks after $\mathrm{CCI}$, the time of the maximum hyperalgesia. They observed that acutely injected clomipramine, desipramine, and amitriptyline increase (naloxon-reversible) the vocallisation-threshold to paw pressure, whereas amitriptyline additionally reduced spontaneous pain-related behaviour. When chronically injected, the drugs induced a progressive increase in vocalisation threshold with a time-course parallel to that of suspected plasma levels. The analgesic action of tricyclic antidepressants may relate to the monoaminergic spectrum of the drug in relation to the opiate systems.

Systemic administration of lidocaine has been shown to produce analgesia in a variety of neuropathic pain states ${ }^{25,54}$. This effect may be related to an action at the injured site of the peripheral nerve or by an effect on sensory processing in the dorsal horn of the spinal cord. In $\mathrm{CCI}$ rats Abram and $\mathrm{Yaksh}^{7}$ demonstrated that systemically administered lidocaine reduces behavioural responses to noxious thermal stimulation by two distinct mechanisms. Blockade of nociceptor-induced spinal sensitisation was achieved incompletely and only at blood levels that are close to those associated with symptoms of toxicity. Besides, no effects were observed on previously established spinal hypersensitivity. These findings suggest that the predominant effect of systemic lidocaine is through suppression of spontaneous impulse generation arising from injured nerve segments or associated dorsal root ganglia. 


\section{Chapter 4}

MATERIALS AND METHODS 


\subsection{REFLEX SYMPATHETIC DYSTROPHY}

\section{1.a Patients}

All RSD patients met the standards of the American Association for Hand Surgery on the definition of RSD: diffuse pain, loss of function, and objective evidence of significant autonomic dysfunction ${ }^{12}$. Additionally, the signs and symptoms had to be present in an area Jarger than and distal to the area of primary injury or operation. Autonomic dysfunction had to be reflected by an abnormal temperature sensation and at least one of the six following symptoms: edema, increased sweating, abnormal hair or nail growth, and atrophy of skin or subcutaneous tissues. In a minority of cases, RSD patients experience a cold sensation in the affected extremity immediately after the onset of the condition. Since the pathophysiology of this so-called primary cold RSD might be of a different origin than that of primary warm RSD $^{528}$, we restricted ourselves to the latter. The skin areas in which the microcirculation were examined had to be within the area affected by RSD, with respect to both pain complaints and sensation of abnormal skin temperature. Patients (and controls) receiving cardiovascular medication, suffering from diabetes mellitus or with a history of sympathectomy were excluded from the studies since these factors may influence (micro) circulatory parameters.

The large number of and variability in signs and symptoms which may be present in RSD patients attests to the necessity of categorisation of these signs and symptoms. In our experimental studies in RSD patients, we slightly modified the classification systems used by Schwartzman ${ }^{460,462}$ and Jänig ${ }^{253}$. Signs and symptoms were divided into 4 categories: (1) autonomic disturbances (abnormal temperature sensation, edema, increased edema on dependency, abnormal nail growth, abnormal hair growth, and hyperhydrosis), (2) trophic disturbances (cutaneous atrophy, subcutaneous atrophy, and abnormal consistency nails), (3) motor disturbances (loss of function, reduced strength, muscle cramps, muscle wasting, tremor, dyscoordination, and involuntary movements), (4) sensory disturbances (diffuse pain, increase pain during exercise, hyperalgesia, mechanical allodynia, warmth intolerance, cold intolerance, hypaesthesia, and paraesthesia. One should realise that this categorisation system is used solely for the purpose of clarification; signs and symptoms may be related to various underlying pathophysiological mechanisms. For instance, loss of function, reduced strength, and dyscoordination may be related to both motor and sensory abnormalities. Hence, the categorisation system used does not reflect undisputed causal relations.

The considerable overlap in signs and symptoms between the three stages makes it virtually impossible to classify the patients unequivocally with the traditionally used classification systems ${ }^{60,350}$. For the purpose of more reproducible categorisation, in our studies we confined ourselves to the patients' perception of skin temperature of the clinically affected hand as compared to the clinically unaffected hand. Moreover, categorisation on the basis of the patients' perception of skin temperature may take into account the actual regulatory state at the time of investigation, which is of importance because in RSD patients skin temperature (and thus skin blood flow) has been reported to demonstrate short-term fluctuations ${ }^{58}$. Thus, before the start of the experimental protocoll patients with a stationary warmth sensation were categorised stage I, patients with an intermittent warmth and cold sensation were categorised stage II, and in case of a stationary cold sensation, patients were categorised stage III. 


\section{1.b Methods}

All experimental protocols had been approved by the ethical committee of our institution. All measurements were executed following a period of 20 minutes of acclimatisation in a room with temperature between $24^{\circ} \mathrm{C}$ and $25^{\circ} \mathrm{C}$. Measurements were pertormed in the clinically affected and/or clinically unaffected upper extremities of RSD patients as well as in upper extremities of age- and gender-matched controls. All subjects had given informed consent in advance of the experimental procedures, refrained from smoking, and did not consume caffeine or alcohol during at least one hour before investigation.

\section{Microcirculatory investigative techniques}

\section{Laser Doppler flowmetry}

Total (mainly thermoregulatory) skin blood flow was measured non-invasively by means of a laser Doppler flowmetry (LDF) instrument which has been described in detail before ${ }^{387}$. This instrument generates laser light which passes through a flexible fibre-optic towards the measuring probe. In human skin, the penetration depth of the laser light contributing to the signal is about $0.6 \mathrm{~mm}^{530}$, whereas the measured volume is approximately one $\mathrm{mm}^{3}$. In the skin, the emitted light is backscattered by the tissues and blood cells moving in superficial vessels (fig 4.1). The laser Doppler flow signal is derived from the Doppler shift of the reflected laser light. The value thus obtained is directly proportional to microcirculatory blood flux, i.e., the product of the number and mean velocity of the moving blood cells.

Measurements were executed with the Perimed PF3 (Perimed, Linköping, Sweden), containing a low power $2 \mathrm{~mW}$ Helium-Neon laser source which produces monochromatic laser light with a wave length of $632.8 \mathrm{~nm}$. During the experiments, the following instrument settings were employed: time constant of 2 seconds, in order to avoid heart beat oscillations in the signals; low pass band filter "on", in order to reduce movement artifacts; frequency limit $0.07-12 \mathrm{kHz}$ band, unless perfusion was so low that switching to the $4 \mathrm{kHz}$ band was required. The output was digitised by an analog to digital converter and subsequently saved on hard disk. Off-line analysis was performed, employing a software programme which was developed in our institute. Biological zero flow was subtracted from all LDF values measured. The laser Doppler probe holder can be set at any level between $26^{\circ} \mathrm{C}$ to $44^{\circ} \mathrm{C}$, or can be switched off. The heating element was either switched off of set at $40^{\circ} \mathrm{C}$. The LDF instrument was calibrated on 250 perfusion units (equal to 2.5 Volts), using the Periflux Motility Standard.

In RSD patients, LDF was performed in the most symptomatic finger of the clinically affected hand and in the corresponding finger of the clinically unaffected hand. In controls, measurements were performed in the $4^{\text {th }}$ digit of the left hand. In all (seated) subjects, the laser Doppler probe holder was attached to the pulp of the finger with double sided adhesive tape. To avoid rotation of the probe, it was fastened to the holder with adhesive tape. The pulp of the finger was chosen because the richly sympathetically innervated arteriovenous anastomoses are found solely in the skin of the palmar side of the hands ${ }^{478}$. The flow signal obtained from this specific skin area mainly $\left(>95 \%{ }^{153}\right.$ ) originates from the (neurally-controlled) thermoregulatory part of the skin microcirculation (i.e., venous plexus and arteriovenous anastomoses) and only to a minor extent from the (non-neurally controlled) nutritive part (i.e., superficial capillaries). 


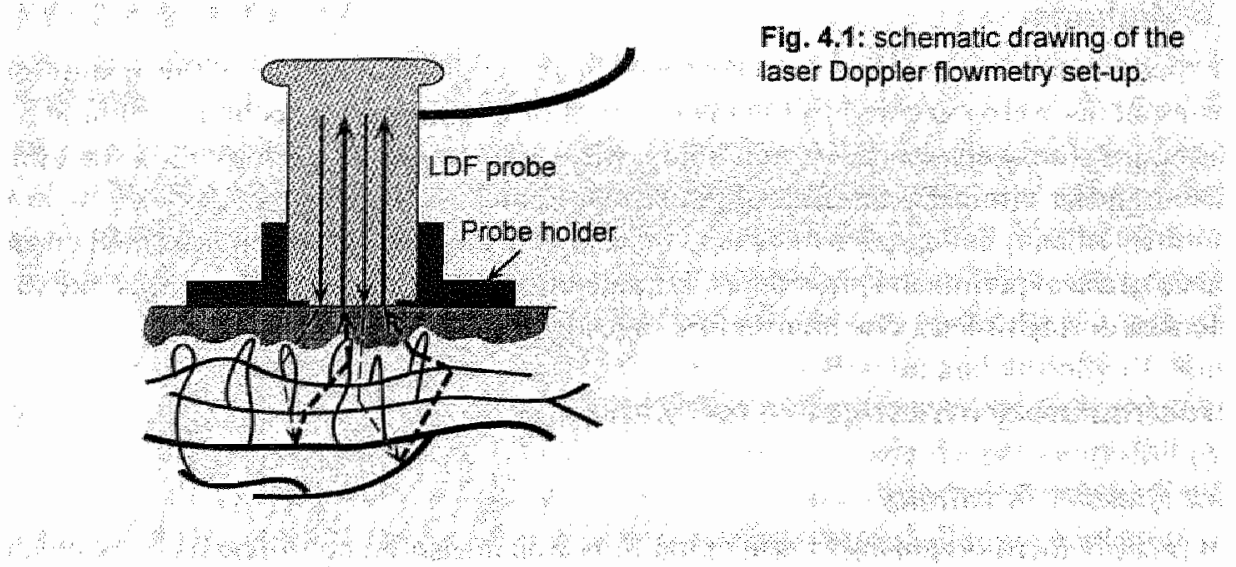

LDF was initially performed, under resting conditions, with the hand at heart level. Thus, an impression was obtained about (mainly thermoregulatory) skin blood flow under physiological conditions.

In part of the investigated subjects, we additionally assessed changes in the LDF signal resulting from several provocation tests. Firstly, we assessed LDF at ambient temperature during reactive hyperaemia, following release of three minutes arterial occlusion. To obtain arterial occlusion we inflated a cuff placed around the upper arm to $230 \mathrm{~mm} \mathrm{Hg}$. After acute deflation of the cuff we assessed peak flow ( 3 minutes recording). Subsequently, the individual peak flow-to-rest flow ratios, which were defined as the flows obtained at rest divided by those obtained during reactive hyperaemia, were calculated. It has been demonstrated previously that the coefficient of variability of this parameter is lower than that of skin blood flow under resting conditions ${ }^{422}$. Besides, this parameter provides an impression about the possible role of larger vessels between the upperarm and hand on microvascular disturbances ${ }^{51,521}$ and constitutes a relative measure for the vasodilatory capacity of skin microvessels ${ }^{422}$. Secondly, after an interval of at least 15 minutes we assessed changes in the LDF signal resulting from incomplete suppression of local vasoconstriction. To this end, the laser Doppler probe holder was heated to $40^{\circ} \mathrm{C}$. Subsequently, the LDF signal was again allowed to equilibrate until a stable signal was obtained for at least 3 minutes following which flow was recorded, both under resting conditions ( 3 minutes recording) and during postocclusive reactive hyperaemia ( 3 minutes recording). This provocation test also provides an impression about the possible rolle of larger vessels on microvascular disturbances ${ }^{519,521}$ and about the vasodilatory capacity of skin microvessel $\mathrm{s}^{422}$. Thirdly, we assessed changes in the LDF signal resulting from a change in posture of the investigated limb. To this end, after an interval of at least 15 minutes, the arm was lowered until the fingers were $35 \mathrm{~cm}$ below heart level. Subsequently, the LDF signal was again allowed to equilibrate until a stable signal was obtained for at least 3 minutes following which flow was recorded, both under resting conditions ( 3 minutes recording) and during postocclusive reactive hyperaemia ( 3 minutes recording). The posturally induced change in skin blood flow which is observed at the thermoregulatory level of the skin microcirculation is predominantly controlled by a sympathetically mediated veno-arteriolar response of local 
The reproducibility of LDF has been documented earlier and, hence, was not repeated here. Lukkari et al ${ }^{341}$ showed that the mean coefficient of variation (CV) for LDF is 0.11 in case of 15 minutes intervals (without detachment of the probe) and 0.30 from day-to-day. Tenland et $\mathrm{al}^{509}$ showed that, with the probe in the same position, the CV for LDF on forehead and forearm skin is between 0.04 and 0.19 in case of 20 minutes intervals and 0.25 in case of one hour intervals. These authors additionally showed that the intra-individual spatial differences for LDF in forearm skin areas is $0.1 \%$.

\section{Skin temperature}

Skin temperature was measured non-invasively. Measurements were executed using an electronic thermometer ( $78214 \mathrm{C}$, Hewlett Packard) attached to a circular metal thermocouple $\left(0.8 \mathrm{~cm}^{2}\right)$. The output was digitised by an analog to digital converter and subsequently saved on hard disk. Off-line analysis was performed, employing a software programme which was developed in our institute. In RSD patients, assessment of skin temperature was performed in the most symptomatic finger of the clinically affected hand and in the corresponding finger of the clinically unaffected hand. In controls, measurements were performed in the $4^{\text {th }}$ digit of the left hand. In all (seated) subjects, the metal thermocouple was fixed to the skin of the dorsum of the finger by means of adhesive tape. After attachment of the thermocouple skin temperature was measured, under resting conditions, with the hand at heart level. Thus, an impression was obtained about skin temperature under physiological conditions. In part of the investigated subjects, we additionally assessed skin temperature with the hand in the dependent position.

\section{Transcutaneous oximetry}

The tissue pressure of oxygen that diffuses from skin blood vessels to the skin surface was measured non-invasively by means of a transcutaneous oximetry $\left(\mathrm{TC} p_{\mathrm{O}}\right)$ instrument, which has been described in detail previously ${ }^{87}$. Briefly, this technique measures, at the skin surface, the tissue pressure of oxygen that diffuses from skin blood vessels. The transcutaneously measured tissue ${ }_{p O 2}$ is derived from the electrical potential changes caused by a redox reaction that takes place in the electrode between a platinum cathode and a silver anode in the presence of oxygen. The resulting current is a measure of the concentration of oxygen present.

Measurements were executed with the Radiometer TCM2 transcutaneous oximeter (Copenhagen, Sweden), containing a membrane-covered Clark-type electrode ${ }^{87}$. This electrode contains a heating element which can be set at various levels between $37^{\circ} \mathrm{C}$ to $45^{\circ} \mathrm{C}$. In our experiments, the thermistor-controlled heating element was set at either $37^{\circ} \mathrm{C}$ or $44^{\circ} \mathrm{C}$. Subsequently, the system was calibrated to room air (atmospheric oxygen tension) ${ }^{24 !}$. TC $p_{02}$ measurements can not be performed at local (unheated) skin temperature because the transcutaneously measured tissue $\mathrm{pO}_{2}$ values in non-hyperaemic adult skin are nil or close to zero. Local skin heating causes dilatation of superficial blood vessels as well as a shift of the oxygen dissociation curve to the right ${ }^{241}$. This causes release of oxygen from oxyhaemoglobin and thus, a higher blood $p_{\mathrm{O} 2}$ for the same total oxygen content. The increase in blood $p_{\mathrm{O} 2}$ is estimated to be approximately $6 \%$ per ${ }^{\circ} \mathrm{C}$. Then, oxygen diffuses from the skin vessels in the proximity of the epidermal basement membrane towards the surrounding tissues. Depending on the rate of blood flow, the $p_{\mathrm{O} 2}$ reduction resulting from this transit is about $30 \mathrm{~mm} \mathrm{Hg}$, whereas the average mid-capillary $p_{02}$ amounts to approximately $125 \mathrm{~mm} \mathrm{Hg}$. Nevertheless, 
the $p_{02}$ drops in the passage from the superficial vessels to the surface of the skin as a result of the presence of metabolically highly active cells in the epidermis and the keratin layers of the outer epidermis. Besides, a minor portion of the remaining oxygen is consumed by the electrode itself In normal skin, the $\mathrm{TC} p_{\mathrm{O}}$ on the heated surface has been demonstrated to be $33 \mathrm{~mm} \mathrm{Hg}$ lower than the midcapillary $p_{\mathrm{O} 2}$. The output of the TCM2 transcutaneous oximeter was digitised by an analog to digital converter and subsequently saved on hard disk. Off-line analysis was performed, employing a software programme which was developed in our institute.

In RSD patients, TCp $p_{02}$ was performed at the dorsum of the hand in the intermetacarpal space immediately adjacent to most symptomatic finger of the clinically affected hand and in the corresponding skin area of the clinically unaffected hand. In healthy volunteers, measurements were performed in the intermetacarpal space immediately adjacent to the $4^{\text {th }}$ digit of the left hand. In all (seated) subjects, the skin area to be investigated was cleaned with alcohol in order to remove any barriers which may interfere with oxygen diffusion. Then, the TC $\mathrm{PO}_{2}$ measuring electrode was attached to an intact part of the skin by an adhesive fixation ring containing contact liquid. After attachment of the measuring electrode, the signal was allowed to equilibrate until a stable signal was obtained for at least 3 minutes.

TC $p_{\mathrm{O}_{2}}$ was initially performed under resting conditions with the hand at heart level and at a probe temperature of $37^{\circ} \mathrm{C}$. At this probe temperature, the $T C p_{02}$ signal reflects the tissue $p_{\mathrm{O} 2}$ during incomplete suppression of local vasoconstrictive mechanisms ${ }^{521}$ and depends on arteriolar flow rather than arteriolar $p_{\mathrm{O} 2}$.

In part of the investigated subjects, we additionally assessed changes in the $\mathrm{TC}_{p 02}$ signal resulting from several provocation tests. Firstly, we assessed $T C p_{02}$ during reactive hyperaemia, following release of three minutes arterial occlusion. To obtain arterial occlusion, we inflated a cuff which was placed around the upper arm to $230 \mathrm{~mm} \mathrm{Hg}$. After acute deflation of the cuff we assessed peak flow ( 3 minutes recording). This provocation test was performed in order to get an impression about the possible role of larger vessels between the upperarm and hand on microvascular disturbances ${ }^{521,519}$ as well as to obtain a relative measure for the vasodilatory capacity of skin microvessels. Secondly, after an interval of at least 15 minutes we assessed changes in the $T \mathrm{~T}_{\mathrm{O}_{\mathrm{O}}}$ signal resulting from complete suppression of local vasoconstriction. To this end, the $\mathrm{TC} p_{\mathrm{O} 2}$ probe was set at $44^{\circ} \mathrm{C}$. At $44^{\circ} \mathrm{C}$, $\mathrm{TC} p_{\mathrm{O}_{2}}$ values depend only on the arteriolar $p_{\mathrm{O} 2}$ and are not influenced by microcirculatory flow ${ }^{87}$. After this change in probe temperature, the $T C p_{02}$ signal was again allowed to equilibrate until a stable signal was obtained for at least 3 minutes. Then, the signal was recorded, both under resting conditions ( 3 minutes recording) and during postocclusive reactive hyperaemia ( 3 minutes recording). This provocation test also provides an impression about 1) the possible role of larger vessels on microvascular disturbances ${ }^{521,519}$ and 2 ) the vasodilatory capacity of skin microvessels.

The reproducibility of transcutaneous oximetry has been previously documented in extenso and, hence, was not repeated here. Lukkari et al ${ }^{341}$ showed, in patients with severe occlusive arterial disease, that the mean coefficient of variation (CV) for TC $p_{02}$ is 0.19 in case of 15 minutes intervals (without detachment of the probe) and 0.37 from day-to-day. If TC $p_{\mathrm{O} 2}$ values below $10 \mathrm{~mm} \mathrm{Hg}$ were excluded the corresponding CV were 0.08 and 0.31 . 


\section{Intravital video capillary microscopy}

The (non-neurally controlled) nutritive part of the skin microcirculation was assessed noninvasively by means of a the intravital video capillary microscopy technique (fig. 4.2 ), which has been described in detail before ${ }^{248}$. This technique enables visualisation of superficial skin capillaries. In most areas of the human skin, the capillary loops run perpendicular to the skin surface as a result of which only the apex of these loops can be visualised. Such areas are the most appropriate to measure capillary density. In contrast, in the nailfold area of hands and feet the capillary loops run parallel to the skin surface. The terminal rows of capillaries can usually be visualised in their full length, and are therefore the most appropriate ones to investigate both capillary morphology and capillary perfusion.

Visualisation of nailfold capillaries was performed with a Leitz Ploemopak 2.1(1.25 X) system and a Leitz POL (Polarizer)-cube for incident illumination. The light from a $100 \mathrm{~W}$ mercury arc was projected directly through the POL-cube and the objective lens onto the nailfold. We used mercury light because its spectrum has intensity peaks at wavelengths of about 400 and $430 \mathrm{~nm}$. At these wavelengths, most of the light is absorbed by haemoglobin which enhances the contrast between red blood cells and the surrounding tissues. Neutral density filters in the illuminating light path were sometimes used to adjust the light intensity to the camera. We used a Leitz $4 x$ objective (numerical aperture (NA) 0.14 ) to assess capillary density, whereas a Leitz 10x objective (NA 0.30 ) was used to assess capillary diameter and red blood cell velocity. The POL-cube consists of a 50\% transmission / $50 \%$ reflection mirror in a $45^{\circ}$ position with respect to the optical axis of the microscope. The POL-cube accommodates a polarizer in the illumination pathway and a crossed analyser in the image forming pathway to cancell light reflections from lenses and skin surface ${ }^{61}$. A heat absorption/reflection filter was installed in the illumination pathway to prevent a rise in the temperature of the nailfold skin. Magnification at the front plane of the TV camera (Philips Newvicon XQ 1275, 2/3 inch tube), which was positioned in the intermediate plane of the microscope, was $5 \mathrm{x}$ (Leitz $4 \mathrm{x}$ objective) or $12.5 \mathrm{x}$ (Leitz 10x objective). For assessment of capillary diameter and red blood cell velocity, the TV camera was rotated in order to orientate the capillaries parallel to the video lines. Images were displayed on a monitor screen (Philips LDH $2122 ; 12$ inch) with a final magnification of $165 \mathrm{x}$ (Leitz $4 \mathrm{x}$ objective) or $410 \mathrm{x}$ (Leitz 10x objective). Simultaneously, images were stored on videotape through a videocassette recorder (Sony Betarnax SL-C9 ES) for off-line analysis, using either a temporal correlation method incorporated in the automated Capiflow software ${ }^{415}$ or a flying spot device ${ }^{517}$. The Capiflow software produces two photometric windows which were positioned over the arteriolar limb of the capillary. Blood cells and plasma gaps passing through the capillary modulate the light intensity in the two windows. These fluctuations in the upstream window, often with obviously recognisable peaks and throughs, appear in the downstream window with some delay. The delay equals the time needed for the red blood cells to travel the distance between the 2 windows. The time-delay between the 2 signals was assessed through cross-correlation of these signals. The velocity is considered to be the ratio of the preset distance between the 2 windows and the time-delay. The flying spot device generates dots which move over the monitor screen with a known (adjustable) speed. Red blood cell velocity can be assessed through synchronisation of moving dots with the blood cells and plasma gaps in the arteriolar branch of the capillary. Besides, this technique allows assessment of capillary diameters: the spots can be positioned halfway 
between the visible base of the arteriolar limb and the crest of the loop. Subsequently, the spot width is adjusted until it exactly overlaps the capillary width. The distance between this tmeasuring site and the capillary crest ranged from about 50 to $250 \mu \mathrm{m}$. Within this distance, the capillary diameter did not vary significantly. Data analyses were performed by a single investigator, thus avoiding inter-observer variability. Calibration was performed by taping a fim scale and assessing the conversion factor to real scale.

In RSD patients, capillary microscopy was performed in the nailfold of the most symptomatic finger of the clinically affected hand and of the corresponding finger of the clinically unaffected hand. In healthy volunteers, measurements were performed in the nailfold of the $4^{\text {th }}$ digit of the left hand. In all (seated) subjects, the hand to be investigated was placed on the stage of the microscope. Relative motion between the skin and the microscope was prevented by embedding the finger in a mass of clay. The nailfold to be investigated was covered with a drop of paraffin oil in order to reduce skin reflections and improve skin transparency. Capillary density was assessed in one video screen about $1.6 \mathrm{~mm}$ (width of one sereen) proximal to the terminal row of capillaries, which has an effective surface area of 1.86 $\mathrm{mm}^{2}$. In this specific skin area, the capillaries run perpendicular to the skin. Subsequently, capillary diameter and capillary red blood cell velocity (RBCV) were assessed in usually 4 , but at least 3 capillaries located in the terminal rows of the nailfold area.

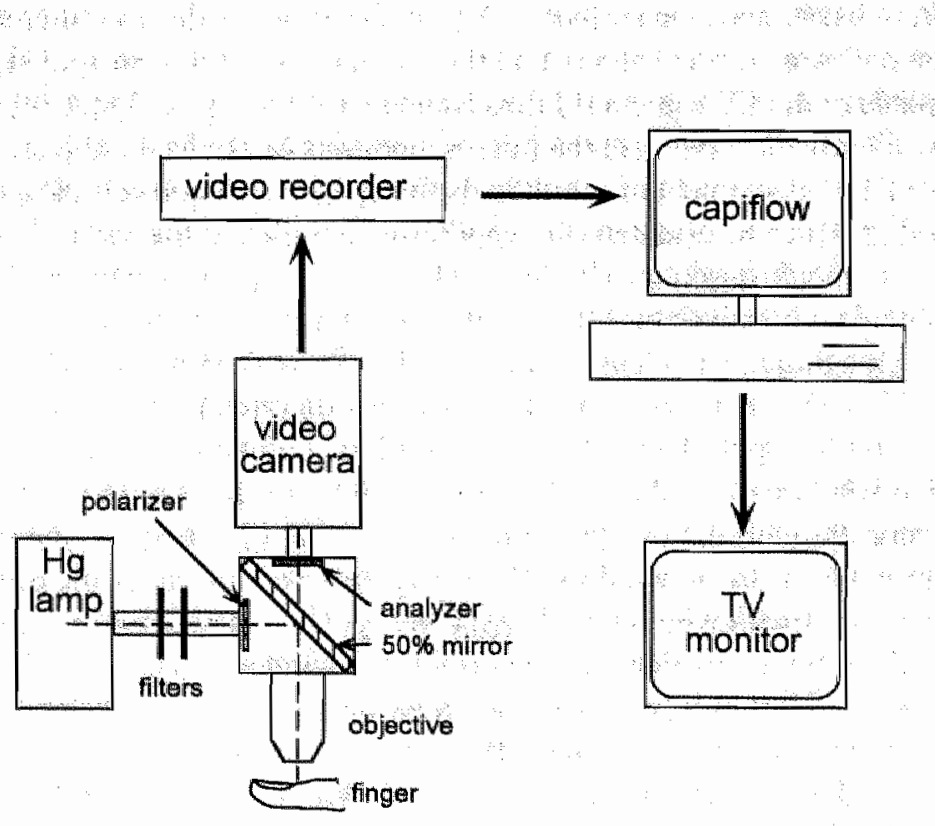

Fig. 4.2: schematic drawing of the capillany microscopy set-up (in humans) 
RBCV was initially assessed under resting conditions with the hand at heart level. Thus, an impression was obtained about nutritive skim blood flow under plyysiological conditions.

In part of the investigated subjects, we additionally assessed changes in RBCV resulting from several provocation tests. Firstly, we assessed RBCV during reactive hyperaemia following release of one minute arterial occlusion. To obtain arterial occlusion, we inflated a cuff which was placed around the upper arm to $230 \mathrm{~mm} \mathrm{Hg}$. After acute deflation of the cuff we assessed peak flow (one minute recording). This provocation test was performed in order to get an impression about the possible role of larger vessels between the upperarm and hand on microvascular disturbances ${ }^{519}$. Secondly, we assessed changes in $\mathrm{RBCV}$ resulting from a change in posture of the investigated limb. To this end, the measurements at heart level were followed by a 15 minutes period during which the stage of the microscope was lowered $35 \mathrm{~cm}$ and the patient was allowed to adapt to the dependent position. Subsequently, measurements of RBCV (both under resting conditions and during postocclusive reactive hyperaemia) were repeated. The posturally induced changes at the nutritive level of the skin microcirculation are considered to be controlled mainly by (non-neural) local factors such as tissue oxygenation and vasoactive metabolic substances accumulating during ischemia ${ }^{519,547}$. Hence, sympathetic dysfunction is likely to disturb to a greater extent vasomotor responses to dependency at the predominantly neurally controlled thermoregulatory level of the skin microcirculation than those at the non-neurally controlled nutritive level.

The reproducibility of capillary microscopy has been documented earlier ${ }^{518}$ and, hence, was not repeated here: the (intra-observer, intrasubject, intersession) coefficient of variation (CV) for the red blood cell velocity under resting conditions was $59 \%$, whereas the $\mathrm{CV}$ for the maximum red blood cell velocity during reactive hyperaemia was $25 \%$.

\section{Macrocirculatory investigative techniques}

\section{Vessel wall Doppler tracking}

The uitrasonic vessel wall Doppler tracking (VWDT) system was developed by Hoeks et al ${ }^{230}$. Briefly, the system consists of a conventional B-mode echo imaging system (Ultramark IV; ATL, Bothell, Washington, USA) and a data acquisition system connected to a personal computer. With a $7.5 \mathrm{MHz}$ transducer, a two dimensional $\mathrm{B}$-mode image is created from the vessel of interest. An M-line perpendicular to the vessel is selected. After the echo system is switched to M-mode, storage of data starts. During 5-6 cardiac cycles radiofrequency (RF) signals are digitised and temporarily stored in an 1 Mbyte memory. The $\mathbb{R}$-top of the simultaneously recorded electrocardiogram (ECG) was used as a trigger to maintain a constant time-relationship between the distension measurement of the vessel and the ECG. The first RJ" line acquired is used to identify the wall lumen interfaces. "The position of the anterior and posterior vessel walls are marked by the observer using two data windows on the first RF signal stored. Line after line the data are transferred to a personal computer. The distance between both sample volumes corresponds to the initial diameter of the vessel lumen (minimum diameter at end diastole). The cumulative change in phase between the successive RF lines is calculated for the anterior and posterior wall windows, where the position of the sample gates is continuously adjusted according to the detected displacement. After processing all lines, the displacement waveform of the arterial walls is displayed on the sereen (fig. 4.3 ). 


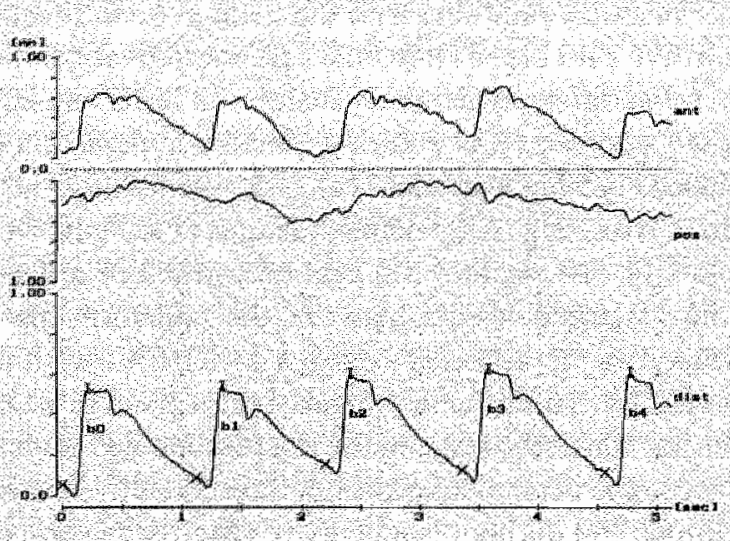

Fig. 4.3 . displacement waveforms of the anterior (top tracing) and posterior (middle tracing) wall of the brachial antery This results in the distension waveform bottom tracing), where a cross indicates the occurrence of an ECG-trigger.

The distension waveform, i.e., the change in diameter as a function of time is obtained by subtracting the posterior displacement waveform from the anterior waveform (difference in displacement between the anterior and posterior wall). The above procedure is an off-line analysis and provides data on arterial diastolic diameter (D) and distension $(\triangle \mathrm{D})$ for each captured beat. From $\Delta D$ and $D$, the relative distension $(R D, \triangle D / D)$ of the brachial artery was calculated. RD is the fractional diameter increase (and thus represents the local strain of the arterial wall). An advantage of this approach is that a change in mean position of the artery caused by probe movements and/or changes in vessel position will not really influence the outcome of the measurement.

In RSD patients, VWDT was performed of the brachial artery in the clinically affected upper extremity. In healthy volunteers, VWDT measurements were performed in the right upper extremity. Simultaneously, digital arterial blood pressures were assessed in the second finger of the ipsilateral hand. In all (supine) subjects, during the measurements the echo transducer of the VWDT was constantly kept in place in order to avoid changes in measured values caused by differences in distension along the arterial tree ${ }^{433}$. The brachial artery was chosen because in the investigated patient group the events initiating RSD were localised at the level of the wrist. Hence, VWDT allows assessment of sympathetic (dys-) function proximal to the site of the alleged nerve injury. If autonomic dysfinction in RSD results from damage to sympathetic axons at the site of the precipitating trauma, a discongruity in sympathetic dysfunction may be expected between sites proximal to and distal to this trauma.

VWDT was initially performed under resting conditions. Thus, an impression was obtained about diameter and distension of the brachial artery under physiological conditions. In part of the investigated subjects, we additionally assessed changes in these parameters resulting from the cold pressor test (CPT). To this end, the contralateral clinically non-affected hand was emerged, to the level of the wrist, into water at $0^{\circ} \mathrm{C}$ for 1 minute. This CPT evokes sympathetic stimulation ${ }^{438}$. It has been demonstrated in humans that the consequent hemodynamic responses are mediated by stimulation of sympathetic nerves rather than stimulation of adrenal glands ${ }^{319}$. Hence, the change in relative distension of the brachial artery induced by the CPT may be used as a measure of sympathetic innervation of this artery. 
The reproducibility of VWDT has been documented earlier and, hence, was not repeated here. Kool et al ${ }^{292}$ showed that the CV for detemination of the diameter of the brachial artery is 0.03 (intra-observer, intrasession) and 0.03 (intra-observer, intersession). The CV for distension was found to be 0.13 (intra-observer, intrasession) and 0.13 (intramobserver, intersession).

\section{Finapres}

Arterial blood pressure in the finger was measured non-invasively by means of a Finapres device ${ }^{245}$. The Finapres technique has been described in detail previously ${ }^{554}$. Briefly, the blood volume under an inflatable cuff is assessed with an infrared plethysmograph and kept constant to a set-point value by adjusting culf pressure in response to volume changes in the tinger artery. At zero transmural pressure, intra-arterial finger pressure matches cuff pressure. Cuff pressure is then recorded. The operation of the apparatus is fully automated. It has been demonstrated that by means of this technique similar information can be obtained as with invasive recording of blood pressure ${ }^{245}$. Besides, this technique not only allows continuous assessment of blood pressure under resting conditions but also assessment of blood pressure responses to orthostatic stress ${ }^{245}$.

Measurements were executed with a commercially available Finapres device (Ohmeda. 2300 Finapres NIBP; Ohmeda, Eaglewood, Colorado, USA). This device contains a built-in expert system (Physiocal) which establishes a proper volume clamp set point. This set point is automatically adjusted at regular intervals. To this end, blood pressure recording was interrupted for $2-3$ beats every 70 beats. Regular Physiocals are necessary to keep the finger arteries completely unloaded and the transmural pressure equal to zero ${ }^{554}$. The output (systolic, diastolic, and mean blood pressure as well as pulse rate) was saved on hard disk. Off-line analysis was performed, employing a software programme which was developed in our institute.

In RSD patients, digital arterial blood pressure (DABP) was measured in the second most symptomatic finger of the clinically affected hand and in the corresponding finger of the clinically unaffected hand. If only one finger was symptomatic, DABP was measured in the most symptomatic finger following the assessment of skin microcirculation. In healthy volunteers, measurements were performed in the $3^{\text {th }}$ digit of the left hand. In all (seated) subjects, the (best fitting) cuff of the Finapres device was wrapped around the middle phalanx of the finger to be investigated.

Assessment of DABP was initially performed under resting conditions with the hand at heart level. Thus, an impression was obtained about DABP under physiological conditions. In those subjects in which vessel wall characteristics of the brachial artery were studied by means of VWDT, we additionally assessed changes in DABP following contralateral application of the cold pressor test (described in detail under heading VWDT).

The reproducibility of the finapres technique has been documented earlier ${ }^{232}$ and, hence, was not repeated there: the (intra-observer, intrasubject, intrasession) coefficient of variation (CV) for the pulse pressure under resting conditions was $3.7 \%$.

\section{In vitro investigation of subcutaneous arteries}

Functional ${ }^{402}$ aspects of sympathetic innervation as well as sensitivity to noradrenaline ${ }^{386}$ can be investigated adequately in subcutaneous arteries. This prompted us to study these aspects 
in arteries that had been isolated from subcutaneous fat of amputated extremities of 2 RSD patients. Experimental data from the neuropathic side were compared with those from arteries isolated from subcutaneous fat of the contralateral extremity. Both patients had given informed consent in advance to the operative procedure and met previously reported standards of RSD. The areas of the amputated limbs from which the subcutaneous arteries were obtained were affected by RSD, with respect to both sensory abnormalities and perception of abnormal skin temperature. All experiments were performed according to procedures described in detail later in this chapter (4.2.2).

Briefly, in order to examine mechanical properties, a segment of the isolated arteries was mounted on two stainless-steel wires with a diameter of $40 \mu \mathrm{m}$ and placed horizontally in a myograph between an isometric force transducer and a displacement device for recording of isometric force development ${ }^{107,375}$. The organ chamber was filled with Krebs-Ringer bicarbonate solution, which was maintained at $37^{\circ} \mathrm{C}$ and gassed with $95 \% \mathrm{O}_{2} / 5 \% \mathrm{CO}_{2}$. Before experimentation, all arteries were distended to their individual optimal lumen diameter, by stepwise increases in diameter with intermittent exposure to 5-HT. Subsequent experimentation was performed at this optimal diameter.

Firstly, contractile responses to electrical field stimulation (EFS) were evaluated as a functional measure of sympathetic innervation. To this end, two platinum electrodes were placed along the longitudinal axis of the isolated vessel preparations. These electrodes were connected to a constant current source delivering electrical impulses of constant duration at a variable frequency. These conditions of electrical field stimulation (EFS) have been reported to selectively stimulate intramural adrenergic nerves in isolated blood vessels.

Secondly, the contractile responses to cumulative doses of the non-selective adrenoceptor agonist noradrenaline were assessed as a measure of the sensitivity to this catecholamine. Concentration-response curves were obtained by increasing the concentrations in half-log increments. The ratio of the maximal response to EFS and the maximal response to noradrenaline was calculated as a functional measure of adrenergic innervation.

\section{1.c Methodological considerations}

Various experimental factors necessitated supplementary methodological measures.

In subjects in which several microcirculatory investigative techniques were combined, laser Doppler flowmetry, transcutaneous oximetry, and assessment of skin temperature were performed simultaneously whereas capillary microscopy was performed in advance of the other techniques. We opted for this sequence instead of random allocation because the duration of arterial occlusion was shorter for capillary microscopy than for the other techniques. Besides, capillary microscopy and the other techniques were performed with an interval of at least 30 minutes. In nearly all capillary microscopy experiments, reactive hyperaemic responses had subsided within one to two minutes, as has also been demonstrated by others ${ }^{518}$. Hence, it is unlikely that the induction of reactive hyperaemia during capillary microscopy has an effect on the results of the other techniques.

In those subjects in which microcirculatory measurements at heart level were followed by the same measurements in the dependent position, we opted for a time-interval of at least thirty minutes in order to allow adaptation to the dependent position as well as to ensure that the previous hyperaemic response had subsided. A similar time-interval was used in those 
subjects in which microcirculatory parameters were assessed at various levels of suppression of local vasoconstrictive mechanisms (i.e., at various levels of local skin heating).

In those subjects in which microcirculatory responses to dependency as well as changes in vessel wall characteristics of the brachial artery following cooling of the contralateral hand were assessed, we intially performed LDF and subsequently VWDT. We opted for this sequence instead of randon allocation because the induction of the cold pressor test induces systemic effects, whereas the microcirculatory response to dependency is of local origin.

Since reflex sympathetic dystrophy patients frequently suffer from allodynia and hyperalgesia, they usually could not tolerate the inflated cuff for longer than one minute without moving their hand under the microscope. We, therefore, opted for the induction of one minute arterial occlusion in the case of assessment of the hyperamic response during capillary microscopy. Since movement of the hand does not interfere with laser Doppler flowmetry, we opted for the induction of three minutes arterial occlusion in the case of assessment of total (mainly thermoregulatory) skin blood flow. In our opinion, this difference is allowed, because arterial occlusions of one minute ${ }^{520}$ and three minutes ${ }^{521}$ have been shown to induce a clear-eut hyperaemic response.

\section{1.d Statistics}

Unless stated otherwise, the following statistical methods were used. Patients characteristics are presented as means with ranges (age, duration of syndrome) or as prevalences (signs and symptoms, smokers, events precipitating RSD). Hemodynamic data are presented per group (controls or clinical RSD stage) and as a function of duration of the syndrome. Since the data obtained were not equally distributed, group walues were characterised by medians, together with their interquartile ranges, below which fall $75 \%$ and $25 \%$ of the data, respectively. The non-parametric Mann Whitney-U test was used to test for (interindividual) differences between the three patient-groups as well as between a specific patient group and controls. For the analysis of intra-individual differences (e.g., between pre- and post-provocation values or between the clinically affected and clinically unaffected hand) the paired Wilcoxon signedranks test was used. Differences were regarded statistically significant when $\mathrm{P}<0.05$. We used linear regression analysis to describe the microcirculatory values as a function of the duration of the syndrome in order to study the influence of time on potential microcirculatory disturbances. Regression equations ( $\mathrm{Y}=$ constant $-\mathrm{A}$ (slope) * duration of disease (months)) are presented with $95 \%$ Confidence Intervals $(\mathrm{Cl})$ of the slope and the correlation coefficients (R). Since the duration of complaints is displayed on the X-axis on a logarithmic scale, the linear regression line is represented by a curve. 


\subsection{CHRONIC CONSTRICTION INJURY}

\section{2.a $\mathrm{CCl}$ rats}

All studies were carried out under a protocol approved by the Institutional Animal Care Committee of the Maastricht University, The Netherlands. Male Lewis rats (local inbred, Maastricht University, The Netherlands) were used weighing 250-350 grams at the start of the experiment. The rats were housed individually in standard laboratory cages. They received food (type RMM-TM, Hope Farms, Woerden, The Netherlands) and water ad libitum. The animals were divided into 2 groups; a first group which was subjected to loose ligation of the right sciatic nerve and a second unoperated group.

As alluded to in chapter 3 , several experimental animal models of partial nerve injury are presently under active study. We opted for the Bennett and Xie model for various reasons. Firstly, this model not only reproduces behavioural pain manifestations but also other features observed in RSD such as dystrophic claw growth ${ }^{40}$, dystonic foot posture ${ }^{40}$, and skin temperature abnormalities ${ }^{39,40,533}$ of the affected extremity. Secondly, in this model the precipitating nerve-injury is situated in an extremity which, at least in surgical practice, is mostly also the case in RSD patients. Thirdly, the constrictive procedure applied in the CCI mode ${ }^{40}$ may reflect better neuro-anatomical abnormalities in RSD patients than constriction of only part of a peripheral nerve ${ }^{466}$. We realise that in part of the RSD patients the precipitating injury is not situated at the level of large (mixed) peripheral nerves (such as the sciatic nerve) but at the level of the central nervous system or at the level of peripheral nerve twigs. Nevertheless, signs and symptoms caused by nerve injury at these different sites may be mediated by comparable pathophysiological mechanisms. The observation that injury to a large mixed peripheral nerve causes also degeneration of terminal nerve twigs distal to the site of injury ${ }^{499}$ argues in favour of this hypothesis. An additional argument in favour of the hypothesis that signs and symptoms caused by nerve injury at different sites may be mediated by comparable pathophysiological mechanisms is that signs and symptoms in rats with partial injury of a large (mixed) peripheral nerve are alike those observed in RSD patients without established injury of a large (mixed) peripheral nerve.

The chronic constriction injury of the rat sciatic nerve was induced as follows. Firstly, the animals were anaesthetised with $100 \mathrm{mg} / \mathrm{kg}$ ketamine hydrochloride i.p. (Nimatek, AUV, Cuijk, The Netherlands) and $0.5 \mathrm{mg}$ diazepam s.c. (Centrafarm BV, Etten-Leur, The Netherlands). The administration of ketamine hydrochloride was repeated ( $30 \mathrm{mg} / \mathrm{kg}$ i.p.) if necessary. The operative procedure was performed under aseptic conditions. With the help of a dissecting microscope ( $40 \mathrm{x}$ magnification), about $7 \mathrm{~mm}$ of the right sciatic nerve was exposed from high-thigh to mid-thigh level, by blunt dissection through the biceps femoris, just proximal to the trifurcation into the tibial, sural, and peroneal nerves. Subsequently, the sciatic nerve was loosely ligated with 4 chromic cat gut ligatures (4-0, Ethicon, Norderstedt, Germany), just proximal to the trifurcation, with a spacing of about $1 \mathrm{~mm}$. The length of the nerve thus affected is $4-5 \mathrm{~mm}$. The ligatures were tied in such a way that the diameter of the nerve is barely constricted (when viewed with $40 \mathrm{x}$ magnification). This degree of constriction retards but does not arrest flow in the superficial epineurial vasculature. The wounds were closed in layers with mersilene muscle sutures (5-0, Ethicon) and mersilene skin sutures (2-0, Ethicon). 


\section{2.b Methods}

\section{Microcirculatory investigative techniques}

\section{Laser Doppler flowmetry}

Skin blood flow (SBF) was measured non-invasively in the rat hind paw by means of the laser Doppler flowmetry (LDF) technique which has been described in detail in the first part of this chapter. In human skin, the penetration depth of the laser light contributing to the signal is about $0.6 \mathrm{~mm}^{530}$. In a pilot study in 4 rats, we measured the thickness of the skin in a biopsy which was obtained at the standard site of blood flow measurement (see below). Two biopsies were obtained 3 weeks after ipsilateral loose sciatic nerve ligation, whereas the two remaining biopsies were obtained from age-matched control animals. Skin thickness always exceeded 0.9 $\mathrm{mm}$. Hence, in our experimental set-up in rats blood cell movements were most likely recorded solely from the microvasculature of the skin, assuming similar properties as in man.

Measurements were executed with the Perimed PF3 (Perimed, Linköping, Sweden). The calibration procedures and settings were identical to those used in the human studies. Again, the output was digitised and saved on hard disk following which off-line analysis was performed, employing a software programme which was developed in our institute.

For assessment of skin blood flow, all animals were anaesthetised in the same way as for the operative procedure described above. Fig. 4.4 illustrates the experimental set-up.

Throughout the measurements body temperature was kept at $37^{\circ} \mathrm{C}$, using an infra-red heating lamp controlled by a thermo-analyser system (Hugo-Sachs Elektronik, MarchHugstedden, Germany). Ambient temperature was maintained at $21-23^{\circ} \mathrm{C}$. To measure skin blood flow, each animal was placed in the prone position on a plastic floor, which was adjustable in height. The plantar surface of the hind paws was inmobilised with adhesive tape. The abdomen of the rat was placed over an opening in the plastic floor with a diameter of 5 centimetres, through which cooling could be applied over a standardised area of abdominal skin. Laser Doppler Flowmetry (LDF) measurements were performed at a prominence located on the lateral side of the plantar surface of the rat hind paw. Within this specific area, the probe was moved about in order to determine the maximal basal skin blood flow. The skin area under investigation is autonomically innervated by the tibial nerve, which originates from the sciatic nerve ${ }^{500}$. Moreover, it has been demonstrated that activation of the sympathetic nervous system influences skin blood flow at the plantar surface of the rat hind paw ${ }^{556}$. Hence, sympathetic dysfunction due to the constriction injury of the sciatic nerve, if any, should result in blood flow abnormalities within the area under investigation. A micromanipulator (Leitz 115001, Leica BV, Rijswijk, The Netherlands) was used to position the standard angled probe close to the surface of the site of measurement. After stabilisation of the LDF signal for at least three minutes, basal skin blood flow was measured during three minutes.

In some groups of rats, we additionally assessed changes in the LDF signal following different experimental procedures.

We applied cooling of the rat abdomen by spraying ethyl-chloride (Medica BV, 's Hertogenbosch, The Netherlands) during 5 seconds (cold pressor test, CPT). Linderoth and colleagues $^{328}$ demonstrated that this coolling procedure provokes cutaneous vasoconstriction in the hind limbs of anaesthetised rats, as indicated by a decrease in skin blood flow. This 
vasoconstrictor response to the cold pressor test was impaired in rats subjected to lumbar sympathectomy ${ }^{328}$. Ergo, impairment of the vasoconstrictor response to the CPT is indicative of decreased sympathetic vasoconstrictor outflow ${ }^{328}$. After the CPT, skin blood flow was monitored during an additional three minutes. In all experiments, skin blood flow had reached its lowest value within these three minutes.

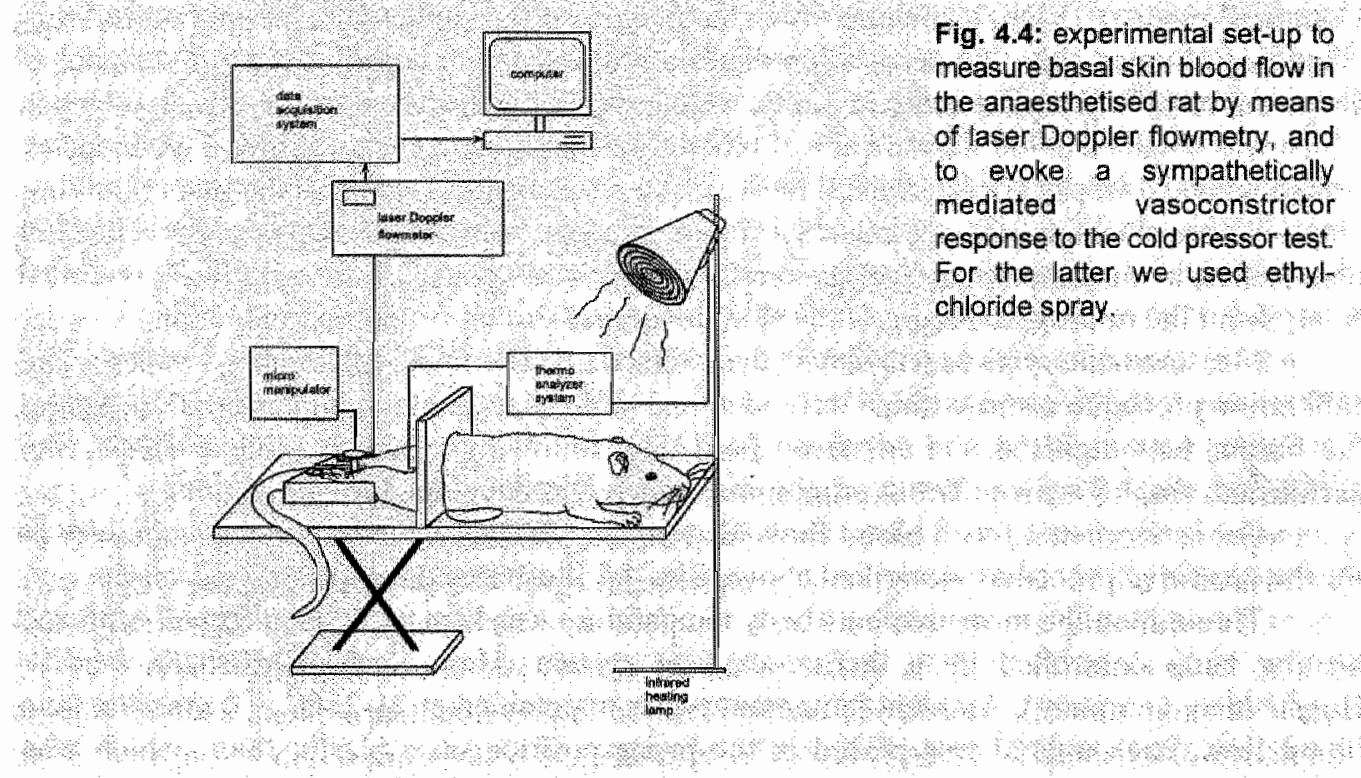

In other groups of rats, we assessed the influence of blockade of sensory and/or non-sensory nerve fibres on skin blood flow in the rat hind paw:

Capsaicin (Sigma Chemical Company, St. Louis, Missouri, USA) was applied perineurally at midthigh level ${ }^{174}$ in order to abolish profoundly and apparently irreversibly the capability of sensory fibres to mediate a neurogenic inflammatory response ${ }^{162,250}$. To prevent systemic effects, a thin layer of plastic foil was placed underneath a segment of the exposed sciatic nerve, after which a piece of cotton containing the capsaicin $1 \%$ solution was applied to this nerve. In several electrophysiological studies it has been shown that the neurotoxic effects of capsaicin on impulse propagation are specific to primary afferents nerve fibres ${ }^{26,213}$. Besides, it has been demonstrated that capsaicin does not exert effects on nonsensory (i.e. autonomic and motor) nerve fibres ${ }^{173,250}$. The cotton containing the capsaicin solution was removed after 15 minutes, after which excess capsaicin solution was absorbed with a dry piece of cotton ${ }^{321}$.

Lidocaine $2 \%$ (AUV, Cuijk, The Netherlands) was applied to the rat sciatic nerve in order to achieve non-selective and total blockade of impulse propagation ${ }^{126,205}$. To this end, part of the sciatic nerve was continuously (over a 1 hour period) covered with a layer of this solution. We opted for a $2 \%$ lidocaine concentration to ensure total blockade of impulse propagation. The total amount of lidocaine used never exceeded $1 \mathrm{ml}$. 
A 2\% lidocaine solution was applied to capsaicin-pretreated sciatic nerves to block selectively impulse propagation of nonsensory nerve fibres. Lidocaine has been demonstrated previously to block not only sensory but also nonsensory (motor and sympathetic) nerve fibres 185,278 . Besides, it has been demonstrated in isolated rat sciatic nerve that perineural capsaicin application potentiates the effect of subsequent lidocaine application ${ }^{470}$. Since so far no evidence has been presented for the involvement of other than sensory and sympathetic nerve fibres in neurogenic skin vasomotor control, the effect of lidocaine application to capsaicin pretreated nerves on skin blood flow abnormalities most likely can be attributed to blockade of impulse propagation of sympathetic nerve fibres. Again, lidocaine was applied to the nerve in such a way that part of the nerve was continuously (over a 60 minutes period) covered with a layer of this solution. The total amount of lidocaine used never exceeded $1 \mathrm{ml}$.

\section{Skin temperature}

Skin temperature was measured non-invasively in the rat hind paw by means of an electronic thermometer ( $78214 \mathrm{C}$, Hewlett Packard) attached to a circular metal thermocouple $\left(0.8 \mathrm{~cm}^{2}\right)$. The technique has been described in detail in the first part of this chapter. Again, the output was digitised and saved on hard disk following which off-line analysis was performed, employing a software programme which was developed in our institute.

Assessment of skin temperature was performed immediately prior to assessment of skin blood flow by means of laser Doppler flowmetry. Hence, the experimental conditions were similar. A circular metal thermocouple with a diameter of $5 \mathrm{~mm}$ was taped to the plantar surface of the hind paw of the anaesthetised rats following which skin temperature was assessed over a 3 minutes period.

\section{Macrocirculatory investigative techniques}

\section{In vitro investigation of subcutaneous arteries}

Structural ${ }^{388}$ and functional ${ }^{402}$ aspects of sympathetic innervation as well as sensitivity to adrenergic agonists ${ }^{386}$ can be investigated adequately in subcutaneous arteries. This prompted us to study these aspects in arteries that had been isolated from both hind paws of rats with 3 week old chronic constriction injury of the right sciatic nerve as well as from the left hind paw of rats 3 weeks after a sham procedure, during which the right sciatic nerve was exposed but not ligated, had been performed. Immediately prior to isolation of the (most laterally located) subcutaneous artery from the plantar surface of the hind paw, the rats were killed by a sharp blow on the neck and exsanguinated. The isolated artery is innervated by the tibial nerve, which originates from the sciatic nerve ${ }^{500}$. Immediately after isolation, four sets of experiments were performed.

In the first series, intramural noradrenaline containing nerves were visualised with the use of glyoxylic acid ${ }^{330}$. A small segment of the isolated artery was rinsed in cold-Krebs solution directly after dissection out of the hind paw. This vessel segment was incubated for 30 minutes at $37^{\circ} \mathrm{C}$ in a phosphate buffered solution ( $\mathrm{pH}=7.2$ ) containing $2 \%$ glyoxylic acid and $10 \%$ sucrose. Following incubation the vessel segment was rinsed with phosphate buffer solution, stretched on an object glass and dried under a stream of room air for thirty minutes. The vessel segment was heated for four minutes at $100{ }^{\circ} \mathrm{C}$ after which pictures were taken 
using a fluorescence microscope (Nikon Diaphot, BA 470-DM 455 filter, Nikon FE2 camera). In order to examine mechanical properties, the remaining segment of the isolated arteries was mounted on two stainlessesteel wires with a diameter of $40 \mu \mathrm{m}$ and placed horizontally in a myograph (fig 4.5) between an isometric force transducer (Kistler Morse DSC 6, Seattle, Washington, U.S.A.) and a displacement device (Mitatoyo, Tokyo, Japan) for recording of isometric force development ${ }^{10 ?}$. The organ chamber was filled with Krebs-Ringer bicarbonate solution (KRB, composition in mM: $\mathrm{NaCl} 118.3, \mathrm{KCl} 4.7, \mathrm{CaCl}_{2} 2.5, \mathrm{MgSO}_{4}, 7 \mathrm{H}_{2} \mathrm{O} 1.2$, $\mathrm{NaHCO}_{3} 25.0$, and glucose 5.5 ), which was maintained at $37^{\circ} \mathrm{C}$ and gassed with $95 \% \mathrm{O}_{2} / 5 \%$ $\mathrm{CO}_{2}$. Beforte experimentation, all arteries were distended to their individual optimal lumen diameter, i.e. that yielding the maximal force development, by stepwise increases in diameter with intermittent exposure to $5-\mathrm{HT}(10 \mu \mathrm{M})$. Subsequent experimentation was performed at this optimal diameter.

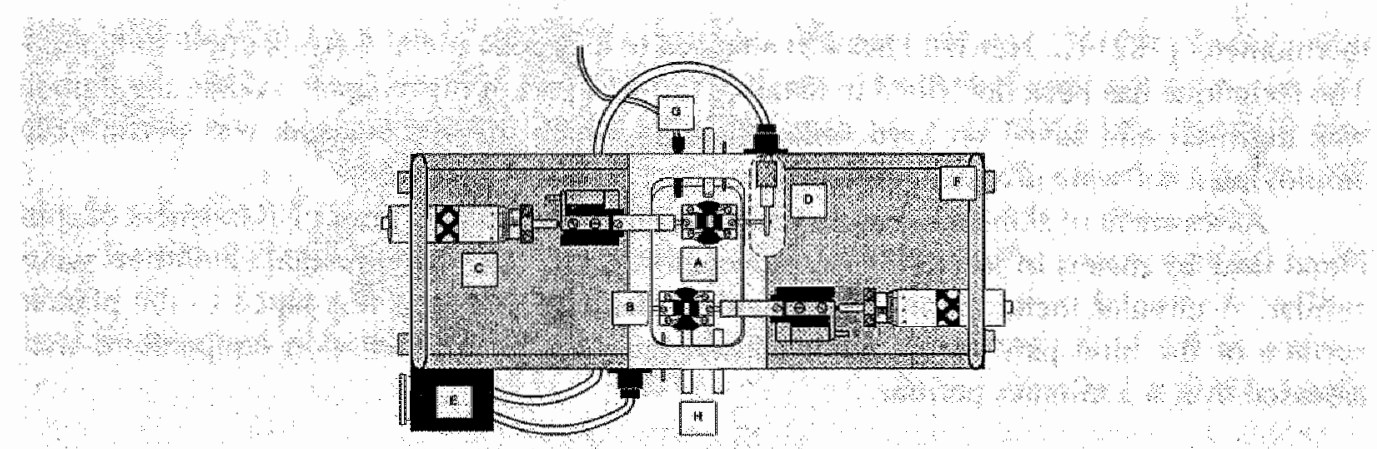

Rig 4.5 drawing of the myograph. The following cruclal parts can be distinguished a stainless steel organ bath with a volume of $10 \mathrm{ml}$ (A) in which wo preparations can be studied in parallel Two pair of stanless stealsupports (B), of which one is connected to a displacement device (C) and the other to a torce transducer (D) which is embedded in the Wall of the organ bath. The transdugers are connected ( $E$ ) to an amplifier and chalt recoirder The myographis either: Water or glectrically heated throeg h an internal cincult the actual termperature of the solution th the bath is reglistered by neans of a temperalure probe (G) Solutions in the bath can be aerated, removed, or added through several small lubes (H) which enter the bath from both sides, Adapted with the knd permission from Boonen:

In the second series, contractile responses to electrical field stimulation (EFS) were evaluated. Therefore, two platinum electrodes were placed along the longitudinal axis of the isolated vessel preparations. These electrodes were connected to a constant current source (amplitude $85 \mathrm{~mA}$ ) delivering electrical impulses of constant duration $(2 \mathrm{msec}$ ) at a variable frequency $(0.25$ to $32 \mathrm{~Hz})$. These conditions of electrical field stimulation (EFS) have been reported to selectively stimulate intramural nerves in isolated blood vessels ${ }^{146,388}$. In line with this, it was observed in preliminary experiments that EFS induced frequency-dependent contractions that could be abolished by $1 \mu \mathrm{M}$ prazosin, $1 \mu \mathrm{M}$ guanethidine or $1 \mu \mathrm{M}$ tetrodotoxin as well as by pretreatment with $0.3 \mathrm{mg} / \mathrm{ml}$ 6-hydroxy dopamine. Moreover, EFS applied in the presence of guanethidine (a procedure that is routinely used to evaluate perivascular sensory motor nerve function ${ }^{265}$ ) failed to reduce contractile responses induced by phenylephrine, endothelin, 
prostaglandin $\mathrm{F}_{2} \alpha$ or vasopressin. Collectively these observations indicate that vascular responses to EFS are exclusively due to stimulation of intramural adrenergio nerves.

In the third series, contractile responses to cumulative doses of various adrenergic agonists were assessed. Concentration-response curves were obtained by increasing the concentrations in half- $\log$ increments. Sensitivities to the agonists $\left(\mathrm{pD}_{2}=-\log \mathrm{EC}_{50}\right.$, where the $\mathrm{EC}_{50}$ is the agonist concentration needed to produce $50 \%$ of the maximal response) were calculated by interpolation on a logistic curve fit of the individual concentration response curves (Graphpad Inplot 3.01, Institute for Scientific Information, San Diego, CA, USA). Concentration response curves $(10 \mu \mathrm{M}-30 \mu \mathrm{M})$ were constructed for the non-selective adrenoceptor agonist noradrenaline, the $\alpha_{1}$-selective agonist phenylephrine and the $\alpha_{2}-a_{g o n i s t}$ B-HT 933 (azepexole). The ratio of the maximal response to EFS and the maximal response to noradrenaline was calculated as a functional measure of adrenergic innervation. B-adrenergic responses were investigated by constructing concentration-response curves for isoprenaline after precontracting the arteries with $35 \mathrm{mM} \mathrm{K}^{+}$. Concentration-response curves for isoprenaline were constructed and maximal relaxing responses were expressed as percentage of the precontraction. Initially, vasoconstrictor (noradrenaline, phenylephrine, B-HT 933) and vasodilator (isoprenaline) responses to these adrenoceptor agonists were assessed without blockade of presynaptic reuptake of catecholamines. In order to investigate the efficacy of the latter mechanism, we subsequently studied the influence of $3 \mu \mathrm{M}$ cocaine (which blocks presynaptic reuptake of catecholamines) on the sensitivity of arteries for noradrenaline $(10 \mu \mathrm{M}$ $-30 \mu \mathrm{M}$ ). All drugs used were obtained from Sigma Chemicals (St. Louis, MO, USA)

In the fourth series, we studied the structural dimensions of vessel segments in which contractile responses to EFS and various pharmacological agents had been assessed. To this end, these vessel segments were fixed at their optimal diameter in phosphate buffered (pH 7.4) formaldehyde ( $4 \%$ ) immediately after evaluation of contractile responses. Preparations were paraffin embedded and cross sectioned using standard histological techniques ${ }^{106}$. Cross sections (4 $\mu \mathrm{m}$ ) were stained for elastin using Lawson's solution (Boom B.V., Meppel, The Netherlands). Video images were generated from the cross sections using a Zeiss Axioscope (Zeiss, Germany) and a standard CCD camera (Stemmer, Germany). With the use of a software package for personal computers (Jandel Scientific Video Analysis Software, (JAVA V 1.21), Jandel Scientific, Corte Madera, CA, USA), the internal and external medial circumference were measured, which were respectively demarcated by the internal and external elastic lamina. From these, assuming circular vessel conformation, the lumen area and the total area could be derived $^{106}$. Medial cross sectional area (CSA) was obtained by subtracting lumen area from the total area. Internal lumen radius ( $\mathrm{r}$ ) was calculated from the internal circumference, again assuming a circular vessel conformation. From these parameters, average media thickness $\left(\mathrm{M}_{\nu}\right)$ was calculated using the following equation.:

$$
M_{\gamma}=r+\sqrt{r^{2}+\frac{C S A}{\pi}}
$$

Finally, the wall to lumen ratio (W/L) was calculated by dividing twice the average media thickness by the optimal lumen diameter. 


\section{2.c Methodological considerations}

Various experimental factors necessitated supplementary methodological measures and/or additional experiments.

Skin blood flow was measured in unoperated rats at identical instants as in nerve-injured rats ( 1 day before ligation as well as $1,3,5,7,14,21$, and 28 days after the operative procedure) in order to control for potentiall effects of repeated measurements and increasing age/weight.

In rats without nerve injury, LDF was employed to obtain information on the effects on SBF of capsaicin and lidocaine application to nonligated sciatic nerves. To this end, capsaicin and lidocaine were applied consecutively, as described before.

Potential effects of lidocaine application on SBF abnormalities induced by loose sciatic nerve ligation may result not only from direct effects of this substance on the treated nerve, but also from systemic effects. To exclude the latter, we performed the following experimental protocol. SBF was assessed before and at some instants after loose sciatic nerve ligation. After ligation, SBF was assessed before and after creation of a surgical wound in the left fore paw of the rats. To this end, the triceps brachii muscle was dissected bluntly. The size of this surgical wound in the fore paw was comparable to the size of the surgical wound in the hind paw used for the application of capsaicin and lidocaine to the sciatic nerve. Lidocaine was then applied in such a way that a layer of the solution was continuously present in this wound over a 60 minutes period. The total amount of lidocaine used was $1 \mathrm{ml}$ for each experiment.

We applied chemical conduction blockades instead of surgical transection of nerves (sympathectomy and/or rhizotomy) for several reasons. Firstly, the use of chemical conduction blockades enabled us to assess continuously, before and after application of the nerve conduction blockade, skin blood flow while the probe of the laser Doppler flowmeter remained in the same position. Thus, spatial variation is prevented and temporal variation can be kept to a minimum. Secondly, cutting of damaged nerves has been reported to provoke prolonged afterdischarges, both proximally and distally ${ }^{424}$, which may influence skin blood flow readings.

\section{2.d Statistics}

Unless stated otherwise, the following statistical methods were used. Data about the presence of an abnormal posture of the hind paw are presented as prevalences. All other data are expressed as medians with their interquartile ranges, since the data obtained were not normally distributed. In some chapters, data are expressed relative to their control value, i.e., value of interest divided by the corresponding control (initial) value. Non-parametric analysis of variance was performed with the Friedman test, first for all instants together, and if significant, subsequently also for subgroups. In case two instants were compared, we used the Wilcoxonsigned ranks test for paired data. Differences were regarded statistically significant when $\mathrm{p}<$ 0.05 .

In chapter 13, data are expressed as means \pm standard error of the mean. Statistical significance of differences was evaluated by Student's $t$ test for paired or unpaired observations. The Fisher"s exact (Chi-square) test was used to test for statistical significance of differences in sympathetic nerve density. The Pearson correlation coefficient was determined to assess the strength of linear association between maximum contractile responses to electrical field stimulation and sensitivity to noradrenaline. $p<0.05$ was considered statistically significant. 


\section{Chapter 5}

\section{REFLEX SYMPATHETIC DYSTROPHY:}

\section{EVOLUTION OF MICROCIRCULATORY DISTURBANCES IN TIME}

HAJM Kurvers, MJHM Jacobs, RJ Beuk, FAJM van den Wildenberg, PJEHM Kitslaar, DW Slaaf, and RS Reneman

Departments of Surgery of the University Hospital Maastricht and of the Academic Medical Centre Amsterdam, The Netherlands.

Departments of Biophysics, Physiology and Surgery, Cardiovascular Research Institute Maastricht (CARIM), The Netherlands.

Based on: Pain, 1995, vol. 60, p. 333-340. 


\section{SUMMARY}

Blood flow in the affected extremity of reflex sympathetic dystrophy (RSD) patients has been reported to be disturbed in bone and skin. Since blood flow within these tissues is influenced by sympathetic tone, these findings suggest sympathetic dysfunction. We investigated whether there are disturbances in the sympathetic function of skin microcirculation at the 3 clinical stages of RSD. Laser Doppler flowmetry (LDF) was used to obtain information about total (mainly thermoregulatory) skin blood flow (TSBF), since blood flow in arteriovenous anastomoses and subpapillary plexus, which are richly innervated by sympathetic nerve endings, contributes predominantly to the flow signal as obtained by LDF. Capillary microscopy was used to appraise whether the trophic changes, as observed in RSD, result from an impaired nutritive skin blood flow (NSBF). Transcutaneous oximetry was employed as a measure of the oxygenation of superficial skin layers $\left(\mathrm{TC}_{\mathrm{OO}}\right)$. Skin temperature (ST) was also determined. Patients were divided into three clinical stages: stage I in case of a chronic warmth sensation, stage II in case of an intermittent warmth and cold sensation, and stage III in case of a chronic cold sensation. As compared to controls: 1) TSBF was increased at stage I and decreased at stages II and. III, 2) NSBF was decreased at stages II and III 3) $\mathrm{TC} p_{02}$ was not impaired at any stage 4) ST was increased at stage I and decreased at stage III. The present study is the first to report an inerease of thermoregulatory skin blood flow at stage I of RSD, which may be caused by a decrease in efferent sympathetic nerve impulses. At stage II and III both thermoregulatory and nutritive skin blood flow were found to be decreased, which may reflect increased sensitivity of skin microvessels to (circulating) catecholamines.

\section{INTRODUCTION}

Reflex sympathetic dystrophy (RSD) is a pain syndrome which often results from trauma to soft tissues or a nerve plexus ${ }^{460,464}$. It is characterised by autonomic, motor, and sensory disturbances ${ }^{60,50}$. In addition, trophic changes may appear at later stages of the syndrome ${ }^{528}$.

Blood flow in the affected extremity of RSD patients has been reported to be disturbed in bone ${ }^{12,533}$ as well as $\operatorname{skin}^{33,441,442}$. Since blood flow within these tissues is influenced by sympathetic tone, these findings may indicate sympathetic dysfunction. Both an increase $e^{57,114,333}$ and a decrease $\mathrm{e}^{138.19}$ in efferent sympathetic nerve impulses have been suggested. Alternatively, antidromic stimulation ${ }^{460}$ as well as an excessive inflammatory response ${ }^{194,398}$ have been purported to play a role in RSD.

We investigated microcirculatory changes at all clinical stages of RSD, in order to gain insight into the underlying pathophy siological mechanisms. Laser Doppler Flowmetry (LDF) was used to obtain information about skin microcirculation since the blood flow in arteriovenous anastomoses and the subpapillary plexus, which are richly innervated by sympathetic nerve endings $\mathrm{s}^{219,547}$, contribute predominantly to the flow signal obtained by LDF ${ }^{133}$. Capillary microscopy was used to appraise whether the trophic changes, which frequently appear at later stages of the RSD syndrome, result from an impaired nutritive skin blood flow. In addition transcutaneous oximetry was employed as a measure of the oxygenation of superficial skin layers. Skin temperature was also determined. 
PATIENTS AND METHODS (for detailed information the reader is referred to chapter 4)

\section{Patients}

The study was performed on 120 patients with RSD of the upper extrenity which all met the criteria described in chapter 4 . Additional disorders, evaluated by means of patient history and physical examination, are listed in table 5.1. RSD was precipitated by fractures (finger 7\%; carpal/metacarpal bone $6 \%$; wrist $27 \%$; elbow $6 \%$; shoulder $2 \%$ ), soft tissue traumas (finger $6 \%$; hand $7 \%$; wrist $5 \%$; lower arm $2 \%$; elbow $3 \%$ ), operative procedures (hand $5 \%$; wrist $2 \%$; elbow $1 \%$ ), neurovascular compressions (elbow $5 \%$; thoracic outlet $2 \%$; cervical spine $1 \%$ ), and cerebrovascular accidents (2\%). In $9 \%$ of all cases no precipitating event could be traced. Pain complaints were restricted to the fingers in 15 percent of all cases. Besides fingers, hand and wrist were involved in 18 and 31 percent of the cases, respectively. Pain complaints involved also the lower arm, upperarm, and shoulder in 14,6, and 16 percent of the cases, respectively. A group of 26 volunteers was used as controls. Smoking habits, age, and duration of disease are shown for each stage in table 5.2.

\begin{tabular}{|c|c|c|c|}
\hline STAGE OF DISEASE & $\|$ & II & IIII \\
\hline $\mathbf{N}$ & 22 & 70 & 28 \\
\hline $\begin{array}{l}\text { AUTONOMIC } \\
\text { edema } \\
\text { increase ediema on dependency } \\
\text { hyperhidrosis } \\
\text { abnormal nail growth } \\
\text { abnormal hair growth }\end{array}$ & $\begin{array}{l}95 \\
41 \\
68 \\
50 \\
36\end{array}$ & $\begin{array}{l}50 \\
63 \\
69 \\
51 \\
26\end{array}$ & $\begin{array}{l}64 \\
57 \\
57 \\
46 \\
25\end{array}$ \\
\hline $\begin{array}{l}\text { TROPHIC } \\
\text { skin atrophy } \\
\text { subcutaneous atrophy } \\
\text { abnormal consistency nails }\end{array}$ & $\begin{array}{l}14 \\
5 \\
27\end{array}$ & $\begin{array}{l}33 \\
26 \\
46\end{array}$ & $\begin{array}{l}61 \\
61 \\
46\end{array}$ \\
\hline $\begin{array}{l}\text { MOTOR } \\
\text { reduced strength } \\
\text { muscle spasm } \\
\text { muscle wasting } \\
\text { tremor } \\
\text { incoordination } \\
\text { involuntary movements }\end{array}$ & $\begin{array}{l}86 \\
32 \\
18 \\
45 \\
95 \\
23\end{array}$ & $\begin{array}{l}100 \\
21 \\
46 \\
51 \\
86 \\
17\end{array}$ & $\begin{array}{l}96 \\
54 \\
61 \\
68 \\
82 \\
36\end{array}$ \\
\hline $\begin{array}{l}\text { SENSORY } \\
\text { increase pain after exercise } \\
\text { hyperalgesia } \\
\text { mechanical allodynia } \\
\text { thermal allodynia (warmth) } \\
\text { thermal allodynia (cold) } \\
\text { hypesthesia } \\
\text { paresthesia }\end{array}$ & $\begin{array}{l}95 \\
86 \\
59 \\
55 \\
41 \\
55 \\
27\end{array}$ & $\begin{array}{l}94 \\
89 \\
73 \\
49 \\
57 \\
66 \\
59\end{array}$ & $\begin{array}{l}89 \\
86 \\
68 \\
54 \\
79 \\
71 \\
71\end{array}$ \\
\hline
\end{tabular}

Table 5.1: incidence of autonomic, trophic, motor, and sensory features at the various stages, expressed as percentage of the whole group. 


\begin{tabular}{lllll}
\hline STAGE & N & $\begin{array}{c}\text { smokers } \\
(\%)\end{array}$ & $\begin{array}{c}\text { Age in years } \\
\text { mean (range) }\end{array}$ & $\begin{array}{l}\text { Duration of disease in months } \\
\text { mean (range) }\end{array}$ \\
\hline Controls & 26 & 31 & $48(21-69)$ & \\
I & 22 & 50 & $51(24-74)$ & $2(1-22)$ \\
II & 70 & 46 & $48(18-91)$ & $8(1-59)$ \\
III & 29 & 41 & $48(16-77)$ & $33(2-120)$ \\
\hline
\end{tabular}

Table 5.2: smoking habits, age, and duration of disease per group.

\section{Experimental protocol}

Capillary microscopy was performed, with the hand at heart level, in the most symptomatic finger of RSD patients. Images of capillaries were recorded. Subsequently, arterial occlusion was induced with a cuff around the upperarm. After acute deflation of the cuff, images were recorded during reactive hyperaemia. Subsequently, we synchronously performed laser Doppler flowmetry, transcutaneous oximetry, and skin temperature. The laser Doppler probe holder was attached to the most symptomatic finger. LDF measurements were performed at unheated skin. Skin temperature was measured at the dorsum of the same digit. Transcutaneous oximetry $\left(\mathrm{TC} p_{02}\right)$ was performed at the dorsum of the hand immediately adjacent to the most symptomatic finger. T $\mathrm{c}_{\mathrm{O} 2}$ measurements were performed at a local skin temperature of $44^{\circ} \mathrm{C}$ in order to obtain maximal suppression of local vasoconstrictor mechanisms. The values thus acquired, represent the arteriolar $P_{\mathrm{O} 2}{ }^{87} . \mathrm{LDF}$ and $\mathrm{TC} p_{\mathrm{O} 2}$ were assessed under resting conditions and during reactive hyperaemia following arterial occlusion. From the laser Doppler flow readings we obtained the mean flow under resting conditions (LDFr; expressed in perfusion units: PU) and the peak flow during reactive hyperaemia (LDFp, PU). The morphologic parameters obtained (off-line) from the capillary video microscopy recordings were the capillary density (CDe, $\mathrm{mm}^{-2}$, defined as the number of erythrocyte-perfused capillaries per square millimetre of nailfold skin) and the capillary diameter (CDi, in $\mu \mathrm{m}$, represented by the width of the red blood cell column, measured halfway the arteriolar limb), The haemodynamic parameters determined were the red blood cell Velocity under resting conditions ( $\mathrm{RBCVr}$, $\mu \mathrm{m} / \mathrm{s}$ ) and at peak of reactive hyperaemia $(\mathrm{RBCVp}, \mu \mathrm{m} / \mathrm{s})$. From these measured parameters, we additionally derived some other parameters. Firstly the transport capacity index (TCI) which is considered to be a measure of the total capillary blood transport capacity per unit surface area (calculated as the product of average capillary cross-sectional area and capillary density: $\pi$ * average $\left.(\mathrm{Cdi} / 2)^{2} * \mathrm{Cde}\right)$. Secondly the volume flow per unit surface area (VOLF, $\left.\mu \mathrm{m} / \mathrm{s}\right)$ which is considered to be a measure of the nutritive volume flow through superficial capillaries per square millimetre of nailfold skin (calculated as $\pi *$ average $(\mathrm{Cdi} / 2)^{2} * \mathrm{Cde} * \mathrm{RBCVr}$ ). From the $T C p_{02}$ readings we obtained skin oxygen tension at rest $\left(\mathrm{TC} p_{\mathrm{O}_{2}} \mathrm{r}, \mathrm{mm} \mathrm{Hg}\right.$ ) and the peak value during reactive hyperaemia $\left(\mathrm{TC} \mathrm{p}_{\mathrm{O} 2} \mathrm{p}, \mathrm{mm} \mathrm{Hg}\right)$. 


\section{RESULTS}

\section{Laser Doppler flowmetry}

The results obtained in the controls and in the three patient groups are summarised in table 5.3 . As compared to controls, blood flow at rest (LDFr) was higher at stage I $(p<0.05)$ and lower at stages II ( $p<0.05)$ and III $(p<0.001)$. LDFr was also higher at stage I as compared to stages II $(p<0.01)$ and III $(p<0.0001)$. As compared to controls, maximum skin blood flow during reactive hyperaemia (LDFp) was higher at stage $I(p<0.05)$ and lower $(p<0.05)$ at stage III. LDFp was higher at stage I as compared to stages II $(p<0.01)$ and III $(p<0.001)$. Linear regression analysis revealed a decrease in both $\operatorname{LDFr}(\mathrm{Y}=77-(0.76 *$ duration of disease); $95 \% \mathrm{CI}=-1.3$ to $-0.3 ; \mathrm{R}=0.24)$ (fig. 5.1) and LDFp ( $\mathrm{Y}=123-(0.78 *$ duration of disease); $95 \% \mathrm{CI}=-1.4$ to $-0.2 ; \mathrm{R}=0.24)$ as a function of duration of the disease.

\section{Capillary microscopy}

The results obtained in the control group and in the three patient groups are summarised in table 5.4. Capillary diameters did not differ between controls and patients. Capillary diameter was larger $(p<0.05)$ at stage III as compared to stage II, and tended to be larger $(p=0.08)$ at stage III as compared to stage I. Capillary density did not differ, neither between patient groups and controls, nor between patient groups. Both capillary diameter and capillary density did not change as a function of duration of the disease. As compared to controls, red blood cell velocity at rest $(\mathrm{RBCVr})$ was lower at stages II $(\mathrm{p}<0.05)$ and III $(\mathrm{p}<0.001)$. No differences were observed between controls and stage I patients. $\mathrm{RBCV}_{\mathrm{r}}$ was also lower at stage III as compared to stages II ( $\mathrm{p}<0.01)$ and I $(\mathrm{p}<0.0001)$ (fig. 5.2). As compared to controls, peak red blood cell velocity during reactive hyperaemia $(\mathrm{RBCVp})$ was lower at stage III $(\mathrm{p}<0.05)$. RBCVp was also lower at stage III as compared to stages I $(p<0.0001)$ and II $(p<0.001)$. Besides, RBCVp was lower $(p<0.05)$ at stage II as compared to stage I. Regression analysis revealed a linear decrease in both $\mathrm{RBCVr}(\mathrm{RBCVr}=308-(2.77 *$ duration of disease $) ; 95 \% \mathrm{CI}=-5.1$ to -0.5 ; $\mathrm{R}=0.18$ ) (fig. 5.2) and $\mathrm{RBCVp}\left(\mathrm{RBCVp}=810-\left(4.56^{*}\right.\right.$ duration of disease); $95 \% \mathrm{Cl}=-8.3$ to $-0.8 ; R=0.20$ ) as a function of duration of the disease, independent of the disease stage. The anatomical blood transport capacity (TCI) did not differ, neither between patient groups and controls, nor between the three patient groups. As compared to controls, nutritive skin blood flow per square millimetre of nailfold skin (VOLF) was lower at stages II $(p<0.01)$ and III ( $p$ $<0.01)$. Compared to stage I, VOLF was also lower at stages II $(p<0.05)$ and III $(p<0.0001)$.

\section{Transcutaneous oximetry}

The data acquired in controls and the three patient groups are summarised in table 5.3. As compared to controls, skin oxygen tension at rest $\left(\mathrm{TC} p_{\mathrm{O} 2} \mathrm{r}\right)$ was higher $(\mathrm{p}<0.05)$ at stage III. No differences were observed between stages I and II on the one hand and controls on the other. As compared to controls, peak skin oxygenation $\left(\mathrm{TC} p_{\mathrm{O} 2} \mathrm{p}\right)$ tended to be higher $(\mathrm{p}=0.08)$ at stage III. Linear regression analysis did not reveal changes in $\mathrm{TC} p_{\mathrm{O}_{2}} \mathrm{r}$ and $\mathrm{TC} p_{\mathrm{O} 2} p$ as a function of duration of the disease. 


\begin{tabular}{|c|c|c|c|c|}
\hline & controls & stage I & stage II & stage III \\
\hline LDFr & $\begin{array}{l}70 \\
(50-97)\end{array}$ & $\begin{array}{l}107 \\
(73-178)\end{array}$ & $\begin{array}{l}55 \\
(36-72)\end{array}$ & $\begin{array}{l}29 \\
(15-53)\end{array}$ \\
\hline LDFp & $\begin{array}{l}111 \\
(84-144)\end{array}$ & $\begin{array}{l}150 \\
(95-218)\end{array}$ & $\begin{array}{l}95 \\
(70-140)\end{array}$ & $\begin{array}{l}77 \\
(53-116)\end{array}$ \\
\hline $\mathrm{TCPO} 2 \mathrm{r}$ & $\begin{array}{l}60 \\
(49-72)\end{array}$ & $\begin{array}{l}62 \\
(53-72)\end{array}$ & $\begin{array}{l}66 \\
(56-72)\end{array}$ & $\begin{array}{l}66 \\
(62-81)\end{array}$ \\
\hline TCPO2p & $\begin{array}{l}67 \\
(54-75)\end{array}$ & $\begin{array}{l}69 \\
(61-76)\end{array}$ & $\begin{array}{l}69 \\
(60-79)\end{array}$ & $\begin{array}{l}74 \\
(64-78)\end{array}$ \\
\hline $\begin{array}{l}\text { Skin } \\
\text { temp. }\end{array}$ & $\begin{array}{l}31.6 \\
(31.0-33.1)\end{array}$ & $\begin{array}{l}33.4 \\
(32.2-33.8)\end{array}$ & $\begin{array}{l}32.4 \\
(31.0-33.2)\end{array}$ & $\begin{array}{l}30.8 \\
(29.2-32.2)\end{array}$ \\
\hline
\end{tabular}

Table 5.3: data obtained by laser Doppler flowmetry, transcutaneous oximetry, and thermography expressed as medians and interquartile ranges, for both individual patient groups and controls. $L D F r=m e a n$ total skin blood flow under resting conditions; LDFp = total skin blood flow at peak of reactive hyperaemia; $T C P O 2 r=$ mean skin oxygenation under resting conditions; $T C P O 2 p=$ skin oxygenation at peak of reactive hyperaemia.

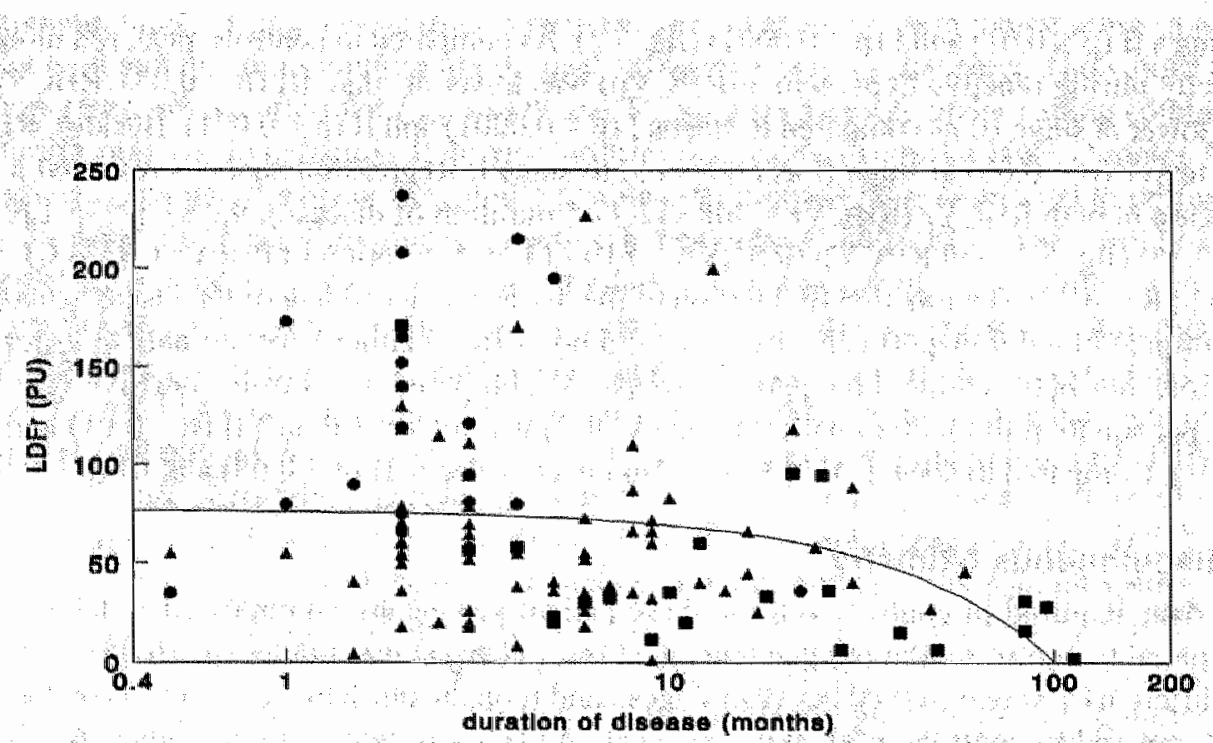

Fig. 5.1. relationship between laser Doppler flow under resting conditions and duration of disease yielded the following linear regression equation: $Y=77-(0.76 * X) 95 \% \mathrm{Cl}$ slope: -1.3 to $-0.3 ; R=0.24$. On the $X$-axis is plotted the duration of disease in months (logaritmic scale), and on the y-axis thermoregulatory skin blood fiow at rest (in PU). Indicates stage $\|$ patients; $\mathbf{A}$ indicates stage li patients; $\mathbf{m}$ indicates stage III patients. 


\begin{tabular}{lllll}
\hline & controls & stage & stage II & stage III \\
\hline Cde & $50(43-62)$ & $51(39-61)$ & $52(39-61)$ & $54(37-61)$ \\
Cdi & $11(9-12)$ & $10(8-11)$ & $8(8-11)$ & $11(9-13)$ \\
RBCVr & $318(226-453)$ & $367(240-525)$ & $192(105-427)$ & $90(45-237)$ \\
RBCVp & $762(581-1085)$ & $884(763-1147)$ & $702(542-1025)$ & $442(338-695)$ \\
TCl & $4.4(3.1-5.5)$ & $3.6(2.3-4.8)$ & $3.9(2.3-5.2)$ & $4.6(3.3-6.1)$ \\
VOLF & $1511(911-2027)$ & $1127(752-2079)$ & $682(311-1321)$ & $432(247-1094)$ \\
\hline
\end{tabular}

Table 5.4: data obtained by capillary microscopy expressed as medians and interquartile ranges, for both individual patient groups and controls. $\mathrm{Cde}=$ capillary density; $\mathrm{Cdi}=$ capillany diametter: $\mathrm{RBCV} / \mathrm{r}=\mathrm{red}$ blood cell velocity under resting conditions; $R B C V p=$ red blood cell velocity at peak of reactive hyperaemia; $T C l$ $=$ transport capacity index per unit surface area $(* 1000) ;$ VOLF $=$ volume flow per unit surface area $(* 1000)$.

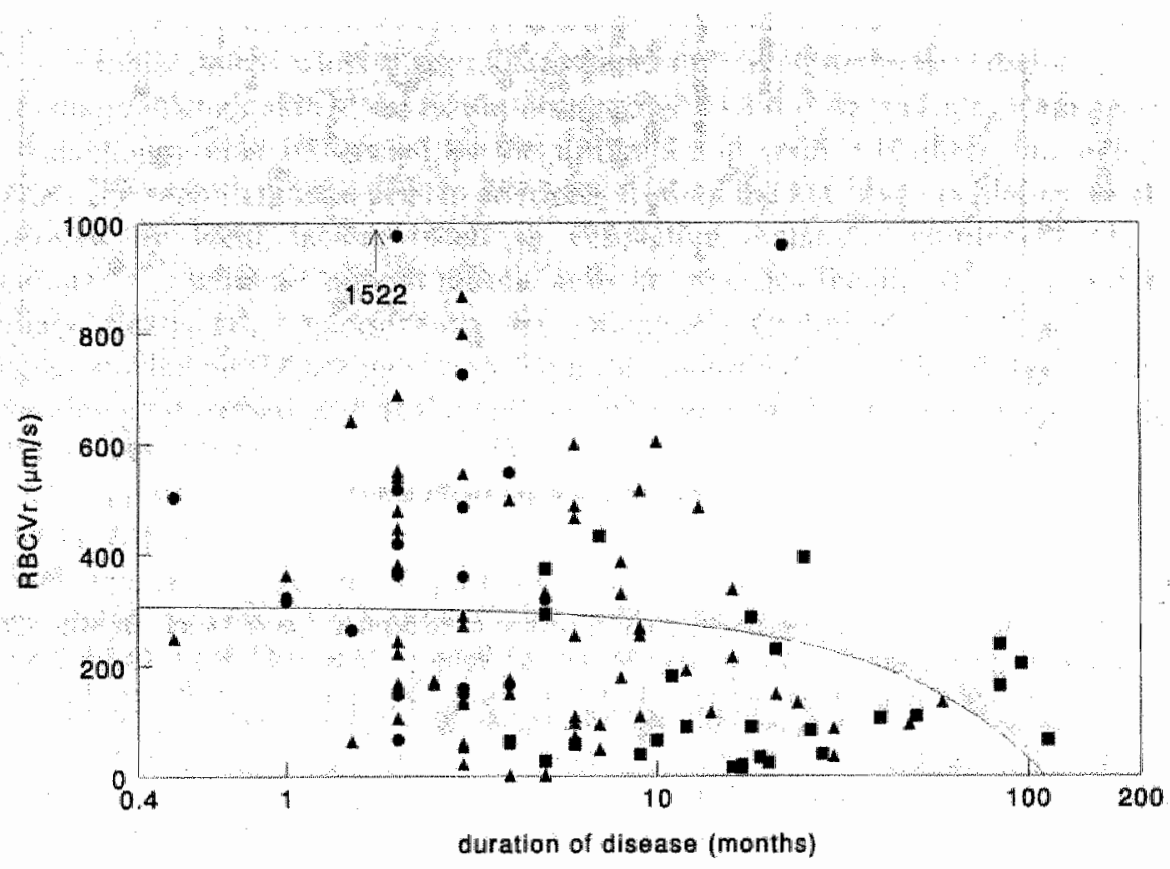

Fig. 5.2: relationship between red blood cell velocity under resting conditions and duration of disease yielded the following linear regression equation: $Y=308-(2.77 \times X) ; 95 \% \mathrm{Cl}$ slope: -5.1 to $-0.5 ; \mathrm{R}=0.18$. On the $x$ axis is plotted duration of disease in months (logarithmic scale), and on the $y$-axis red blood cell velocity at rest (in $\mathrm{Hm} / \mathrm{s}$ ). indicates stage I patients; $\boldsymbol{\Lambda}$ indicates stage II patients; $\mathbf{m}$ indicates stage III patients 


\section{Skin temperature}

The results obtained in controls and in the three patient groups are summarised in table 5.3. As compared to controls, skin temperature was higher $(\mathrm{p}<0.01)$ at stage I and lower $(\mathrm{p}<0.05)$ at stage III. Skin temperature was also lower at stage III as compared to stages $I(p<0.0001)$ and II $(\mathrm{P}<0.01)$. Linear regression analysis revealed a decrease in skin temperature as a function of duration of the disease $(\mathrm{Y}=32.2-(0.03 *$ duration of disease $) 95 \% \mathrm{CI}=-0.05$ to $-0.01 ; \mathrm{R}$ $=0.18$ ) (fig. 5.3).

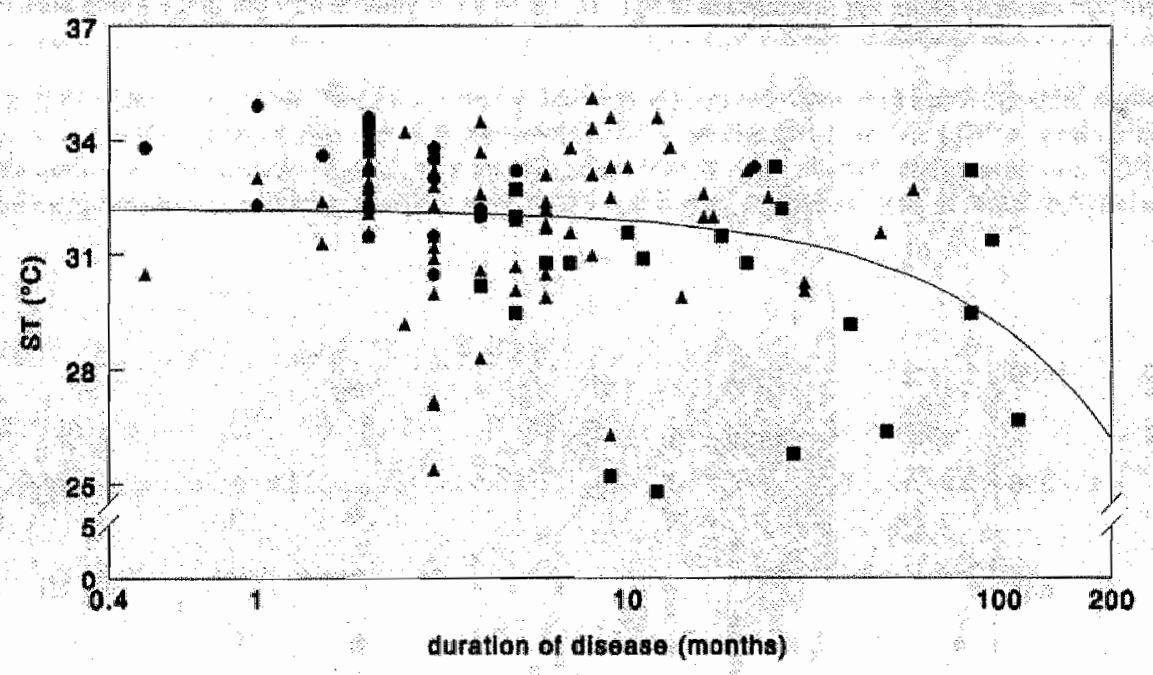

Fig. 5.3. relationsthip between skin temperature under resting conditions and duration of disease yielded the following regression equation, $Y=322-(0.03 * X) ; 95 \% \mathrm{Cl}$ slope -0.05 to $-0.01, \mathrm{R}=0.18$, On the $X$-axis is plotted the duration of disease in months (logarithmic scale), and on the y-axis skin temperature at rest (in

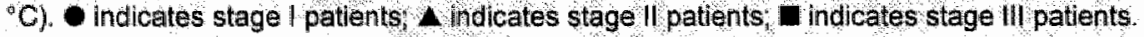




\section{DISCUSSION}

The present study shows that in RSD patients, as compared to controls, thermoregulatory skin blood flow is increased at stage I and decreased at stages II and III. Since sympathectomy in humans has been reported to cause an increase in skin blood flow ${ }^{314,510}$, the observed increase in thermoregulatory skin blood flow may be caused by a decrease in efferent sympathetic nerve impulses. Since such a decrease has been reported to induce increased sensitivity of vascular structures to catecholamines ${ }^{36,42}$, the observed decrease in total skin blood flow at stages II and III of the disease may result from hypersensitivity to (circulating) catecholamines of supplying skin microvessels. Alternatively, an increase in efferent sympathetic nerve impulses through a somato-sympathetic reflex may account for the observed decrease in total skin blood flow at stages II and III. The findings of Drummond and colleagues ${ }^{138}$, however, who observed a decrease in venous plasma levels of noradrenaline collected from the affected limb in RSD patients, argue against the latter hypothesis. Moreover, Arnold and colleagues ${ }^{19}$ presented direct in-vivo evidence for supersensitivity of venous $\alpha$-adrenoceptors to noradrenaline in limbs affected by RSD. On the basis of the findings in our study, however, we can not exclude the possibility that antidromic mechanisms contribute to the observed increase in thermoregulatory skin blood flow at stage 1 , since it has been demonstrated previously, in both humans ${ }^{392}$ and rats $^{174}$, that mechanical stimulation of peripheral nerves provokes dilatation of skin microvessels through antidromic mechanisms at sites distant from the site of nerve stimulation.

Unlike thermoregulatory skin blood flow, nutritive skin blood flow was not increased at stage I. This discrepancy may be caused by the difference in control of these microcirculatory subsystems. The smooth muscle cells in arterioles feeding the nutritive capillaries are likely to be controlled by local factors (such as vasoactive metabolic substances and tissue oxygenation $)^{519,547}$, whereas smooth muscle cells in arterioles feeding AV anastomoses and subpapillary plexus are predominantly sympathetically controlled ${ }^{519,547}$. Besides, the AV anastomoses and the plexus are also sympathetically controlled ${ }^{519,547}$. The decrease in nutritive skin blood flow as observed at stage II and III has been reported before ${ }^{441,442}$, and coincides with a decrease in total skin blood flow. Therefore, this decrease may be explained by constriction of arterioles supplying both thermoregulatory and nutritive subsystems. If this tendency towards an impaired nutritive microcirculation persists, ultimately trophic changes may develop in the skin and subcutaneous tissues in a majority of stage III RSD patients, as is the case in our study.

In general, the clinical course of RSD is divided into three stages: an acute, a dystrophic, and an atrophic stage ${ }^{52,447}$. This clinical course, however, is rather variable from patient to patient. In addition, there is a considerable overlap of signs and symptoms between these three stages, which makes it virtually impossible to classify the patients unequivocally. In contrast, categorisation on the basis of the patients' perception of skin temperature, as was done in our study, was found to be simple and reproducible, and corresponds roughly with the duration of the disease.

Skin blood flow abnormalities corresponded better with stage of the disease than with duration of the disease. The observed large interindividual differences with respect to the abnormalities in skin blood flow may reflect dissimilar rates with which pathophysiological processes take place. Moreover, categorisation on the basis of the patients' perception of skin temperature may take into account the actual regulatory state at the time of investigation, which 
is of importance because in RSD patients skin temperature (and thus skin blood flow) has been reported to demonstrate short-term fluctuations ${ }^{58}$. These short-term fluctuations in skin temperature may result from a dynamic interplay between opposing vasomotor effects (vasoconstrictor sympathetic versus vasodilator antidromic mechanisms).

Oxygenation of the superficial skin layers at $44^{\circ} \mathrm{C}$ was not impaired at any stage of the disease, which is in contrast with the observed impairment of nutritive skin blood flow at stages II and III: This contrast may result from the fact that transcutaneous oximetry was performed at a probe temperature of $44^{\circ} \mathrm{C}$ thus overriding local vasoconstrictive mechanisms, whereas assessment of nutritive skin blood flow took place at unheated skin. Under normal environmental conditions, in which the affected tissues are colder, the impaired nutritive circulation might therefore give rise to compromised tissue oxygenation. The unanticipated increase of $T C p_{\mathrm{O} 2}$ under resting conditions at stage $\mathrm{III}$, despite impaired nutritive skin blood flow, might result from facilitated oxygen diffusion through atrophic superficial skin layers as well as impaired oxygen consumption of these skin layers.

The observed increase in skin temperature at stage I as well as the decrease at stage III may reflect concomitant changes in thermoregulatory skin blood flow. The lower skin temperature, as observed at stage III, may have negatively influenced both nutritive and thermoregulatory skin blood flow. Considering the fact, however, that all measurements were performed in a temperature-controlled room under similar environmental conditions, it is conceivable that the lower skin temperature results from impaired thermoregulatory skin blood flow rather than inducing it.

Various scintigraphic studies revealed an increase of total extremity perfusion at stage 1, normalisation at stage II, and a decrease at stage III ${ }^{92,112,167}$. An important advantage of microcirculatory techniques over scintigraphic techniques is that the former are able to differentiate between the thermoregulatory and the nutritive part of the skin microcirculation.

In conclusion, this study indicates that 1.) thermoregulatory skin blood flow differs between RSD patient groups and controls as well as between the different stages of RSD, and 2.) there is a discrepancy in skin blood flow abnormalities between the thermoregulatory and the nutritive level of the skin microcirculation. Since the nutritive capillaries are likely to be controlled by local factors, whereas thermoregulatory skin blood vessels are predominantly sympathetically controlled, these findings may reflect sympathetic dysfunction. Moreover, this study is the first to report an increase of thermoregulatory skin blood flow in stage I RSD patients, which may be caused by a decrease in efferent sympathetic nerve impulses. The observed decrease in skin blood flow at stages II and III of the syndrome may reflect increased sensitivity of skin microvessels to (circulating) catecholamines. 


\section{Chapter 6}

\section{REFLEX SYMPATHETIC DYSTROPHY:}

\section{RESULT OF AUTONOMIC DENERVATION?}

HAJM Kurvers, MJHM Jacobs, RJ Beuk, FAJM van den Wildenberg, PJEHM Kitslaar, DW Slaaf, and RS Reneman

Departments of Surgery of the University Hospital Maastricht and of the Academic Medical Centre Amsterdam, The Netherlands.

Departments of Surgery, Biophysics and Physiology of the Cardiovascular Research Institute Maastricht (CARIM), The Netherlands.

Based on: Clinical Science, 1994, vol. 87, p. 663-669. 


\section{SUMMARY}

The exact nature of sympathetic dysfunction in RSD patients remains enigmatic. Mostly, sympathetic dysfunction has been purported to consist of an increased level of efferent sympathetic nerve impulses. According to this theory, any type of nerve or soft-tissue injury increases the level of sensory input to the spinal cord and thus the activity of the internuncial neuron pool. The latter may stimulate anterolateral sympathetic efferents, as a result of which the level of efferent sympathetic nerve impulses from the spinal cord to the affected extremity could become increased. However, the findings of some recent studies argue against the latter hypothesis. Consequently, we hypothesised that sympathetic dysfunction in RSD patients consists of supersensitivity to catecholamines consequent to (partial) sympathetic denervation. Microcirculatory vasoconstriction in limbs upon dependency is mediated by several mechanisms. At the thermoregulatory level of the skin microcirculation, the postural response is mainly sympathetically controlled, whereas at the nutritive level this response is considered to be controlled by non-neural factors. To investigate the nature of sympathetic dysfunction in the pathogenesis of RSD we, therefore, investigated these vasoconstrictive responses to dependency at both levels of the skin microcirculation. To this end, we performed laser Doppler flowmetry (LDF, in PU) and capillary microscopy (CM) in the skin of the hands of 76 RSD patients with unilateral disease. The patients were divided into 3 stages according to their perception of skin temperature (stage I in case of a stationary warmth sensation; stage II in case of an intermittent warmth and cold sensation, and stage III in case of a stationary cold sensation). The vasoconstrictor responses were induced by lowering of the affected hand. As compared to controls, the mainly sympathetically mediated vasoconstrictor response at the thermoregulatory level of the skin microcirculation, as measured by LDF, was attenuated at stage I, stage II, and stage III of RSD. As compared to thermoregulatory skin blood flow at heart level was found to be increased at stage I and decreased at stages II and III. The sympathetically-independent vasoconstrictor response at the nutritive level of the skin microcirculation, as measured by CM, was impaired only at stage III when compared with controls. Our findings of impaired vasoconstrictor responses to dependency at the thermoregulatory level of the skin microcirculation suggests (partial) sympathetic denervation of skin microvessels at all 3 clinical stages of RSD. This alleged sympathetic denervation may account for the observed increase in thermoregulatory skin blood flow at stage $\mathbb{I}$. Since sympathetic denervation has been shown to increase sensitivity of vascular structures to catecholamines, the decrease in thermoregulatory skin blood flow at stages II and III may result from microvascular supersensitivity to catecholamines.

\section{INTRODUCTION}

Reflex sympathetic dystrophy (RSD), which is usually localised in the extremities, is an incompletely understood pain syndrome. Generally, an increase in efferent sympathetic activity has been purported to play a pivotal role in the pathophysiology of the disorder $57,135,333,417$. 
Therefore, treatment of RSD has focussed predominantly on interruption of the alleged increase in efferent sympathetic nerve activity by means of sympathectomy, the outcome of which has not been invariably satisfactory ${ }^{370}$.

Microcirculatory vasoconstriction in limbs upon dependency is mediated by central and/or local (venoarteriolar or myogenic) mechanisms ${ }^{519}$. At the thermoregulatory level (arteriovenous anastomoses and subpapillary plexus), this postural response is predominantly controlled by a sympathetically mediated local venoarteriolar response ${ }^{519}$. At the nutritive level (capillaries), the vasoconstrictive response is considered to be mainly controlled by, sympathetically independent local factors, such as vasoactive metabolic substances accumulating during ischaemia and tissue oxygenation ${ }^{519,547}$.

To investigate the role of sympathetic dysfunction in the pathogenesis of RSD, we examined the postural response, by means of laser Doppler flowmetry (LDF) and capillary microscopy. LDF was used because arteriovenous anastomoses and the subpapillary plexus, which are richly innervated by sympathetic nerve endings ${ }^{219}$, contribute predominantly to the flow signal recorded with this technique ${ }^{153}$. Therefore, disturbances in thermoregulatory flow upon dependency, if any, may reflect sympathetic dysfunction. Capillary microscopy was used to study microcirculatory vasoconstrictive responses upon dependency at the nutritive level. The responses at this level were studied because they are likely to be controlled differently than the responses at the thermoregulatory level. If sympathetic dysfunction plays a role in the pathophysiology of RSD, vasoconstrictive responses upon dependency will be disturbed more at the thermoregulatory than at the nutritive level of skin microcirculation.

MATERIALS AND METHODS (for detailed information see chapter 4).

\section{Patients}

The study was performed on 76 patients with unilateral RSD of the upper extremity which all met the criteria described in chapter 4 . Events precipitating RSD were fractures (46\%), soft tissue traumas $(20 \%)$, operative procedures $(10 \%)$, neurovascular compressions $(10 \%)$, cerebrovascular accidents (1\%), and whiplash traumas (1\%). In $12 \%$ of the patients, no precipitating event could be traced. Smoking habits, age, and duration of disease are shown, per stage, in table 6.1. A group of 16 age-matched volunteers served as controls.

\begin{tabular}{|c|c|c|c|c|}
\hline STAGE & $\mathbf{N}$ & $\begin{array}{l}\text { SMOKERS } \\
(\%)\end{array}$ & $\begin{array}{l}\text { Age in years } \\
\text { mean (range) }\end{array}$ & $\begin{array}{l}\text { Duration of disease in months } \\
\text { (mean, range) }\end{array}$ \\
\hline Controls & 16 & 31 & $47(21-69)$ & - \\
\hline 1 & 15 & 60 & $50(24-74)$ & $2(1-7)$ \\
\hline III & 47 & 40 & $50(21-91)$ & $8(1-59)$ \\
\hline IIII & 14 & 57 & $43(16-65)$ & $40(2-120)$ \\
\hline
\end{tabular}

Table 6.1: patient characteristics: smoking habits, age, and duration of disease per group. 


\section{Experimental protocol}

Capillary microscopy was performed, with the hand at heart level, in the nailfold of the most symptomatic finger of RSD patients. Subsequently, arterial occlusion was induced by means of inflating a cuff around the upperarm. After acute deflation of the cuff capillary images were recorded during reactive hyperaemia. The measurements at heart level were followed by a five minute period during which the stage of the microscope was lowered thirty-five centimetres and the patient was allowed to adapt to the dependent position before the measurements were repeated. Subsequently, the affected extremity of the patient was placed on a pillow, again at heart level, for laser Doppler llowmetry and assessment of skin temperature. The laser Doppler probe holder was attached to the pulp of the most symptomatic finger. LDF measurements were performed at unheated skin to assess skin blood flow under physiological conditions. Simultaneously, skin temperature was measured at the dorsum of the same digit. LDF was assessed under resting conditions as well as during reactive hyperaemia, following release of arterial occlusion. Reactive hyperaemia was used to obtain an impression about the possible effect of large vessels, between the upperarm and hand; on microvascular reactivity ${ }^{519}$. Then the arm was lowered until the fingers were 35 centimetres below heart level. After stabilisation of blood flow, the foregoing measurements were repeated.

From the capillary microscopy recordings we obtained the red blood cell velocity under resting conditions ( $R B C V r, \mu \mathrm{m} / \mathrm{s})$ and at peak of reactive hyperaemia $(\mathrm{RBCVp}, \mu \mathrm{m} / \mathrm{s})$, both at heart level and in the dependent position. The individual heart level-to-dependent ratios for red blood cell velocity under resting conditions (R-RBCVr) and for peak red blood cell velocity (R-RBCVp) were calculated to evaluate the effectiveness of the sympathetically-independent postural vasoconstrictive mechanism at the nutritive level of the skin microcirculation. These ratios were defined as the velocities obtained at heart level divided by those obtained in the dependent position. A ratio lower than the one found in controls indicates an impaired constrictive mechanism. The lower this ratio is, the more this mechanism will be impaired. From the laser Doppler flow readings we obtained the mean flow under resting conditions (LDFr, PU) and the peak flow during reactive hyperaemia (LDFp, PU), both at heart level and in the dependent (dep) position. The individual heart level-to-dependent flow ratios for rest flow (R-LDFr) and for peak flow (R-LDFp) were calculated to evaluate the effectiveness of the sympathetically mediated postural vasoconstrictive mechanism at the thermoregulatory level of the skin microcirculation. These ratios were defined as the flow values obtained at heart level divided by those obtained in the dependent position. A ratio lower than the one found in controls indicates an impaired constrictive mechanism. The lower this ratio is, the more this mechanism will be impaired. Skin temperature (ST) was obtained at heart level under resting conditions.

\section{RESULTS (table 6.2)}

\section{Capillary microscopy}

As compared to healthy volunteers, red blood cell velocity at rest (RBCVr) was significantly decreased at stage II $(\mathrm{P}<0.01)$ and stage III $(\mathrm{P}<0.05)$, whereas maximum red blood cell velocity during reactive hyperaemia $(\mathrm{RBCV}$ ) was significantly reduced only at stage III ( $\mathrm{P}<$ 
$0.001)$. Changing from heart level to the dependent position resulted in a reduction of red blood cell velocity at rest (RBCVr) in controls, the ratio of llow velocity at heart level and in the dependent position being about 2.06. This ratio was unchanged in stage I patients, but decreased with progression of the syndrome $(\mathrm{P}=0.07$ at stage $\mathrm{I}, \mathrm{P}<0.05$ at stage III). Nutritive peak flow (RBCVp) slightly decreased with dependency in controls, resulting in a heart level-to-dependent ratio of about 1.18. This ratio was still unaffected at stage I and stage II, but was significantly lower in stage III patients ( $\mathrm{P}<0.05$ compared to controls, $\mathrm{P}=0.06$ compared to stage I). Linear regression analysis showed that both $\mathrm{R}-\mathrm{RBCVr}$ and $\mathrm{R}-\mathrm{RBCV} \mathrm{p}$ did not change as a function of duration of the syndrome. A consequence of the changes in the postural vasoconstrictive mechanisms is that the various parameters at dependency differ from those assessed at heart level. No significant differences were observed any more, between RSD patients on the one hand and controls on the other, in red blood cell velocity under resting conditions or at peak of reactive hyperaemia.

\begin{tabular}{|c|c|c|c|c|c|c|c|c|}
\hline & \multicolumn{2}{|l|}{ controls } & \multicolumn{2}{|l|}{ stage I } & \multicolumn{2}{|l|}{ stage II } & \multicolumn{2}{|c|}{ stage III } \\
\hline & HL & DEP & HL & DEP & HL & DEP & HL & DEP \\
\hline $\mathrm{RBCV} \mathbf{r}$ & $\begin{array}{l}318 \\
(231- \\
492)\end{array}$ & $\begin{array}{l}163 \\
(108- \\
270)\end{array}$ & $\begin{array}{l}322 \\
(159- \\
419)\end{array}$ & $\begin{array}{l}171 \\
(87- \\
224)\end{array}$ & $\begin{array}{l}168 \\
(89- \\
280)\end{array}$ & $\begin{array}{l}144 \\
(58- \\
243)\end{array}$ & $\begin{array}{l}204 \\
(65 \\
344)\end{array}$ & $\begin{array}{l}192 \\
(63- \\
259)\end{array}$ \\
\hline $\begin{array}{l}\text { R- } \\
\text { RBCV/r }\end{array}$ & \multicolumn{2}{|c|}{$2.06(1.18-2.85)$} & \multicolumn{2}{|c|}{$1.97(1.35-2.80)$} & \multicolumn{2}{|c|}{$1.15(0.86-2.20)$} & \multicolumn{2}{|c|}{$1.04(0.93-1.60)$} \\
\hline LDFr & $\begin{array}{l}81 \\
(51-109)\end{array}$ & $\begin{array}{l}39 \\
(9-49)\end{array}$ & $\begin{array}{l}152 \\
(80-195)\end{array}$ & $\begin{array}{l}77 \\
(49-153)\end{array}$ & $\begin{array}{l}54 \\
(36-79)\end{array}$ & $\begin{array}{l}58 \\
(32-9 \|)\end{array}$ & $\begin{array}{l}31 \\
(16-85)\end{array}$ & $\begin{array}{l}31 \\
(16-84)\end{array}$ \\
\hline $\begin{array}{l}\mathbb{R}- \\
\text { LDFr }\end{array}$ & \multicolumn{2}{|c|}{$1.82(1.41-7.69)$} & \multicolumn{2}{|c|}{$1.41(1.20-2.44)$} & \multicolumn{2}{|c|}{$1.09(0.82-1.39)$} & \multicolumn{2}{|c|}{$1.14(0.90-1.82)$} \\
\hline $\begin{array}{l}\text { Skin } \\
\text { Temp. }\end{array}$ & $\begin{array}{l}31.7 \\
(31.0 \\
33.1)\end{array}$ & $\begin{array}{l}32.2 \\
(31.4- \\
32.6)\end{array}$ & $\begin{array}{l}33.2 \\
(32.2- \\
33.9)\end{array}$ & $\begin{array}{l}32.6 \\
(32.1- \\
33.3)\end{array}$ & $\begin{array}{l}32.2 \\
(30.7- \\
33.1)\end{array}$ & $\begin{array}{l}31.9 \\
(30.4- \\
32.8)\end{array}$ & $\begin{array}{l}30.8 \\
(29.4- \\
32.5)\end{array}$ & $\begin{array}{l}30.6 \\
(29.8- \\
31.4)\end{array}$ \\
\hline
\end{tabular}

Table 6.2: data obtained by capillary microscopy, laser Doppler flowmetry and skin tempeirature measurement, obtained at heart level and in the dependent position, for both individual patient groups and controls. $H L=$ heart level, $D E P=$ dependent. $R B C V r=$ red blood cell velocity under resting conditions ( $\mu \mathrm{m} / \mathrm{s})$; $R-R B C V r=$ dependent to heart level ratio for $R B C V r$; $L D F r=$ mean flow under resting conditions (PU); $R$ $\mathrm{LDFr}=$ dependent to heart level ratio for $L D F r$; skin temp. $=$ skin temperature $\left({ }^{\circ} \mathrm{C}\right)$.

\section{Laser Doppler flowmetry}

As compared to controls, thermoregulatory skin blood flow at rest (LDFr, see fig. 1) evolved from an increase at stage I. of the syndrome $(\mathrm{P}<0.01)$ to a decrease at stage II $(\mathrm{P}<0.05)$ and III $(\mathrm{P}<0.05)$. A similar pattern was found for maximum skin blood flow during reactive hyperaemia (LDFp); as compared to healthy volunteers, it was significantly higher in stage I 
patients $(P<0.01)$ and tended to be lower in stage III patients $(P=0.08)$. In the control subjects LDF at rest (LDFr) decreased when the position of the hand was changed from heart level to the dependent position, resulting in a flow ratio of 1.82. As compared to controls the reduction of flow was far less at all stages of the syndrome (stage I, $\mathrm{P}<0.05$; stage II, $\mathrm{P}<$ 0.0001 ; stage III $P<0.01$ ). As compared to heart level, peak flow during reactive hyperaemia (LDFp) was also reduced in dependency resulting in a ratio of 1.3. Similar to rest flow this ratio was reduced with progression of the syndrome. As compared to healthy volunteers, the dependent to heart level peak LDF ratios (R-LDFp) were significantly lower in stage II ( $\mathrm{P}<$ $0.05)$ and stage III $(\mathrm{P}<0.05)$ RSD patients. Moreover, R-LDFp was significantly lower in stage II patients as compared to stage I patients $(\mathrm{P}<0.05)$ and in stage III patients as compared to stage II patients ( $\mathrm{P}<0.05$ ). Linear regression analysis revealed a marginal, but nevertheless statistically significant decrease in $\operatorname{R-LDFr}(\mathrm{Y}=1.1-(0.005 *$ duration of the syndrome); $95 \%$ $\mathrm{CI}-0.009$ to -0.001 ) as a function of duration of the syndrome (fig. 6.1). R-LDFp did not change as a function of duration of the syndrome. A consequence of the changes in the postural vasoconstrictive mechanisms is that the various parameters at dependency differ from those assessed at heart level. In the dependent position, thermoregulatory skin blood flow under resting conditions (LDFr), as compared to controls, remained significantly higher in stage I patients $(\mathrm{P}<0.01)$ and became significantly higher in stage II $(\mathrm{P}<0.05)$ patients. As compared to controls, maximum skin blood flow during reactive hyperaemia (LDFp) remained significantly higher in stage I RSD patients $(\mathrm{P}<0.05)$.

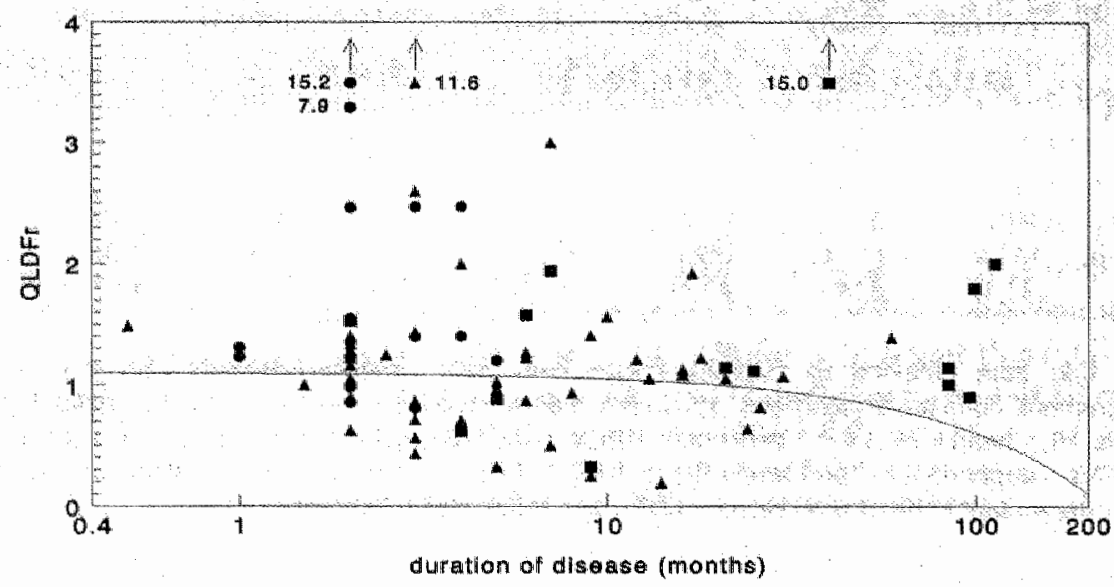

Flg. 6. 1 : relationship between the heart level-to-dependent flow ratios for rest flow (R-LDFr) and duration of the RSD syndrone yideled the following linear regression equation $Y=1.1-(0.005 \times X), 95 \% \mathrm{Cl}$ slope -0.009 to -0.001 on the $x$-axis is plotted the duration of the RSD syndrome in months (logarithmic scale), and on the y axis the ratio of total skin blood flow at rest in dependent to heart lewel - indicates stage I patients; 4 indicates stage 11 patients; stage lll patients: 


\section{Skin temperature}

As compared to controls, skin temperature at heart level was significantly higher at stage I ( $P$ <0.01) and tended to be lower at stage III $(\mathrm{P}=0.08)$.

\section{DISCUSSION}

The findings of the present study indicate that, at the themoregulatory level of skin microcirculation, the sympathetically mediated vasoconstrictive response to dependency is disturbed at all stages of RSD. Moreover, the disturbance in vasoconstriction progresses with both clinical stage and duration of the syndrome. At the nutritive level of skin microcirculation vasoconstriction on dependency was impaired only at stage III.

The vasoconstrictive response to dependency at the thermoregulatory level of skin microcirculation is mainly controlled by a sympathetically mediated venoarteriolar response of local origin ${ }^{220,223,519}$. Since the postural vasoconstriction is abolished after local nervous blockade by lidocaine ${ }^{219}$ as well as after sympathectomy ${ }^{219}$, failure of this response, as observed at all stages of the RSD syndrome, may indicate autonomic (sympathetic) denervation. The impaired vasoconstrictive response at stages II and III has been purported to result from an already increased efferent sympathetic nerve activity in the resting state ${ }^{441}$. In our study however, the vasoconstrictive response was also disturbed in patients with increased thermoregulatory skin blood flow at heart level (stage I), indicating a dlecrease rather than an increase in efferent sympathetic nerve activity. Hence, the alleged autonomic (sympathetic) denervation may account for the increase in thermoregulatory skin blood flow at heart level as observed at stage I. Since sympathetic denervation has been reported to cause increased sensitivity of vascular structures to catecholamines 42,233 , the observed decrease in thermoregulatory blood flow as measured at heart level at stages II and III may be explained by hypersensitivity to catecholamines of skin microvessels. The hypothesis of sympathetic denervation is supported by a study of Drummond and colleagues ${ }^{138}$ who observed a decrease in venous plasma levels of noradrenaline, collected in the affected limb of RSD patients. Moreover, Arnold and colleagues ${ }^{19}$ presented direct in-vivo evidence for hypersensitivity of venous alpha-adrenoceptors to noradrenaline in limbs affected by RSD.

As alluded to before, constriction of thermoregulatory skin microvessels consequent to dependency of an extremity is predominantly controlled by a sympathetically mediated veno arteriolar response of local origin ${ }^{220,223}$ and to a minor extent by a local myogenic response ${ }^{220,519}$. Hence, it can not be excluded that myogenic dysfunction is involved in the impairment of this vasoconstrictive response as observed at all stages of the RSD syndrome. However, the finding in our study that the vasoconstrictive response to dependency was undisturbed at the nonsympathetically controlled ${ }^{547}$ nutritive level of skin microcirculation at stages I and II of the RSD syndrome is in favour of the hypothesis of sympathetic dysfunction.

At the nutritive level of skin microcirculation, the vasoconstrictive response to dependency is considered to be controlled by local factors, such as vasoactive metabolic

substances accumulating during ischaemia and tissue oxygenation ${ }^{519,547}$. The observed impairment of the vasoconstrictive response at the nutritive level of skin microcirculation at 
stage III of the RSD syndrome therefore may be caused by locally acting vasoactive metabolites resulting from longstanding impairment of the nutritive skin blood flow.

A limitation of the methodological approach used in our study is that it provides an indirect measure of sympathetic tone. The most direct way to study efferent sympathetic nerve activity would be micro-electrode recordings. These are however difficult to calibrate ${ }^{138}$ because the signal is changed by slight adjustments to the position of the electrode tip. In addition, positioning the electrode correctly is technically difficult and may be painful, possibly influencing efferent sympathetic nerve activity through a somato-sympathetic reflex.

The venoarteriolar response on dependency acts as an important oedema prevention mechanism. It limits the expected rise in capillary hydrostatic pressure ${ }^{220}$ and allows a build-up of capillary oncotic pressure ${ }^{389}$. Failure of this response, as observed at all stages of RSD, will expose the skin microvascular bed to raised capillary pressures. This increases in capillary pressure in combination with an increase in thermoregulatory skin blood flow, as observed at heart level at stage I and in the dependent position at both stages I and II, may contribute to oedema formation. The latter is observed at all stages of the RSD syndrome, but most frequently at stages I and II, which is in line with our microcirculatory findings. Moreover, raised capillary pressures may promote thickening of capillary basement membranes ${ }^{427}$, which has been reported to occur in RSD patients ${ }^{385}$. Ultimately, these structural changes of capillary basement membranes may contribute to impairment of nutrition of the skin, resulting in trophic changes, which develop frequently in patients with longstanding RSD. Tissue hypersensitivity to catecholamines may also explain the deterioration of complaints, and the occurrence of vasoconstriction in response to stimuli known to provoke an increase in circulating catecholamines, such as emotion and cold.

So far, most hypotheses in the literature concerning involvement of the sympathetic nervous system postulate that an increase in efferent sympathetic nerve activity, through a somato-sympathetic reflex, plays a pivotal role within the pathophysiological mechanisms underlying RSD ${ }^{57,135,333,417}$. Hence, treatment of RSD has mainly focused on blocking of the alleged increase in efferent sympathetic nerve activity by means of sympathectomy, the results of which have not been invariably satisfactory ${ }^{370}$. If, however, sympathetic dysfunction mainly results from increased sensitivity to catecholamines consequent to sympathetic denervation, blocking of adrenoceptors might yield interesting therapeutical perspectives with respect to the sympathetically maintained complaints. The latter is supported by reported beneficial effects of intravenous phentolamine ${ }^{17}$ and oral phenoxybenzamine ${ }^{180}$ in patients with RSD. 


\section{Chapter 7}

REFLEX SYMPATHETIC DYSTROPHY:

THE INFLUENCE OF LOCAL SKIN HEATING AND REACTIVE HYPERAEMIA ON SKIN BLOOD FLOW ABNORMALITIES

HAJM Kurvers, MJHM Jacobs, RJ Beuk, FAJM van den Wildenberg, PJEHM Kitslaar, DW Slaaf, and RS Reneman

Departments of Surgery of the Academic Hospital Maastricht and of the Academic Medical Centre Amsterdam, The Netherlands.

Departments of Surgery, Biophysics and Physiology of the Cardiovascular Research Institute Maastricht (CARIM), The Netherlands.

Based on: European Journal of Clinical Investigation, 1995, vol. 25, p. 346-352. 


\section{SUMMARY}

Skin blood flow in reflex sympathetic dystrophy (RSD) patients has been reported to develor from an increase at an early stage to a decrease at later stages. Sofar, it remains unclear whethe these abnormalities are solely of microcirculatory origin, and result from functional vasospasm or structural vessel wall changes. Eighty-seven RSD patients were categorised as follows: stage I in case of a stationary warmth sensation, stage II in case of an intermittent warmth and cold sensation, and stage III in case of a stationary cold sensation. Laser Doppler flowmetry (LDF) was used as a measure of total skin blood flow and transeutaneous oximetry $\left(\mathrm{TC} p_{\mathrm{O} 2}\right)$ as a measure of vascular reactivity in the more superficial skin layers. Local skin heating and reactive hyperaemia were used to study the relative reserve capacity of skin microvessels, Finapres was used to assess digital arterial pressures. As compared to healthy volunteers $(\mathbb{N}=$ 16), LDF under control conditions demonstrated an increase in skin blood flow at stage I. A decrease in skin blood flow under control conditions was seen at stages II and III, but the relative flow reserve capacity, as measured with LDF, was not impaired at these stages. Regression analysis did not show a relation between LDF parameters and duration of the syndrome. $T C p_{\mathrm{O} 2}$ revealed no differences between patient groups and controls. Regression analysis did not demonstrate a relation between $T C p_{\mathrm{O}_{2}}$ parameters and duration of the syndrome. Digital systolic blood pressures were increased at stages II and III. These findings indicate that abnormalities in skin blood flow, as observed in RSD patients are of microcirculatory origin. The observed decrease in skin blood flow at stages II and III most likely results from functional vasospasm rather than from structural vessel wall changes.

\section{INTRODUCTION}

Reflex sympathetic dystrophy (RSD) is a pain syndrome which is characterised by motor, sensory, and sympathetic disturbances. The latter is reflected, among others, by abnormalities in skin blood flow within the affected extremity, which has been reported to be increased at an early stage and to be decreased at later stages of the syndrome ${ }^{299}$. The observed decrease at later stages has been associated with an increase in efferent sympathetic nerve activity $57,135,417$. The findings in some recent studies, however, indicate that both sympathetic denervation ${ }^{138,298}$ and hypersensitivity to catecholamines ${ }^{19}$ may play a role in RSD.

Sofar, it remains unclear whether abnormalities in skin blood flow, as observed in RSD, are solely of microcirculatory origin. Alternatively, impairment of the macrocirculation may play a role. Additionally, it is unknown whether these skin blood flow abnormalities can be attributed to functional vasospasm and/or structural changes of the vessel wall.

Therefore, we investigated skin microcirculation in the three clinical stages of RSD, using laser Doppler flowmetry and transcutaneous oximetry. Measurements were performed before and after arterial occlusion, and at different local skin temperatures. Arterial occlusion induces a reactive hyperaemic response, whereas local skin heating overrules local vasoconstrictive mechanisms. Both provocative techniques provide an impression about the possible role of larger vessels, between the upperarm and hand, on microvascular disturbances, if any $y^{519,521}$. 
Additionally, by comparing the microcirculatory values, as assessed during local skin heating: and postocclusive reactive hyperaemia, with the preheating and pre-occlusive microcirculatory values, a relative measure for the vasodilator capacity of skin microvessels can be obtained ${ }^{422}$, Wollersheim and colleagues ${ }^{561}$ demonstrated that this vasodilator capacity of skin microvessels is impaired in the case of structural vessel wall changes, whereas it is unchanged in the case of purely functional vasospastic disorders.

We opted for laser Doppler flowmetry because this investigative technique has been reported to provide information predominantly on the sympathetically controlled thermoregulatory part of skin microcirculation ${ }^{153}$. Transcutaneous oximetry was used to assess vascular reactivity in more superficial skin layers ${ }^{95,305}$. Digital arterial blood pressure was determined, as a measure of the macrocirculation, by means of the Finapres volume-clamp technique ${ }^{245}$.

PATIENTS AND METHODS (for detailed informatiuon, see chapter 4)

\section{Patients}

Eighty-seven RSD patients, with unilateral complaints, met the standards described in detail in chapter 4 . The age of the 24 males and 52 females ranged from 16 to 91 years, with a mean value of 49 years. Events precipitating RSD were fractures ( $45 \%$ ), soft tissue traumas ( $20 \%)$, operative procedures $(10 \%)$, neurovascular compressions $(10 \%)$, cerebrovascular accidents $(1 \%)$, and whiplash traumas (1\%). In $11 \%$ of the patients, no precipitating event could be traced. Smoking habits, age, and duration of disease are shown, per stage, in table 7.1. A group of 16 age-matched volunteers was investigated as controls.

\begin{tabular}{lllll}
\hline STAGE & N & $\begin{array}{l}\text { SM } \\
(\%)\end{array}$ & $\begin{array}{l}\text { MEAN AGE } \\
\text { (years, range) }\end{array}$ & $\begin{array}{l}\text { MEAN DURATION OF DISEASE } \\
\text { (months, range) }\end{array}$ \\
\hline Con & 16 & 31 & $47(21-69)$ & - \\
I & 20 & 60 & $50(24-74)$ & $2(1-7)$ \\
II & 47 & 40 & $50(21-91)$ & $8(1-59)$ \\
III & 20 & 60 & $43(16-65)$ & $36(2-120)$ \\
\hline
\end{tabular}

Table 7.1: patient characteristics: per stage absolute number $(N)$, percentage smokers (SM), age (mean and range), and duration of syndrome (mean and range).

\section{Experimental protocol}

$\mathrm{LDF}$ and $\mathrm{TC} p_{\mathrm{O} 2}$ were assessed under resting conditions, during arterial occlusion, as well as during the subsequent reactive hyperaemia. During the first part of the investigations, LDF 
measurements were performed at unheated skin in order to assess skin blood flow under physiological conditions. TCp $\mathrm{O}_{2}$ measurements were performed at a probe temperature of 37 ${ }^{\circ} \mathrm{C}$, resulting in incomplete suppression of local vasoconstrictive mechanisms. In the second part of the investigation, LDF was also performed during incomplete suppression of local vasoconstriction, at a probe temperature of $40^{\circ} \mathrm{C}$. Simultaneously, $\mathrm{TC} p_{\mathrm{O} 2}$ measurements were performed during complete suppression of Jocal vasoconstrictive mechanisms, at a probe temperature of $44^{\circ} \mathrm{C}^{52 \%}$.

From the LDF recordings (obtained at local skin temperature and $40^{\circ} \mathrm{C}$ ) and $T C p_{\mathrm{O} 2}$ recordings (obtained at $37^{\circ} \mathrm{C}$ and $44^{\circ} \mathrm{C}$ ), we assessed: 1 ) the mean values under resting conditions (LDFr in $\mathrm{PU}, \mathrm{TC} \mathrm{P}_{2} \mathrm{r}$ in $\mathrm{mm} \mathrm{Hg}$ ), 2) the peak values during reactive hyperaemia (LDFp in PU, TC $p_{\mathrm{O} 2} \mathrm{p}$ in mm Hg), and 3) the time to reach peak values (LDFt in $\mathrm{s}, \mathrm{TC} p_{\mathrm{O}_{2} \mathrm{t}}$ in s). Biological zero flow was subtracted from the resting and peak LDF flow values measured. Since submaximal hyperaemic responses depend on the pre-occlusive or preheating microcirculatory values ${ }^{109}$, it is not appropriate to compare the values obtained during reactive hyperaemia and/or local skin heating between patient groups and controls. We therefore compared the relative microcirculatory values, being defined as the post provocative value divided by the preprovocative one, as a measure of the relative reserve capacity of skin microvessels. These relative values are calculated as follows. The relative values during postocclusive reactive hyperaemia: R-LDF hyp defined as LDFp at unheated skin divided by $\mathrm{LDFr}$ at unheated skin and R-TC $p_{\mathrm{O} \text { athy }}$ defined as TC $p_{\mathrm{O} 2} \mathrm{p}$ at probe temperature of $37^{\circ} \mathrm{C}$ divided by $\mathrm{TC} p_{O 2} \mathrm{r}$ at probe temperature of $37^{\circ} \mathrm{C}$. The relative values during local skin heating: $\mathrm{R}$ $L D F$ hea defined as LDFr at probe temperature of $40^{\circ} \mathrm{C}$ divided by LDFr at unheated skin and $\mathrm{R}-\mathrm{TC} \mathrm{P}_{\mathrm{O} 2 \mathrm{hea}}$ defined as $\mathrm{TC} \mathrm{P}_{\mathrm{O}_{2} \mathrm{r}} \mathrm{r}$ at probe temperature of $44^{\circ} \mathrm{C}$ divided by $\mathrm{TC} \mathrm{p}_{\mathrm{O}_{2} \mathrm{r}} \mathrm{r}$ at probe temperature of $37^{\circ} \mathrm{C}$. A relative value smaller than the one found in controls indicates impaired vasodilator capacity and thus very likely structural vessel wall changes. From the finapres recordings we obtained systolic (SBP), diastolic (DBP) and mean (MBP) arterial blood pressure ( $\mathrm{mm} \mathrm{Hg}$ ).

\section{Statistics}

The statistics used have been described in detail in chapter 4. Additionally, the non-parametric Kruskall-Wallis test was used as an overall test of differences between the four groups (controls and three patient groups). Bonferrroni correction factor was employed to control for type I error due to multiple comparisons.

\section{RESULTS}

\section{Laser Doppler flowmetry (table 7.2)}

Resting skin blood flow (LDFr) at unheated skin differed $(p<0.0001)$ between the four groups. As compared to controls, LDFr at unheated skin was increased at stage $\mathrm{I}(\mathrm{p}<0.01)$ and decreased at stages II $(p<0.05)$ and III $(p<0.01)$. No differences in the time-to-peak flow were found between the controls and the three patients groups. Linear regression analysis revealed no relation between resting skin blood flow at unheated skin and duration of the syndrome $(\mathrm{Y}=85-(0.21 *$ duration of syndrome $) ; 95 \% \mathrm{Cl}=-0.51$ to $0.1 ; \mathrm{R}=-0.20)$. The 
relative flow reserve capacity, as tested by postocclusive reactive hyperaemia (R-LDF typp $_{2}$ ) differed $(p<0.001)$ between the four groups. As compared to controls, R-LDF hyp did not differ at stages I and II, and was increased at stage III $(p<0.01)$. The relative flow reserve capacity, as tested by local skin heating $(R-L D F$ hea $)$ also differed $(p<0.0001)$ between the four groups. As compared to controls, $R-L D F$ hea was decreased $(p<0.01)$ at stage $I$, did not differ at stage II, and was increased at stage III $(p<0.05)$. Linear regression analysis showed no relation between duration of the syndrome on the one hand and 1$) \mathrm{R}-\mathrm{LDF}$ hyp $(\mathrm{Y}=3+(0.0 \mathrm{I} *$ duration of syndrome); $95 \% \mathrm{Cl}=-0.05$ to $0.06 ; \mathrm{R}=0.18)$ and 2$) \mathrm{R}-\mathrm{LDF} \mathrm{Hei}_{\text {hei }}(\mathrm{Y}=4.6+(0.06 *$ duration of syndrome); $95 \% \mathrm{CI}=-0.06$ to $0.19 ; \mathrm{R}=0.23$ ) on the other.

\begin{tabular}{|c|c|c|c|c|c|c|c|c|}
\hline & \multicolumn{2}{|c|}{ controls } & \multicolumn{2}{|c|}{ stage I } & \multicolumn{2}{|c|}{ stage II } & \multicolumn{2}{|c|}{ stage IIII } \\
\hline & LST & $40^{\circ} \mathrm{C}$ & LST & $40^{\circ} \mathrm{C}$ & LST & $40^{\circ} \mathrm{C}$ & LST & $40^{\circ} \mathrm{C}$ \\
\hline (LDFr) & $\begin{array}{l}81 \\
(51- \\
109)\end{array}$ & $\begin{array}{l}170 \\
(134- \\
192)\end{array}$ & $\begin{array}{l}152 \\
(91- \\
193)\end{array}$ & $\begin{array}{l}179 \\
(135 \\
210)\end{array}$ & $\begin{array}{l}54 \\
(36- \\
79)\end{array}$ & $\begin{array}{l}133 \\
(104- \\
183)\end{array}$ & $\begin{array}{l}30 \\
(15- \\
72)\end{array}$ & $\begin{array}{l}137 \\
(115- \\
170)\end{array}$ \\
\hline (LDFp) & $\begin{array}{l}125 \\
(79- \\
148)\end{array}$ & $\begin{array}{l}222 \\
(193- \\
2511)\end{array}$ & $\begin{array}{l}202 \\
(141- \\
230)\end{array}$ & $\begin{array}{l}199 \\
(141- \\
243)\end{array}$ & $\begin{array}{l}99 \\
(64- \\
134)\end{array}$ & $\begin{array}{l}200 \\
(116- \\
245)\end{array}$ & $\begin{array}{l}82 \\
(53- \\
134)\end{array}$ & $\begin{array}{l}164 \\
(139- \\
206)\end{array}$ \\
\hline (LDFt) & $\begin{array}{l}24 \\
(20- \\
32)\end{array}$ & $\begin{array}{l}30 \\
(24- \\
37)\end{array}$ & $\begin{array}{l}21 \\
(14- \\
30)\end{array}$ & $\begin{array}{l}28 \\
(20- \\
36)\end{array}$ & $\begin{array}{l}25 \\
(13- \\
31)\end{array}$ & $\begin{array}{l}31 \\
(24- \\
38)\end{array}$ & $\begin{array}{l}28 \\
(19- \\
40)\end{array}$ & $\begin{array}{l}36 \\
(27- \\
48)\end{array}$ \\
\hline$\left(R-L_{\text {hyp }}\right)$ & \multicolumn{2}{|c|}{$1.42(1.28-1.61)$} & \multicolumn{2}{|c|}{$1.25(1.16-1.47)$} & \multicolumn{2}{|c|}{$1.61(1.27-2.16)$} & \multicolumn{2}{|c|}{$2.43(1.72-3.7)$} \\
\hline$\left(R-L^{-} F_{\text {heal }}\right)$ & \multicolumn{2}{|c|}{$1.97(1.8-2.68)$} & \multicolumn{2}{|c|}{$1.14(1.02-1.60)$} & \multicolumn{2}{|c|}{$2.19(1.8-3.69)$} & \multicolumn{2}{|c|}{$3.99(2.32-7.67)$} \\
\hline
\end{tabular}

Tablle 7.2: laser Doppler flowmetry data, patient groups and controls, medians and interquartile ranges, at local skin temperature (LST) and at probe temperature of $40^{\circ} \mathrm{C}$. LDFr: skin blood flow under resting conditions; LDFp: peak skin blood flow; LDFt: time to peak skin blood flow: R-LDF inp: relative increase postocclusive hyperaemia; $R-L D F$ : relative increase local skin heating.

\section{Transcutaneous oximetry (table 7.3)}

Oxygen tension under resting conditions, peak oxygen tension during reactive hyperaemia, and the time to reach peak oxygen tension did not differ between the four groups. Linear regression analysis revealed no relation between duration of the syndrome and oxygen tension under resting conditions ( $\mathrm{Y}=64-(0.02 *$ duration of syndrome); $95 \% \mathrm{Cl}=-0.09$ to $0.05 ; \mathrm{R}=$ $0.03)$. The relative microcirculatory reserve capacity, as tested by postocclusive reactive hyperaemia $\left(\mathbb{R}-\mathrm{TC} p_{\text {Ozhyp }}\right)$ and local skin heating $\left(\mathrm{R}-\mathrm{TC} p_{\text {ozhea }}\right.$ ) did not differ between the four groups. Linear regression analysis showed no relation between duration of the syndrome and 1) $\mathrm{R}-\mathrm{TC} p_{\mathrm{O} 2 \mathrm{hyp}}(\mathrm{Y}=3.8+(0.004 *$ duration of syndrome $) ; 95 \% \mathrm{CI}=-0.02$ to $0.02 ; \mathrm{R}=0.07)$, and 2) $\mathrm{R}-\mathrm{TC} p_{\text {O2hea }}(\mathrm{Y}=15.1-(0.01 *$ duration of syndrome $) ; 95 \% \mathrm{CI}=-0.08$ to $0.06 ; \mathrm{R}=-$ $0.03)$, respectively. 


\begin{tabular}{|c|c|c|c|c|c|c|c|c|}
\hline & \multicolumn{2}{|c|}{ cointrols } & \multicolumn{2}{|l|}{ stagel } & \multicolumn{2}{|c|}{ stage II } & \multicolumn{2}{|c|}{ stage III } \\
\hline & $37^{\circ} \mathrm{C}$ & $44^{\circ} \mathrm{C}$ & $37^{\circ} \mathrm{C}$ & $44^{\circ} \mathrm{C}$ & $37^{\circ} \mathrm{C}$ & $44^{\circ} \mathrm{C}$ & $37^{\circ} \mathrm{C}$ & $44^{\circ} \mathrm{C}$ \\
\hline$\left(\mathrm{TC} p_{c /}\right)$ & 7 & $\begin{array}{l}58 \\
(49-75)\end{array}$ & $\begin{array}{l}5 \\
(4-7)\end{array}$ & $\begin{array}{l}65 \\
(57-74)\end{array}$ & $\begin{array}{l}5 \\
(3-8)\end{array}$ & $\begin{array}{l}65 \\
(55-71)\end{array}$ & $\begin{array}{l}5 \\
(3-8)\end{array}$ & $\begin{array}{l}(54 \\
79)\end{array}$ \\
\hline$\left(T C p_{022} p\right)$ & $\begin{array}{l}18 \\
(12- \\
20)\end{array}$ & $\begin{array}{l}66 \\
(55-78)\end{array}$ & $\begin{array}{l}18 \\
(13-20)\end{array}$ & $\begin{array}{l}72 \\
(63-77)\end{array}$ & $\begin{array}{l}16 \\
(13-22)\end{array}$ & $\begin{array}{l}66 \\
(60-77)\end{array}$ & $\begin{array}{l}18 \\
(13-24)\end{array}$ & $\begin{array}{l}69 \\
(61- \\
75)\end{array}$ \\
\hline$\left(T C p_{02} t\right)$ & $\begin{array}{l}82 \\
(58- \\
103)\end{array}$ & $\begin{array}{l}131 \\
(122 \\
147)\end{array}$ & $\begin{array}{l}81 \\
(67-97)\end{array}$ & $\begin{array}{l}137 \\
(123- \\
157)\end{array}$ & $\begin{array}{l}75 \\
(59-83)\end{array}$ & $\begin{array}{l}141 \\
(122 \\
168)\end{array}$ & $\begin{array}{l}83 \\
(73- \\
102)\end{array}$ & $\begin{array}{l}164 \\
(138- \\
197)\end{array}$ \\
\hline & & & & & $\because$ & & & \\
\hline$\left(R-T C p_{\text {ozhyps }}\right)$ & \multicolumn{2}{|c|}{$1.1(1.06-1.17)$} & \multicolumn{2}{|c|}{$1.08(1.03-1.13)$} & \multicolumn{2}{|c|}{$1.05(1-1.12)$} & \multicolumn{2}{|c|}{$1.07(0.97-1.15)$} \\
\hline$\left(\mathrm{R}-\mathrm{TC} \mathrm{p}_{\mathrm{Ozha \textrm {a }}}\right)$ & \multicolumn{2}{|c|}{$3.88(3.05-4.86)$} & \multicolumn{2}{|c|}{$4.11(3.51-4.67)$} & \multicolumn{2}{|c|}{$4.15(3.27-5.7)$} & \multicolumn{2}{|c|}{$3.82(3.13-4.44)$} \\
\hline
\end{tabular}

Table 7.3: transcutaneous oximetry data, patient groups and controls, medians and interquartile ranges ${ }_{1}$ at probe temperatures of $37^{\circ} \mathrm{C}$ and $44^{\circ} \mathrm{C}$. Tcp ${ }_{02} r$ oxygen tension under resting conditions; $T c p{ }_{o 2} \mathrm{p}$ : peak oxygen tension; TCP ${ }_{22}$ t time to peak oxygen tension; $R-T c p_{\text {ozhyp }}$; relative increase postocelusive hyperaemia; $R-$ $T C p_{\text {ozhas: }}$ relative increase local skin heating.

Finapres (table 7.4)

Systolic blood pressure (SBP, $<<0.01$ ), diastolic blood pressure (DBP, $p<0.01$ ), and mean blood pressure (MBP, $p<0.001$ ) differed between the four groups. As compared to controls, 1) SBP was higher at stages II ( $p<0.05)$ and III $(p<0.001)$, 2) DBP was higher at stage III ( $p$ $<0.01$ ), and 3) MBP was higher at stage III ( $p<0.001)$.

\begin{tabular}{lllll}
\hline & controls & stage I & stage II & stage III \\
\hline $\begin{array}{l}\text { Systolic blood } \\
\text { pressure }\end{array}$ & 116 & 128 & 130 & 140 \\
Diastolic blood & $(103-130)$ & $(106-137)$ & $(116-147)$ & $(137-143)$ \\
pressure & 67 & 74 & 75 & 84 \\
Mean blood & $(61-80)$ & $(61-85)$ & $(67-81)$ & $(78-88)$ \\
pressure & 82 & 90 & 91 & 103 \\
\hline
\end{tabular}

Table 7.4: Finapres data, patient groups and controls, medians and interquartile ranges. 


\section{DISCUSSION}

As compared to healthy controls, LDF indicates that skin blood flow is increased at stage I. A decrease in skin blood flow was observed at stages II and III, but the relative flow reserve capacity at these stages was not impaired. The observed disturbances are likely to occur at the microcirculatory level, because the time-to-peak skin blood flow, which is considered to provide information predominantly about the larger vessells ${ }^{519}$, did not differ between patient groups and controls. The hypothesis that, in RSD patients the abnormalities in skin blood flow are of microcirculatory origin is in line with the observation that digital arterial pressures were not impaired at any stage of the syndrome. The finding that in RSD patients skin blood flow abnormalities are of microcirculatory origin supports the hypothesis that sympathetic dysfunction is a component of the pathophysiological mechanisms underlying RSD, since skin microvessels are more sensitive to vasoconstrictor effects of catecholamines than larger vessels $^{556}$.

Skin blood flow was impaired at stages II and III of the RSD syndrome, indicating vasoconstriction, which may result from functional vasospasm and/or structural vessel wall changes $^{561}$. Our finding that at these stages the relative flow reserve capacity of skin microvessels was not impaired indicates that the observed decrease in skin blood flow results from functional vasospasm rather than from structural vessel wall changes. This hypothesis is in line with the finding that at a probe temperature of $44^{\circ} \mathrm{C}$, which has been reported to overrule completely local vasoconstrictive mechanisms ${ }^{343}$, transcutaneous oximetry revealed no differences between RSD patients and controls. The observed impairment of the relative flow reserve capacity at stage $\mathrm{I}$, as tested by local skin heating, most likely results from increased preheating skin blood flow values at this stage. The increase in relative flow reserve capacity at stage III, as tested by postocclusive reactive hyperaemia, is likely to result from decreased preheating skin blood flow values at this stage.

Regression analysis revealed no relation between resting skin blood flow at unheated skin and duration of the syndrome. In contrast, resting skin blood flow at unheated skin was increased at stage I and decreased at stages II and III, both as compared to controls. This finding demonstrates that in RSD patients changes in skin blood flow correspond better with stage than with duration of the syndrome, suggesting that there is a large interindividual variance in rapidity of onset of pathophysiological mechanisms causing the skin blood flow abnormalities.

Hypersensitivity to catecholamines, resulting from sympathetic denervation, may involve not only skin microvessels but also arterial resistance vessels. Hence, the observed increase in systolic blood pressures at stages II and III may have resulted from hypersensitivity-induced constriction of these arterial resistance vessels.

In conciusion, the results of the present study demonstrate that skin blood flow abnormalities as observed in RSD patients are of microcirculatory origin and result from functional vasospasm rather than from structural vessel wall changes. 


\section{Chapter 8}

\section{REFLEX SYMPATHETIC DYSTROPHY:}

\section{THE SPINAL COMPONENT}

TO SKIN BLOOD FLOW ABNORMALITIES

HAJM Kurvers, MJHM Jacobs, RJ Beuk, FAJM van den Wildenberg, PJEHM Kitslaar, DW Slaaf, and RS Reneman

Departments of Surgery of the University Hospital Maastricht and of the Academic Medical Centre Amsterdam, The Netherlands.

Departments of Biophysics, Physiology, and Surgery of the Cardiovascular

Research Institute Maastricht (CARIM), The Netherlands. 


\section{SUMMARY}

Reflex Sympathetic Dystrophy (RSD) is a neuropathic pain syndrome, characterised amongst others by skin blood flow abnormalities, which have been associated with sympathetic vasoconstrictor and antidromic vasodilator mechanisms. We investigated whether these mechanisms 1) are solely of peripheral origin or additionally have a spinal component, and 2) act exclusively through neural or involve also humoral pathways. 54 RSD patients with unilateral disease were divided into three stages according to their perception of skin temperature in the clinically affected hand (stage I: stationary warmth sensation; stage II intermittent warmth and cold sensation; stage III stationary cold sensation). Investigation of basal skin blood flow and vasoconstrictor response to dependency of skin microvessels in the clinically unaffected hand (CUH) of RSD patients as well as in the left hand of 16 controls (CON). The microcirculation was investigated at the predominantly neurally controlled thermoregulatory level (laser Doppler flowmetry) as well as at the predominantly humorally controlled nutritive level (capillary microscopy). In the CUH, at thermoregulatory level of the skin microcirculation: 1) basal SBF was increased at stage I as compared to the CON. The vasoconstrictor response to dependency (defined as SBF at heart level divided by SBF in the dependent position) was attenuated at stage I as compared to the CON, 3) basal SBF and the vasoconstrictor response to dependency did not differ from the CON at stages II and III. In the $\mathrm{CUH}$, at nutritive level, no differences could be observed at any stage of the syndrome when compared with the CON. This study indicates that there is a spinal component to microcirculatory abnormalities at stage I of the RSD syndrome, which most likely acts through neural (antidromic vasodilator) mechanisms, and which may be initiated by (post-) traumatic excitation of a peripheral nerve on the clinically affected side.

\section{INTRODUCTION}

It has been demonstrated that some patients with reflex sympathetic dystrophy (RSD) develop minor signs and symptoms on the clinically unaffected side ("mirror imaging") ${ }^{294,463}$. This suggests that there is a spinal component to the pathophysiological mechanisms underlying this neuropathic pain syndrome.

In previous studies, we demonstrated that total skin blood flow in the clinically affected hand develops from an increase at stage I to a decrease at stages II and III ${ }^{299,300}$. These abnormalities in skin blood flow have been associated with antidromic as well as sympathetic mechanisms ${ }^{460}$. Both antidromic vasodilatation and sympathetic dysfunction act through neural mechanisms, which may be not only of peripheral, but also of spinal origin. If the disturbances would be exclusively of peripheral origin, skin blood flow abnormalities should only be present in the clinically affected hand. Alternatively, if spinal mechanisms would be involved, skin blood flow may additionally be altered in the clinically unaffected hand. To differentiate between spinal and peripheral mechanisms, we examined basal skin blood flow as well as the vasoconstrictor response to dependency of skin microvessels in the clinically unaffected hand of RSD patients. 
Skin blood flow abnormalities in the clinically unaffected hand of RSD patients, if any, may be not only of neural, but also of humoral origin. Therefore, we assessed skin microcirculation both at the thermoregulatory and at the nutritive level. Laser Doppler flowmetry (LDF) was used to study skin blood flow at the thermoregulatory level because arteriovenous anastomoses and the subpapillary plexus, which are richly innervated ${ }^{219,547}$, contribute predominantly to the flow signal recorded with this technique ${ }^{533}$. Ergo, disturbances in thermoregulatory flow may reflect neural dysfunction. Capillary microscopy was used to assess the nutritive part of skin microcirculation because blood flow and microvascular reactivity at this level are likely to be controlled by humoral (non-neural) factors (e.g. vasoactive metabolites and tissue oxygen) s.9,547. $^{319}$. Hence, if skin blood flow would be disturbed more at the thermoregulatory than at the nutritive level of skin microcirculation, neural mechanisms are more likely to be involved than humoral mechanisms.

\section{PATIENTS AND METHODS}

\section{Patients}

Fifty-four patients with unilateral complaints met the standards for RSD described in chapter 4. of the upper extremity and 16 controls. The age of the 54 RSD patients (21 males, 33 females) ranged from 21 to 74 years, with a mean value of 48 years. Patients with a stationary warnth sensation were categorised stage 1 , patients with an intermittent warmth and cold sensation were categorised stage II, and in case of a stationary cold sensation, patients were categorised stage III. Events precipitating RSD were fractures $(50 \%)$, soft tissue traumas $(18 \%)$, operative procedures $(8 \%)$, neurovascullar compressions $(15 \%)$, cerebrovascular accidents ( $2 \%$ ), and whiplash traumas ( $2 \%$ ). In $7 \%$ of the patients, no precipitating event could be traced. Smoking habits, age, and duration of disease are shown, per stage, in table 8.1. A group of 16 age-matched volunteers was investigated as controls.

\begin{tabular}{lllll}
\hline STAGE & N & $\begin{array}{l}\text { SM } \\
(\%)\end{array}$ & $\begin{array}{l}\text { MEAN AGE } \\
\text { (years, range) }\end{array}$ & $\begin{array}{l}\text { MEAN DURATION OF DISEASE } \\
\text { (months, range) }\end{array}$ \\
\hline Con & 16 & 31 & $47(21-69)$ & \\
I & 12 & 58 & $47(24-74)$ & $3(1-7)$ \\
II & 32 & 47 & $49(21-73)$ & $9(2-28)$ \\
III & 10 & 70 & $46(31-65)$ & $47(2-241)$ \\
\hline
\end{tabular}

Table 8.1: patient characteristics: per stage absolute number $(N)$, percentage smokers $(S M)$, age (mean and range), and duration of syndrome (mean and range). 


\section{Experimental protocol}

The extremities of the patients were placed at heart level. The laser Doppler probe holder was attached to the pulp of a finger on the clinically unaffected side matching the most symptomatic finger on the clinically affected side. LDF measurements were performed at unheated skin. Simultaneously, skin temperature was measured at the dorsum of the same digit. LDF was assessed under resting conditions and during reactive hyperaemia, following release of three minutes arterial occlusion. The measurements at heart level were followed by the same measurements with the hand in the dependent position ( 35 centimetres below heart level). Subsequently, the patient was seated in a chair, with the hand placed on the stage of the microscope, again at heart level. On the clinically unaffected side, capillary microscopy was performed in the nailfold of the finger matching the most symptomatic finger of the clinically affected hand. Images of capillaries were recorded. Subsequently, complete arterial occlusion was induced with an inflatable cuff around the upperarm. After acute deflation of the cuff images were recorded during reactive hyperaemia. Reactive hyperaemia was used because it improves the reproducibility of the assessment of microcirculatory parameters ${ }^{518}$. Then the arm was lowered until the hand was 35 centimetres below heart level. After stabilisation of blood flow, the foregoing measurements were repeated. The same protocol was followed for the control subjects, using the fourth finger of the left hand.

\section{RESULTS}

Laser Doppler flowmetry (table 8.2)

\section{At heart level}

In the clinically unaffected hand, at stage I, total skin blood flow, both under resting conditions (LDFr) and at peak of reactive hyperaemia (LDFp, see table IV), was increased as compared to controls (LDFr $\mathrm{P}<0.05 ; \mathrm{LDFp} \mathrm{P}<0.05$ ). At stages II and III, no differences were observed in these parameters between the clinically unaffected hand and controls. In the clinically unaffected hand, at stage I, the ratio of rest flow at heart level and rest flow in the dependent position (R-LDFr) was lower than in controls $(\mathrm{P}<0.05)$. At stages II and III no differences could be observed in R-LDFr in the clinically unaffected hand as compared to controls.

\section{Capillary microscopy (table 8.3 )}

In the clinically unaffected hand, both red blood cell velocity at rest $(\mathrm{RBCV})$ and red blood cell velocity at peak of reactive hyperaemia $(\mathrm{RBCVp})$ did not differ from controls, at any stage of RSD. In the clinically unaffected hand, the ratio of rest velocity at heart levell and rest velocity in dependent position (R-RBCVr) did not differ from controls, at any stage of RSD.

\section{Skin temperature (table 8.2 )}

In the clinically unaffected hand, at stage I, skin temperature was increased as compared to controls $(\mathrm{P}<0.05)$. At stages II and III, no differences could be observed in this parameter between the clinically unaffected hand and controls. 


\begin{tabular}{|c|c|c|c|c|}
\hline & CONT. & STAGE I & STAGE \| & STAGE III \\
\hline $\begin{array}{l}\text { rest flow } \\
\text { (heart level) }\end{array}$ & $\begin{array}{l}81 \\
(51-109)\end{array}$ & $\begin{array}{l}137^{*} \\
(77-190)\end{array}$ & $\begin{array}{l}76 \\
(58-117)\end{array}$ & $\begin{array}{l}92^{4} \\
(49-112)\end{array}$ \\
\hline $\begin{array}{l}\text { peak flow } \\
\text { (heart level) }\end{array}$ & $\begin{array}{l}125 \\
(79-147)\end{array}$ & $\begin{array}{l}180^{\circ} \\
(99-285)\end{array}$ & $\begin{array}{l}130 \\
(83-166)\end{array}$ & $\begin{array}{l}136 \\
(82-174)\end{array}$ \\
\hline $\begin{array}{l}\text { rest flow } \\
\text { (dependent) }\end{array}$ & $\begin{array}{l}37 \\
(9-48)\end{array}$ & $\begin{array}{l}106^{109 x} \\
(59-120)\end{array}$ & $\begin{array}{l}43 \\
(26-74)\end{array}$ & $\begin{array}{l}48 \\
(20-82)\end{array}$ \\
\hline $\begin{array}{l}\text { peak flow } \\
\text { (dependent) }\end{array}$ & $\begin{array}{l}66 \\
(51-117)\end{array}$ & $\begin{array}{l}124^{\circ} \\
(106-184)\end{array}$ & $\begin{array}{l}98 \\
(73-156)\end{array}$ & $\begin{array}{l}117 \\
(48-157)\end{array}$ \\
\hline $\begin{array}{l}\text { vasoconstrictor } \\
\text { response }\end{array}$ & $\begin{array}{l}1.8 \\
(1.4-8.1)\end{array}$ & $\begin{array}{l}1.3^{*} \\
(1.1-2.3)\end{array}$ & $\begin{array}{l}1.6^{* 4} \\
(1.2-3.0)\end{array}$ & $\begin{array}{l}1.6^{*} \\
(1.1-2.5)\end{array}$ \\
\hline skin temperature & $\begin{array}{l}31.6 \\
(31.0-33.1)\end{array}$ & $\begin{array}{l}33.2^{\circ} \\
(322-34.1)\end{array}$ & $\begin{array}{l}32.8 \\
(31.2-33.5)\end{array}$ & $\begin{array}{l}31.2^{m} \\
(29.6-31.9)\end{array}$ \\
\hline
\end{tabular}

Table 8.2: data obtained by laser Doppler fowmetry and skin temperature measurement at heart level and in the dependent position, for both individual patient groups (unaffected hand) and controls. Differences between clinically unaffected side and controls: " $P<0.05$, $" P<0.001$. CONT, $=$ controls.

\begin{tabular}{|c|c|c|c|c|}
\hline & CONT. & STAGE I & STAGE II & STAGE III \\
\hline $\begin{array}{l}\text { rest velocity } \\
\text { (heart level) }\end{array}$ & $\begin{array}{l}318 \\
(230-491)\end{array}$ & $\begin{array}{l}341 \\
(237-703)\end{array}$ & $\begin{array}{l}258^{\#} \\
(134-466)\end{array}$ & $\begin{array}{l}289^{10} \\
(233-373)\end{array}$ \\
\hline $\begin{array}{l}\text { peak velocity } \\
\text { (heart level) }\end{array}$ & $\begin{array}{l}762 \\
(518-1157)\end{array}$ & $\begin{array}{l}791 \\
(590-1561)\end{array}$ & $\begin{array}{l}822 \\
(622-1151)\end{array}$ & $\begin{array}{l}622^{10} \\
(552-827)\end{array}$ \\
\hline $\begin{array}{l}\text { rest velocity } \\
\text { (dependent) }\end{array}$ & $\begin{array}{l}163 \\
(108-270)\end{array}$ & $\begin{array}{l}198 \\
(168-3.14)\end{array}$ & $\begin{array}{l}112 \\
(71-183)\end{array}$ & $\begin{array}{l}129 \\
(58-323)\end{array}$ \\
\hline $\begin{array}{l}\text { peak velocity } \\
\text { (dependent) }\end{array}$ & $\begin{array}{l}799 \\
(529-903)\end{array}$ & $\begin{array}{l}903 \\
(603-1060)\end{array}$ & $\begin{array}{l}782 \\
(368-920)\end{array}$ & $\begin{array}{l}626 \\
(473-744)\end{array}$ \\
\hline $\begin{array}{l}\text { vasoconstrictor } \\
\text { response (rest) }\end{array}$ & $\begin{array}{l}2.1 \\
(1.2-2.8)\end{array}$ & $\begin{array}{l}1.8 \\
(1.4-2.9)\end{array}$ & $\begin{array}{l}2.3 \\
(1.0-3.4)\end{array}$ & $\begin{array}{l}1.8^{\#} \\
(1.5-3.8)\end{array}$ \\
\hline
\end{tabular}

Table 8.3: data obtained by capillary microscopy, at heart level and in the dependent position, for both individual patient groups (unaffected hand) and controls. $C O N=$ controls. 


\section{DISCUSSION}

The present study shows that in stage I RSD patients thermoregulatory skin blood flow is increased and that the vasoconstrictor response to dependency is impaired in the clinically unaffected hand. This indicates the involvement of a spinal component in the microcirculatory abnormalities at this stage of the RSD syndrome. It has been demonstrated previously that excitation of a peripheral nerve can increase blood flow in the contralateral extremity. Blumberg ${ }^{53}$ and Loven ${ }^{338}$ observed, in himans and rabbits respectively, that nerve stimulation provolkes vasodilatation not only in the ipsilateral, but also in the contralateral extremity through a neural reflex mechanism. Hence, the increase in thermoregulatory skin blood flow in the clinically unaffected as well as the clinically affected hand of stage I RSD patients may have resulted from such a neural reflex mechanism, initiated by (post-) traumatic excitation of a peripheral nerve on the clinically affected side. The presence of microcirculatory abnormalities render the clinically unaffected hand unfit to serve as control for studies on thermoregulatory skin blood flow in the clinically affected hand of stage I RSD patients. Moreover, these findings are in keeping with the hypothesis that neuropathophysiological mechanisms play a role in RSD.

Several neural connections between the spinal cord and the clinically unaffected side may be responsible for the microcirculatory abnormalities as observed on this side. Kerouac and colleagues ${ }^{272}$ demonstrated that administration of substance $P$ at spinal level generates, trans-synaptically, antidromic nerve impulses in nociceptive nerve fibres. These antidromic nerve impulses have been reported, both in humans ${ }^{392}$ and in rats ${ }^{174}$, to induce vasodilatation of skin microvessels through release of vasoactive compounds such as substance $\mathrm{P}$ and CGRP at peripheral terminals of capsaicin sensitive $\mathrm{C}$-fibre afferents ${ }^{174}$. Hence, the observed increase in thermoregulatory skin blood flow on the clinically unaffected side of stage I RSD patients may result from antidromic vasodilator impulses in nociceptive nerve fibres on this side. These antidromic vasodilator nerve impulses may be generated in the spinal cord consequent to (post-) traumatic excitation of a peripheral nerve on the clinically affected side. On the basis of the findings in this study we cannot exclude, however, that a decrease in efferent sympathetic vasoconstrictor nerve impulses from the spinal cord to the clinically unaffected side has contributed to the observed increase in thermoregulatory skin blood flow at stage I, since it has been demonstrated previously, in humans, that sympathectomy increases skin blood flow ${ }^{314,510}$. An additional argument for a decrease in efferent sympathetic vasoconstrictor nerve impulses on the clinically unaffected side of stage I RSD patients is given by the fact that the vasoconstrictor response to dependency was impaired in stage I RSD patients, since this response is considered to be predominantly sympathetically mediated ${ }^{519}$. The observation, however, that activation of nociceptive afferents on one side induces an increase in the activity of postganglionic sympathetic efferents on the contralateral side ${ }^{438,559}$ argues against the hypothesis of a decrease in efferent sympathetic vasoconstrictor nerve impulses from the spinal cord to the clinically unaffected side.

The finding that skin blood flow on the clinically unaffected side was not disturbed at the humorally controlled ${ }^{59,547}$ nutritive level of skin microcirculation, in contrast to the observed disturbance at the mainly neurally controlled ${ }^{219,547}$ thermoregulatory level; suggests 
that the purported antidromic mechanisms act through trans-synaptic neural regulation rather than through hormones released into the circulation at the spinal level.

The disturbance of the vasoconstrictor response to dependency as observed in our study in the clinically unaffected hand of stage I RSD patients may have been caused by an impaired reflex control of vasoconstrictor neurons at the spinal level. The latter has been demonstrated in a cat model to result from nerve injury ${ }^{52}$.

Our microcirculatory findings at later stages of the RSD syndrome are in line with those of previous studies that could not demonstrate differences, neither in resting skin blood flow $^{35,442}$ nor in the vasoconstrictor response to dependency ${ }^{4,42}$, between the clinically unaffected extremities of RSD patients and extremities of healthy controls at later stages of the RSD syndrome. They missed the early changes, because they did not assess, in a sufficient number of patients, abnormalities in these responses at an early stage of the RSD syndrome.

At stages II and III, no abnormalities in skin blood flow or microvascular reactivity to dependency could be detected in the clinically unaffected hand. So far, we do not have a satisfactory explanation for this observation. In contrast, in the clinically affected hand, microcirculatory abnormalities are present at all stages of the syndrome ${ }^{299}$. This predominance of microcirculatory abnormalities in the clinically affected hand, however, suggests that the spinal component to the microcirculatory abnormalities in the clinically unaffected hand is secondary to an abnormal afferent imput from the clinically affected hand. "The neuropathophysiological mechanisms underlying RSD, therefore, most likely are primarily of peripheral origin. Hence, it is tempting to speculate that the mechanisms underlying RSD are quite similar to the neuropathophysiological mechanisms in the chronic constriction injury (CCI) model in the rat, as described by Bennett and Xie ${ }^{40}$. In this model, signs and symptoms of RSD can be induced by loose ligation of a sciatic nerve, which induces axonal degeneration of myelinated as well as unmyelinated nerve fibres ${ }^{31.69}$. The hypothesis that peripheral (compressive) neuropathy plays an important role in RSD is supported by numerous studies reporting on the presence of compressive nerve lesions in RSD patients $202,304,371,401$.

As alluded to before, the alleged antidromic vasodilator mechanisms on the clinically unaffected side may act through release of neuropeptides such as substance $P$ and CGRP. These neuropeptides have additionally been reported to increase the excitability of primary sensory nerve fibres ${ }^{132}$, which may explain the previously reported presence of sensory disturbances in the clinically unaffected extremity of some RSD patients ("mirror imaging") ${ }^{294_{4} 463}$. This hypothesis is in keeping with the findings of Levine and colleagues ${ }^{321}$, who demonstrated in a rat model that an abnormal afferent input, resulting from injury to one hind paw, can provoke hyperalgesia in the contralateral hind paw via neural connections across the spinal cord, a process called reflex neurogenic inflammation. Additionally, the spinal component to the observed skin blood flow abnormalities may be associated with the reported predisposition of RSD patients to develop the syndrome in the previously non-symptomatic extremity ${ }^{443}$.

The present study shows that microcirculatory abnomalities exist at the thermoregulatory level of the skin microcirculation in the clinically unaffected hand of stage I RSD patients. This indicates that there is a spinal component to microcirculatory abnomalities in RSD. This spinal component most likely acts through antidromic vasodilator nerve impulses in nociceptive nerve fibres on the clinically unaffected side and may be initiated by (post-) traumatic excitation of a peripheral nerve on the clinically affected side. 


\section{Chapter 9}

REFLEX SYMPATHETIC DYSTROPHY:

DOES SYMPATHETIC DYSFUNCTION ORIGINATE FROM PERIPHERAL NEUROPATHY?

Kurvers HAJM, Hofstra I, Jacobs MJHM, Daemen MARC, Wildenberg van den FAJM, Kitslaar PJEHM, Slaaf DW, Reneman RS

Departments of Surgery of the University Hospital Maastricht and of the Academic Medical Centre Amsterdam, The Netherlands.

Departments of Surgery, Biophysics and Physiology of the Cardiovascular Research Institute Maastricht (CARIM), The Netherlands.

Based on: Surgery, 1996, vol. 119, p. 288-296. 


\section{SUMMARY}

Sympathetic dysfunction in reflex sympathetic dystrophy (RSD) has been purported to consist of an afferently-induced increase in sympathetic nerve impulses (somato-sympathetic reflex) and/or denervation-induced hypersensitivity of the vasculature and/or sensory afferents to catecholamines. In addition, both the central and peripheral nervous system have been claimed to be involved. It was the aim of the present study to obtain more insights into these underlying mechanisms. In the affected extremities of 42 RSD patients we investigated as indirect measures of sympathetic (dys-) function: 1) skin blood flow and the vasoconstrictive response to dependency of skin microvessels by means of laser Doppler flowmetry (distal to the site of trauma), 2) relative distension of the brachial artery and changes in relative distension consequent to a cold pressor test (CPT) by means of ultrasonic vessel wall tracking (proximal to the site of trauma), and 3) arterial blood pressures by means of Finapres. Both provocation tests induce a sympathetically mediated response. Patients were divided into three categories according to their perception of skin temperature in their injured limb (stage I: stationary warnth sensation; stage II: intermittent warm th and cold sensation; stage HI: stationary cold sensation). Distal to the site of trauma, when compared with controls, skin blood flow was increased at stage I and decreased at stages II and III, whereas the vasoconstrictive response to dependency was impaired at all three stages. Proximally, when compared with controls, relative distension of the brachiall artery as well as its response to the CPT were decreased at all three stages. No differences were observed in pulse pressure between patient groups and controls. These results suggest that sympathetic dysfunction in extremities of RSD patients distal to the site of trauma consists of hypersensitivity to catecholamines at stages II/III consequent to autonomic denervation at stage I, whereas proximal to the site of trauma sympathetic nerve impulses may be increased at all three stages.

\section{INTRODUCTION}

Reflex sympathetic dystrophy (RSD) is an incompletely understood pain syndrome, which is usually localised in the extremities. It is characterised by a triad of autonomic (sympathetic), sensory, and motor disturbances $^{50}$. It has been suggested frequently that an increase in sympathetic nerve impulses plays a pivotal role in the pathophysiology of the disorder ${ }^{57,134,417}$. Such an increase may result from activation of nociceptive afferents at the site of injury, which has been reported to produce marked increases in sympathetic nerve impulses ${ }^{34,48,288}$. However, treatment of RSD aimed at interrupting this alleged increase in sympathetio nerve impulses by means of sympathectomy is not invariably satisfactory ${ }^{770}$. This might be explained by some recent studies indicating that the affected extremities of RSD patients are hypersensitive to catecholamines 19 , consequent to autonomic (sympathetic) denervation ${ }^{138,298,299}$. Additionally, controversy exists as to the origin of sympathetic dysfunction in RSD; both the peripheral and the central nerwous system have been purported to be involwed ${ }^{417}$.

Under resting conditions, blood flow at the thermoregulatory level of skim microcirculation is strongly sympathetically controlled ${ }^{19}$. Besides, at this level of skin microcirculation it has been demonstrated that the vasoconstrictive response, which is induced by changing the position of an extremity from heart level to dependency, is sympathetically mediated ${ }^{19}$. As a result, sympathetic denervation causes impairment of this response $\mathrm{e}^{219}$. To investigate sympathetic dysfunction, if any, distal to the site of the precipitating trauma, we examined skin blood flow as well as the vasoconstrictive response to dependency of skin microvessels by means of laser Doppler flowmetry (LDF). LDF was used to study the microcirculation because arteriovenous anastomoses and the subpapillary plexus, 
which are richly imnervated by sympathetic nerve endings ${ }^{319}$, contribute predominantly to the flow signal recorded with this technique ${ }^{193}$.

To obtain insight into the sympathetic disorder proximal to the site of the precipitating trauma; if any, skin blood flow, as measured by means of LDF, could also have been used as indicator. However, it has been reported previously ${ }^{478}$ that the richly sympathetically innervated arteriovenous anastomoses, which predominantly contribute to the flow signal recorded with the LDF technique ${ }^{153}$, are found solely in the skin of the palmar side of the hands ${ }^{478}$. Hence, a LDF signal obtained proximal to the wrist (site of precipitating trauma) may not be an adequate measure of sympathetio (dys-) function. In a previous study we demonstrated an extensive influence of the sympathetic nervous system on distensibility of the brachial artery ${ }^{297}$. A decrease in distension of this brachial artery was observed: following contralateral application of a cold pressor test, which increases sympathetic nerve impulses ${ }^{319}$. Additionally, it has been demonstrated by Kawisaka and colleagues ${ }^{26}$ that cooling induces a decrease in distension of the brachial artery. To investigate sympathetic dysfunction proximal to the site of the precipitating trauma we, therefore, investigated distension of the brachial artery in the clinically affected arm and changes in this parameter consequent to a cold pressor test (CPT) by means of ultrasonic vessel wall tracking (UVWT). The CPT evolkes sympathetic stimulation ${ }^{438}$. It has been demonstrated in humans that the consequent hemodynamic responses are mediated by stimulation of sympathetic nerves rather than stimulation of adrenal glands ${ }^{319}$. Hence, an increase in stiffness of the brachial artery following the CPT may be used as a measure of autonomic (sympathetic) innervation.

If autonomic dysfunction in RSD results from damage to sympathetic axons (distal sympathetic denervation) at the site of the precipitating trauma, a discongruity in sympathetic dysfunction may be expected between sites proximal to and distal to this trauma.

\section{PATIENTS AND METHODS (for detailed information the reader is referred to chapter 4)}

\section{Patients}

Forty-two RSD patients, with unilateral complaints, met the standards described in chapter 4. The age of the 15 males and 27 females ranged from 21 to 72 years, with a mean age of 48 years. The traumatic events precipitating RSD were a wrist fracture in 27 patients, a soft tissue trauma near the wrist in another 12 patients, and a carpal tunnel syndrome in the remaining 3 patients. Snoking habits, age, and duration of the RSD syndrome per stage are displlayed in table 2. A group of 16 age and gender matched healthy volunteers served as controls.

\section{Experimental protocol}

The affected arm of the patient was placed at heart level. LDF measurements were performed at unheated skin under resting conditions to assess skin blood flow under physiological conditions. Then the arm was lowered until the fingers were 35 centimetres below heart level in order to assess microvascular reactivity. After stabilisation of blood flow LDF was assessed during three minutes. Vessel wall characteristics were assessed in the brachial artery, with the subjects in the supine position. Simultaneously, arterial blood pressures were assessed by means of Finapres. To this end, the cuff was applied to the middle phalanx of the second finger. Measurements were performed before and after emerging of the clinically non-affected hand, to the level of the wrist, into water at $0^{\circ} \mathrm{C}$ for 1 minute (cold pressor test, CPT) in order to test reactivity of the brachial artery. 


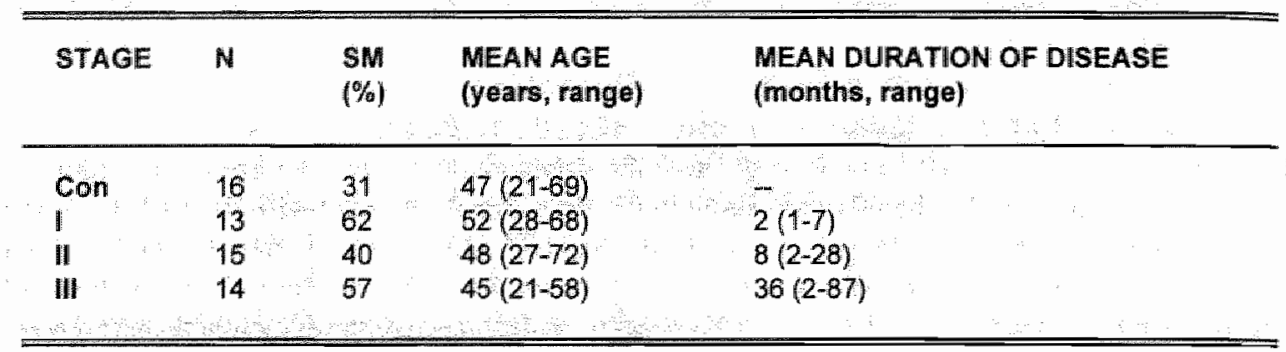

Table 9.1: patient characteristics: per stage absolute number ( $N$ ), percentage smokers (SM) age (mean and range), and duration of syndrome (mean and range).

\section{RESULTS}

\section{Laser Doppler flowmetry (table 9.2)}

As compared to controls, basal skin blood flow evolved from an increase at stage I of the RSD syndrome $(\mathrm{P}<0.001)$ to a decrease at stages II $(\mathrm{P}<0.05)$ and III $(\mathrm{P}<0.001)$. When the position of the hand was changed from heart level to the dependent position skin blood flow decreased in the control subjects ( $\mathrm{P}<0.001$ ), resulting in a heart level to dependent flow ratio of 1.82 . As compared to controls, the reduction of flow was far less at all stages of the RSD syndrome $(P<0.01)$. In the dependent position, as compared to controls, skin blood flow was increased at stage I of the RSD syndrome ( $P<$ 0.001 ) and did not differ at stages II and III.

\begin{tabular}{|c|c|c|c|c|c|c|c|c|}
\hline & \multicolumn{2}{|c|}{ controls } & \multicolumn{2}{|c|}{ stage I } & \multicolumn{2}{|c|}{ stage II } & \multicolumn{2}{|c|}{ stage III } \\
\hline & HL & dep & HL & dep & $\mathrm{HL}$ & dep & HL. & dep \\
\hline SBF & $\begin{array}{l}81 \\
(51- \\
109)\end{array}$ & $\begin{array}{l}39 \\
(9- \\
49)\end{array}$ & $\begin{array}{l}125^{\text {tise }} \\
(100- \\
202)\end{array}$ & $\begin{array}{l}91 \\
(77- \\
139)\end{array}$ & $\begin{array}{l}40^{*} \\
(36- \\
72)\end{array}$ & $\begin{array}{l}37 \\
(13- \\
53)\end{array}$ & $\begin{array}{l}31 \cdots \\
(16- \\
47)\end{array}$ & $\begin{array}{l}27 \\
(14- \\
37)\end{array}$ \\
\hline FR & \multicolumn{2}{|c|}{$\begin{array}{c}1.82 \\
(1.41-7.69)\end{array}$} & \multicolumn{2}{|c|}{$\begin{array}{c}1.28^{*} \\
(1.22-1.62)\end{array}$} & \multicolumn{2}{|c|}{$\begin{array}{c}1.23^{2+} \\
(1-1.57)\end{array}$} & \multicolumn{2}{|c|}{$\begin{array}{c}1.2^{\prime \prime} \\
(0.9-1.9)\end{array}$} \\
\hline
\end{tabular}

Table 9.2: laser Doppler flowmetry data for patient groups and controls Asterisks represent differences when compared with controls; ${ }^{P}<0.05$, ${ }^{*} P<0.01, "-P<0.001$. SBF $=$ skin blood flown $H L=$ heart level; Dep $=$ dependent; $F R=$ heart level to dependent flow ratio. 


\section{Vessel wall tracking (table 9.3)}

Before application of the cold pressor test, as compared to controls, the diameter of the brachial artery did not differ at stages I and $\mathbb{I}$, but was decreased at stage III $(\mathrm{P}<0.01)$. The distension of the brachial artery, as compared to controls, tended to be decreased at stage I $(\mathrm{P}=0.06)$ and stage $\mathrm{II}(\mathrm{P}=0.09)$, and was significantly decreased at stage III ( $\mathrm{P}<0.001$ ). Relative distension of the brachiall artery, as compared to controls, was decreased at all stages of the RSD syndrome (stages I and II, P $<0.05$; stage III, $\mathrm{P}<0.01$ ). When the cold pressor test was applied to the clinically unaffected hand, relative distension of the brachial artery decreased $(P<0.001)$ in the control subjects, resulting in a relative distension ratio of 1.43 . As compared to controls, the reduction in relative distension was less at all stages of the RSD syndrome (stage $\mathbb{I}, \mathrm{P}<0.01$; stage $\mathbb{I}, \mathrm{P}<0.01$; stage $\mathbb{I I I}, \mathrm{P}<0.05$ ). Following application of the cold pressor test, as compared to controls, the diameter of the brachial artery did not differ at stages I and II, and tended to be decreased at stage III $(\mathrm{P}=0.06)$. The distension of the brachial artery, as compared to controls, did not differ at stages I and II, but was decreased at stage III $(\mathrm{P}<0.05)$. Relative distension of the brachial artery, again as compared to controls, did not differ at stages I and II of the RSD syndrome and tended to be decreased at stage III $(\mathrm{P}=0.05)$.

\begin{tabular}{|c|c|c|c|c|c|c|c|c|}
\hline & \multicolumn{2}{|c|}{ controls } & \multicolumn{2}{|l|}{ stage I } & \multicolumn{2}{|l|}{ stage II } & \multicolumn{2}{|c|}{ stage Ill } \\
\hline & $\begin{array}{l}\text { before } \\
\text { CPT }\end{array}$ & $\begin{array}{l}\text { after } \\
\text { CPT }\end{array}$ & $\begin{array}{l}\text { before } \\
\text { CPT }\end{array}$ & $\begin{array}{l}\text { after } \\
\text { CPT }\end{array}$ & $\begin{array}{l}\text { before } \\
\text { CPT }\end{array}$ & $\begin{array}{l}\text { after } \\
\text { CPT }\end{array}$ & $\begin{array}{l}\text { before } \\
\text { CPT }\end{array}$ & $\begin{array}{l}\text { after } \\
\text { CPT }\end{array}$ \\
\hline diameter & $\begin{array}{l}3630 \\
(3450- \\
3955)\end{array}$ & $\begin{array}{l}3360 \\
(3110 \\
4315)\end{array}$ & $\begin{array}{l}3770 \\
(2850- \\
4020)\end{array}$ & $\begin{array}{l}3650 \\
(2905 \\
4030)\end{array}$ & $\begin{array}{l}3790 \\
(3367 \\
4034)\end{array}$ & $\begin{array}{l}3630 \\
(2895 \\
4357)\end{array}$ & $\begin{array}{l}2935 \\
(2450 \\
3290)\end{array}$ & $\begin{array}{l}3070 \\
(2392- \\
3425)\end{array}$ \\
\hline $\begin{array}{l}\text { absolute } \\
\text { distension }\end{array}$ & $\begin{array}{l}126 \\
(96 \\
151)\end{array}$ & $\begin{array}{l}95 \\
(48- \\
116)\end{array}$ & $\begin{array}{l}102 \\
(77 \\
122)\end{array}$ & $\begin{array}{l}74 \\
(71 \\
123)\end{array}$ & $\begin{array}{l}101 \\
(78 \\
137)\end{array}$ & $\begin{array}{l}100 \\
(75 \\
136)\end{array}$ & $\begin{array}{l}63^{4} \\
(38 \\
86)\end{array}$ & $\begin{array}{l}49^{\prime} \\
(39 \\
56)\end{array}$ \\
\hline $\begin{array}{l}\text { relative } \\
\text { distension }\end{array}$ & $\begin{array}{l}3.33 \\
(2.95 \\
4.31)\end{array}$ & $\begin{array}{l}2.22 \\
(1.97- \\
3.6)\end{array}$ & $\begin{array}{l}2.9^{\circ} \\
(2.1 \\
3.37)\end{array}$ & $\begin{array}{l}2.45 \\
(1.76 \\
3.02)\end{array}$ & $\begin{array}{l}2.49 \\
(1.89 \\
3.92)\end{array}$ & $\begin{array}{l}2.51 \\
(2.33- \\
3.57)\end{array}$ & $\begin{array}{l}2.07^{*} \\
(1.27- \\
2.97)\end{array}$ & $\begin{array}{l}1.67 \\
(1.25 \\
2.75)\end{array}$ \\
\hline $\begin{array}{l}\text { ratio } \\
\text { relative } \\
\text { distenston }\end{array}$ & \multicolumn{2}{|c|}{$\begin{array}{c}1.43 \\
(1.21-1.69)\end{array}$} & \multicolumn{2}{|c|}{$\begin{array}{c}4.1^{*} \\
(0.92-1.44)\end{array}$} & \multicolumn{2}{|c|}{$\begin{array}{c}0.94^{\circ+} \\
(0.66-1.45)\end{array}$} & \multicolumn{2}{|c|}{$\begin{array}{c}1.08^{*} \\
(0.9 \cdot 1.78)\end{array}$} \\
\hline
\end{tabular}

Table 9.3: ultrasonic vessel wall tracking data for patient groups and controls. Asterisks represent differences when compared with controls; $" P<0.05, " P<0.01, "-P<0.001$. Dist. $=$ distension. $C P T=$ cold pressor test.

\section{Finapres (table 9.4)}

Before the cold pressor test, no differences were observed in systolic arterial blood pressure or pulse pressure between controls and patient groups. As compared to controls, diastolic arterial blood pressure tended to be increased $(\mathrm{P}=0.052)$ at stage III, but did not differ at stages $/ /$ II. Following the cold pressor test, no differences were observed in systolic arterial blood pressure, diastolic arterial blood pressure, or pulse pressure between patient groups and controls. Pulse pressure did not differ between patient groups and controls, neither before nor after the cold pressor test. Therefore, relative distension of the brachial artery may be considered as an appropriate measure of changes in local wessel wall properties. 


\begin{tabular}{|c|c|c|c|c|c|c|c|c|}
\hline & \multicolumn{2}{|l|}{ controls } & \multicolumn{2}{|l|}{ stage I } & \multicolumn{2}{|l|}{ stage II } & \multicolumn{2}{|c|}{ stage Ill } \\
\hline & $\begin{array}{l}\text { before } \\
\text { CPT }\end{array}$ & $\begin{array}{l}\text { after } \\
\text { CPT }\end{array}$ & $\begin{array}{l}\text { before } \\
\text { CPT }\end{array}$ & $\begin{array}{l}\text { after } \\
\text { CPT }\end{array}$ & $\begin{array}{l}\text { before } \\
\text { CPT }\end{array}$ & $\begin{array}{l}\text { after } \\
\text { CPT }\end{array}$ & $\begin{array}{l}\text { before } \\
\text { CPI }\end{array}$ & $\begin{array}{l}\text { after } \\
\text { CPT }\end{array}$ \\
\hline $\mathrm{SBP}$ & $\begin{array}{l}133 \\
(122 \\
137)\end{array}$ & $\begin{array}{l}156 \\
(142 \\
165)\end{array}$ & $\begin{array}{l}135 \\
120 \\
150)\end{array}$ & $\begin{array}{l}155 \\
(135- \\
161)\end{array}$ & $\begin{array}{l}130 \\
(116- \\
158)\end{array}$ & $\begin{array}{l}157 \\
(138 \\
175)\end{array}$ & $\begin{array}{l}133 \\
(116- \\
162)\end{array}$ & $\begin{array}{l}145 \\
(142 \\
168)\end{array}$ \\
\hline DBP & $\begin{array}{l}70 \\
(68-79)\end{array}$ & $\begin{array}{l}83 \\
(79-94)\end{array}$ & $\begin{array}{l}72 \\
(67-82)\end{array}$ & $\begin{array}{l}89 \\
(71-96)\end{array}$ & $\begin{array}{l}71 \\
(66-98)\end{array}$ & $\begin{array}{l}86 \\
(83-99)\end{array}$ & $\begin{array}{l}82 \\
(67-87)\end{array}$ & $\begin{array}{l}85 \\
(77-91)\end{array}$ \\
\hline$P P$ & $\begin{array}{l}56 \\
(53-62)\end{array}$ & $\begin{array}{l}66 \\
(60-73)\end{array}$ & $\begin{array}{l}61 \\
(55-70)\end{array}$ & $\begin{array}{l}64 \\
(62-70)\end{array}$ & $\begin{array}{l}5.3 \\
(44-61)\end{array}$ & $\begin{array}{l}71 \\
(56-79)\end{array}$ & $\frac{52}{(34-81)}$ & $\begin{array}{l}62 \\
(51-83)\end{array}$ \\
\hline
\end{tabular}

Table 9.4: Finapres data for patient groups and controls. $S B P=$ systolic blood pressure; $D B P=$ diastolic blood pressure; $\mathrm{PP}=$ pulse pressure; $\mathrm{CPT}=$ cold pressor test

\section{DISCUSSION}

The observation that skin blood flow in the hand is increased at stage I and reduced at stages II and III of RSD is compatible with autonomic denervation followed by increased sensitivity to catecholamines distal to the site of injury. In concert with autonomic denervation is the impaired sympathetically mediated wasoconstrictive response upon dependency at all stages of the syndrome. Our hypothesis of sympathetic denervation, distal to the site of trauma precipitating RSD, is supported by a study of Drummond and colleagues ${ }^{138}$, who observed a decrease in venous plasma levels of noradrenalline, in blood collected in the affected limb of RSD patients. The finding that sympathectomy increases basal skin blood flow ${ }^{510}$, supports the hypothesis of sympathetic denervation causing the observed increase in skin blood flow at stage I. It is well known that sympathetic denervation provokes increased sensitivity of vascular structures to catecholamines ${ }^{42,233}$. Arnold and colleagues ${ }^{19}$ presented direct invivo evidence for hypersensitivity to noradrenaline of vascular structures in limbs affected by RSD. Hence, the observed decrease in preprovocation skin blood flow at stages II and III most likely results from hypersensitivity of skin microvessels to (circulating) catecholamines. It can not be excluded that the impaired wasoconstriction upon dependency at stages Ill and III is partly influenced by the decreased skin blood flow at heart level.

Proximal to the site of trauma, the differences in vessel wall characteristics between patient groups and controls may have been caused by differences in local vessel wall properties and/or pulse pressure. Our finding that pulse pressure did not differ from controls at any stage of RSD argues against the latter. Changes in local vessel wall properties may result from an increase in sympathetic nerve impulses consequent to activation of nociceptive afferents at the site of injury (somato-sympathetic reflex ${ }^{34,288}$ or from hypersensitivity to catecholamines consequent to autonomic denervation ${ }^{33}$. At stage I of the RSD syndrome, preprovocation relative distension was decreased suggesting that proximal to the site of trauma sympathetic denervation and, hence, hypersensitivity to catecholamines does not play a role. Therefore, the decrease in preprovocation relative distension as observed at all stages of the RSD syndrome more likely results from an ongoing increase in sympathetic nerve impulses. The decrease in preprovocation relative distension, as observed at all stages of the RSD syndrome, may also account for the impaired response of the brachial artery to the cold pressor test at all stages. 
The observed incongnuty in sympathetic dysfunction indicates that the precipitating trauma prowokes not only axonal degeneration of sympathetic nerve fibres ${ }^{42}$, and thus autonomic denervation of tissues distal to the site of trauma, but also an ongoing increase in sympathetic nerve impulses proximal to the site of trauma consequent to activation of ipsilateral nociceptive afferents at the site of injury (somatosympathetic reflex) $)^{3,288}$.

As compared to controls, preprovocation relative distension was decreased at all stages of the RSD syndrome, whereas the diameter of the brachial artery was decreased only at stage III. This finding suggests that an increase in sympathetic nerve impulses has substantially mone effect on brachial artery distensibility than on brachial artery diameter. The latter is supported by our observation that, in healthy controls, application of the cold pressor test decreased distensibility of the brachial artery by approximately 33 per cent, whereas the brachial diameter was only reduced by about 7 per cent. In contrast, Vatner and colleagues ${ }^{527}$ demonstrated that $\alpha$-adrenergic stimulation not only increases distensibility but simultaneously reduces the diameter of coronary arteries. This discrepancy may have resulted from differences in experimental conditions. In our study, sympathetic stimulation was induced by means of a cold pressor tesit, whereas Vatner and colleagues induced alpha adrenergic stimulation through administration of methoxamine. Besides, their experiments were performed on coronary arteries and ours on the brachial artery.

There were substantially more habitual smokers in all of our three patient groups than in the control group. It is unlikely that this habit affects the results of our study, because it has been shown that smoking does not have a long-term effect on distensibility of peripheral vessels as the brachial and carotid arteries ${ }^{291}$.

Denervation-induced hypersensitivity to catecholamines may develop not only in skin microvessels but also in nociceptive nerve fibres ${ }^{405}$. These nociceptors thus may become more excitable and respond directly to norepinephrine. The finding that sympathectomy alone induces spontaneous pain in a sympathectomised region ${ }^{332}$, therefore, is in line with our lrypothesis that in RSD patients the phenomenon of denervation induced hypersensitivity to catecholamines may be involved not only in microcirculatory, but also in sensory abnormalities.

Both chemical and surgical sympathectomy, be it often onlly temporarily, may relieve in part pain in RSD patients ${ }^{405}$. This finding argues against the concept that pain in RSD reflects exclusively a denervation induced hypersensitivity disorder of nociceptors. The observations in our study, however, suggest that in addition to this hypersensitivity phenomenon distal to the site of trama, sympathetic nerve impulses may be ongoingly increased proximal to the site of trauma. Such an increase may induce discharge of regenerating sprouts or damaged $\mathrm{C}$-nociceptors through local release of noradrenaline at the site of traumatic nerve damage $e^{462,464}$, and, hence, may account for the aforementioned beneficial effects of sympathectomy in part of the RSD patients.

The finding that compressive nerve lesions may be present in RSD patients ${ }^{37,262,304,404}$ supports our hypothesis that RSD may be associated with traumatic peripheral nerve damage. Moreover, signs and symptoms alike those seen in RSD can be induced in a rat model by means of loose ligation of a sciatic nerve ${ }^{40}$. This procedure has been shown electron-microscopically to provoke axonal degeneration of myelinated as well as unmyelinated (sympathetic) nerve fibres at the site of ligation". Microcirculatory abnormalities in this model, as assessed distal to the site of nerve compression, are in line with those observed in RSD patients: impaired vasoconstrictive responses to an autonomic provocation test were observed from day 1 after ligation. Additionally, skin blood flow developed from an increase at an early stage to a decrease at later stages ${ }^{302}$.

Because of the alleged increase in sympathetic nerve impulses, treatment of RSD has mainly focused on blockade of these impulses by means of sympathectomy, the results of which have not been invariably satisfactory ${ }^{370}$. The concept of increased sensitivity to catecholamines consequent to sympathetic denervation, distal to the site of trauma precipitating RSD, raises the possibility of new 
therapeutic approaches such as blockade of adrenoceptors. Intravenous phentolamine ${ }^{17}$ and oral phenoxybenzarnine ${ }^{\mathrm{I} 80}$ have been demonstrated to have beneficial effects in patients with RSD. These drugs, however, may have serious side-effects such as hypotension. Future research should therefore foeus ont selective adrenergic antagonists. Additionally, the concept that RSD results from compressive (traumatic) neuropathy may raise the possibility of decompressive interventions such as surgical decompression or intravenous administration of hyperosmolar fluids in order to reduce perineural and intraneural edema.

In summary, the findings in our present study indicate that in extremities of RSD patients an incongruity exists in sympathetic dysfunction between sites proximal to and distal to the precipitating trauma, which is indicative of peripheral neuropathy at the site of trauma. Distal to this site, sympathetic dysfunction most likely consists of hypersensitivity to catecholamines consequent to autonomic (sympathetic) denervation. In contrast, proximal to the site of the precipitating trauma, sympathetic nerve impulses are most likely increased at all stages of the RSD syndrome, presumably on the basis of a somato-sympathetic reflex. 


\section{Chapter 10}

SKIN BLOOD FLOW ABNORMALITIES

IN A RAT MODEL OF PARTIAL NERVE INJURY:

RESULT OF DECREASED SYMPATHETIC VASOCONSTRICTOR OUTFLOW?

HAJM Kurvers, GJ Tangelder, JGR De Mey, DW Slaaf, RJ Beuk, FAJM van den Wildenberg, PJEHM Kitslaar, RS Reneman, MJHM Jacobs.

Departments of Surgery of the Academic Hospital Maastricht and of the Academic Medical Centre Amsterdam, The Netherlands.

Departments of Surgery, Physiology, Pharmacology, and Biophysics, Cardiovascular Research Institute Maastricht, The Netherlands.

Based on: Journal of the Autonomic Nervous System, 1997, vol. 63, p. 19-29 


\section{SUMMARY}

Loose ligation of a sciatic nerve in rats provokes signs and symptoms alike those observed in human conditions of neuropathic pain. Some of these signs and symptoms have been associated with sympathetic dysfunction. Since the skin microcirculation in the rat is strongly influenced by sympathetic tone, abnormalities in skin blood flow may be used as an indirect measure of sympathetic dysfunction. We measured, by means of laser Doppler flowmetry, skin blood flow at the plantar surface of the rat hind paw before and after ipsilateral loose sciatic nerve ligation. We assessed basal skin blood flow as well as the vasoconstrictor response which follows cooling of the rat abdomen. The effectiveness of this response may be used as a measure of sympathetic vasoconstrictor outflow. As compared to the values obtained before ligation: 1) the vasoconstrictor response was impaired from day 1 onwards, whereas 2) basal skin blood flow was increased from day 3 until day 5 , and decreased from day 7 until day 28 . These findings suggest that in the CCI model loose ligation of a sciatic nerve reduces sympathetic vasoconstrictor outflow, which, in turn may induce supersensitivity of skin microvessels to catecholamines.

\section{INTRODUCTION}

The chronic constriction injury $(\mathrm{CCl})$ model in rats, which has been developed by Bennett and $\mathrm{Xie}^{40}$ as a chronic neuropathic pain model, exhibits many of the signs and symptoms of cau salgia $^{39,40,507}$. Postoperatively, these rats develop, amongst others, behavioural signs indicative of spontaneous pain, thermal hyperalgesia, mechanical and thermal allodynia ${ }^{23}$, as well as trophic changes of muscles and claws ${ }^{40}$ of the hind paw subjected to ligation of a sciatic nerve. Many of these signs and symptoms have been associated with sympathetic dysfunction ${ }^{40,474}$, albeit that the exact role of the sympathetic nervous system sofar remains unclear.

Skin temperature in the chronic constriction injury model has been reported to evolve from an increase shortly after ligation of a nerve to a decrease at later stages ${ }^{53}$. This may reflect abnormal skin blood flow and, hence, autonomic dysfunction. The abnormal cold status of the hind paw as observed in this model at a later stage has been purported to be associated with an increase in efferent sympathetic nerve impulse ${ }^{39}$. In these CCI studies, skin temperature was used as an indirect measure of skin microcirculatory blood flow. Although thermography consistently measures thermal changes and delineates the areas of such thermal changes, it is a rather crude index of skin blood flow ${ }^{441}$. It has been demonstrated in human studies that skin blood flow can change significantly without any measurable change in skin temperature ${ }^{512}$. Besides, thermography is too slow to assess adequately instantaneous changes in skin microcirculatory blood flow as induced by autonomic provocation tests.

Therefore, we assessed skin microcirculation in the $\mathrm{CCl}$ model directly by means of laser Doppler flowmetry. Since skin microcirculatory blood flow in rat skin is influenced by sympathetic tone $\mathrm{e}^{556}$, abnormalities in skin blood flow, if any, may be used as an indirect measure of sympathetic dysfunction. Laser Doppler flowmetry allows for continuous assessment of skin blood flow and assessment of instantaneous changes in skin blood flow on 
autonomic provocation tests. Cooling of the abdomen of anaesthetised rats has been reported to provoke cutaneous vasoconstriction in the hind limbs, as indicated by a decrease in shin blood flow ${ }^{328}$. Rats subjected to lumbar sympathectomy demonstrated an impaired vasoconstrictor response to this autonomic provocation test. Ergo, the effectiveness of this response may be used as a measure of sympathetic vasoconstrictor outflow ${ }^{32 \%}$. In addition, the time course of skin temperature, neuropathic signs and symptoms, and nociceptive changes were investigated and related to the observed microcirculatory changes.

MATERIALS AND METHODS (for detailed information see chapter 4).

\section{Materials}

Of the 28 male Lewis rats used, 20 were subjected to loose ligation of the right sciatic nerve. The remaining 8 (unoperated) rats were used to obtain information on the effects of repeated measurements and increasing age/weight on skin blood flow.

\section{Experimental protocol neuropathic signs and symptoms}

In group I (ligated rats), neuropathic signs and symptoms were assessed one day before, as well as $1,3,5,7,14,21$, and 28 days after the operative procedure. The unoperated rats (group II) were subjected to the same experimental protocol: A neuropathic syndrome was considered to be present in case of an abnormal posture of the hind paw (i.e. toes kept together and ventroflexed, hind paw everted, moderate degree of foot-drop $)^{40}$. In addition to observation of the posture of the hind paw, we measured trophic changes (i.e. thickness of the lower leg muscles and length of the claws). The posture of the hind paw was assessed after the awake rats had been placed on a (thermally neutral) table under an inverted translucent plastic cage after which they were carefully observed. After the rats had been anaesthetised for assessment of skin blood flow and skin temperature, the thickness of the muscles of the lower leg (gastrocnemius/soleus) was assessed as a measure of atrophic changes. To this end, a constant pressure caliper (Mitutoyo, Tokyo, Japan) was placed half way the lower leg, with the contacts of the caliper placed on the medial and lateral side of the lower leg. This is a standard technique to assess changes in paw thickness of the rat, which are smaller than those which occurred in the present experiments ${ }^{321}$. Besides, the length of the claws was measured. To this end, an impression of these claws was made in plastic clay. Finally, the rats were weighed.

\section{Experimental protocol skin blood flow and skin temperature measurements}

In a first group of 20 rats (group I) measurements of skin temperature and laser Doppler flowmetry were performed one day before, as well as $1,3,5,7,14,21$, and 28 days after ligation of the ipsilateral sciatic nerve. This protocol was chosen in order to assess the time course of changes in skin blood flow (SBF) induced by loose sciatic nerve ligation. At these timepoints, we initially measured skin temperature. Laser Doppler Flowmetry (LDF) was employed to assess SBF. Firstly, we measured basal SBF (LDFb, in PU). Subsequently, the abdomen of the rat was cooled by spraying ethylchloride (cold pressor test, CPT). After the CPT, SBF was again measured. The ratio of basal SBF before the CPT (LDFb) divided by the 
lowest value after the CPT (LDFIv) was calculated to evaluate the effectiveness of the skin vasoconstrictor response. Besides, we measured the time to reach the lowest SBF value following the CPT (LDFt, in s). In a second group of 8 unoperated rats (group II), LDF was: performed at identical time-points as in group $\mathrm{I}$. This was done in order to obtain information on the effects of repeated measurements and increased age/weight on SBF.

\section{Experimental protocol nociceptive behavioural tests}

Withdrawal responses to thermal stimulation and behavioural signs of spontaneous pain were assessed in all ligated rats one day before, as well as $1,3,5,7,14,21$, and 28 days after the operative procedure. The unoperated rats were subjected to the same experimental protocol Since virtually all rats, which had been subjected to loose ligation of the sciatic nerve, exhibited an abnormal posture of the hind paw, it was impossible to perform the nociceptive tests blinded. However, for each testing session, the (independent) experimenter was unaware of the results of the preceding tests and of the expectations of the investigators. Observations of withdrawal responses to thermal stimulation were performed according to a procedure described in detail previously ${ }^{40,80,212}$. Briefly, the awake rats were placed on a metal floor, under an inverted translucent plastic cage. The floor was chilled $\left(4^{\circ} \mathrm{C}\right)$ or warmed $\left(35^{\circ} \mathrm{C}\right)$ by an underlying water bath. With a thermistor, attached to the floor by adhesive tape, its surface temperature was assessed. If necessary, the temperature of the water bath was adjusted. With alternating stimulus order (chilled/warmed) on subsequent experimental days, the animals were exposed to the chilled and warmed floor for five minutes. After a period of 2 minutes, during which the rats were allowed to habituate, an independent experimenter assessed the time (over a 3 minutes period) during which the rats held their right hind paw above the floor while they were sitting or standing; hind paw withdrawals related to stepping were thus not counted. Quantification of 'spontaneous' pain-related behaviour was executed according to a procedure described in detail previously ${ }^{23}$. Briefly, each animal was placed on a thermally neutral floor under an inverted translucent plastic cage, after which habituation was allowed for a few minutes. Subsequently, over a 15 minutes period the behaviour of the animal was recorded every 300 seconds in terms of a weighted pain intensity scale with a logical progression of behaviours. The scale used in this study was the following: $0=$ paw is pressed normally on the floor; $1=$ paw rests lightly on the floor and the toes are in a ventroflexed position; $2=$ only the internal edge of the paw is pressed on the floor; $3=$ only the heel is pressed on the floor and the hind paw is in an inverted position; $4=$ the whole paw is elevated; $5=$ the animal licks the operated paw. Then, using 3 consecutive score values, the mean score was calculated for each particular rat. This score provides an index of the intensity of continuous pain.

\section{RESULTS}

\section{Neuropathic signs and symptoms (table 10.1)}

An abnormal posture of the hind paw (i.e. toes kept together and ventroflexed, hind paw everted, moderate degree of foot-drop) was present in $80 \%$ of the ligated rats (group I) at day 1 after ligation. It increased to $95 \%$ of the ligated rats from day 3 onwards. In the unoperated rats (group II) an abnormal posture of the hind paw was never observed. These findings 
indicate that in our series loose ligation of the sciatic nerve almost invariably caused a peripheral neuropathy, which is in line with other studies ${ }^{23,40,533}$. Because of this consistent presence of neuropathic changes, we assessed skin blood flow and nociceptive changes in all ligated rats. When compared with preligation values, the claw length in the ligated group (group I) was firstly increased $(\mathrm{p}<0.05)$ at day 5 after ligation. Thereafter, the claw length gradually increased until the end of the experimental protocol (day 28): However, one should realise that this phenomenon may result from absence of grooming of the paralysed hind limb. In the unoperated rats (group II) claw length did not change during the whole observation period. In the ligated group (group 1 ), when compared with preligation values; the muscle thickness was firstly reduced $(p<0.05)$ at day 3 after ligation. Thereafter, a gradual further reduction was observed until the end of the experimental protocol (day 28). During the whole observation period no changes were observed in muscle thickness in the unoperated group (group II). When compared with preligation values, the weight of the rats in the ligated group (group I) was firstly increased $(p<0.05)$ at day 21 . Some minor weight loss was observed during the first week. In the unoperated group (group II), when compared with values obtained at the start of the experimental protocol, the weight of the rats was firstly increased $(p<0.05)$ at day 14. No weight loss was observed during the first week.

\begin{tabular}{|c|c|c|c|c|c|c|c|c|}
\hline & init. val. & 1 & 3 & 5 & 7 & 14. & 21 & 28 \\
\hline \multicolumn{9}{|c|}{ abnormal posture hind paw } \\
\hline lig. & 0 & 80 & 95 & 95 & 95 & 95 & 95 & 95 \\
\hline unop. & 0 & 0 & 0 & 0 & 0 & 0 & 0 & 0 \\
\hline \multicolumn{9}{|c|}{ claw length } \\
\hline lig. & $\begin{array}{l}5.0 \\
(5.0-5.2)\end{array}$ & $\begin{array}{l}5.0 \\
(5.0-5.0)\end{array}$ & $\begin{array}{l}5.0 \\
(5.0-5.2)\end{array}$ & $\begin{array}{l}5.0 \\
(5.0-6.0)\end{array}$ & $\begin{array}{l}6.0 \\
(5.0-6.0)\end{array}$ & $\begin{array}{l}6.0 \\
(6.0-6.0)\end{array}$ & $\begin{array}{l}6.0 \\
(6.0-6.5)\end{array}$ & $\begin{array}{l}6.1 \\
(5.4-6.4)\end{array}$ \\
\hline unop. & $\begin{array}{l}5.1 \\
(4.9-5.3)\end{array}$ & $\begin{array}{l}5.1 \\
(4.9-5.4)\end{array}$ & $\begin{array}{l}5.0 \\
(4.8-5.4)\end{array}$ & $\begin{array}{l}4.9 \\
(4.7-5.3)\end{array}$ & $\begin{array}{l}5.1 \\
(4.8-5.5)\end{array}$ & $\begin{array}{l}5.1 \\
(4.8-5.6)\end{array}$ & $\begin{array}{l}5.0 \\
(4.8-5.5)\end{array}$ & $\begin{array}{l}5.1 \\
(4.8-5.3)\end{array}$ \\
\hline \multicolumn{9}{|c|}{ muscle thickness } \\
\hline lig. & $\begin{array}{l}6.6 \\
(6.0-7.2)\end{array}$ & $\begin{array}{l}6.2 \\
(5.9-7.0)\end{array}$ & $\begin{array}{l}5.2 \\
(5.2-6.5)\end{array}$ & $\begin{array}{l}5.1 \\
(5.0-6.4)\end{array}$ & $\begin{array}{l}5.0 \\
(4.9-6.0)\end{array}$ & $\begin{array}{l}4.9 \\
(4.9 \cdot 6.0)\end{array}$ & $\begin{array}{l}5.0 \\
(4.8 .5 .6)\end{array}$ & $\begin{array}{l}5.1 \\
(4.8-5.7)\end{array}$ \\
\hline unop. & $\begin{array}{l}6.5 \\
(5.7-7.3)\end{array}$ & $\begin{array}{l}6.5 \\
(5.9-7.4)\end{array}$ & $\begin{array}{l}6.5 \\
(5.8-7.2)\end{array}$ & $\begin{array}{l}6.6 \\
(5.8-7.3)\end{array}$ & $\begin{array}{l}6.5 \\
(5.9-7.2)\end{array}$ & $\begin{array}{l}6.7 \\
(5.9-7.4)\end{array}$ & $\begin{array}{l}6.7 \\
(5.8-7.6)\end{array}$ & $\begin{array}{l}6.8 \\
(5.9-7.8)\end{array}$ \\
\hline \multicolumn{9}{|c|}{ weight } \\
\hline lig. & $\begin{array}{l}280 \\
(270- \\
300)\end{array}$ & $\begin{array}{l}260 \\
(250 \\
280)\end{array}$ & $\begin{array}{l}260 \\
(253- \\
280)\end{array}$ & $\begin{array}{l}260 \\
(250 \\
260)\end{array}$ & $\begin{array}{l}270 \\
(253 \\
280)\end{array}$ & $\begin{array}{l}290 \\
(280 \\
300)\end{array}$ & $\begin{array}{l}300 \\
(293 \\
300)\end{array}$ & $\begin{array}{l}300 \\
(300 \\
310)\end{array}$ \\
\hline unop. & $\begin{array}{l}270 \\
(260- \\
290) \\
\end{array}$ & $\begin{array}{l}270 \\
(250- \\
290) \\
\end{array}$ & $\begin{array}{l}260 \\
(250- \\
280) \\
\end{array}$ & $\begin{array}{l}270 \\
(260- \\
290) \\
\end{array}$ & $\begin{array}{l}290 \\
(270- \\
300) \\
\end{array}$ & $\begin{array}{l}310 \\
(290- \\
330) \\
\end{array}$ & $\begin{array}{l}330 \\
(310 \\
340)\end{array}$ & $\begin{array}{l}340 \\
(320 \\
350) \\
\end{array}$ \\
\hline
\end{tabular}

Table 10.1: time course (days) of neuropathic signs and symptoms: abnormal posture hind paw (in \%), muscle thickness (in $\mathrm{mm}$ ), and weight (in grams); median values and interquartile ranges are presented, for both the ligated and the unoperated group. Lig. = ligatedgroup; unop. = unoperated group; init. vall. = initial values. 


\section{Skin blood flow and skin temperature (table 10.2)}

The vasoconstrictor response to the cold pressor test (LDFvr) in the ligated group (group I) was impaired $(p<0.01)$ from day 1 after ligation onwards when compared with preligation values. Thereafter, no changes with time were observed in this response. During the whole observation pertod no changes were observed in the vasoconstrictor response to the cold pressor test in the unoperated group (group II). Basal skin blood flow (LDFb) in the ligated group (group I) was increased $(p<0.01)$ from day 3 until day 5 after ligation, when compared with preligation values. This increase was subsequently followed by a decrease $(p<0.0001)$ from 7 until 28 days after ligation. During the whole observation period no changes were observed in basal skin blood flow in the unoperated group (group II). The lowest skin blood flow values obtained after the cold pressor test (LDFIV) for groups I and II are presented in table 10.2 together with the basal skin blood flow values (LDFb) for comparison. In group I, the time to reach the lowest skin blood flow value after application of the cold pressor test was not influenced by loose ligation of the sciatic nerve. During the whole observation period we also did not observe changes in the time to reach the lowest skin blood flow value after application of the cold pressor test in the unoperated group (group II). In the ligated group (group I), skin temperature was decreased $(p<0.01)$ from day 1 until day 7 after ligation, when compared with preligation values.

\begin{tabular}{|c|c|c|c|c|c|c|c|c|}
\hline & IV & 1 & 3 & 5 & 7 & 14 & 21 & 28 \\
\hline $\begin{array}{l}\operatorname{lig} \\
\text { LoFw }\end{array}$ & 2.1 & $(1.2-1.7)$ & $(1.4)$ & $(1.5)$ & $(1,0-1.7)$ & $(0.6-1.4)$ & $(0.8-1.6)$ & $(1.3-2.5)$ \\
\hline unop. & 200 & $(1.4-29)$ & $(1.9-3.0)$ & 2.2 & 2,1 & $(1,3-2,4)$ & $(1.6-2.9)$ & $\frac{2.1}{(1.8-3.0)}$ \\
\hline $\begin{array}{l}\text { lig. } \\
\angle D F b\end{array}$ & $\frac{82}{(60-121)}$ & $\begin{array}{l}88 \\
(48-131)\end{array}$ & $(14200-218)$ & $\begin{array}{l}138 \\
(95-195)\end{array}$ & $(31)$ & $\begin{array}{l}48 \\
(22-66)\end{array}$ & $\begin{array}{l}43 \\
(24-71)\end{array}$ & $\begin{array}{l}26 \\
(20-41)\end{array}$ \\
\hline unop. & 84. & $\begin{array}{l}80 \\
(58-130)\end{array}$ & 82 & $\begin{array}{l}66 \\
(40-119)\end{array}$ & 74 & $\begin{array}{l}79 \\
(67-128)\end{array}$ & $91.124)$ & $\begin{array}{l}81 \\
(51-146)\end{array}$ \\
\hline $\begin{array}{l}\lg . \\
\angle O F / N\end{array}$ & $(15-68)$ & $(24)-100)$ & 96 & $\begin{array}{l}102 \\
(68-144)\end{array}$ & $(24-54)$ & $\begin{array}{l}46 \\
(27-73)\end{array}$ & 39 & $\begin{array}{l}18 \\
(13-24)\end{array}$ \\
\hline unop. & $(17 \ldots 56)$ & $\begin{array}{l}48 \\
(27-64)\end{array}$ & $\begin{array}{l}52 \\
(28.66)\end{array}$ & $\begin{array}{l}51 \\
(26-60)\end{array}$ & $420-52)$ & $\begin{array}{l}53 \\
(27-64)\end{array}$ & $\begin{array}{l}55 \\
(31.71)\end{array}$ & $\begin{array}{l}49 \\
(29-67)\end{array}$ \\
\hline $\begin{array}{l}\mathrm{lig} . \\
\text { LDFt }\end{array}$ & $\begin{array}{l}47 \\
(33-62)\end{array}$ & $\begin{array}{l}56 \\
(49-94)\end{array}$ & $\begin{array}{l}47 \\
(25-62)\end{array}$ & $\begin{array}{l}50 \\
(42-65)\end{array}$ & $\begin{array}{l}57 \\
(44-68)\end{array}$ & 57 & $\begin{array}{l}55 \\
(41-68)\end{array}$ & $\begin{array}{l}51 \\
(37-67)\end{array}$ \\
\hline unop. & 51 & 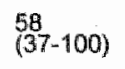 & $\begin{array}{l}62 \\
(45-89)\end{array}$ & $(340-82)$ & $\frac{62}{(49-87)}$ & $(38)$ & $\begin{array}{l}57 \\
(37-81)\end{array}$ & $\begin{array}{l}65 \\
(29-91)\end{array}$ \\
\hline $\begin{array}{l}\text { st } \\
\text { lig. }\end{array}$ & $\begin{array}{l}27.0 \\
(25.2 \\
29.0)\end{array}$ & $\begin{array}{l}25.7 \\
24.8\end{array}$ & $\begin{array}{l}26.1 \\
24.3 \mathrm{~m} \\
27.2)\end{array}$ & $\begin{array}{l}26.4 \\
25.2 \\
28.8\end{array}$ & $\begin{array}{l}26.1 \\
(24.6- \\
27.8)\end{array}$ & $\begin{array}{l}27.3 \\
(24.8 \\
28.6)\end{array}$ & $\begin{array}{l}25.1 \\
(24.5- \\
25.7)\end{array}$ & $\begin{array}{l}27.2 \\
(26.9 \\
27.7)\end{array}$ \\
\hline
\end{tabular}

Table 10.2: time course (days) of vasoconstrictor response to cold pressor test (LDFWr), basal skin blood flow (LDFb; in perfusion units), lowest skin blood flow value after the cold pressor test (LDFlv, in perfusion units). time to reach the lowest skin blood flow walue after the cold pressor test (LDFt, in sec.), and skin temperature $\left(\mathrm{ST}_{4}\right.$ in $\left.{ }^{\circ} \mathrm{C}\right)$; median values and interquartile ranges are presented for both the ligated (lig.) and the unoperated (unop.) group. 


\section{Nociceptive behavioural tests (table 10.3)}

When compared with preligation values, the duration of hind paw withdrawals from a chilled floor was increased in the ligated group (group I) from day 1 after ligation onwards $(p<0.01)$. After ligation, the duration of hind paw withdrawals did not change with time, apart from a decrease at day $21(\mathrm{p}<0.01)$. During the whole observation period no changes were observed in the duration of hind paw withdrawals on a chilled floor in the unoperated group (group II). When compared with preligation values, the duration of hind paw withdrawals on a warmed floor was increased $(\mathrm{p}<0.0001)$ in ligated rats (group I) from day 1 until day 7 . Thereafter, differences were no longer observed. During the whole observation period no changes were observed in the duration of hind paw withdrawals on a warmed floor in the unoperated group (group II). In group I, when compared with preligation values, 'spontaneous' pain-related behaviour was increased $(\mathrm{p}<0.0001)$ from day 1 after ligation onwards. In the unoperated group (group II), 'spontaneous' pain-related behaviour was never observed.

\begin{tabular}{|c|c|c|c|c|c|c|c|c|}
\hline & $\begin{array}{l}\text { initiall } \\
\text { values }\end{array}$ & 1 & 3 & 5 & 7 & 14 & 21 & 28 \\
\hline \multicolumn{9}{|c|}{ duration foot with-drawals chilled floor } \\
\hline lig. & $\begin{array}{l}0 \\
(0-9)\end{array}$ & $\begin{array}{l}8 \\
(0-14)\end{array}$ & $\begin{array}{l}21 \\
(10-30)\end{array}$ & $\begin{array}{l}13 \\
(6-26)\end{array}$ & $\begin{array}{l}15 \\
(5-30)\end{array}$ & $\begin{array}{l}15 \\
(10-30)\end{array}$ & $\begin{array}{l}5 \\
(2-7)\end{array}$ & $\begin{array}{l}19 \\
(9-30)\end{array}$ \\
\hline unop. & $\begin{array}{l}0 \\
(0-10)\end{array}$ & $\begin{array}{l}0 \\
(0-10)\end{array}$ & $\begin{array}{l}0 \\
(0-7)\end{array}$ & $\begin{array}{l}0 \\
(0-9)\end{array}$ & $\begin{array}{l}0 \\
(0-11)\end{array}$ & $\begin{array}{l}0 \\
(0-6)\end{array}$ & $\begin{array}{l}0 \\
(0-5)\end{array}$ & $\begin{array}{l}0 \\
(0-14)\end{array}$ \\
\hline \multicolumn{9}{|c|}{ duration foot withdrawals warmed floor } \\
\hline lig. & $\begin{array}{l}0 \\
(0-0)\end{array}$ & $\begin{array}{l}14 \\
(1-26)\end{array}$ & $\begin{array}{l}10 \\
(1-31)\end{array}$ & $\begin{array}{l}9 \\
(0-22)\end{array}$ & $\begin{array}{l}7 \\
(0-9)\end{array}$ & $\begin{array}{l}0 \\
(0-6)\end{array}$ & $\begin{array}{l}0 \\
(0-5)\end{array}$ & $\begin{array}{l}0 \\
(0-5)\end{array}$ \\
\hline unop. & $\begin{array}{l}0 \\
(0-0)\end{array}$ & $\begin{array}{l}0 \\
(0-0)\end{array}$ & $\begin{array}{l}0 \\
(0-0)\end{array}$ & $\begin{array}{l}0 \\
(0-0)\end{array}$ & $\begin{array}{l}0 \\
(0-0)\end{array}$ & $\begin{array}{l}0 \\
(0-0)\end{array}$ & $\begin{array}{l}0 \\
(0-0)\end{array}$ & $\begin{array}{l}0 \\
(0-0)\end{array}$ \\
\hline \multicolumn{9}{|c|}{ "spontaneous pain" - related behaviour } \\
\hline lig. & $\begin{array}{l}0 \\
(0-0)\end{array}$ & $\begin{array}{l}1.5 \\
(1.0- \\
2.2)\end{array}$ & $\begin{array}{l}2.0 \\
(1.0 \\
3.0)\end{array}$ & $\begin{array}{l}1.0 \\
(0.7- \\
2.0)\end{array}$ & $\begin{array}{l}1.5 \\
(1.0 \\
2.2)\end{array}$ & $\begin{array}{l}1.0 \\
(0.7- \\
2.0)\end{array}$ & $\begin{array}{l}2.0 \\
(1.0- \\
2.0)\end{array}$ & $\begin{array}{l}2.0 \\
(1.0-3.0)\end{array}$ \\
\hline unop... & $\begin{array}{l}0 \\
(0-0)\end{array}$ & $\begin{array}{l}0 \\
(0-0)\end{array}$ & $\begin{array}{l}0 \\
(0-0)\end{array}$ & $\begin{array}{l}0 \\
(0-0)\end{array}$ & $\begin{array}{l}0 \\
(0-0)\end{array}$ & $\begin{array}{l}0 \\
(0-0)\end{array}$ & $\begin{array}{l}0 \\
(0-0)\end{array}$ & $\begin{array}{l}0 \\
(0-0)\end{array}$ \\
\hline
\end{tabular}

Table 10.3: time course (days) of duration of foot withdrawals on chilled $\left(4^{\circ} \mathrm{C}\right)$ or warmed $\left(35^{\circ} \mathrm{C}\right)$ floor in seconds), muscle thickness (in $\mathrm{mm}$ ), and weight (in grams); median values and interquartile ranges are presented for both the ligated and the unoperated group. 


\section{DISCUSSION}

This study is the first to demonstrate that loose ligation of the seiatic nerve in rats induces an ongoing impairment of the vasoconstrictor response of skin microvessels to an autonomic provocation test (cold pressor test), which is suggestive of decreased sympathetic vasoconstrictor outflow. This decreased vasoconstrictor outflow may account for the increase in basal skin blood flow as observed at an early stage after ligation (day $3-5$ ). It is well known that an ongoingly deereased sympathetic outflow may induce supersensitivity of skin microvessels to catecholamines ${ }^{42}$. Hence, the decrease in skin blood flow as observed at a later stage after ligation (day $7-28$ ) may have been caused by supersensitivity of skin microvessels to (circulating) catecholamines rather than by an increased level of efferent sympathetic (vasoconstrictor) nerve impulses.

In rats subjected to loose ligation of a sciatic nerve, skin bllood flow was increased from day 3 until day 5 after ligation. An increase in skin blood flow may result from decreased sympathetic vasoconstrictor outflow ${ }^{314,510}$. Our finding that the sympathetically mediated vasoconstrictor response to the cold pressor test was consistently impaired from day 1 after loose ligation onwards argues in favour of this hypothesis. In the CCI model, this decreased sympathetic vasoconstrictor outflow may involve strictly functional inhibition of skin vasoconstrictor activity and/or decreased noradrenergic vasomotor innervation. Decreased noradrenergic vasomotor innervation, which has been detected in the CCI model previously ${ }^{533}$, may result from interruption of sympathetic efferents at the site of the actual nerve trauma. The hypothesis on interrupted sympathetic efferents is supported by the observation of axonal degeneration of small unmyelinated (most likely also sympathetic) nerve fibres in the loosely ligated sciatic nerve $\mathrm{e}^{31,69,208}$. In these studies, axonal degeneration of small unmyelinated nerve fibres varied from $20 \%{ }^{376}$ to as much as $84 \% \%^{69}$. However, the results of some studies suggest that besides acute interruption of sympathetic efferents other neuropathic mechanisms may be involved in the reduction of noradrenergic vasomotor innervation as observed in the $\mathrm{CCl}$ model. Wakisaka and colleagues ${ }^{533}$ demonstrated that (nearly) total noradrenergic depletion develops slowly over a period of about a month, whereas in the case of interruption of sympathetic efferents, one would expect that noradrenergic depletion develops more rapidly. Besides, it has been demonstrated that noxious stimulation of nociceptors (without damaging sympathetic nerve fibres) may also cause inhibition of skin vasoconstrictor activity ${ }^{200,237}$. In the $\mathrm{CCl}$ model, another mechanism involved in reduction of noradrenergic vasomotor innervation besides interruption of sympathetic efferents could be decreased synthesis of norepinephrine. The latter phenomenon has been demonstrated to result from both transection and crushing of a nerve $\mathrm{e}^{74,263}$.

In our study, we observed a consistently decreased sympathetic vasoconstrictor outflow, as indicated by the ongoing impairment of the vasoconstrictor response to the cold pressor test. It is well known that reduced release of a neurotransmitter may induce supersensitivity of effector cells to this transmitter ${ }^{36,42}$. In general, there is a delay in the appearance of supersensitivity, which, in vascular structures may last from 3 days up to $2-4$ weeks ${ }^{164}$. Hence, the increase in skin blood flow at an early stage after ligation, followed by a decrease at a later stage may result from decreased sympathetic vasoconstrictor outflow and consequent supersensitivity of skin microvessels to (circulating) catecholamines, respectively. The 
hypothesis that the decrease in skin blood flow at a later stage in the $\mathrm{CCl}$ model results from denervation-induced supersensitivity to catecholamines rather than from an increased level of efferent sympathetic (vasoconstrictor) nerve impulses is supported by the findings of Wakisaka et $\mathrm{a}^{535}$. These authors demonstrated in CCI rats that surgical sympathectomy of the affected limb does not have a significant effect on the lowered skin temperature as observed at two weeks after partial nerve injury.

In the unoperated rats, no significant changes were observed in mean skin blood flow or in the vasoconstrictor response to the cold pressor test during the whole observation period. These findings indicate that the skin blood flow abnormalities in the ligated group indeed result from loose ligation of the ipsilateral sciatic nerve. Besides, these findings indicate that in our study repeated laser Doppler flow measurements and increasing age/weight did not have any effect on skin blood flow.

On the basis of the findings in our study, we can not exclude the possibility that an an tidromically acting $\mathrm{C}$-nociceptor system ${ }^{392}$ is also involved in the increase in skin blood flow from day 3 until day 5 after ligation. The vasodilator action of such an antidromically acting $\mathrm{C}$-nociceptor system is mediated by release of vasodilator neuropeptides at the periphery of small unmyelinated $\mathrm{C}$ nociceptor nerve fibres consequent to excitation of these fibres at the midaxon level ${ }^{318.460}$. In the CCI model, mechanical or chemical excitation of nociceptor nerve fibres at midaxon level may result from the constrictive procedure itself or from formation of granulation tissue. Besides, on the basis of the findings in our present study we can also not exclude that the increase in skin blood flow from day 3 until day 5 after ligation is mediated by activation of (vasodilator) $B$-adrenoceptors. However, so far no direct evidence for the existence of $\beta$-adrenoceptors in rat skin is available, which argues against this hypothesis.

At day 3,95\% of all rats demonstrated an abnormal posture of the hind paw, whereas in the unoperated animals this abnormal posture was never observed. This finding indicates that in our study loose ligation of the sciatic nerve almost invariably caused neuropathy. However, the rapidity with which trophic changes of muscles and claws as well as behavioural changes indicative of neuropathic pain developed differed considerably between the rats. This heterogeneity may have been caused by intra-individual differences in tightness of ligation.

Thermography consistently measures thermal changes and delineates the areas of such thermal changes. Nevertheless, the results on thermography as found in our study as well as in another study in the CCI model ${ }^{40}$ did not relate to the observed pattern of changes in skin blood flow. This finding suggests that thermography is not an adequate index of skin microcirculation, as has also been demonstrated by Tooke et al ${ }^{512}$. These authors showed that skin blood flow can change significantly without any measurable change in skin temperature.

In our study, we observed a rather homogeneous pattern of changes in skin blood flow which did not relate to changes in skin temperature. A previous study in the $\mathrm{CCI}$ model also did not demonstrate significant changes in skin temperature with time ${ }^{40}$. In contrast, other thermographic studies demonstrated that skin temperature in the $\mathrm{CCl}$ model evolves from an increase at an early stage to a decrease at later stages ${ }^{39,533}$, although the results of thermography in these studies were less homogeneous than the results of laser Doppler flowmetry. This discrepancy may relate to the fact that skin temperature is not only influenced by local skin blood flow but also by other factors such as the local metabolic rate. 
In rats which had not yet been subjected to loose ligation of a sciatic nerve, exposure to a chilled floor $\left(4^{\circ} \mathrm{C}\right)$ evoked hind paw withdrawals in some $(25 \%)$ of the rats, whereas exposure to a warmed floor $\left(35^{\circ} \mathrm{C}\right)$ did not evoke hind paw withdrawals at all. These findings indicate that thermal stimulation at $4^{\circ} \mathrm{C}$ but not at $35^{\circ} \mathrm{C}$ may stimulate nociceptors, even in the absence of neuropathy. Hence, increased duration of hind paw withdrawals following stimulation at $35^{\circ} \mathrm{C}$ reflects allodynia. In contrast, increased duration of hind paw withdrawals following thermal stimulation at $4^{\circ} \mathrm{C}$ does not seem to reflect allodynia as has been suggested previously ${ }^{40,212}$, but more likely cold-stress-induced ongoing pain ${ }^{80}$. The different nature of these stimulus conditions (at $4{ }^{\circ} \mathrm{C}$ or $35^{\circ} \mathrm{C}$ ) may also account for the observed differences in time course of nociceptive behaviour.

Muscle thickness was significantly decreased from day 3 after ligation onwards. These atrophic changes may be related not only to disuse of the affected hind paw, but also to the observed reduction of blood flow. 


\section{Chapter 11}

SKIN BLOOD FLOW DISTURBANCES

IN THE CONTRALATERAL LIMB

IN A RAT MODEL OF PARTIAL NERVE INJURY

HAJM Kurvers, GJ Tangelder, JGR De Mey, DW Slaaf, FAJM van den Wildenberg, PJEHM Kitslaar, RS Reneman, EV Rouwet, and MJHM Jacobs

Departments of Surgery of the University Hospital Maastricht and of the Academic Medical Centre Amsterdam, The Netherlands.

Departments of Surgery, Physiology, Pharmacology, and Biophysics of the Cardiovascular Research Institute Maastricht (CARIM), The Netherlands.

Based on: Neuroscience, 1996, vol. 74 (3), p. 935-943. 


\section{SUMMARY}

Electrical excitation of nociceptive afferents in an extremity has been demonstrated to increase skin blood flow in the contralateral extremity. Hence, one would expect that loose sciatic nerve ligation, which induces an experimental painful peripheral neuropathy, may also provoke a vasodilator response in the contralateral hind paw. On the nonligated side, such a response may involve inhibited skin vasoconstrictor activity as well as neurogenically mediated active vasodilation. We studied skin blood flow changes in the rat hind paw consequent to contralateral loose sciatic nerve ligation. After ligation, we additionally investigated whether blockade of afferent input from the ligated sciatic nerve to the spinal cord, by means of lidocaine, overrules the vasodilator response in the nonligated paw. On the nonligated side, we assessed the vasoconstrictor response of skin microvessels to cooling of the rat abdomen as a measure of skin vasoconstrictor activity. in this paw. In order to investigate the involvement of sensory and/or nonsensory nerve fibres in the nonligated sciatic nerve on skin blood flow abnormalities in the nonligated paw, we studied the influence of blockade of these fibres through successive capsaicin and lidocaine application. We show that loose ligation of the sciatic nerve induces a vasodilator response in the contralateral hind paw, which is completely abolished by blockade of afferent input from the ligated sciatic nerve. From day 1 after ligation, skin vasoconstrictor activity in the nonligated paw was reduced, as indicated by an impaired vasoconstrictor response to cooling of the rat abdomen. Besides, blockade of sensory but not of nonsensory nerve fibres on the nonligated side attenuated the vasodilator response in this paw. The data presented here indicate that loose ligation of the rat sciatic nerve induces a vasodilator response in the contralateral hind paw. On the nonligated side, this vasodilator response may involve inhibition of skin vasoconstrictor activity as well as antidromically acting sensory nerve fibres.

\section{INTRODUCTION}

Blumberg and Wallin have demonstrated that in humans painful intraneural electrical stimulation increases skin blood flow not only ipsilaterally, but also in the contralateral extremity ${ }^{53}$. Since the vasodilator response in the nonstimulated extremity occurred only when stimulation strength was high enough to cause sharp and localised pain, the authors proposed that the afferent limb of this reflex involves excitation of A- $\delta$ fibres. Likewise, Loven ${ }^{738}$ demonstrated that in rabbits electrical stinulation of cutaneous merves induces dilation of cutaneous vessels in the contralateral extremity.

Loose ligation of a sciatic nerve in rats (chronic constriction injury (CCI)) induces an experimental painful peripheral neuropathy that reproduces many of the signs and symptoms observed in clinical conditions of neuropathic origin such as causalgia and reflex sympathetic dystrophy ${ }^{39,40,392,557}$. Additionally, this nerve constriction injury has been demonstrated to cause excitation of nociceptive afferents $^{260.62}$. Hence, one would expect that loose sciatic nerve ligation may also induce a vasodilator response in the contralateral hind paw. Such a phenomenon would render this paw unfit to serve as control for studies on changes in skin blood flow and skin temperature on the ligated side ${ }^{39,533}$. Therefore, we measured skin blood flow in the rat hind paw, before and after contralateral loose sciatic nerve ligation. To test the hypothesis that skin blood flow abnormalities in the rat hind paw induced by contralateral loose sciatic nerve ligation, indeed result from increased afferent input from the loosely ligated sciatic nerve to the spinal cord, we studied the influence of blockade of the loosely ligated sciatic nerve, by means of lidocaine, on skin blood flow in the nonligated hind paw.

A vasodilator response on the nonligated side may result from reduced (sympathetic) skin vasoconstrictor activity in this paw. It has been demonstrated previously that actiwation of nociceptive afferents may reduce skin vasoconstrictor activity ${ }^{200.237,25 \%}$. Therefore, we assessed on the nonligated 
side, as an indirect measure of skin vasoconstrictor activity, the effectiveness of the vasoconstrictor response of skin microvessels to cooling of the rat abdomen. It has been demonstrated by Linderoth and colleagues ${ }^{328}$ that this vasoconstrictor response is impaired in case of reduced (sympathetic) skin vasoconstrictor activity.

On the nonligated side, a neurogenically mediated vasodilator response may involve an tidromically acting sensory ( $\mathrm{C}$ and $\mathrm{A}-\delta$ ) and/or orthodromically acting sympathetic nerve fibres. This consideration prompted us to examine the influence of blockades of both types of nerve fibres in the nonligated sciatic nerve on the vasodilator response in the nonligated hind paw. Firstly, the capability of sensory ( $\mathrm{C}$-nociceptor and $\mathrm{A}-\mathrm{\delta}$ ) nerve fibres to mediate a neurogenic inflammatory (vasodilator) response was blocked by means of perineural application of capsaicin ${ }^{85,662,256,317}$. Thus, if capsaicin would attenuate a vasodilator response induced by contralateral loose sciatic nerve ligation it is likely that sensory nerve fibres in the nonligated sciatic nerve are involved in this response. Following capsaicin application, impulse propagation of non-sensory nerve fibres was blocked by means of lidocaine to explore the involvement of these fibres in the vasodilator response induced by contralateral loose sciatic nerve ligation. Since so far no evidence has been presented for the involvement of other than antidromically acting sensory or orthodromic acting sympathetic nerve fibres in neurogenic skin wasomotor control, the effects of lidocaine application to capsaicin pretreated nerves on skin blood flow can most likely be attributed to blockade of sympathetic nerve fibres.

\section{MATERIALS AND METHODS (for detailed information see chapter 4)}

\section{Materials}

Of the 52 male Lewis rats used, 44 were subjected to loose ligation of the right sciatic nerve. The remaining 8 rats were used to obtain information on the effects on skin blood flow of capsaicin and lidocaine application to non-ligated sciatic nerves.

\section{Experimental protocol}

In a first group of 20 rats (group I) laser Doppler flowmetry measurements were performed one day before, as well as $1,3,5,7,14,21$, and 28 days after ligation of the contralateral sciatic nerve. This protocol was chosen in order to assess the time course of changes in skin blood flow (SBF) induced by contralateral loose sciatic nerve ligation. At these timepoints, skin blood flow was measured. Subsequently, the abdomen of the rat was cooled by spraying ethylchloride (cold pressor test, CPT). After the CPT skin blood flow was again measured. The ratio of mean skin blood flow before the $\mathrm{CPT}$ divided by the lowest value after the CPT was calculated to evaluate the effectiveness of the skin vasoconstrictor response. A ratio lower than that found in the preligation situation is indicative of reduced skin vasoconstrictor activity ${ }^{328}$.

In a second group of 8 rats (group II) we measured SBF on the nonligated side one day before contralateral loose sciatic nerve ligation as well as 5 days after this procedure. At day 5 after ligation, SBF was measured on the nonligated side before and after exposure of the (contralateral) loosely ligated sciatic nerve. Subsequently, lidocaine $2 \%$ was applied to the loosely ligated sciatic nerve in order to block afferent input from the sciatic nerve to the spinal cord ${ }^{205}$. SBF was again determined 10 and 60 minutes later.

In a third group of 8 rats (group III), SBF was measured on the nonligated side one day before contralateral loose sciatic nerve ligation as well as 5 days after this procedure. At day 5 after ligation SBF measurements were performed on the nonligated side before and after exposure of the (ipsilateral) nonligated sciatic nerve. Following exposure of the nonligated sciatic nerve, the capability of sensory 
fibres in this nerve to mediate a neurogenic inflammatory (vasodilator) response was abolished by means of perineural capsaicin application. SBF wass again assessed at 10 and 60 minutes following the capsaicin application.

Following this one hour observation period (during which the influence of capsaicin application on SBF was assessed), we applied lidocaine to the capsaicin-pretreated nonligated sciatic nerve to block impulse propagation of nonsensory nerve fibres. Since so far no evidence has been presented for the involvement of other than sensory and sympathetic nerve fibres in neurogenic skin vasomotor control, the effect of lidocaine application to capsaicin pretreated nerves on skin blood flow abnormalities most likely can be attributed to blockade of impulse propagation of sympathetic nerve fibres. SBF was then again determined 10 and 60 minutes later.

In a fourth group of 8 rats (group IV), LDF was employed to obtain information on systemic effects of lidocaine application on SBF abnormalities induced by contralateral loose sciatic nerve ligation. To this end; SBF was assessed in the nonligated hind paw before ligation and at day 5 after ligation. At day $5, \mathrm{SBF}$ was assessed before and after creation of a surgical wound in the left fore paw of the rats. To this end, the triceps brachii muscle was dissected bluntly. The size of this surgical wound in the fore paw was comparable to the size of the surgical wound in the hind paw used for the application of capsaicin and lidocaine to the sciatic nerve. Lidocaine was then applied in such a way that a layer of the solution was continuously present in this wound. SBF measurements in the nonligated hind paw were repeated 10 and 60 minutes later.

In another group of 8 rats (group V), LDF was employed to obtain information on the effects on SBF of capsaicin and lidocaine application to nonligated sciatic nerves. To this end, capsaicin and lidocaine were applied consecutively, as described above. SBF was measured before and after exposure of the sciatic nerve. Following exposure, SBF was measured at 10 and 60 minutes following the 15 minutes capsaicin application, and at 10 and 60 mimutes after the start of subsequent lidocaine application.

\section{RESULTS}

Fig. $\mathbb{1 1 . 1}$ shows that in group $\mathbb{I}$, when compared with preligation values (normalised value $=\mathbb{1}$, interquartile range absolute SBF values $50-120$ perfusion units) loose ligation of the sciatic nerve induced an increase in SBF on the nonligated side from day 3 after ligation onwards $(\mathrm{p}<0.001)$, stabilising at a plateau after day 7 .

Fig. 11.2 shows that in group I, when compared with preligation values (normalised value $=1$, interquartile range absolute values $1.49-2.38)$, loose ligation of the right sciatic nerve induced $(\mathrm{p}<0.01)$ an impairment of the vasoconstrictor response to the cold pressor test from day 3 onwards, with the exception of day 14 .

Fig. 11.3 demonstrates that in group II, when compared with preligation values (normalised value $=1$, interquartile range absolute $\mathrm{SBF}$ values $48-116$ perfusion units), $\mathrm{SBF}$ on the nonligated side was increased at day $5(3.51, \mathrm{p}<0.01)$. Exposure of the loosely ligated sciatic nerve did not change contralateral SBF (3.04). Subsequent blockade of the afferent input from the loosely ligated sciatic nerve to the spinal cord by means of lidocaine resulted in a decrease in SBF on the nonligated side. As compared to postexposure values, SBF was reduced both at $10(1.44, \mathrm{p}<0.01)$ and 60 minutes $(1.32$, $\mathrm{p}<0.01)$ after the start of the lidocaine application. Moreover, at both time intervals, SBF values did not differ anymore from preligation values. 


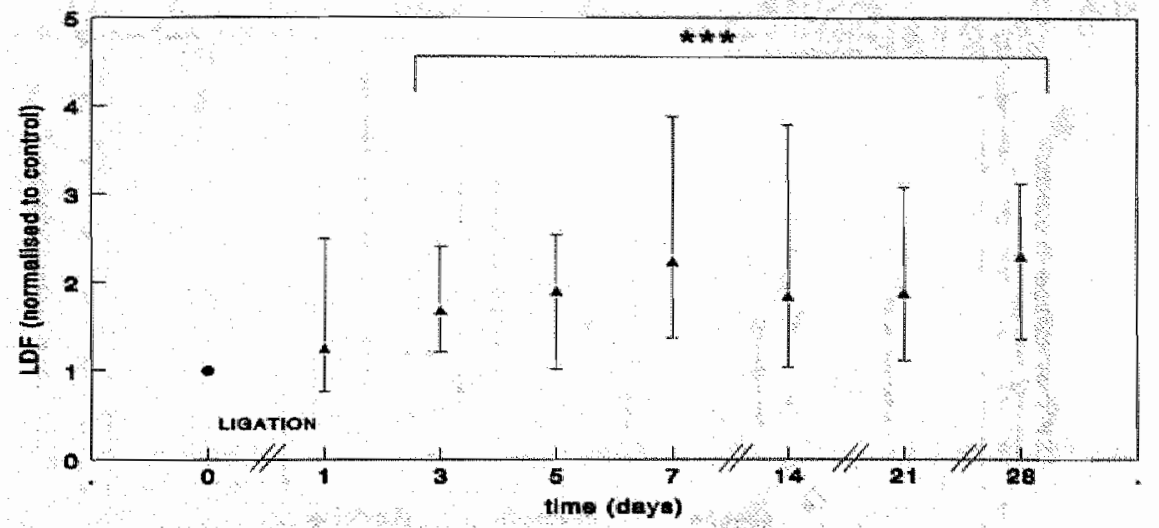

Fig. 11.1: LDF values as measured in the nonligated hind paw before loose ligation of the sciatic nerve in the other hind paw as well as at several timepoints after this procedure. All data are nomalised to preligation values $(=1$ ), and presented as medians (dots) and interquartile ranges (error bars), On the Y-axis SBF. On the $X$-axis the different timepoints. **: $p<0.001$ as compared to preligation SBF values.

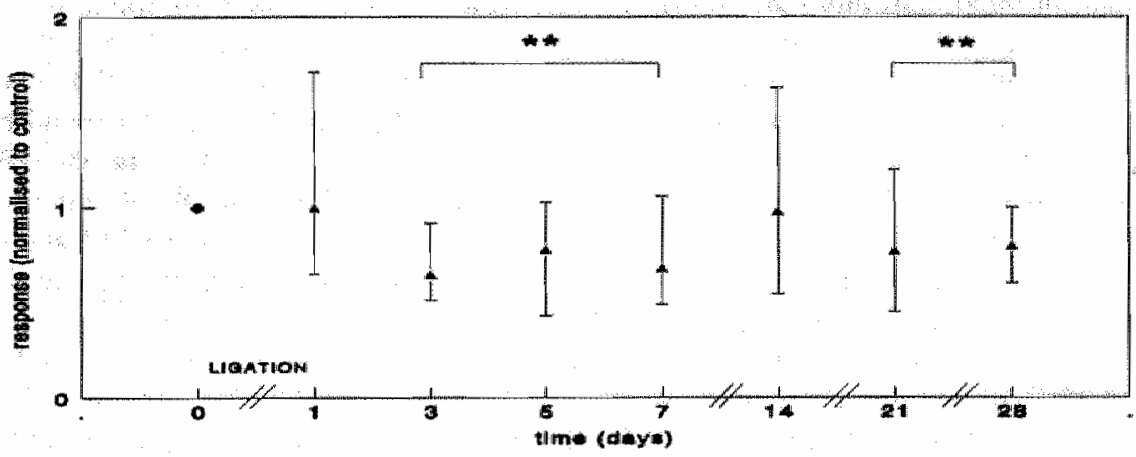

Fig. 11.2: vasoconstrictor response to cold pressor test of skin microvessels as measured in the nonligated hind paw before loose ligation of the sciatic nerve in the other hind paw as well as at several timepoints after this procedure. All data are normalised to preligation values (=1), and presented as medians (dots) and interquartile ranges (error bars). On the $Y$-axis the vasoconstrictor response. On the $X$-axis the different timepoints. $p<0.01$, as compared to preligation values. 


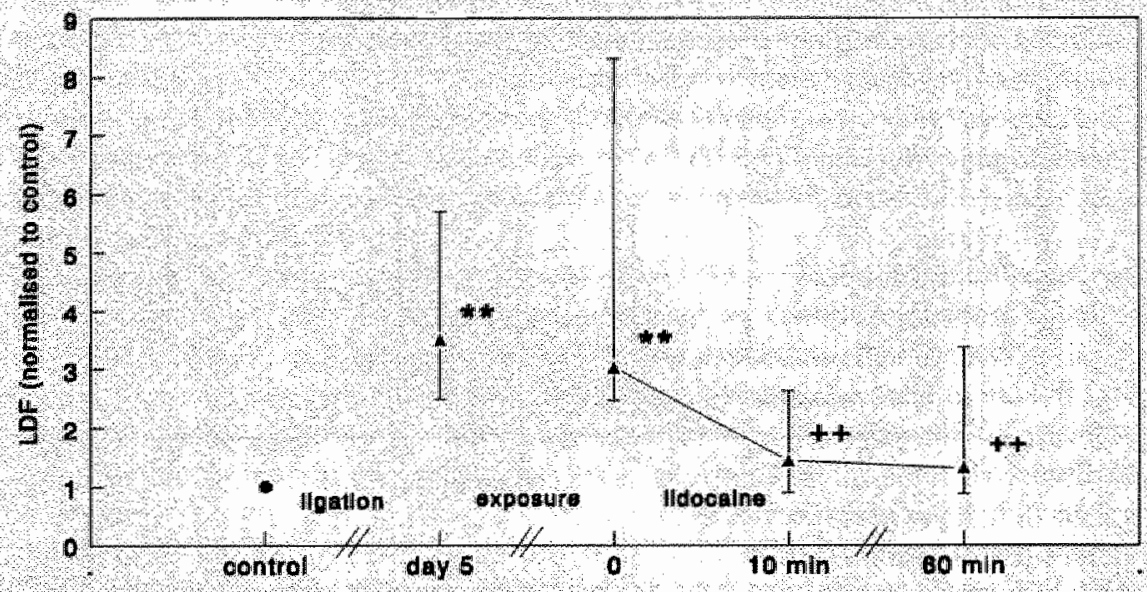

Fig. 11.3. LDF values as measured in the nonligated hind paw before ligation and at day 5 . At this timepoint: before and after exposure of the loosely ligated sciatic nerve and at 10 and 60 minutes after blockade of afferent input from the ligated sciatic nerve to the spinal cord (designated lidacaine). All data are normalised to preligation values $(=1)$, and presented as medians (dots) and interquartile ranges (error bars). On the $Y$-axis SBF On the $X$-axis the different timepoints. **: $p<0.01$, as compared to values obtained before ligation, ++: $p<0.01$, as compared to values obtained after exposure of the nonligated sciatic nerve.

Fig. 11.4 shows that in group III, when compared with preligation values (normalised value $=$ interquartile range absolute SBF values $27-75$ perfusion units), $\mathrm{SBF}$ on the nonligated side wa increased at day $5(3.32, p<0.05)$. Exposure of the nonligated sciatic nerve did not change ipsilater SBF (3.83). Subsequent application of capsaicin to the nonligated sciatic nerve resulted in a temporat increase in ipsilateral SBF immediately upon application (data not shown). This increase, howeve reached a maximum within several minutes after the removal of the capsaicin solution. Thereafter, th initial increase was followed by a decrease in ipsilateral SBF. As a result, when compared wi postexposure values, SBF was decreased following capsaicin application, being 2.81 at 10 minutes $<0.05)$ and 2.77 at 60 minutes $(p<0.05)$. Nevertheless, SBF values remained increased at $10(p<0.0$ and 60 minutes $(p<0.05)$ when compared with preligation values.

Fig. 11.4 also demonstrates that subsequent application of lidocaine did not influence SBI being 2.38 at 10 minutes and 2.50 at 60 minutes. As a result, SBF values remained increased at 1 minutes $(p<0.05)$ and at 60 minutes $(p<0.05)$, when compared with preligation values.

In group $\mathbb{V}$, when compared with preligation values (normalised value $=1$, interquartile rang absolute SBF values 25-79 perfusion units) SBF on the nonligated side was increased at day 5 (3.5. $\mathrm{p}<0.05$ ). Creation of a surgical wound in the fore paw did not influence SBF vallues $(2.85)$ in th nonligated hind paw. As compared to the values obtained after creation of this surgical woun subsequent application of lidocaine into the surgical wound did not change SBF in the nonligated hin paw, being 3.02 at 10 minutes and 1.89 at 60 minutes after the lidocaine application. 


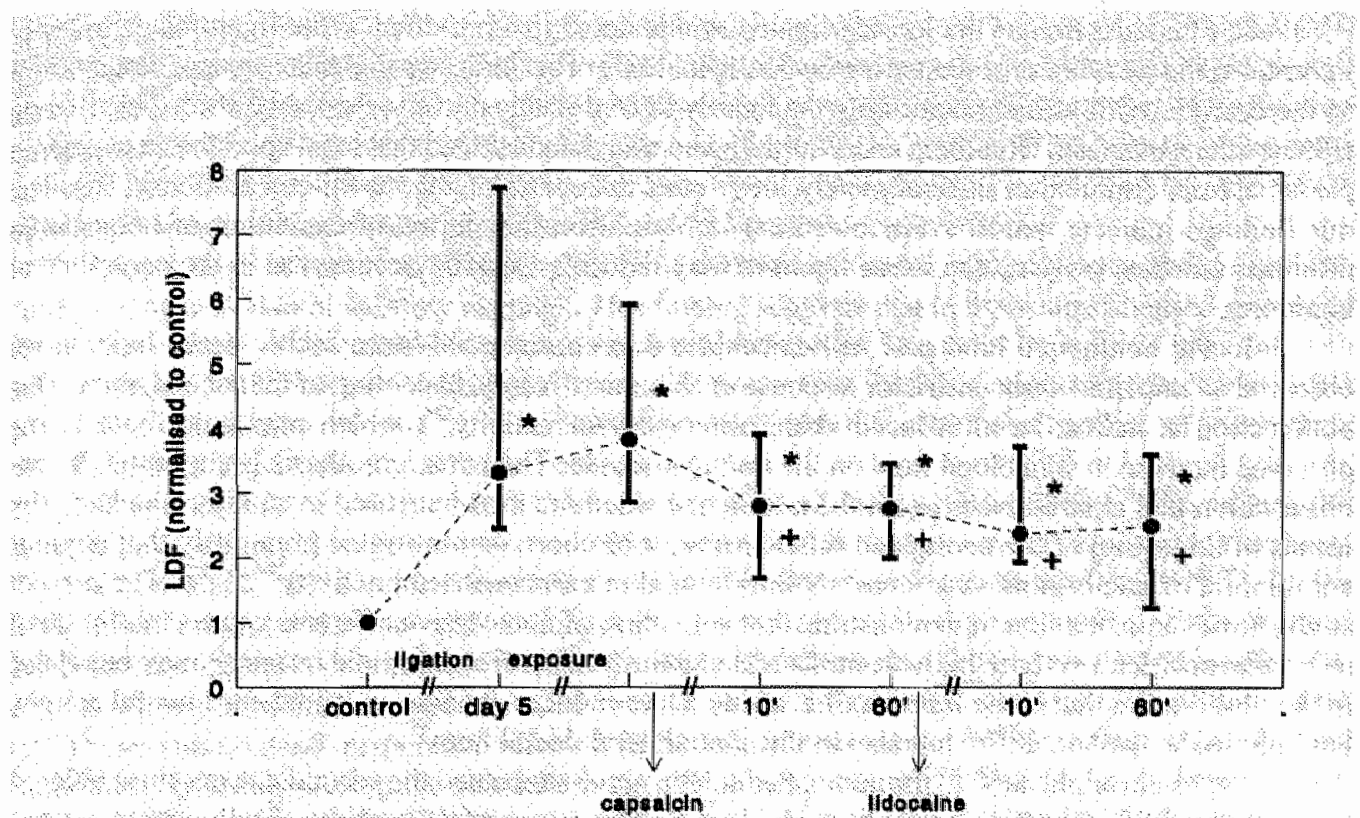

Fig. 11.4: LBE values as measured in rat skin before ligation and at day 5 . At this timepoint before and after exposure of the sciatic nerve, at 10 and 60 minules after blockade of Cnociceptor nerve fibres (designated capsalcin, and at 10 and 60 minutes after blockade of conduction of other than $C$-nociceptor nerve fibres (designated lidocaine) Al data are nomalised to preligation values $(=1$ ), and presented as medians (cots) and interquartile ranges (erior bars) On the $Y$ axis SBF. On the X axis the different time points * $p<0.05$. as compared to values obtained before ligation? $+\mathrm{p}<0.05$ as compared to values obtained after exposure of the nonligated sciatic nerve.

In group V (nonligated rats), SBF before exposure of the sciatic nerve (normalised value $=1$, interquartile range absolute SBF values 26 - 48 perfusion units), did not differ from postexposure values (0.86). As was the case in group III, subsequent application of capsaicin to the sciatic nerve resulted in a temporary increase in ipsilateral SBF immediately upon application (data not shown). This increase again reached a maximum within several minutes after the removal of the capsaicin solution. Thereafter, the initial increase in $\mathrm{SBF}$ subsided, as a result of which $\mathrm{SBF}^{\mathrm{F}}$ did not differ from postexposure values, being 1.0 at 10 minutes and 1.23 at 60 minutes after capsaicin application. After subsequent lidocaine application, $\mathrm{SBF}$ also did not differ from postexposure values, being 0.95 at 10 minutes and 1.09 at 60 minutes after lidocaine application.

\section{DISCUSSION}

This study shows that loose ligation of the rat sciatic nerve induces an increase in skin blood flow in the contralateral hind paw from day 3 after ligation onwards. Blockade of afferent input from the loosely ligated sciatic nerve to the spinal cord completely interrupts this vasodilator response. On the nonligated side, the vasodilator response may involve inhibited skin vasoconstrictor activity as well as release of vasodilator neuropeptides at the periphery of antidromically acting sensory nerve fibres. 
Blockade of conduction of the loosely ligated sciatic nerve just proximal to the ligatures completely intemupted the vasodilator response on the nonligated side. This indicates that this response results from an increased level of afferent input from the loosely ligated sciatic nerve to the spinal cord. This finding tallies with results from Blumberg and Wallin ${ }^{53}$, who also demonstrated that local anaesthesia of a nerve proximal to its stimulation site completely internupted the contralateral vasodilator tesponse. Besides, our tindings indicate that not only electrical ${ }^{53,338}$ but also other forms of excitation of nociceptive afferents (such as loose sciatic nerve ligation) may induce a wasodilator response in the contralateral extremity.

In the nonligated hind paw of rats subjected to contralateral loose sciatic nerve ligation we observed an impaired vassoconstrictor response of skin microvessels to cooling of the rat abdomen. This observation is indicative of reduced skin vasoconstrictor activity ${ }^{328}$, which may contribute to the observed increase in skin blood flow on the nonligated side. The latter hypothesis is supported by our observation that skin blood flow on the nonligated side remained increased even after blockade (by means of lidocaine) of the nonligated sciatic nerve. It has been demonstrated previously that noxious stimulation of nociceptors may cause inhibition of skin vasoconstrictor activity ${ }^{200,237,252}$. The present study, however, is the first to demonstrate that activation of nociceptive afferents causes inhibition of skin wasoconstrictor activity in the contralateral extremity. This crossed spinal response may be related to the observation that loose ligation of a sciatic nerve induces changes in spinal cord neural activity not only in the ipsilateral ${ }^{38534}$ but also in the contralateral dorsal horn ${ }^{347}$.

Capsaicin application to the nonligated sciatic nerve attenuated the vasodilator response induced by contralateral loose sciatic nerve ligation. This finding suggests that antidromically acting sensory nerve fibres in the nonligated sciatic nerve are involved in this vasodilator response. Such a neurogenically mediated vasodilator response may involve antidromically acting unmyelinated (C) as well as small myelinated (A- $\delta$ ) sensory nerve fibres ${ }^{255}$. Subsequent application of lidocaine to the nonligated sciatic nerve, in which the capability of sensory nerve fibres to mediate a neurogenic inflammatory (vasodilator) response had been blocked by capsaicin, did not influence the vasodilator response induced by contralateral loose sciatic nerve ligation. Hence, on the nonligated side this response does not seem to involve other than sensory nerve fibres.

As alluded to before, excitation of primary afferent sensory neurons may provoke release of vasodilator neuropeptides from their peripheral terminals $174,255,318,460$. Hence, a third mechanism which may account for the observed increase in skin blood flow on the nonligated side could be circulation of these neuropepticles from the ligated to the nonligated extremity. In our study we observed that interruption of afferent input from the injured sciatic nerve to the spinal cord by means of lidocaine application completely interrupted the contralateral vasodilator response. This finding seemingly argues in favour of neural mediation across the spinal cord. Nevertheless, one should realise that lidocaine application to the injured sciatic nerve may simultaneously block release of vasodilator neuropeptides from its peripheral terminals. Blockade of sensory nerve fibres in the nonligated sciatic nerwe (through application of capsaicin) attenwated the vasodilator response on the nonligated side, which suggests that this response is at least partly neurally mediated. However, given the observation that skin blood flow on the nonligated side remained increased even after complete blockade of the nonligated sciatic nerve (through application of lidocaine), we can not exclude that a humoral component is also involved in the development of the contralateral vasodilator response. In line with the findings of our study Levine et al $^{321}$ demonstrated that excitation of primary afferent sensory neurons, following unilateral hind limb injury, provokes an acute inflammatory response in the contralateral hind paw: Interruption of venous circulation of the injured limb did not alter the contralateral response, whereas acute denervation of either limb significantly attenuated the contralateral response. These findings argue against the hypothesis of humoral mediation of contralateral inflammatory responses. 
In both ligated and nonligated rats we observed a transient increase in ipsilateral skin blood flow immediately upon application of capsaicin to the nonligated sciatic nerves which reached its maximum a few minutes after the removal of the piece of cotton containing the capsaicin solution. This transient ipsilateral increase, which has been described previously by Gamse and Sarial ${ }^{174}$, is likely to represent the acute excitation phase during which an antidromic barrage is created as a result of which vasodilator neuropeptides are released from the terminals of primary afferent nerve fibres.

Application of capsaicin as well as lidocaine to the sciatic nerve of nonligated rats did not cause lasting changes in skin blood flow readings. This finding suggests that in these anaesthetised rats blood flow in the cutaneous vasculature is not neurogenically controlled. The latter hypothesis is in line with the findings of Willette and colleagues ${ }^{556}$, who demonstrated that the vasoconstrictor tone in the cutaneous vasculature of rats is predominantly mediated by a humoral action of circulating catecholamines at postjunctional $\alpha$-adrenoceptors.

Loose ligation of a sciatic nerve in rats, which activates nociceptive afferent $s^{260,562}$, induces an increase in skin blood flow in the contralateral extremity. Other studies have demonstrated that loose ligation of a sciatic nerve induces sensory abnormalities in the contralateral hind paw ${ }^{23,280,474}$. In line with these findings, Levine and colleagues ${ }^{321}$ demonstrated that a standard injury stimulus (subcutaneous injection of normal saline into the foot pad), which also activates nociceptive afferents, induces sensory abnormalities and increased vascular permeability in the contralateral hind limb of the rat. These contralateral responses, referred to as reflex neurogenic inflammation, also involved antidromically acting nociceptive afferents in the noninjured extremity ${ }^{32 !}$. The similarity between their and our findings suggests that similar mechanisms are involved in these contratateral responses.

In line with our findings in this experimental animal model of neuropathic pain, we demonstrated skin blood flow abnormalities in the clinically nonaffected extremities of RSD patients ${ }^{301}$. This congruity further attest to the usefumess of the CCI model in the evaluation of pathophysiological mechanisms underlying RSD.

In conclusion, the present study shows that loose ligation of the rat sciatic nerve induces an increase in skin blood flow in the contralateral hind paw from day 3 after ligation onwards. Blockade of afferent input from the loosely ligated sciatic nerve to the spinal cord interrupts this vasodilator response. On the nonligated side the vasodilator response may involve inhibited skin vasoconstrictor activity as well as release of vasodilator neuropeptides at the periphery of antidromically acting sensory nerve fibres. The resuits of these experiments may have important implications for the practice of using one extremity as a control for studies on skin blood flow, and probably also skin temperature, on the (contralateral) ligated side. 


\section{Chapter 12}

PARTIAL NERVE INJURY IN THE RAT:

INFLUENCE ON EFFERENT FUNCTION OF SYMPATHETIC AND ANTIDROMICALLY ACTING SENSORY NERVE FIBRES

HAJM Kurvers, GJ Tangelder, JGR De Mey, RS Reneman, DW Slaaf, EV Rouwet, FAJM van den Wildenberg, PJEHM Kitslaar, MJHM Jacobs.

Departments of Surgery of the University Hospital Maastricht and Academic Medical Centre Amsterdam, The Netherlands.

Departments of Surgery, Physiology, Pharmacology, and Biophysics of the Cardiovascular Research Institute Maastricht (CARIM), The Netherlands.

Based on: Journal of Trauma, 1996, vol. 41, no. 1, p. 981-988 


\section{SUMMARY}

Following loose ligation of a sciatic nerve, rats develop signs and symptoms alike those observed in causalgia and reflex sympathetic dystrophy such as behavioural signs indicative of pain, trophic changes of muscles and claws, and abnormal skin temperature. Various studies have demonstrated increased afferent activity of spared sensory nerves following this type of nerve injury. In contrast, little is known about the effects of partial nerve injury on efferent (vasomotor) function of affected nerve fibres. The presence of skin temperature abnormalities suggests that this function is altered. Mostly, this constrictive procedure has been purported to evoke an increased level of efferent sympathetic nerve impulses. Besides altering efferent sympathetic nerve activity, it has been suggested that this type of nerve injury may also alter efferent activity of sensory nerve fibres; antidromic impulses may arise in sensory fibres as a result of which vasodilator neuropeptides may be released at the peripheral endings of these nerve fibres. In rats, skin blood flow may be influenced by excitation of both sympathetic and antidromically acting sensory nerve fibres. Consequently, skin blood flow may be used as a measure of efferent (vasomotor) function of both types of nerve fibres. We assessed, by means of laser Doppler flowmetry, skin blood flow (SBF) in the hind paw of male Lewis rats before, at an early (day 4), and at a later stage (day 21) after partial injury of the ipsilateral sciatic nerve (through loose ligation). This procedure has been reported to induce signs and symptoms alike those observed in patients with causalgia. At the two time-points after nerve injury, SBF was assessed before and after (chemical) blockade of sensory and non-sensory (sympathetic) sciatic nerve fibres. At day 4, as compared to preligation values, we observed an increase in SBF which was reduced by blockade of sensory nerve fibres. Subsequent blockade of non-sensory nerve fibres further reduced SBF. At day 21, SBF was decreased as compared to preligation values. Blockade of sensory nerve fibres further reduced $S B F$, and subsequent blockade of non-sensory nerve fibres did so as well. Partial injury of the rat sciatic nerve causes an ipsilateral increase in SBF at an early stage, which is followed by a decrease at a later stage. At both stages antidromically acting sensory and orthodromically acting non-sensory (sympathetic) nerve fibres are involved in the vasodilator response. At a later stage, however, neurogenic vasodilator mechanisms are overruled by a non-neurogenic vasoconstrictor mechanism. The latter may consist of supersensitivity of skin microvessels to catecholamines consequent to reduced neurogenic disposition of catecholamines.

\section{INTRODUCTION}

Causalgia is a pain syndrome which follows partial injury to a peripheral nerve trunk ${ }^{419}$. The syndrome is characterised by 1) sensory, 2) motor, 3) trophic, and 4) autonomic disturbances ${ }^{460}$. The most troubling feature for most patients is severe burning pain. Autonomic disturbances are among others reflected by an increase in skin blood flow at an early stage after nerve injury which develops to a decrease at later stages ${ }^{298,299}$. These skin blood flow abnormalities most likely account for the clinical observation that, in most causalgia patients, colour and temperature of the skin evolve from red $^{313}$ and warm ${ }^{355}$ at an early stage to cool and pale $\mathrm{e}^{115}$ at later stages. 
In 1988, Bennett and $\mathrm{Xie}^{40}$ reported on an animal model of partial peripheral nerve injury (chronic constriction injury (CCI)) in which many signs and symptoms alike those observed in causalgia could be induced $39,40,392,507$. Following nerve injury, these rats develop 1) behavioural signs indicative of spontaneous pain, hyperaigesia, and allodynia ${ }^{23}$, 2) abnormal gait and posture ${ }^{23}, 3$ ) trophic changes of muscles and claws ${ }^{40}$, and 4) abnormal skin temperature 39.40.

Various studies have demonstrated increased afferent activity of spared sensory nerves following this type of nerve injury ${ }^{261,562}$. In contrast, little is known about the effects of partial nerve injury on efferent (vasomotor) function of affected nerve fibres. The presenee of skin temperature abnormalities suggests that this function is altered. Mostly, this constrictive procedure has been purported to evoke an increased level of efferent sympathetic nerve impulses because surgical and chemical sympathectomy may relieve sensory abnormalities and increase skin temperature ${ }^{39,281,382}$. However, some recent studies have presented evidence of autonomic denervation in the affected limbs of $\mathrm{CCl}$ rats ${ }^{532,533}$. Besides altering efferent sympathetic nerve activity, it has been suggested that this type of nerve injury may also alter efferent activity of sensory nerve fibres; antidromic impulses may arise in sensory fibres as a result of which vasodilator neuropeptides may be released at the peripheral endings of these nerve fibres ${ }^{32}$. The latter phenomenon has been referred to as antidromic vasodilatation or neurogenic inflammation ${ }^{32,460}$.

In rats, skin blood flow may be influenced by excitation of both sympathetic ${ }^{556}$ and anti dromically acting sensory nerve fibres ${ }^{174,339}$. Consequently, skin blood flow may be used as a measure of efferent (vasomotor) function of both types of nerve fibres. These considerations prompted us to assess skin blood flow in the hind paw of the rat before, at an early stage (day 4) and at a later stage (day 21) after loose ligation of the ipsilateral sciatic nerve. At the two time-points after ligation, skin blood flow was assessed before and after (chemical) blockade of sensory and non-sensory nerve fibres in the loosely ligated sciatic nerve. Thus, the involvement of both types of nerve fibres in skin blood flow abnormalities caused by this type of nerve injury could be explored.

MATERIALS AND METHODS (for detailed information see chapter 4)

\section{Materials}

All 32 male Lewis rats used were subjected to loose ligation of the right sciatic nerve.

\section{Experimental protocol}

In two groups of 8 rats each, LDF was employed to measure skin blood flow (SBF) before ligation as well as at day 4 (group I, experiment Ia) or day 21 (group II, experiment Ila) after ligation, respectively. At the latter two time-points, SBF measurements were performed before and after exposure of the loosely ligated sciatic nerve. Following exposure of this nerve, the capability of sensory fibres in this nerve to mediate a vasodilator response was abolished. To this end, capsaicin was applied perineurally at midthigh level. SBF was again assessed at 10 and 60 minutes following the capsaicin application. 
Following this one hour observation period (during which the influence of capsaicin application on SBF was assessed), we applied a $2 \%$ lidocaine solution to the capsaicinpretreated sciatic nerve to block impulse propagation of non-sensory nerve fibres. Since so far no evidence has been presented for the involvement of other than sensory and sympathetic nerve fibres in neurogenic skin vasomotor control, the effect of lidocaine application to capsaicin pretreated nerwes on skin blood flow abnormalities most likely can be attributed to blockade of impulse propagation of sympathetic nerve fibres. SBF was then again determined 10 minutes later (group I, experiment Ib; group II, experiment IIb).

In two other groups of 8 rats each, LDF was employed to obtain information on systemic effects of lidocaine application on SBF abnormalities caused by ipsilateral nerve injury. To this end, SBF was assessed in the hind paw before ipsilaterall loose sciatic nerve ligation as well as at day 4 (group III) or day 21 (group IV) after ligation, respectively. At either time-point after ligation, a surgical wound was created in the left fore paw of the rats through blunt dissection of the triceps brachii muscle. The size of the surgical wound in the fore paw was comparable to the size of the surgical wound in the hind paw used for the conduction blockades of the loosely ligated sciatic nerve. Lidocaine was then applied (over a 10 minutes period) in such a way that there was continuously (over a 10 minutes period) a layer of this solution in the surgical wound. The total amount of lidocaine used was $1 \mathrm{ml}$ for each experiment. SBF measurements in the hind paw were repeated 10 minutes after starting the lidocaine application.

\section{RESULTS}

\section{Skin blood flow (SBF) in ligated rats at day 4}

Fig. 12.1 shows that in group I, when compared with preligation values (normalised value $=$ 1 , interquartile range absolute SBF values $37-66$ perfusion units), SBF was increased ( $p<$ 0.05 ) at day 4 (1.94). Exposure of the nerve did not change SBF (1.68). Subsequent application of capsaicin to the loosely ligated sciatic nerve (experiment la) resulted in a temporary increase in ipsilateral SBF immediately upon application (data not shown). This increase, however, reached a maximum within several minutes after the removal of the piece of cotton containing the capsaicin solution. Thereafter, the initial increase was followed by a decrease in ipsilateral SBF. As a result, when compared with pre-exposure values, SBF was reduced $(\mathrm{P}<0.05)$ following capsaicin application, being 0.84 at 10 minutes. As a result of this reduction, SBF at this time-point did not differ from preligation values anymore. SBF did not decrease any further between $10(0.84)$ to $60(0.87)$ minutes following capsaicin application. Fig. 12.1 also demonstrates that subsequent lidocaine application to capsaicin-pretreated nerves (experiment Ib) further reduced SBF. As a result of this reduction, SBF was decreased $(p<0.05)$ at 10 minutes after the start of the lidocaine application $(0.50)$, when compared with SBF values obtained at 60 minutes following capsaicin application and tended to be decreased when compared with preligation values. 


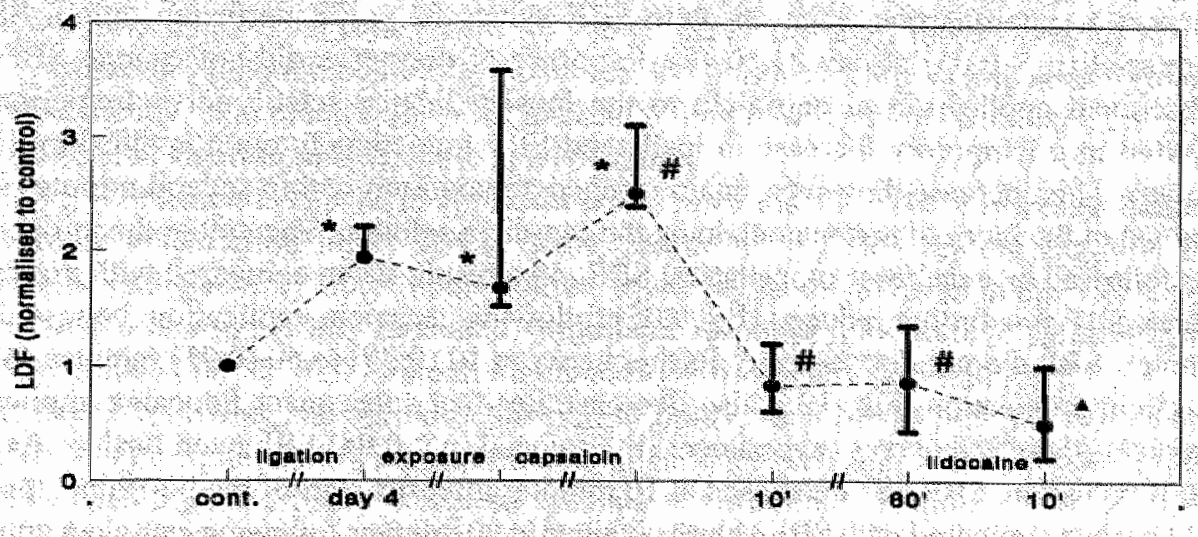

Fig. 12.1: SBF values as measured in rat skin before partial nerve injury and at day 4 (group ) At this timepoint before and after exposure of the sciatic nerve, at 10 and 60 minutes after blockade of sensory nerve fibres by capsaicin, and at 10 minutes after subsequent blockade of conduction of nonsensony nerve fibres by lidocaine All data are normalised to preligation values $(-1)$ and presented as medians (dots) and interquartile ranges (error bars) * $\mathrm{p}<0.05$ as compared to SBF values before ligation, \# $\mathrm{p}<0.05$ as compared to SBF values at day 4 (before exposure),, $\mathrm{p}<0.05$, as compared to SBF values at day 4 (after capsalicin application).

Table 12.1 shows that in group IV (evalution possible systemic effects of lidocaine 4 days after ligation), when compared with preligation values (normalised value $=1$, interquartile range absolute SBF values $23-67$ perfusion units), SBF was increased $(p<0.05)$ at day $4(2.16)$. Creation of a surgical wound in a fore paw did not influence SBF values in the hind paw (2.51). As compared to the values obtained before creation of the surgical wound, subsequent application of lidocaine into this wound did not change SBF in the hind paw, being 2.11 at 10 minutes after the lidocaine application.

$\begin{array}{llll}\text { pre- } & \text { postligation, } & \text { postligation, } & \text { postligation, } 10 \text { minutes } \\ \text { ligation } & \text { pre-exposure } & \text { postexposure } & \text { after lidocaine application }\end{array}$

\begin{tabular}{lllll} 
group III & 1 & $2.16^{\circ}$ & $2.51^{\circ}$ & $2.11^{\circ}$ \\
(day 4) & $(23-67)$ & $(1.57-3.56)$ & $(1.78-4.67)$ & $(1.56-3.29)$ \\
& & & & \\
group IV & 1 & $0.47^{\circ}$ & $0.56^{\circ}$ & $0.40^{\circ}$ \\
(day 21) & $(39-113)$ & $(0.36-0.56)$ & $(0.49-0.61)$ & $(0.27-0.72)$ \\
\hline
\end{tabular}

Table 12.1: laser Doppler flow (LDF) values as measured in rat hind paw skin at days 4 (group III) and 21 (group IV), respectively; before and after creation of a surgical wound in a fore paw and at 10 minutes after application of lidocaine into this wound. All data are normalised to preligation values $(=1)$, and presented as medians and interquartile ranges. ": $p<0.05$; as compared to preligation SBF values. 


\section{Skin blood flow in ligated rats at day 21}

Fig. 12.2 demonstrates that in group II, when compared with preligation values (normalised value $=1$, interquartile range absolute SBF values $54-127$ perfusion units), SBF was decreased $(p<0.05)$ at day $21(0.45)$. Exposure of the nerve did not change SBF $(0.43)$. Subsequent application of capsaicin to the loosely ligated sciatic nerve (experiment $\Pi_{a}$ ) resulted in a temporary increase in ipsilateral SBF immediately upon application (data not shown). This increase, however, again reached a maximum within several minutes after the removal of the piece of cotton containing the capsaicin solution. Thereafter, the initial increase was followed by a decrease in ipsilateral SBF. As a result, when compared with pre-exposure values, SBF was further reduced $(\mathrm{P}<0.05)$ following capsaicin application, being 0.29 at 10 minutes. SBF did not decrease any further between $10(0.29)$ to $60(0.21)$ minutes following capsaicin application. Fig. 12.2 also demonstrates that subsequent lidocaine application to capsaicin-pretreated nerves (experiment IIb) reduced $(p<0.05)$ SBF even further. As a result of this reduction, SBF was decreased at 10 minutes after the start of the lidocaine application $(0.11)$, when compared with SBF values obtained at 60 minutes following capsaicin application $(\mathrm{p}<0.05)$ as well as preligation values $(\mathrm{p}<0.05)$.

Table 12.1 also demonstrates that in group IV (evaluation possible systemic effects of lidocaine 21 days after ligation), when compared with preligation values (normalised value = 1 , interquartille range absolute SBF values $39-113$ perfusion units), SBF was decreased ( $p<$ 0.05 ) at day $21(0.47)$. Creation of a surgical wound in the fore paw did not influence SBF values in the hind paw $(0.56)$. As compared to the values obtained before creation of the surgical wound, subsequent application of lidocaine into this wound did not change SBF in the hind paw, being 0.40 at 10 minutes after the lidocaine application.

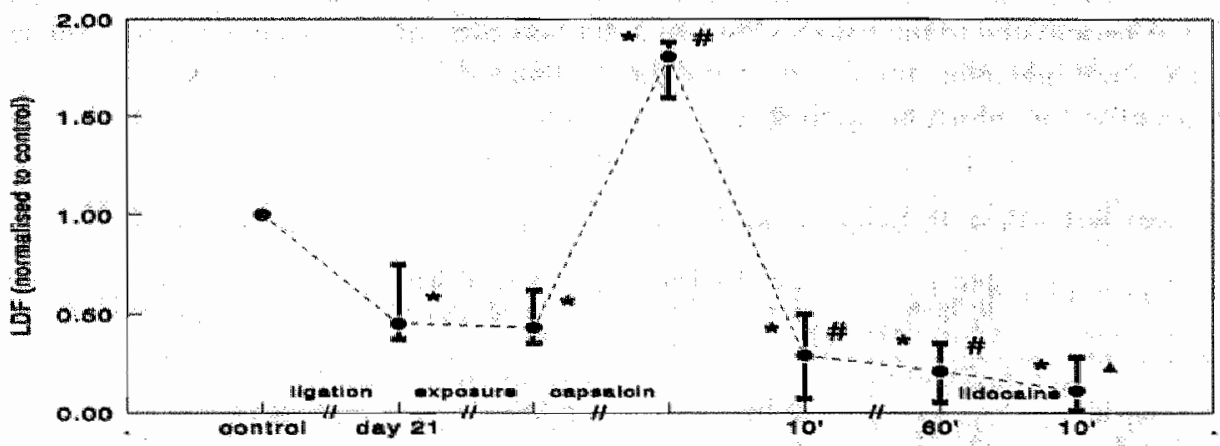

Fig. 12.2. SBF values as measured in rat skin before partial nerve injury and at day 21 (group 11), At this timepoint before and after exposure of the sciatic nerve, at 10 and 60 minutes after blockade of sensory nerve fibres by capsaicin, and at 10 minutes after subsequent blockade of conduction of nonsensory nerve fibres by Hidocaine. All data ane normalised to preligation values ( $=1$ ), and presented as medians (dots) and interquartile ranges (error bars). On the $Y$-axis SBF. On the X-axis the different time points. $: p<0.05$, as compared to SBF values before ligation; : $p<0.05$, as compared to SBF values at day 4 (before exposure) $+p<0.05$, as compared to SBF values at day 4 (after capsaicin application). 


\section{DISCUSSION}

This study shows that partial injury of a rat sciatic nerve (througli loose ligation) affects efferent (vasomotor) function of both sympathetic and antidromically acting sensory nerve fibres. Consequently, skin blood flow in the ipsilateral hind paw develops from an increase at an early stage after ligation to a decrease at a later stage.

At day 4, perineural capsaicin application overruled the wasodilator response induced by the chronic constriction injury $(\mathrm{CCl})$ of the sciatic nerve. This finding suggests that antidromically acting sensory fibres in the injured sciatic nerve are involved in this vasodilator response. Such a neurogenically mediated vasodilator response may involve antidromically acting unmyelinated $(C)$ as well as small myelinated $(A-\delta)$ sensory nerve fibres ${ }^{255}$.

At day 4 following the chronic constriction injury, skin blood flow did not change between 10 to 60 minutes following capsaicin application which suggests that at both timepoints the capability of sensory fibres to mediate a vasodilator response was abolished completely. Hence, the effect of subsequent lidocaine application on skin blood flow most likely results from blockade of impulse propagation of non-sensory nerve fibres. Our finding that lidocaine application further reduced skin blood flow suggests that at this early stage nonsensory nerve fibres are also involved in the vasodilator response induced by partial nerve injury. Since so far no evidence has been presented for the involvement of other than sensory and sympathetic nerve fibres in neurogenic skin vasomotor control, the effect of blockade of impulse propagation of non-sensory nerve fibres most likely can be attributed to blockade of sympathetic nerve fibres. A sympathetically mediated vasodilator response may involve activation of (vasodilator) B-adrenoceptors. However, so far no direct evidence for the existence of $B$-adrenoceptors in rat skin is available, which suggests that another (sympathetically mediated) vasodilator mechanism must be involved. Such a mechanism may involve release of (vasodilator) neuropeptides at the endings of sympathetic nerve fibres. Although no direct evidence for the existence of such a mechanism is available, the observation that sympathetic ganglion cells of the cat contain mRNA coding for part of the mature substance $P$ may argue in favour of this hypothesis ${ }^{329}$.

At day 21 following the chronic constriction injury, skin blood flow was decreased when compared with preligation values. Blockade of impulse propagation of non-sensory nerve fibres (lidocaine application to capsaicin pretreated nerves) even further reduced skin blood flow, which argues against the hypothesis that an increased level of efferent sympathetic (vasoconstrictor) nerve impulses is responsible for the decrease in skin blood flow (or skin temperature) at a later stage after partial nerve injury ${ }^{39}$. This finding is in line with the findings of Wakisaka et al ${ }^{535}$ who demonstrated in CCI rats that surgical sympathectomy of the affected limb does not have a significant effect on the lowered skin temperature as observed at two weeks following partial nerve injury. Thus, the decrease in skin blood flow at day 21 is likely to be humorally mediated. A humoral component may act through supersensitivity of skin microvessels to catecholamines. It has been demonstrated in vascular structures that such a supersensitivity-phenomenon may result from decreased contact between catecholamines and smooth muscle cells ${ }^{36,42}$. The presence of the latter phenomenon in the chronic constriction injury model is supported by the observation of Wakisaka and colleagues ${ }^{533}$, who demonstrated that the density of sympathetic nerve fibres in foot pad arteries from the ligated side is reduced. 
This reduced density may result from interruption of sympathetic efferents at the site of loose ligation. The hypothesis on interrupted sympathetic efferents is supported by the observation of axonal degeneration of small unmyelinated (most likely also sympathetic) nerve fibres in the loosely ligated sciatic nerve ${ }^{69,376}$. In these studies, axonal degeneration of small unmyelinated nerve fibres varied from $20 \%{ }^{376}$ to as much as $84 \%$.

At day 21 , perineural application of capsaicin further reduced skin blood flow, which indicates that in the chronic constriction injury model antidromically acting sensory nerve fibres are involved in a vasodilator response not only at an early, but also at a later stage after ligation. Skin blood flow did not change between 10 to 60 minutes following capsaicin application which again indicates that at both time-points the capability of sensory fibres to mediate a vasodilator response was abolished completely. Besides, our finding that subsequent bleckade of non-sensory nerve fibres even further reduced skin blood flow suggests that these fibres are also involved in a vasodilator response at a later stage. As alluded to before, the latter mechanism most likely involves sympathetic nerve fibres. The vasodilator response at this later stage, however, is overruled by a non-neurogenic vasoconstrictor mechanism.

It has been demonstrated previously that electrical excitation of peripheral nerves provokes ipsilateral vasodilatation ${ }^{174}$. This study, however, is the first to suggest that partial injury of a peripheral nerve through loose ligation provokes an ipsilateral vasodilator response.

We have demonstrated that skin blood flow in the affected extremity of patients with nerve injury develops from an increase at an early stage to a decrease at a later stage s98,299. $^{2}$. Besides, other studies have presented indirect evidence for autonomic denervation ${ }^{138,298}$ and supersensitivity to catecholamines of vascular structures in the affected limbs of patients with partial nerve injury ${ }^{19}$. The similarity in clinical signs and symptoms, microcirculatory abnormalities, and indices of sympathetic dysfunction warrants the usefulness of the chronic constriction injury model in the evaluation of the neuropathophysiological mechanisms involved in patients with partial nerve injury (such as causalgia patients).

The findings in our study attest to the presence of a neurogenic vasodilator mechanism in the chronic constriction injury model. As alluded to before, such a neurogenic mechanism acts through release of neuropeptides from the peripheral endings of primary afferents. Neuropeptides have additionally been reported to increase the excitability of primary sensory nerve fibres ${ }^{132}$ and to increase vascular permeability ${ }^{318}$. Hence, in case of partial nerve injury, the alleged release of neuropeptides may be related not only to vasodilation, but also to sensory abnormalities and edema formation, respectively. The latter concept may raise the possibility of new therapeutic approaches (such as neuropeptide-antagonists) for patients with traumatic partial nerve injury.

In conclusion, the present study indicates that, in the rat, partial sciatic nerve injury induces ipsilaterally an increase in skin blood flow at an early stage, which is followed by a decrease at a later stage. At both stages, neurogenic mechanisms, mediated by antidromically acting sensory and orthodromically acting non-sensory (most likely sympathetic) nerve fibres, are involved in an ipsilateral vasodilator response. At a later stage in this model, however, neurogenic vasodilator mechanisms are overruled by a non-neurogenic vasoconstrictor mechanism. This vasoconstrictor mechanism may consist of supersensitivity of skin microvessels to catecholamines consequent to reduced neurogenic disposition of catecholamines. 


\section{Chapter 13}

IN VITRO INVESTIGATION OF SYMPATHETIC DYSFUNCTION IN REFLEX SYMPATHETIC DYSTROPHY PATIENTS AND IN RATS WITH PARTIAL NERVE INJURY:

\section{DENERVATION-INDUCED SUPERSENSITIVITY TO CATECHOLAMINES}

HAJM Kurvers, FRM Stassen, RS Reneman, GJ Tangelder, DW Slaaf, FAJM van den Wildenberg, PJEHM Kitslaar, MARC Daemen, MJHM Jacobs, and JGR De Mey

Departments of Surgery of the University Hospital Maastricht and of the Academic Medical Centre Amsterdam, The Netherlands.

Departments of Surgery, Pharmacology "Physiology, and Biophysics of the Cardiovascular Research Institute Maastricht (CARIM), The Netherlands. 


\section{SUMMARY}

Loose ligation of a sciatic nerve in rats (chronic constriction injury $(\mathrm{CCl})$ ) induces signs and symptoms like those observed in clinical conditions of neuropathic origin, such as reflex sympathetic dystrophy (RSD). Sympathetic dysfunction in the CCl model as well as in RSD has been suggested to involve denervation-induced supersensitivity to catecholamines. We, therefore, studied structural and functional aspects of sympathetic innervation in subcutaneous arteries, that had been isolated from the hind paw of rats with 3 week old $\mathrm{CCl}$ and from amputated extremities of stage III (chronic cold sensation) RSD patients. The density of sympathetic nerve fibres was assessed histologically. In a myograph, we recorded contractile responses to stimulation of adrenergic nerves and exogenously administered adrenergic agonists. In CCI rats, as compared to the contralateral side, subcutaneous arteries from the ligated side; 1) were partially denervated, 2) showed increased sensitivity to $\alpha_{1}$-adrenoceptor stimulation, and 3) demonstrated reduced presynaptic uptake of catecholamines. No alterations in structural dimensions of the vessel wall were observed. Similar alterations were observed in arteries of stage III RSD patients. This study demonstrates that (arterial) sympathetic dysfunction in an experimental model of neuropathic pain as well as in RSD patients involves denervation-induced supersensitivity to catecholamines. This supersensitivity phenomenon results from impaired presynaptic reuptake of and postjunctional supersensitivity to catecholamines.

\section{INTRODUCTION}

Loose ligation of a sciatic nerve in rats (chronic constriction injury (CCI)) has been shown to result in signs and symptoms comparable to those seen in clinical conditions of neuropathic origin, such as causalgia and reflex sympathetic dystrophy (RSD) ${ }^{39,40,392,507}$. In addition to sensory and motor disturbances, this type of nerve injury induces autonomic dysfunction, characterised among others by sympathetically maintained sensory abnormalities ${ }^{281,382,474}$, trophic changes ${ }^{40}$, as well as abnormalities in skin temperature ${ }^{39,40,533}$ and skin blood flow ${ }^{303}$.

So far, the exact nature of autonomic dysfunction in RSD patients and experimental animal models of neuropathic pain remains enigmatic. Sympathetic dysfunction has been purported to consist of an increase in efferent sympathetic nerve impulses, because surgical or chemical sympathectomy may relieve sensory abnormalities and improve skin blood flow in RSD patients $\mathrm{s}^{4,231,545}$ as well as in experimental animal models of neuropathic pain ${ }^{39,281,382}$. However, some recent studies have presented evidence of autonomic denervation in the affected limb, both in the $\mathrm{CCI}$ model ${ }^{533}$ and in RSD patients ${ }^{138,298}$. Autonomic denervation of vascular structures increases their sensitivity to catecholamines ${ }^{36,42,233,499}$. Hence, sympathetic dysfunction in RSD patients as well as in the CCI model may involve denervation-induced supersensitivity to catecholamines.

Structura $\left.\right|^{388}$ and functional ${ }^{402}$ aspects of sympathetic innervation as well as sensitivity to adrenergic agonists ${ }^{386}$ can be investigated in subcutaneous arteries. This prompted us to study these aspects in arteries that had been isolated from the hind paw of rats with 3 week old $\mathrm{CCI}$ and from amputated extremities of stage III (chronic cold sensation) RSD patients. The 
structural density of sympathetic nerve fibres was assessed histologically. In a myograph, we recorded contractile responses to stimulation of adrenergic nerves (fuinctional density) and exogenously administered agonists (adrenoceptor function).

\section{MATERIALS AND METHODS (for detailed information see chapter 4)}

\section{Experimental animal model}

The experiments were performed on subcutaneous arteries isolated from 17 male Lewis rats. Twelve rats were subjected to loose ligation of the right sciatic nerve. On the contralateral (left) side, the sciatic nerve was exposed, but not ligated. In the remaining 5 rats (control group), we only performed a sham-procedure during which the right sciatic nerve was exposed, but not ligated. Three weeks later, the animals were killed by a sharp blow on the neck and exsanguinated. Immediately after sacrifying the animals, we isolated from the plantar surface the most laterally located subcutaneous artery. In the ligated group this was done in both hind paws (ligated side: LIG; non-ligated side: NONLIG) and in the control group (CON) in the left hind paw. In arteries isolated from both hind paws of the ligated rats (i.e. LIG AND NONLIG), four sets of experiments were performed. In the first series, (intramural) noradrenaline containing nerves were visualised in a small segment of the isolated artery with the use of glyoxylic acid ${ }^{330}$. An independent investigator, who was blinded as to the side from which the vessel segment had been obtained, scored the density of the intramural sympathetic nerves as follows: increased, no differences, or decreased when compared with the vessel segment obtained from the contralateral side. In order to examine mechanical properties, the remaining segment of the isolated arteries was placed horizontally in a myograph between an isometric force transducer and a displacement device for recording of isometric force development ${ }^{107,375}$. Before experimentation, all arteries were distended to their individual optimal lumen diameter, i.e. that yielding the maximal force development. In the second series, contractile responses to electrical field stimulation (EFS) were evaluated. In our experimental set-up, vascular responses to EFS exclusively resulted from stimulation of intramural adrenergic nerves. In the third series, contractile responses to cumulative doses of various adrenergic agonists were assessed. Concentration-response curves were obtained by increasing the concentrations in half-log increments. Sensitivities to the agonists were calculated by interpolation on a logistic curve fit of the individual concentration response curves. Concentration response curves ( 10 $\mu \mathrm{M}-30 \mu \mathrm{M})$ were constructed for the non-selective adrenoceptor agonist noradrenaline, the $\alpha_{1}$-selective agonist phenylephrine and the $\alpha_{2}$-agonist B-HT 933 (azepexole). The ratio of the maximal response to EFS and the maximal response to noradrenaline was calculated as a functional measure of adrenergic innervation. $B$-adrenergic responses were investigated by constructing concentration-response curves for isoprenaline after precontracting the arteries with $35 \mathrm{mM} \mathrm{K}^{+}$. Concentration-response curves for isoprenaline were constructed and maximal relaxing responses were expressed as percentage of the precontraction. Initially, vasoconstrictor (noradrenaline, phenylephrine, B-HT 933) and vasodilator (isoprenaline) responses to these adrenoceptor agonists were assessed without blockade of presynaptic reuptake of catecholamines. In order to investigate the efficacy of the latter mechanism, we subsequently studied the influence of $3 \mu \mathrm{M}$ cocaine (which blocks presynaptic reuptake of catecholamines) 
on the sensitivity of arteries for noradrenaline $(10 \mu \mathrm{M}-30 \mu \mathrm{M})$. In the fourth series, we studied the structural dimensions of vessel segments in which contractile responses to EFS and various pharmacological agents had been assessed. To this end, these vessel segments were fixed at their optimal diameter. Preparations were paraffin embedded and cross sectioned using standard histological techniques. Cross sections $(4 \mu \mathrm{m})$ were stained for elastin. The internal and external medial circumference were measured, which were respectively demarcated by the internal and external elastic lamina. From these, assuming circular vessel conformation, the lumen area and the total area could be derived. Medial cross sectional area (CSA) was obtained by subtracting lumen area from the total area. Internal lumen radius (r) was calculated from the internal circumference, again assuming a circular vessel conformation. From these parameters, average media thickness $\left(M_{1}\right)$ was calculated. Finally, the wall to lumen ratio (W/L) was calculated.

\section{Statistics}

The contractile forces which were elicited at each frequency of electrical stimulation and each concentration of adrenergic agonist, are expressed as the force divided by twice the vessel segment length (in $\mathrm{mN} / \mathrm{mm}$ ). In the ligated group, all experimental data from the ligated side (LIG) were compared with those from the contralateral side (NONLIG). Besides, we compared contractile responses (to adrenergic agonists and EFS) of the left hind paw of the ligated group (NONLIG) with those from the left hind paw of the control group (CON). This was done in order to exclude the possibility that loose sciatic nerve ligation alters vascular reactivity in the contralateral hind paw.

\section{Reflex sympathetic dystrophy (RSD) patients}

In order to investigate whether sympathetic dysfunction in RSD patients is qualitatively similar to sympathetic dysfunction in rats subjected to loose sciatic nerve ligation, we additionally investigated sympathetic innervation and sensitivity to catecholamines of arteries isolated from subcutaneous fat of amputated extremities of 2 RSD patients. All experimental procedures were performed according to procedures described above. Experimental data from the neuropathic side were compared with those from arteries isolated from subcutaneous fat of the contralateral extremity. Both patients had given informed consent in advance to the operative procedure and met standards of RSD described in detail in chapter 4 . The areas of the amputated limbs from which the subcutaneous arteries were obtained were affected by RSD, with respect to both sensory abnormalities and perception of abnormal skin temperature.

\section{Patient history}

Patient A, a 42 year old female, suffered from RSD for about 12 years. The syndrome had been initiated by a bimalleolar fracture of the right arikle with severe soft tissue damage. The fracture had been treated operatively by means of external fixation. Patient history and physical examination revealed, among others, the following features of RSD: 1) autonomic (oedema, abnormal nail growth, cold/pale skin), 2) trophic (cutaneous atrophy, abnormal consistency of nails), 3) motor (reduced strength, muscle wasting, involuntary movements), and 4) sensory (ongoing burning pain, hyperalgesia, allodynia). Lower leg amputation was performed because of severe deformation of the affected extremity which limited mobility. Patient $B$, a 53 year old 
female, suffered from RSD for $4 \frac{1}{2}$ years. The syndrome had been initiated by a conminuted intra-articular fracture of the left wrist which had been treated conservatively by means of a cast. Patient history and physical examination revealed; among others, the following features of RSD: 1) autonomic (edema, increased edema on dependency, cold/pale skin, abnormal hair growth), 2) trophic (cutaneous atrophy, abnormal consistency of nails), 3) motor (reduced strength, muscle wasting, tremor), and 4) sensory (ongoing burning pain, hyperalgesia). Amputation (at lower arm level) was performed because of severe deformation of the affected extremity.

\section{RESULTS}

\section{Experimental animal model}

In the ligated group, the density of (intramurall) sympathetic nerve fibres on the ligated side was reduced $(\mathrm{p}<0.05)$ in 10 out of 12 rats when compared with the non-ligated side (for typical example see fig 13.1). A striking phenomenon was that a considerable interindividual difference existed in the extent of autonomic denervation.
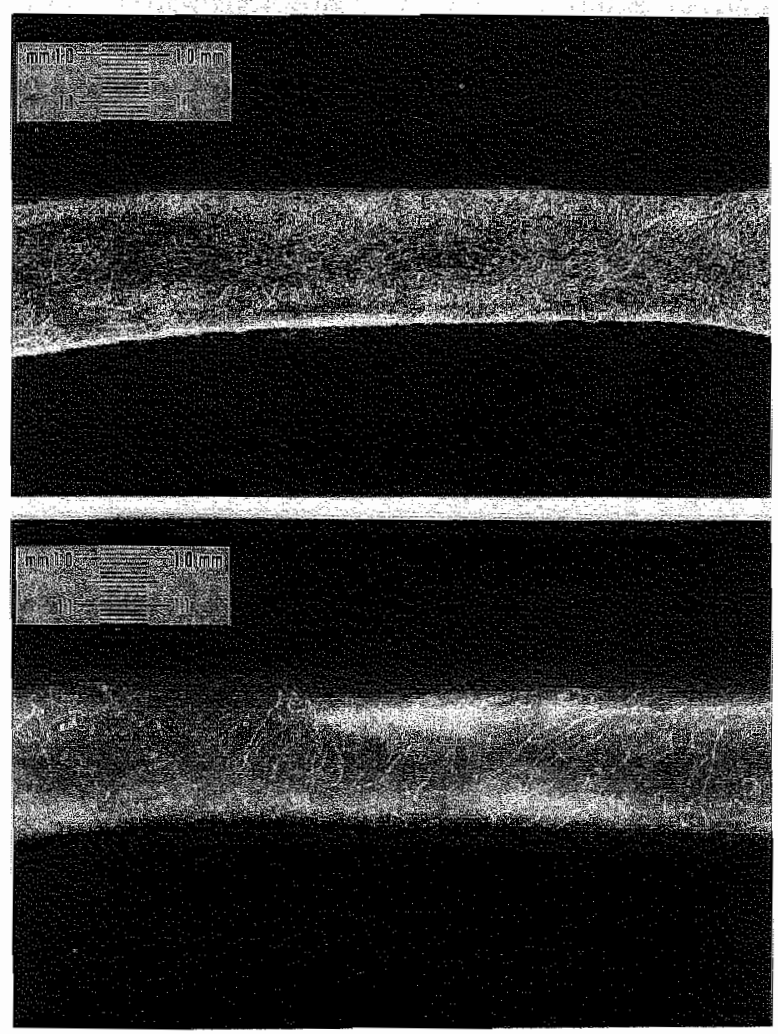

Fig. 13.1: typical example of rat arteries from left (non-ligated, top) and right (ligated, bottom) hind paw. Sympathetic nerve fibres were visualised with glyoxylic acid-induced fluorescence. 
The optimal lumen diameter at which maximal contractile responses to 5-HT were obtained did not differ, either between the ligated (LIG) and non-ligated (NONLIG) side in the ligated group $(271 \pm 10 \mathrm{vs} 274 \pm 10 \mu \mathrm{m})$, or between the NONLIG and the control group (CON) (274 \pm 10 vs $275 \pm 30 \mu \mathrm{m})$. Maximal contractile responses to $5 \mathrm{HT}(10 \mu \mathrm{M})$ also did not differ between these groups (LIG: $5.40 \pm .30$; NONLIG $5.29 \pm .28$; CON $4.58 \pm .46 \mathrm{mN} / \mathrm{mm}$ ).

The stimulation parameters used during EFS (range $0.25-32 \mathrm{~Hz}$ ) elicited distinct contractions during the course of each experiment (fig. 13.2). In the ligated group, these responsess were for the whole frequency range decreased on the ligated side when compared with the non-ligated side $(3.71 \pm .69$ vs $5.68 \pm .35 \mathrm{mN} / \mathrm{mm}$ at $32 \mathrm{~Hz}, \mathrm{p}<0.05)$. No differences were observed in maximal contractile responses to EFS between NONLIG and CON ( $5.68 \pm$ .35 vs $4.84 \pm .53 \mathrm{mN} / \mathrm{mm}$ ). In the ligated group, the ratio of maximal responses to EFS divided by the maximal responses to noradrenaline was also reduced on the ligated side, when compared with the non-ligated side $(.94 \pm .03$ vs $.61 \pm .20, \mathrm{p}<0.05)$. No differences in this ratio were observed between NONLIG and CON (.84 \pm .04 vs $.94 \pm .03)$.

All arteries demonstrated concentration-dependent contractions in response to the $\alpha_{1}$ adrenergic agonist phenylephrine. Fig. 13.3 shows that in the ligated group, the sensitivity for phenylephrine was increased on the ligated side, when compared with the non-ligated side $\left(\mathrm{pD}_{2}\right.$ $6.25 \pm .08$ vs $5.99 \pm .07, \mathrm{p}<0.05$ ), whereas no differences were observed in the maximal responses induced by phenylephrine ( $5.68 \pm .37$ vs $5.47 \pm .23 \mathrm{mN} / \mathrm{mm})$. No differences were observed between NONLIG and CON, either in sensitivity for ( $\mathrm{pD}_{2} 5.99 \pm .07 \mathrm{vs} 5.82 \pm .09$ ) or in maximal responses elicited by phenylephrine $(5.47 \pm .23$ vs $5.03 \pm .65 \mathrm{mN} / \mathrm{mm})$. The $\alpha_{2^{-}}$ selective agonist B-HT 933 did not evoke contractile responses in arteries of either the ligated or the control group.

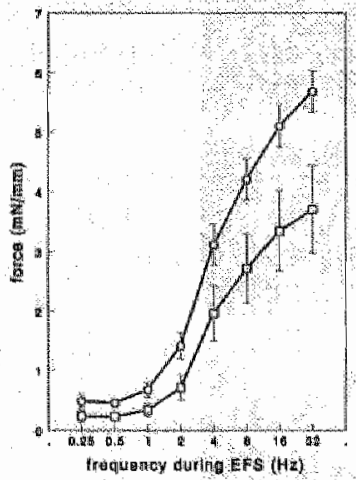

Fig. 13.2: frequency force relations during electrical field stimulation (EFS) in rat atteries from the ligated side (open square) and non-ligated side (open circle). Increases in wall tension are shown as mean values \pm $\operatorname{SEM}(n=12)$.

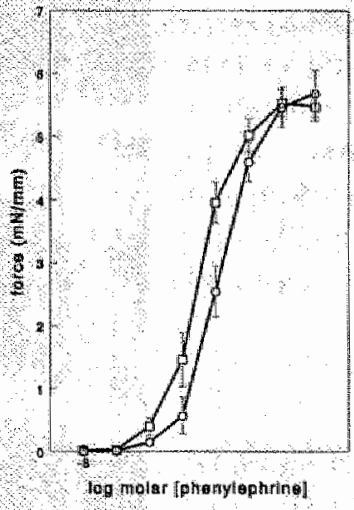

Fig. 13.3. concentration-force relations for phenylephrine in rat arteries from the ligated side (open square) and non-ligated side: (open circle). Increases in wall tension are shown as mean values \pm SEM $(n=12)$. 
In all arteries, concentration-dependent contractions were evoked by noradrenaline. Since no contractile responses to the $\alpha_{2}$-selective agonist $\mathrm{B}-\mathrm{HT} 933$ were observed, it is likely that these contractile responses reflect activation of $\alpha_{1}$-adrenoceptors. As is shown in fig. 13.4, in the ligated group the sensitivity for noradrenaline was increased on the ligated side, when compared with the non-ligated side $\left(\mathrm{pD}_{2} 6.57 \pm .12\right.$ vs $\left.6.10 \pm .07, \mathrm{p}<0.01\right)$, whereas no differences were observed in the maximal forces induced by noradrenaline $(5.97 \pm .30$ vs 5.87 $\pm .34 \mathrm{mN} / \mathrm{mm}$ ). No differences were observed between NONLIG and CONTROL, either in sensitivity for $\left(\mathrm{pD}_{2} 6.10 \pm .07\right.$ vs $\left.6.08 \pm .15\right)$ or maximal response induced by noradrenaline (5.87 \pm .34 vs $5.69 \pm .49 \mathrm{mN} / \mathrm{mm}$ ). A negative correlation was observed between $\mathrm{pD}_{2}$ values for noradrenaline and maximal contractile responses to EFS in $L I G(N=12, p<0.01, R=-$ $0.75)$, but not in NONLIG $(\mathrm{N}=12, \mathrm{R}=-0.48)$ Nor in $\mathrm{CON}(\mathrm{N}=5, \mathrm{R}=-0.01)$.

As is illustrated in fig. 13.5, blockade of presynaptic uptake of catecholamines by means of cocaine ( $3 \mu \mathrm{M})$ increased in the ligated group the sensitivity to noradrenaline on the nonligated side $\left(\mathrm{pD}_{2} 6.31 \pm .15 \mathrm{vs} 6.10 \pm .07, \mathrm{p}<0.05\right)$, whereas on the ligated side the observed change was smaller and did not reach statistical significance $\left(\mathrm{pD}_{2} 6.68 \pm .15\right.$ vs $\left.6.57 \pm .12\right)$. Cocaine also increased the sensitivity for noradrenaline in the CON group $\left(\mathrm{pD}_{2} 6.08 \pm .15 \mathrm{vs}\right.$ $6.71 \pm .13, \mathrm{p}<0.05)$.

The $B$-adrenoceptor agonist isoprenaline evoked concentration-dependent relaxing responses in all arteries. In the ligated group, no differences were observed between LIG and NONLIG, either in sensitivity for isoprenaline $\left(\mathrm{pD}_{2} 7.18 \pm .09 \mathrm{vs} 7.06 \pm .16\right)$ or in the maximal relaxation ( $19 \% \pm 4$ vs $21 \% \pm 7)$ induced by this agonist.

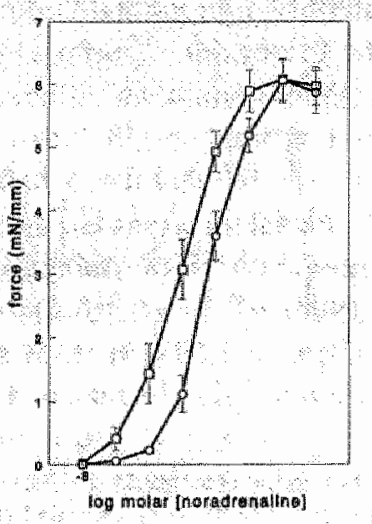

Fig. 13,4: concentration-force relations for noradrenaline in rat arteries from the ligated side (open square) and nonligated side (open circle). Increases in wall tension are shown as mean values \pm $\operatorname{SEM}(n=12)$.

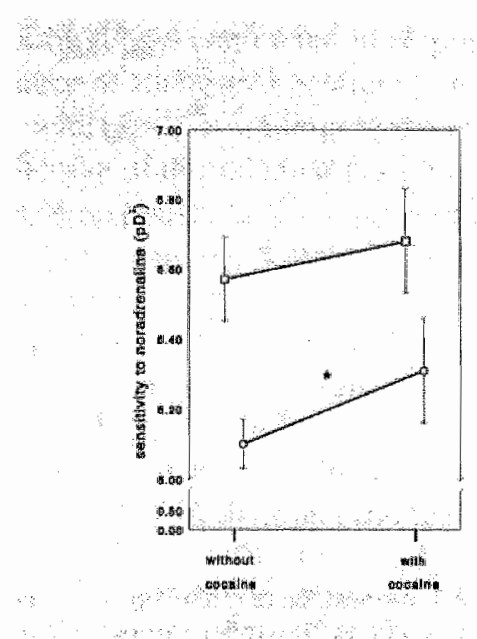

Fig. 13,5. sensitivities ( $\mathrm{pD}$, values) for noradrenaline in rat arteries from the ligated side (open square) and nonligated side (open circle), in absence and presence of $3 \mu \mathrm{M}$ cocaine. Asterisk represents significant $(P<0,05)$ increase in $\mathrm{pD}_{2}$ consequent to exposure to cocaine. 
In the ligated group, no differences were observed between the ligated and non-ligated side as regards thedia cross sectional area $\left(17,170 \pm 1,110 \mathrm{vs} 17,900 \pm 920 \mathrm{~mm}^{2}\right)$, average media thickness $(17.0 \pm 1.1 \mathrm{vs} 18.0 \pm 0.9 \mu \mathrm{m})$, or wall to lumen ratio $(0.126 \pm 0.008 \mathrm{vs} 0.134 \pm$ $0.009)$ at optimal humen diameter $(274 \pm 10 \mathrm{ws} 272 \pm 10 \mu \mathrm{m})$.

\section{Reflex sympathetic dystrophy (RSD) patients}

In the subcutaneous resistance arteries isolated from the affected extremities of two patients that suffered from RSD, the findings (fig. 13.6) were qualitatively similar to those in rats subjected to loose ligation of the sciatic nerve. In case $A$, the subcutaneous resistance artery from the affected side was 5 to 10 times more sensitive to the contractile action of exogenous noradrenaline than the resistance artery at the control side. Unlike the control artery, the artery from the affected side failed to contract in response to electrical field stimulation. In case $\mathbb{B}$, the vessel from the affected extremity was 3 times more sensitive to exogenous noradrenaline than the control artery. Also in this case the affected artery hardly contracted in response to electrical nerve stimulation while in the control vessel strong frequency-dependent responses were obtained.

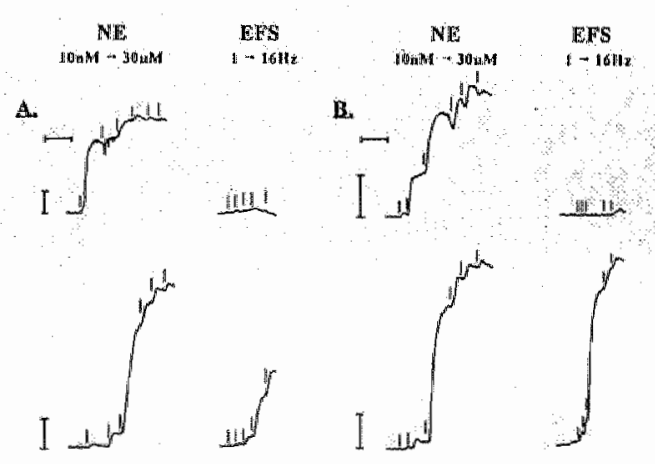

Fig. 13.6. redrawings of recordings of active wall tension versus time obtained in resistance arteries of two patients suffering from reflex sympathetic dystrophy ( $A$ and $B$ as detalled in the text). Top vessels from the affected extremity Bottom vessels from an apparently healthy extremity. NE, noradrenaline (cumulative. increases in concentrations): EFS, electrical field stimulation with stepwise increasing frequency Between stimulations, 20 - 30 minutes equilibration in drug-free solution was allowed, Calibration bars harizontal, 5 minutes; vertical, $1 \mathrm{mN} / \mathrm{mm}$. 


\section{DISCUSSION}

The findings of the present study indicate that sympathetic dysfunction in subcutaneous arteries obtained from the hind paw of rats subjected to a chronic constriction injury (CCI) of the sciatic nerve involves denervation-induced supersensitivity to catecholamines. This supersensitivity phenomenon consists of impaired presynaptic reuptake of and postjunctional supersensitivity to catecholamines. Similar changes were observed in amputated extremities of two reflex sympathetic dystrophy (RSD) patients.

In arteries obtained from the ligated side of CCI rats, we observed reduced adrenergic nerve fibre density as well as impaired contractile responses to electrical field stimulation. These findings are indicative of, respectively, structural and functional sympathetic denervation. Besides, presynaptic reuptake of noradrenaline was impaired on the ligated side, which is also indicative of sympathetic denervation. This (partial) sympathetic denervation may be related to degeneration of small unmyelinated (most likely also sympathetic) axons in the loosely ligated sciatic nerve, which has been reported to vary from $20 \%{ }^{376}$ to as much as 84 $\%$. Wakisaka and colleagues ${ }^{533}$ have demonstrated previously in $\mathrm{CCl}$ rats a reduced number of sympathetic nerve fibres in plantar arteries and veins. These authors, however, observed an almost complete absence of sympathetic nerve fibres whereas in our study a variable reduction was found. This dissimilarity may result from differences in 1) the degree to which the nerve is constricted; 2) rat strain used ${ }^{206}$, and 3) age and size of the rats ${ }^{507}$. Another finding which is in line with the histological observation of (partial) sympathetic denervation is that in these rats the vasoconstrictor response to an autonomic provocation test was reported to be ongoingly impaired ${ }^{303}$, which also suggests of sympathetic denervation ${ }^{328}$.

Control arteries from the plantar surface of the rat hind paw contracted in response to phenylephrine but not to B-HT 933, indicating the presence of functional postjunctional $\alpha_{1}-$ adrenoceptors but not of $\alpha_{2}$-adrenoceptors. This is in line with previous findings in mesenteric resistance arteries ${ }^{386}$, the size of which is comparable to the size of the subcutaneous hind paw arteries used in our study. Besides, assessment of contractile responses to the $\alpha_{1}$-adrenoceptor agonist phenylephrine indicated that postjunctional (adaptive) sensitivity to catecholamines is increased in arteries on the ligated side when compared with the non-ligated side. Although the observed differences in sensitivity to phenylephrine appear to be rather small, one should realise that concentration-response curves for agonists in vitro can not be compared to doseeffect relationships in the intact system. E.g., it is well known that in both rats and man catecholamines constrict isolated blood vessels only from $10 \mathrm{nM}$ onwards, while they modulate vascular resistance in vivo at normal circulating levels $( \pm 1 \mathrm{mM})$. The obvious explanation for this incongruity is that in vivo many vasoconstrictor agents act together and interact synergistically. Thus vascular tone not only depends on catecholamines but on the combination of catecholamines, angiotensin II, vasopressin, etc.

Another important mechanism involved in regulation of vascular responses to catecholamines is the presynaptic reuptake mechanism. In our study, the sensitivity to noradrenaline was increased on the ligated side when compared with the non-ligated side. Exposure of the arteries to cocaine, which blocks presynaptic reuptake, significantly increased sensitivity for noradrenaline on the ligated, but not on the non-ligated side. This finding indicates that the presynaptic reuptake mechanism is impaired on the ligated side (i.e. 
prejunctional or deviation supersensitivity). In the $\mathrm{CCl}$ model, impairment of presynaptic reuptake may be related to degeneration of small unmyelinated (most likely also sympathetic) nerve fibres in the loosely ligated sciatic nerve ${ }^{69,376}$.

Sympathetic denervation of vascular structures increases their sensitivity to cate cholamine $3^{36,42,233,499}$. Hence, sympathetic denervation as observed on the ligated side of $\mathrm{CCI}$ rats is likely to be involved in the development of arterial supersensitivity to catecholamines. The latter hypothesis is supported by the observed negative correlation between maximal contractile responses during electrical field stimulation and sensitivity to noradrenaline.

The results of some previous studies in CCI rats are in line with the hypothesis that sympathetic dysfunction induced by partial nerve injury involves supersensitivity to catecholamines consequent to (partial) sympathetic denervation. Bennett and Ochoa ${ }^{39}$ demonstrated skin temperature abnormalities in $\mathrm{CCI}$ rats. These authors suggested that the abnormal cold status of the symptomatic paw of $\mathrm{CCI}$ rats may result from increased sympathetic outflow and/or denervation-induced supersensitivity to catecholamines of vascular smooth muscle. Likewise, Wakisaka et al ${ }^{533}$ demonstrated that the average temperature of the symptomatic paw of CCI rats (for the group as a whole), is abnormally hot initially and progresses to abnormally cold at a later stage. At this later stage the number of sympathetic nerve fibres in plantar arteries and veins was found to be reduced. Consequently, the authors stated that the decrease in skin temperature at a later stage does not result from hyperactivity of noradrenergic vasoconstrictor efferents but more likely from denervation-induced supersensitivity to catecholamines.

Changes in contractile responses to electrical field stimulation and adrenergic agonists may result not only from autonomic denervation and/or altered sensitivity to catecholamines, but also from changes in vascular structural dimensions. However, morphometric analysis of subcutaneous arteries did not demonstrate any differences in vascular structural dimensions between the ligated and non-ligated side. In addition, our finding that maximum contractile responses to 5-HT, phenylephrine, and noradrenaline did not differ between both sides argues against the hypothesis of qualitative changes in the contractile machinery. In contrast, Bevan and $T$ suru ${ }^{42}$ demonstrated that surgical denervation of the central ear artery in young rabbits results in both functional and structural changes. This disparity between their findings and ours may result from various differences in experimental set-up other than the differences in species and tissues used. Firstly, in our experimental rat model the extent of sympathetic denervation was almost never complete, whereas in the rabbits of Bevan and Tsuru complete denervation was achieved. Secondly, we assessed vascular changes 3 weeks after $\mathrm{CCI}$, whereas Bevan and Tsuru studied vascular changes 8 weeks after denervation. Thirdly, structural vascular effects of sympatholytic interventions depend on the developmental stage of the animals ${ }^{100}$.

The observed differences in response to electrical field stimulation and adrenergic agonists could have resulted not only from changes in contractile response to $\alpha$-adrenergic agonists, but also from altered relaxing responses to $B$-adrenergic agonists. However, our finding that in the ligated group the relaxing responses to isoprenaline did not differ between the ligated and the non-ligated side excludes the latter hypothesis.

Our finding of (partial) sympathetic denervation in arteries from the affected side of RSD patients tallies with results from Drummond et al ${ }^{138}$ who observed a decrease in venous plasma levels of noradrenaline collected in the affected limb. Besides, we demonstrated in a 
previous study that the sympathetically mediated vasoconstrictor response of skin microvessels; Which usually follows dependency of an extremity, is impaired at all stages of the RSD syndrome $^{298}$, which may also be indicative of sympathetic denervation ${ }^{219}$.

In human arteries, we also observed increased sensitivity to noradrenaline. This finding is in line with observations of Arnold and colleagues ${ }^{19}$, who demonstrated that veins in limbs affected by RSD are supersensitive to noradrenaline. Besides, this supersensitivity phenomenon may be involved in the reduction of skin bllood flow as observed at stages II and III of the reflex sympathetic dystrophy syndrome $\mathrm{e}^{299,300}$.

We observed a similar pattern of sympathetic dysfunction in CCI rats and RSD patients, in spite of the fact that the interval between the initiating event and isolation of the subcutaneous arteries differed considerably (RSD patients $>10$ years; CCI rats 3 weeks). This similarity in sympathetic dysfunction in spite of the difference in duration of disease suggests. that 1) denervation-induced supersensitivity to catecholamines develops already at an early stage after the initiating event, and/or that 2) the rapidity with which denervation-induced supersensitivity to catecholamines develops differs between RSD patients and CCI rats. The latter hypothesis is in line with the observation that changes in skin blood flow (increase at an early stage after the initiating event followed by a decrease at later stages) develop much quicker in CCI rats $^{303}$ than in RSD patients ${ }^{299}$.

Both surgical and chemical sympathectomy may relieve some of the sensory abnormalities in RSD patients ${ }^{475}$ and $\mathrm{CCI}$ rats $^{285}$, which indicates that these sensory abnormalities are sympathetically maintained. On the basis of the findings of the present study, it is tempting to speculate that the involvement of the sympathetic nervous system in the maintenance of sensory abnormalities also involves supersensitivity to catecholamines consequent to sympathetic denervation (to which most likely not only blood vessels but also sensory nerve fibres are subjected), as has previously been suggested by Perl et al ${ }^{405}$. Thus, an increase in the number and/or responsiveness of axonal adrenoceptors might develop in peripheral nerves. Alternatively, increased adrenergic sensitivity may result from generally increased excitability of axons in peripheral nerves, on which adrenoceptors are already present but become effective only in case of partial nerve injury. The latter hypothesis seems unlikely because it implies that increased adrenergic sensitivity should always develop whenever the excitability of the peripheral axon is sufficiently increased. In contrast, various observations argue in favour of the hypothesis that the development of increased adrenergic sensitivity in injured sensory nerve fibres results from an increase in the number and/or responsiveness of axonal adrenoceptors consequent to sympathetic denervation. Firstly, sympathectomy alone may induce spontaneous pain in the sympathectomised region ${ }^{332,423,457}$, whereas it is generally agreed that, under normal conditions, activity in the sympathetic nervous system has little effect on the excitability of primary afferent nociceptors ${ }^{467}$. Secondly, it has been demonstrated in the neuroma model that $\alpha$-adrenoceptor synthesis is increased ${ }^{357}$. Thirdly, it has been demonstrated in the peripheral as well as the central nervous system that interfering with neurotransmission can lead to altered sensitivity and altered receptor binding in the effector neurons ${ }^{164}$. Fourthly, Drummond et al ${ }^{139}$ demonstrated an increase in the number of $\alpha_{1}$-adrenoceptors in the hyperalgesic skin of RSD patients. Since arterioles, sweat glands, and hair follicles were excluded from analysis, it is likely that this finding is indicative of an increased number of $\alpha_{1}$ adrenoceptors in fine intra-epidermal nerve fibres. Two main hypotheses exist as to the 
localisation of upregulated adrenoceptors in peripheral nerves. Initially, it has often been purported that the upregulated adrenoceptor is localised on the primary afferent, and that stimulation of these adrenoceptors results in activation and/or sensitisation of the primary afferent ${ }^{105}$. However, the results of a recent study in rats suggest that the adrenoceptor is localised on a sympathetic postganglionic axon, the stimulation of which provokes release of prostaglandins which, in turn, mediate the activation and/or sensitisation of primary sensory afferents $^{188}$.

Sympathectomy relieves sensory abnormalities only in part of the RSD patients. Likewise, sympathectony in CCl rats reverses some aspects of pain behaviour but not others ${ }^{281}$. These findings suggest that at least part of the sensory abnormalities are sympathetically independent and, hence, not mediated by supersensitivity to catecholamines.

Our study demonstrated considerable interindividual differences in the extent of sympathetic denervation, which, moreover, was almost never complete. Hence, a chemical or surgical sympathectomy may even further reduce the amount of noradrenaline released (prejunctionally) into the synaptic cleft. Consequently, diffusion of noradrenaline from the synaptic cleft into the blood stream decreases as a consequence of which plasma levels of this neurotransmitter become lower ${ }^{190}$. Both mechanisms may account for the observation that, although in experimental animal models of neuropathic pain as well as in RSD patients efferent sympathetic nerve activity already seems to be decreased, surgical and chemical sympathectomy may relieve sensory abnormalities and increase skin blood flow ${ }^{41,231,281,382,545}$. Nevertheless, one should realise that on the long run these sympathectomies may even further increase the level of supersensitivity to catecholamines. The latter phenomenon may account for the clinical observation that in part of the RSD patients subjected to paravertebrall sympathetic ganglion blockade relief of pain is only transient ${ }^{115,475}$.

In line with the concept of supersensitivity to catecholamines one expects that blockade of adrenoceptors may have a beneficial effect on sensory and skin blood flow abnormalities in nerve-injured rats as well as in RSD patients. The latter is supported by a study of Kim and colleagues $^{281}$ demonstrating that administration of phentolamine relieves signs of allodynia in their rat model of neuropathic pain. Phentolamine ${ }^{17}$ and phenoxybenzamine ${ }^{180}$ were reported to have beneficial effects on sensory abnormalities in humans with neuropathic pain as well. An advantage of blockade of adrenoceptors over sympathectomy could be that the long term results of the latter may be negatively influenced by a further increase in (super-) sensitivity to catecholamines. In cases where sympathetic denervation is already (sub-) complete as a result of the initiating event (nerve trauma), one may expect few beneficial effects of a chemical or surgical sympathectomy, whereas blockade of adrenoceptors may still be advantageous.

In conclusion, the findings of the present study indicate that loose ligation of a sciatic nerve in rats provokes denervation-induced arterial supersensitivity to catecholamines. This su persensitivity consists of impaired presynaptic reuptake of and postjunctional supersensitivity to catecholamines. A similar pathophysiological mechanism seemingly accounts for (arterial) sympathetic dysfunction in RSD patients. 


\section{Chapter 14}

SUMMARY AND GENERAL DISCUSSION 
Reflex sympathetic dystrophy (RSD) is a neuropathic pain syndrome usually localised in an extremity and often resulting from trauma. Although many underlying pathophysiological mechanisms have been proposed, the exact pathogenesis of RSD is still unclear. Consequently, RSD is often not recognised and treated inappropriately or with delay, patients may undergo various types of treatment which frequently have little rational basis. It is therefore not surprising that the results of these types of treatment are often not satisfactory.

It was not until 1988 , before an animal model of partial peripheral nerve injury was developed in which signs and symptoms could be induced reminiscent of those observed in RSD (chronic constriction injury (CCI) $)^{40}$.

The presence of spontaneous and stimulus-induced pain in RSD patients indicates high levels of discharge from many of the injured nerve's afferents towards the central nervous system. Evidence in support of the latter hypothesis has been provided by electrophysiologic studies in $\mathrm{CCl}$ rats ${ }^{260.562}$. In contrast, although signs and symptoms observed in RSD patients as well as CCI rats suggest that partial nerve injury simultaneously affects efferent function of sympathetic and antidromically acting sensory nerve fibres, little experimental evidence has been provided outlining these effects.

It was the first and principal aim of this thesis to clarify, in both RSD patients and in $\mathrm{CCI}$ rats, the effects of partial nerve injury on the activity and sensitivity of the sympathetic nervous system. More specifically, we wanted to clarify whether sympathetic dysfunction consists of either an afferently-induced increase in efferent sympathetic nerve impulses or of denervation-induced supersensitivity to (circulating) catecholamines. Besides, in case of denervation-induced supersensitivity to catecholamines, we wanted to elucidate whether this phenomenon consists of prejunctional (i.e., deviation) supersensitivity or of postjunctional (i.e., adaptive) supersensitivity. A second aim of this thesis was to clarify the mechanism underlying the observed phenomenon of (partial) sympathetic denervation. The latter may result from interruption of sympathetic efferents at the site of nerve injury or from dysfunction of sympathetic neurons at the level of the central nervous system and/or paravertebral sympathetic ganglia. A third aim was to clarify the effects of partial nerve injury on efferent function of antidromically acting primary sensory nerve fibres. More specifically, we wanted to clarify whether this type of nerve injury provokes antidromic (i.e., directed towards the periphery) impulses in these nerve fibres with consequent release of vasodilator neuropeptides at their peripheral nerve endings.

\subsection{VALIDITY OF THE CCI MODEL}

For reasons discussed elaborately in chapter 2 of this thesis, we opted to refrain from using the more traditional than useful separation between RSD and causalgia patients. Hence, within the frame-work of this thesis we have studied patients with proven injury of a large peripheral nerve as well as patients with soft-tissue injury (i.e., injury of peripheral nerve twigs). In contrast, the clinical symptomatology of CCI rats is always provoked by partial injury of a large peripheral nerve. This discrepancy necessitates careful consideration in extrapolating the results from studies in CCl rats to the clinical condition of RSD. However, it is likely that at least part of the RSD patients, in which injury of a large peripheral nerve could not be demonstrated 
electrophysiologically or clinically, suffered from nerve injury anyway: it has been demonstrated in rats that an inflammation of the sclatic nerve sheath can produce hyperalgesia in the ipsilateral hind paw whereas electron-microscopy does not show axonal damage ${ }^{354}$. Besides, signs and symptoms induced by nerve injury at different sites may be mediated by similar or overlapping pathophysiological mechanisms. The observation that injury to a large mixed peripheral nerve causes also degeneration of terminal nerve twigs distal to the site of injury $^{499}$ argues in favour of this hypothesis. An additional argument in favour of this hypothesis is that signs and symptoms in rats with partial injury of a large (mixed) peripheral nerve are alike those observed in RSD patients without established injury of a large (mixed) peripheral nerve.

A second discrepancy which necessitates careful consideration in extrapolating the results from studies in CCI rats to the climical condition of RSD is that not in all RSD patients, studied within the framework of this thesis, signs and symptoms resulted from compression injury. However, it has been demonstrated that chromic gut ligatures laid next to instead of being placed around the sciatic nerve also produce behavioural evidence of neuropathic pain ${ }^{354}$ " This finding indicates that, in the $\mathrm{CCI}$ model, not only compression but also a disturbance of the micro-milieu contributes to the injury of the sciatic nerve. Hence, this model may be relevant not only to nerve compression injury but to any clinical condition in which the micromilieu of neural tissue is altered (e.g., due to trauma or inflammation).

Various observations argue in favour of the hypothesis that the CCI model is a valid model to study pathophysiological mechanisms underlying RSD. Stimulus-induced as well as stimulus-independent sensory abnormalities in CCI rats are identical to those observed in RSD patients. Likewise, CCI rats develop motor, autonomic, and trophic disturbances which are also observed: in RSD patients. Besides, in this thesis it was demonstrated that the pattern of microcirculatory changes in CCI rats is in line with the pattern of microcirculatory changes in RSD patients.

\subsection{SYMPATHETIC DYSFUNCTION}

The presence of autonomic and possibly also of trophic disturbances in RSD patients ${ }^{528}$ and CCI rats ${ }^{40}$ indicates that partial nerve injury may alter the activity of the sympathetic nervous system. Moreover, several observations suggest that this altered activity of the sympathetic nervous system is involved in the development and/or maintenance of sensory abnormalities. Firstly, circumstances which increase the activity of the sympathetic nervous system (e.g., cold, stress) generally aggravate sensory abnormalities ${ }^{71,419}$. Secondly, interruption of the activity of the sympathetic nervous system generally reduces pain ${ }^{41,382}$.

\section{2.a Nature of sympathetic dysfunction}

Although it is generally agreed that dysfunction of the sympathetic nervous system contributes to the clinical symptomatology of RSD, the exact nature of sympathetic dys function remains enigmatic. So far, sympathetic dysfunction mostly has been purported to consist of an increase in efferent sympathetic nerve impulses. According to this theory, any type of nerve or softtissue injury increases the level of sensory input to the spinal cord and thus the activity of the 
internuncial netron pool. The latter may stimulate anterolateral sympathetic efferents, as a result of which the level of efferent sympathetic nerve impulses from the spinal cord to the affected extremity could be increased (somato-sympathetic reflex) ${ }^{57,333}$. Evidence in support of this hypothesis was obtained from clinical observations that surgical or chemical sympathectomy may relieve sensory abnormalities and improve reduced skin blood flow in RSD patients ${ }^{41,231,545}$ as well as in experimental animal models of partial nerve injury $39,281,382$.

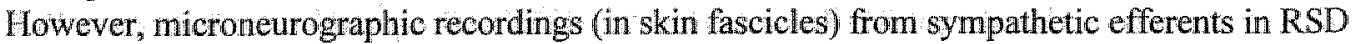
patients do not exhibit increased activity ${ }^{71,43,513}$. This finding argues against an afferentlyinduced increase in the level of efferent sympathetic nerve impulses. Consequently, we hypothesised that sympathetic dysfunction in RSD patients consists of supersensitivity to catecholamines consequent to (partial) sympathetic denervation.

The main finding of the studies presented in this thesis is that sympathetic dysfunction distal to the site of nerve injury consists of (both prejunctional and postjunctional) supersensitivity to catecholamines consequent to (partial) sympathetic denervation.

\section{Sympathetic denervation}

(Partial) sympathetic denervation was considered to be present in RSD patients, distal to the site of the precipitating event, on the basis of several observations. Firstly, the sympathetically mediated vasoconstrictor response to dependency at the thermoregulatory level of skin microcirculation was impaired at all 3 clinical stages of RSD (chapter 6). This disturbance progressed with clinical stage and duration of RSD. Since postural vasoconstriction is abolished after local nervous blockade by lidocaine ${ }^{219}$ as well as after sympathectomy ${ }^{219}$, this finding strongly suggests the presence of (partial) sympathetic denervation which increases with clinical stage and duration of RSD. Our finding that the disturbance of the vasoconstrictor response to dependency was more pronounced at the sympathetically controlled thermoregulatory level than at the non-neurally controlled nutritive level additionally argues in favour of the presence of (partial) sympathetic denervation. Secondly, stimulation of intramural adrenergic nerves in subcutaneous arteries that had been isolated from amputated extremities of stage III (chronic cold sensation) RSD patients demonstrated reduced vasoconstrictor responses which indicates functional sympathetic denervation of these bloodvessels (chapter 13). Our finding of sympathetic denervation in the affected extremities of RSD patients is in line with the results of various other studies in which it was demonstrated that venous plasma levels of noradrenaline ${ }^{138,218}$ and neuropeptide $Y^{137}$ collected in the affected limb of RSD patients are decreased when compared with plasma samples obtained from the contralateral (unaffected) extremity.

In line with the findings in RSD patients, various observations in this thesis indicate that, distal to the site of nerve injury, efferent sympathetic nerve activity in the affected limb of $\mathrm{CCI}$ rats is reduced rather than increased. Firstly, the sympathetically mediated vasoconstrictor response to cooling of the rat abdomen was impaired from an early stage after nerve injury onwards (chapter 10). Since this vasoconstrictor response has been demonstrated to be impaired in rats subjected to lumbar sympathectomy ${ }^{328}$, this finding strongly suggests the presence of ongoing (partial) sympathetic denervation. Secondly, subcutaneous arteries that had been isolated from the hind paw of rats with 3 weeks old CCI demonstrated reduced adrenergic nerve fibre density as well as impaired contractile responses to (electrical) stimulation of 
intramural adrenergic nerves (chapter 1.3). These findings are indicative of, respectively, structural and functional (partial) sympathetic denervation of these blood vessels. Besides, presynaptic reuptake of noradrenaline was impaired, which is also indicative of (partial) sympathetic denervation. Our finding of sympathetic denervation in the affected limbs of CCI rats is in line with the results of various other studies in which it was demonstrated that this type of nerve injury provokes axonal degeneration of small unmyelinated (most likely also sympathetic) nerve fibres in the loosely ligated sciatic nerve ${ }^{31,69,208}$. In these studies, axonal degeneration of small unmyelinated nerve fibres varied from $20 \%{ }^{376}$ to as much as $84 \% \%^{69}$. Besides, our findings confirm previous observations of Wakisaka et al ${ }^{533}$, who also demonstrated noradrenergic depletion in plantar hind paw vessels of CCI rats.

\section{Supersensitivity to catecholamines}

Supersensitivity to catecholamines was considered to be present in RSD patients, distal to the site of the precipitating event, on the basis of several observations. Firstly, sensitivity to noradrenaline was found to be increased in arteries isolated from subcutaneous fat of amputated extremities of stage III (chronic cold sensation) RSD patients (chapter 13). Secondly, skin blood flow at the mainly sympathetically controlled thermoregulatory level of skin microcirculation was increased at stage I and reduced at stages II and III of the RSD syndrome (chapter 5). As alluded to before, the sympathetically mediated vasoconstrictor response to dependency of the affected extremity was found to be impaired at all 3 clinical stages of RSD which is indicative of ongoing (partial) sympathetic denervation. Since sympathetic denervation causes supersensitivity of vascular structures to catecholamines ${ }^{42,23,3}$, the observed decrease in skin blood flow at stages II/III is also indicative of supersensitivity to catecholamines. The finding on supersensitivity to catecholamines is in line with observations of Arnold and colleagues ${ }^{19}$, who demonstrated that veins in limbs affected by RSD are supersensitive to noradrenaline.

In line with the findings in RSD patients, various observations in CCI rats attest to the presence of supersensitivity to catecholamines distal to the site of nerve injury. Firstly, sensitivity to noradrenaline was found to be increased in subcutaneous arteries isolated from the hind paw of rats with 3 weeks old CCI (chapter 13). Secondly, skin blood flow developed from an increase at an early stage after nerve injury to a decrease at a later stage (chapter 10). As alluded to before, the sympathetically mediated vasoconstrictor response to cooling of the rat abdomen was found to be impaired from an early stage after loose sciatic nerve ligation onwards, which is indicative of ongoing (partial) sympathetic denervation. Since sympathetic denervation causes supersensitivity of vascular structures to catecholamines ${ }^{42,22.3}$, the observed decrease in skin blood flow at a later stage after the CCI also suggests the presence of supersensitivity to catecholamines.

Supersensitivity to catecholamines may result from increased postjunctional responsiveness, a phenomenon referred to as adaptive supersensitivity and/or from impaired prejunctional reuptake, a phenomenon referred to as deviation supersensitivity ${ }^{164}$. The results of the study in $\mathrm{CCl}$ rats described in chapter 13 indicate that the phenomenon of supersensitivity to (circulating) catecholamines, as observed distal to the site of nerve injury, consists of both postjunctional supersensitivity to and impaired presynaptic retiptake of catecholamines. The presence of postjunctional (adaptive) supersensitivity to catecholamines 
was inferred from the increase in contractile responses to the $\alpha_{1}$-adrenoceptor agonist phenylephrine. Prejunctional (deviation) supersensitivity was indicated by the observation that exposure of the arteries to cocaine, which blocks presynaptic reuptake, significantly increased sensitivity for noradrenaline on the ligated, but not on the non-ligated side. Impairment of presynaptic reuptake of catecholamines may be related to degeneration of small unmyelinated (most likely also sympathetic) nerve fibres in the loosely ligated sciatic nerve $\mathrm{e}^{69,376}$.

It has been demonstrated previously that sympathetic denervation of vascular structures may increase sensitivity to catecholamines ${ }^{36,42,233,499}$. Hence, the phenomenon of supersensitivity to catecholamines most likely results from (partial) sympathetic denervation. The latter hypothesis is supported by the negative correlation between maximal contractile responses during electrical stimulation of intramural adrenergic nerve fibres and sensitivity to noradrenaline as observed in hind paw arteries from rats with 3 weeks old CCI (chapter 13).

\section{2.b Mechanisms underlying sympathetic denervation}

Partial sympathetic denervation of skin microvessels, as observed in RSD patients and CCI rats distal to the site of the precipitating event, may result from interruption of sympathetic efferents at the site of this event. However; the results of several studies suggest that, besides acute interruption of sympathetic efferents, other neuropathic mechanisms may be involved in the reduction of noradrenergic vasomotor innervation. Wakisaka et al ${ }^{533}$ demonstrated in the $\mathrm{CCl}$ model that (nearly) total noradrenergic depletion develops slowly over a period of about a month, whereas in the case of interruption of sympathetic efferents one would expect that noradrenergic depletion develops more rapidly. Besides, it has been demonstrated that noxious stimulation of nociceptors (without damaging sympathetic nerve fibres) may cause inhibition of skin vasoconstrictor activity $200,237,252$. Another mechanism involved in reduction of noradrenergic vasomotor innervation could be decreased synthesis of norepinephrine. The latter phenomenon has been demonstrated to result from both transection and crushing of a nerve ${ }^{74,263}$. Collectively, these observations indicate that partial sympathetic denervation may result not only from interruption of sympathetic efferents at the site of nerve injury, but also from dysfunction of sympathetic neurons at the level of the central nervous system and/or paravertebral sympathetic ganglia.

The findings of the studies presented in this thesis strongly suggest that partial sympathetic denervation distal from the site of the precipitating event predominantly results from interruption of sympathetic efferents at the site of this event. Besides, the findings of the studies in this thesis attest to the presence of reduced sympathetic vasoconstrictor outflow from the central nervous system and/or paravertebral sympathetic ganglia. On the clinically affected side, however, this effeet appears to be preponderated by the effect of somato-sympathetic reflexes. Consequently, the level of efferent sympathetic nerve impulses proximal to the site of the precipitating trauma is increased.

Evidence in favour of the hypothesis that partial sympathetic denervation distal to the site of the precipitating trauma predominantly results from interruption of sympathetic efferents at the site of nerve injury was inferred from the observation that an incongruity existed in sympathetic dysfunction between sites proximal to and distal to the precipitating trauma (chapter 9). Proximally, sympathetic nerve impulses appeared to be increased at all stages of the RSD syndrome. This finding suggests that nerve injury increases the level of sensory input 
to the spinal cord as a result of which anterolateral sympathetic efferents are stimulated and the level of efferent sympathetic nerve impulses from the spinal cord to the affected extremity is increased (somato-sympathetic reflex). In contrast, the findings distal to the site of the precipitating event are indicative of supersensitivity to catecholamines consequent to partial sympathetic denervation. This discrepancy between sites proximal and distal to the site of the precipitating event is indicative of interruption of sympathetic axons at the site of this event. Besides, this discrepancy may account for spatial variation in signs and symptoms of sympathetic dysfunction, which has been reported to occur in RSD patients. Our finding of an increased level of efferent sympathetic nerve activity proximal to the site of trauma is in line with the results of a study by McLachlan et $\mathrm{al}^{356}$, who demonstrated in the CCI model that following nerve injury noradrenergic perivascular axons sprout into dorsal root ganglia and form basket-like structures around large-diameter axotomised sensory neurons. The latter eould be activated repetitively by sympathetic stimulation which suggests that an important site of sympatho-sensory interactions and thus of abnormal discharges of sensory nerve fibres in the $\mathrm{CCI}$ model is the dorsal root ganglion. The functional importance of the latter mechanism was demonstrated by a study of Kajander et $\mathrm{al}^{26.1}$ who showed that in the CCl model the spontaneous discharges and sensitivity to $K^{+}$channel blockade seen in $A B$ and $A \delta$ primary afferents originate in the dorsal root ganglion and not at the injured region of the nerve.

A reduced sympathetic vasoconstrictor outflow from the central nervous system and/or paravertebral sympathetic ganglia towards the clinically unaffected extremity was inferred from the observation that the sympathetically mediated vasoconstrictor response to sympathetic stimuli is impaired in the clinically unaffected limb of stage I RSD patients (chapter 8) as well as $\mathrm{CCl}$ rats (chapter 11 ). This finding suggests dysfunction of sympathetic neurons at the level of the central nervous system and/or paravertebral sympathetic ganglia. However, on the clinically affected side, assessment of sympathetic dysfunction proximal to the site of the precipitating trauma indicated an increased level of efferent sympathetic nerve impulses at all 3 clinical stages of RSD (chapter 9). This discrepancy indicates that, on the clinically affected side, the effect of reduced sympathetic vasoconstrictor outflow from the central nervous system and/or paravertebral sympathetic ganglia is preponderated by an increased level of afferentlyinduced efferent sympathetic nerve impulses (somato-sympathetic reflexes).

\section{2.c Involvement of sympathetic nervous system in other signs and symptoms}

In this thesis, we report the presence of denervation-induced supersensitivity to catecholamines in skin microvessels distal to the site of nerve injury in both RSD patients and CCI rats. This supersensitivity phenomenon most likely accounts for the decrease in basal skin blood flow (reduced temperature and paleness of the skin) as observed at stages II/III of the RSD syndrome (chapter 5) as well as at later stages after nerve injury in CCI rats (chapter 10). In general, there is a delay in the appearance of adaptive supersensitivity, which in vascular structures may last from 3 days up to $2-4$ weeks ${ }^{164}$. Hence, on the basis of the findings presented in this thesis we can not exclude that the increase in skin blood flow at stage I of RSD results from sympathetic denervation. However, the findings reported in chapter 12 argue against the latter hypothesis: the increase in skin blood flow which was observed at day 4 after loose sciatic nerve ligation could be overruled by chemical blockade of sensory nerve fibres. Besides, subsequent blockade of non-sensory nerve fibres even further reduced skin blood flow. Collectively, these 
observations indicate that the increase in skin blood flow at this stage results from active vasodilation rather than from reduced sympathetic vasoconstrictor outflow from the central nervous system towards the affected limb.

It is likely that in cases of partial nerve injury non-vascular structures are simultaneously subjected to autonomic denervation and consequent supersensitivity to catecholamines. Since sympathectomy has been demonstrated to induce supersensitivity to catecholamines not only in vascular, but also in non-vascular structures ${ }^{148}$, denervation-induced supersensitivity to catecholamines may be involved in the development and/or maintenance of several other features of RSD.

Firstly, several lines of evidence suggest that the sympathetic nervous system is involved in the development and/or maintenance of sensory abnormalities: intravenous regional sympathetic blocks with guanethidine ${ }^{187,336,503}$, paravertebral sympathetic ganglion blockade $^{115,235}$, paravertebral sympathetic ganglionectomy ${ }^{403,560}$, and administration of $\alpha$ adrenoceptor blockers ${ }^{17,181}$ all have been reported to relief pain in RSD patients. Secondly, intradermal injection of norepinephrine rekindles the pain and hyperalgesia that had been relieved in RSD patients by sympathectomy ${ }^{544}$, but does not cause sensory abnormalities in healthy subjects. Thirdly, electrical stimulation of sympathetic efferents in nerve-injured patients has been reported to cause pain ${ }^{536}$. In this study, increased discharge rates to adrenaline and to sympathetic trunk stimulation were blocked by the $\alpha$-adrenoceptor blocker phentolamine, but not by the $B$-adrenoceptor blocker propranolol, indicating the involvement of $\alpha$-adrenoceptors. The cellular basis for this increased adrenergic sensitivity is unknown. One of the possibilities is that adrenoceptors are already present on axons of peripheral nerves but become effective not until the general excitability of these axons increases. The latter hypothesis seems unlikely because it implies that increased adrenergic sensitivity should always develop whenever the excitability of the peripheral axon is sufficiently increased. Alternatively, increased adrenergic sensitivity in cases of partial nerve injury may result from an increase in the number and/or responsiveness of axonal adrenoceptors in peripheral nerves. Although sofar no direct evidence has been presented for such an increase, the increase in $\alpha$-adrenoceptor synthesis, which has been reported to occur in the neuroma model ${ }^{357}$, argues in favour of this hypothesis. Moreover, Drummond et al ${ }^{139}$ demonstrated an increase in the number of $\alpha_{y}$ adrenoceptors in the hyperalgesic skin of RSD patients. Since arterioles, sweat glands, and hair follicles were excluded from analysis, it is likely that this finding is indicative of an increased number of $\alpha_{1}$-adrenoceptors in fine intra-epidermal nerve fibres. An increase in the number and/or responsiveness of axonal adrenoceptors in peripheral nerves may result from (partial) sympathetic denervation. The latter hypothesis is supported by several observations. Firstly, sympathectomy alone may induce spontaneous pain in the sympathectomised region ${ }^{332,423,457}$, whereas it is generally agreed that, under normal conditions, activity in the sympathetic nervous system has little effect on the excitability of primary afferent nociceptors ${ }^{467}$. Secondly, it has been demonstrated in the peripheral as well as the central nervous system that interfering with neurotransmission can lead to altered sensitivity and altered receptor binding in the effector neurons ${ }^{164}$. Two main hypotheses exist as to the localisation of upregulated adrenoceptors in peripheral nerves. Initially, it has often been purported that the upregulated adrenoceptor is localised on the primary afferent, and that stimulation of these adrenoceptors results in activation and/or sensitisation of the primary afferent ${ }^{105}$. However, the results of a recent study 
in rats suggest that the adrenoceptor is localised on a sympathetic postganglionic axon, the stimulation of which provokes release of prostaglandins which, in turn, mediate the activation and/or sensitisation of primary sensory afferents ${ }^{188}$.

Secondly, autonomic denervation of sweat glands may reduce sweat production, whereas consequent supersensitivity may provoke hyperhydrosis.

Thirdly, it has been demonstrated that the sympathetic nervous system is involved in epidermal cell growth and maturation ${ }^{396,455}$. Hence, sympathetic dysfunction may also account for trophic changes of the skin which are frequently observed at later stages of the RSD syndrome. Another factor which may be involved in the development of trophic skin changes is impairment of skin blood flow at the nutritive level of the skin microcirculation. In chapter 5 we report that skin blood flow at this level is reduced at stages I/III of RSD. This decrease, which confirms previous observations ${ }^{44,442}$, coincides with a decrease in total skin blood flow. This coincidence suggests constriction of arterioles supplying both thermoregulatory and nutritive subsystems on the basis of denervation-induced supersensitivity to catecholamines.

One should realise that differences in rapidity, between the aforementioned tissues, with which autonomic denervation induces supersensitivity to catecholamines may account for the clinical observation that a specific feature of RSD is indicative of sympathetic denervation, while another simultaneously present feature may already suggest supersensitivity to catecholamines (e.g. red, warm hand with hyperhydrosis).

\section{2.d Why does sympathectomy work (in part of the RSD patients)?}

Both surgical ${ }^{394,425,439}$ and chemical ${ }^{115,235 ; 555}$ sympathectomy may have beneficial effects on sensory abnormalities in RSD patients. Likewise, chemical sympathectomy has been demonstrated to alleviate sensory abnormalities in CCI rats ${ }^{382}$. These findings seemingly argue against the concept that sympathetic dysfunction in cases of partial nerve injury consists of denervation-induced supersensitivity to catecholamines. However, one should realise that the study reported in chapter 13 demonstrates considerable interindividual differences in the extent of sympathetic denervation, which was almost never complete. Hence, a chemical or surgical sympathectomy may even further reduce the amount of norepinephrine released (prejunctionally) into the synaptic cleft. This mechanism may account for the observation that, although efferent sympathetic nerve activity already seems to be decreased, surgical and chemical sympathectomy may relieve sensory abnormalities. Nevertheless, on the long run these sympathectomies may even further increase the level of supersensitivity to catecholamines. The latter phenomenon may account for the clinical observation that in part of the patients with nerve injury which are subjected to paravertebral sympathetic ganglion blockade relief of pain is only transient ${ }^{15,475}$. Another finding which may account for the beneficial effects of sympathectomy in part of the RSD patients is reported in chapter 9 . In this study, it was demonstrated that the level of efferent sympathetic nerve activity is increased proximal to the site of nerve injury at all 3 clinical stages of RSD. Such an increase may induce discharge of regenerating sprouts or damaged C-nociceptors through local release of

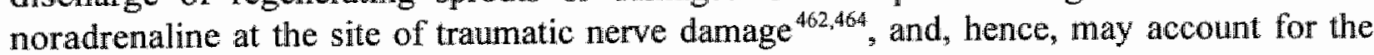
beneficial effects of sympathectomy. 


\subsection{ANTIDROMICALLY ACTING SENSORY NERVE FIBRES}

Several signs and symptoms commonly observed in RSD patients (such as dolour, calor, tumour, rubor, functio laesa) suggest the presence of an inflammatory process. Likewise, CCI rats demonstrate increased skin temperature at an early stage after nerve injury as well as reduced pain thresholds not only within but also outside the territory innervated by the injured sciatic nerve. It has been postulated by several authors that these inflammatory phenomena result from release of neuropeptides from distal terminals of primary afferent fibres ${ }^{73}$. Electrical stimulation, tissue damage, and local axon reflexes may all induce impulses in sensory nerve fibres which are directed towards the periphery. These impulses, which are being referred to as antidromic impulses, cause the release of neuropeptides which, in turn, may provoke inllammatory events such as vasodilation, enhancement of vascular permeability, and protein extravasation. Thus, noxious stimulation of the skin of man leads not only to a flare response (redness of skin), but also to edema and reduced pain thresholds both locally and more widespread $^{326}$.

\section{3.a Clinically affected side}

Skin blood flow in the affected hind paw of CCI rats develops from an increase at an early stage after nerve injury (day 3-5) to a decrease at a later stage (day 7-28) (chapter 10).

At day 4 , capsaicin application to the loosely ligated sciatic nerve (which selectively blocks axoplasmic transport of neuropeptides as well as impulse propagation in sensory nerve fibres) overruled the vasodilator response induced by loose ligation of the sciatic nerve (chapter 12). This indicates that antidromically acting sensory fibres in the injured sciatic nerve are involved in this early vasodilator response. Such a neurogenically mediated vasodilator response may involve antidromically acting unmyelinated $(C)$ as well as small myelinated $(A \delta)$ sensory nerve fibres ${ }^{255}$. Besides, subsequent blockade of non-sensory nerve fibres (by means of perineural application of lidocaine) at this time-point further reduced skin blood flow (chapter 12). This suggests that at this early stage non-sensory nerve fibres are also involved in the vasodilator response induced by partial nerve injury. Since so far no evidence has been presented for the involvement of other than sensory and sympathetic nerve fibres in neurogenic skin vasomotor control, the effect of blockade of impulse propagation of non-sensory nerve fibres most likely can be attributed to blockade of sympathetic nerve fibres. A sympathetically mediated vasodilator response may involve activation of (vasodilator) $\beta$-adrenoceptors. However, so far no direct evidence for the existence of B-adrenoceptors in rat skin is available, which suggests that another (sympathetically mediated) vasodilator mechanism must be involved. Such a mechanism may involve release of (vasodilator) neuropeptides at the endings of sympathetic nerve fibres. Although no direct evidence for the existence of such a mechanism is available, the observation that sympathetic ganglion cells of the cat contain mRNA coding for part of the mature substance P may argue in favour of this hypothesis ${ }^{329}$.

At day 21, perineural application of capsaicin further reduced skin blood flow, which indicates that in the CCI model antidromically acting sensory nerve fibres are involved in a vasodilator response not only at an early, but also at a later stage after ligation (chapter 12). Besides, our finding that subsequent blockade of non-sensory nerve fibres even further reduced 
skin blood flow suggests that these fibres are also involved in a vasodilator response at a later stage (chapter 12). As alluded to before, the latter mechanism most likely involves sympathetic nerve fibres. The vasodilator response at this later stage, however, is overruled by a nonneurogenic vasoconstrictor mechanism.

Our finding that antidromically acting sensory nerve fibres are involved in a vasodilator response at a later stage after $\mathrm{CCI}$ is in line with a study of Basile et $\mathrm{al}^{32}$. These authors found a decrease in the vasodilator response to $\mathrm{SP}$ and reduced plasma extravasation in response to $\mathrm{SP}$ in the clinically affected hind paw of CCI rats. The decreased vasodilator response to SP most likely results from decreased receptor numbers and/or effectiveness consequent to release of large amounts of SP from the peripheral endings of sensory nerves. This hypothesis is supported by the observation that SP levels in the spinal dorsal horn of CCI rats are decreased $^{38}$, suggesting increased release of SP at peripheral endings of sensory nerve libres.

\section{3.b Clinically unaffected side}

Electrical excitation of nociceptive afferents in an extremity has been demonstrated to increase skin blood flow in the contralateral extremity. Hence, one would expect that loose sciatic nerve ligation, which causes excitation of nociceptive afferents ${ }^{260,562}$, may also provoke a vasodilator response in the contralateral hind paw. On the nonligated side, such a response may involve inhibited skin vasoconstrictor activity as well as neurogenically mediated active vasodilation.

In chapter 11 we report that loose ligation of the rat sciatic nerve induces an increase in skin blood flow in the contralateral hind paw from day 3 after ligation onwards. Blockade of afferent input from the loosely ligated sciatic nerve to the spinal cord (by means of lidocaine) at day 5 postinjury completely interrupted this vasodilator response. On the non-ligated side, the vasodilator response seemingly involved inhibited skin vasoconstrictor activity as well as release of vasodilator neuropeptides at the periphery of antidromically acting sensory nerve fibres. The presence of inhibited skin vasoconstrictor activity was inferred from the observation that the sympathetically mediated vasoconstrictor response of skin microvessels to cooling of the rat abdomen was impaired from day 3 onwards (with the exception of day 14), which is indicative of ongoing reduced skin vasoconstrictor activity ${ }^{328}$. Moreover, the involvement of antidromically acting sensory nerve fibres in the vasodilator response on the non-ligated side was indicated by the observation that capsaicin application to the non-ligated sciatic nerve attenuates the vasodilator response induced by contralateral loose sciatic nerve ligation.

Our finding of antidromically acting sensory nerve fibres on the non-ligated side is also in line with the study of Basile et al ${ }^{32}$ who demonstrated a decrease in the vasodilator response to SP in the clinically unaffected hind paw of CCI rats.

\section{3.c Involvement of antidromically acting sensory nerve fibres in other signs and symptoms}

In paragraph $\Downarrow 4.2$, we have outlined the involvement of antidromically acting sensory nerve fibres in skin blood flow abnormalities observed in CCI rats on the ligated and non-ligated side. It is tempting to speculate that antidromically acting sensory nerve fibres also play a role in skin blood flow abnormalities observed in the clinically affected as wel $\rrbracket$ as the clinically unaffected extremity of RSD patients. 
Antidromically acting sensory nerve fibres provoke vasodilation through release of substances from their peripheral endings. This vasodilator response, however, is only one of a sequence of events which may result from noxious stimulation of noeiceptors and which lead to other signs characteristic of inflammation. A major contribution to this field was made by Lewis ${ }^{32,325}$ who investigated in detail the sequence of events induced by mechanical excitation of sensory nerve fibres (firmly stroking the human skin with a blunt instrument). The observed response was referred to as the triple response: 1) the red reaction due to local dilatation of skin microvessels, 2) the flare, a widespread dilatation of neighbouring arterioles brought about by a local axon reflex and accompanied by hyperalgesia, and 3) the wheal, i.e., local increased permeability of skin microvessels. Collectively, these signs and symptoms are being referred to as neurogenic inflammation. Following studies have demonstrated that not only mechanical but also chemical ${ }^{90}$ and electrical ${ }^{271}$ excitation of sensory nerve fibres somewhere at axonal level may also provoke neurogenic inflammation.

Substance $P$ and other neuropeptides are considered to be the cardinal mediators of neurogenic inflammation ${ }^{318}$. The neuropeptide substance $P$ exerts its vasodilator effect through direct interaction with endothelial cells ${ }^{15}$. Besides, substance $P$ binds to mast cells, thus causing degranulation and release of such inflammatory mediators as histamine ${ }^{274}$. Histamine acts indirectly on primary afferents to induce the release of other inflammatory mediators. Besides, it may exert a direct vasodilator effect on the microvasculature. Collectively, these mechanisms may account for effects of neuropeptides such as inducing vasodilation, increasing the excitability of primary sensory nerve fibres ${ }^{132}$, and enhancing the permeability of skin microvessels ${ }^{318}$.

As may be inferred from the aforementioned, it is likely that antidromically acting sensory nerve fibres, through release of substance $P$ and other neuropeptides from their peripheral endings, may be involved not only in vasodilation, but also in the development of other (inflammatory) features observed in RSD patients and/or CCI rats. Firstly, since substance $P$ and other neuropeptides may increase the excitability of primary sensory nerve fibres ${ }^{132}$, it is likely that release of these substances at the peripheral endings of sensory nerve fibres contributes to increased excitability of surrounding sensory nerve fibres and, hence, to sensory abnormalities. Secondly, the observation that activation of sensory nerve fibres provokes increased vascular permeability ${ }^{90}$ may account for edema formation, which is most pronounced at early stages of RSD ${ }^{528}$. In line with this clinical observation, histological evaluation of biopsies obtained from RSD patients showed edema formation in synovium ${ }^{294}$, muscle ${ }^{285}$, bloodvessel $\mathrm{s}^{285}$, and bone ${ }^{306}$. Thirdly, the second part of the triple response (flare; widespread dilatation of neighbouring arterioles accompanied by hyperalgesia) may account for the clinical observation that, in RSD patients, skin blood flow abnormalities as well as sensory abnormalities may spread out of the initial area of injury. This spread of signs and symptoms may be brought about by local axon reflexes and/or humoral distribution of inflammatory mediators.

In chapter 11 , we report that loose ligation of the rat sciatic nerve induces a vasodilator response in the contralateral hind paw, which, among others, involves antidromically acting sensory nerve fibres on the non-ligated side. Likewise, skin blood flow was found to be increased in the clinically unaffected limb of stage I RSD patients (chapter 8). The observation that partial nerve injury induces antidromic impulses in sensory nerve fibres in the contralateral 
extremity may account for the clinical phenomenon of "mirror maging". This phenomenon, which has been reported to occur in RSD patients ${ }^{294,463}$ as well as CCI rats ${ }^{23,280,474}$, refers to the development of minor signs and symptoms (skin blood flow abnormalities, sensory abnormalities, edema) in the clinically unaffected extremity. Similarly, Levine and colleagues ${ }^{221}$ demonstrated that a standard injury stimulus (subcutaneous injection of normal saline into the foot pad), which also activates nociceptive afferents, induces sensory abnormalities and increased vascular permeability in the contralateral hind limb of the rat. These contralateral responses, referred to as reflex neurogenic inflammation, also involves antidromically acting nociceptive afferents in the noninjured extremity ${ }^{32}$. The similarity between their and our findings suggests that similar mechanisms are involved in these contralateral responses.

\subsection{INTERACTIONS BETWEEN SYMPATHETIC AND SENSORY NERVOUS SYSTEM}

In this thesis, we have outlined how skin blood flow changes in RSD patients and CCI rats result from denervation-induced supersensitivity to catecholamines as well as from antidromically acting sensory nerve fibres. The former phenomenon causes a decrease in skin blood flow, whereas the latter phenomenon provokes an increase. Hence, the presence of these two opposing mechanisms may account for the clinical observation that skin blood flow abnormalities in RSD patients, especially at intermediate stages (stage II), may demonstrate short-term fluctuations ${ }^{58}$. These short-term fluctuations in skin temperature may result from a dynamic interplay between opposing vasomotor effects driven by vasoconstrictor sympathetic versus vasodilator antidromic mechanisms. E.g., factors which increase the level of circulating catecholamines (stress, cold) may rapidly reduce skin blood flow. In contrast, factors which increase the level of antidromic impulses in sensory nerve fibres (exercise) may rapidly increase skin blood flow.

Partial nerve injury has been demonstrated to increase the sensitivity of nociceptor-units to catecholamines. Besides, we have demonstrated that partial nerve injury induces antidromic impulses in sensory nerve fibres. These two phenomena may be causally related: the neurogenic inflammatory response evoked by partial nerve injury may be involved in the development of the increased sensitivity to catecholamines of nociceptor-units. The latter hypothesis is supported by a study of Drummond ${ }^{136}$. This author demonstrated that capsaicin application to the skin of healthy volunteers, which provokes an inflammatory response through release of neuropeptides from peripheral endings of antidromically acting sensory nerve fibres, causes heat hyperalgesia of the involved skin area. Moreover, heat hyperalgesia persisted in case of subsequent application of noradrenaline but not saline iontophoresis ${ }^{318}$. The increase in the level of somato-sympathetic interactions after capsaicin application may relate to the permeability increasing properties of neuropeptides; catecholamines which normally can not pass the blood-nerve barrier may become able to do so in case of increased microvascular permeability. 


\subsection{FUTURE DIRECTIONS}

\section{5.a Therapeutical interventions}

The observed phenomenon of denervation-induced supersensitivity to catecholamines suggests that blockade of adrenoceptors may have beneficial effects on sensory abnormalities induced by partial nerve injury. The latter is supported by a study of Kim and colleagues ${ }^{281}$ demonstrating that administration of phentolamine relieves signs of allodynia in nerve-injured rats. Phentolamine ${ }^{17}$ and phenoxybenzamine ${ }^{180}$ were reported to have beneficial effects on sensory abnormalities in humans with partial nerve injury as well An advantage of blockade of adrenoceptors over chemical or surgical sympathectomy could be that the long term results of the latter may be negatively influenced by a further increase in (super-) sensitivity to catecholamines. Besides, in cases where sympathetic denervation is already (sub-) complete as a result of the initiating event (nerve injury), one may expect few beneficial effects of sympathectomy, whereas blockade of (supersensitive) adrenoceptors may still be advantageous. A disadvantage of the aforementioned non-selective $\alpha$-adrenoceptor blockers is that they may have serious side-effects (such as hypotension) which may prohibit adequate dosage for pain relief. It is, however, likely that sensory abnormalities incluced by partial nerve injury are mediated by a subset of adrenoceptors. The latter hypothesis is supported by the observation that prazosin ${ }^{6}$ relieves sensory abnormalities in RSD patients, whereas propranolol has little effect ${ }^{420}$. Hence, these sensory abnormalities seemingly are $\alpha_{1}$-adrenoceptor mediated. Since prazosin has less side-effects than non-selective $\alpha$-adrenoceptor blockers, future research should focus on the effectiveness of selective $\alpha_{1}$-adrenoceptor blockers in relieving pain. Besides, further determination of the subtype of the $\alpha_{1}$-adrenoceptor involved in mediation of sensory abnormalities may yield the possibility of even more selective $\alpha$-adrenoceptor blockade. Hopefully, side-effects may thus be kept to a minimum while simultaneously adequate dosage for pain relief might become possible.

The findings in our study attest to the presence of a neurogenic vasodilator mechanism in the chronic constriction injury model. As alluded to before, such a neurogenic mechanism acts through release of neuropeptides from the peripheral endings of primary afferents. Neuropeptides have additionally been reported to increase the excitability of primary sensory nerve fibres ${ }^{132}$ and to increase vascular permeability ${ }^{318}$. Hence, in case of partial nerve injury, the alleged release of neuropeptides is most likely related not only to vasodilation, but also to other features of neurogenically mediated inflammation such as sensory abnormalities and edema formation, respectively. The latter concept may raise the possibility of new therapeutic approaches for patients with partial nerve injury. E.g., capsaicin; which provokes a profound and apparently irreversible abolition of the capability of sensory fibres to mediate a neurogenic inflammatory response ${ }^{85,62,250,317}$ may be applied perineurally or topically (to the affected skin). The effectiveness of topically applied capsaicin has been demonstrated previously in RSD patients $^{77}$. Alternatively, neuropeptide-receptor blockers (such as the substance $\mathrm{P}$ receptor antagonist D-Pro ${ }^{2}$, D-Phe ${ }^{7}$, D-Trp ${ }^{9}$ ) may prevent the development and/or maintenance of neurogenic inflammation induced by partial nerve injury.

The concept that RSD may result from compressive neuropathy may raise the possibility of decompressive interventions such as surgical decompression or intravenous administration of hyperosmolar fluids in order to reduce perineural and intraneural edema. Likewise, the 
concept that RSD may also result from alterations in the micro-milieu surrounding the nerve (due to trauma or inflammation) may render surgical transposition of the injured nerve a potentially beneficial therapeutical option.

\section{5.b Fundamental studies}

This thesis has provided insight into the nature of sympathetic dysfunction induced by partial nerve injury. However, one should realise that all experimental studies were performed on vascular structures. Since pain is the most troubling feature for most RSD patients, future studies should try to clarify whether this phenomenon of denervation-induced supersensitivity to catecholamines is also present in peripheral nerves. More specifically, the cellular basis for increased adrenergic sensitivity of these peripheral nerves should be explored in more detail. To this end, the subtype, number, and/or affinity of axonal adrenoceptors in partially injured peripheral nerves should be assessed by means of radio-immuno-assays. Besides, the exact localisation of upregulated adrenoceptors (i.e., on sympathetic or sensory axons) should be determined by means of immunohistochemistry. Electrophysiologic studies should be performed in order to find out which specific subtype adrenoceptor mediates activation and/or sensitisation of the primary afferent.

A second important finding of this thesis is that partial nerve injury provokes vasodilation in the ipsilateral hind paw through antidromic impulses in sensory nerve fibres. As alluded to before, it is likely that these antidromic impulses, which act through release of neuropeptides at the peripheral endings of sensory nerve fibres, additionally induce other features of neurogenic inflammation such as excitation of nociceptor nerve fibres, increased vascular permeability and increased leukocyte sequestration. The presence of the two latter phenomena in the CCI model has been demonstrated ${ }^{99}$. Future studies, both in RSD patients and $\mathrm{CCI}$ rats, should investigate whether partial nerve injury increases the release of neuropeptides at the peripheral endings of affected sensory nerve fibres. Moreover, it is of interest to clarify whether this increased release of neuropepticles is involved in the development and/or maintenance of sensory abnormalities. More specifically, it should be investigated which neuropeptide is involved. Besides, it is of interest to investigate how neuropeptides increase the excitability of nociceptors. One possibility is that the neuropeptideinduced increase in vascular permeability renders it possible for various substances (among others catecholamines) to cross the blood-nerve barrier and subsequently activate the nociceptor unit, whereas under physiologic circumstances these substances may not be able to do so.

\subsection{CONDENSED SUMMARY}

The main findings of this thesis are summarised in fig. 14.1. Partial nerve injury increases the number of impulses in sensory nerve fibres directed towards the central nervous system (CNS) as may be inferred from the presence of pain. This barrage of sensory impulses seemingly provokes several effects at the level of the CNS such as antidromic (vasodilator) impulses in sensory nerve fibres on the clinically unaffected side. Besides, this barrage of sensory impulses appears to induce a dualistic effect at the level of the CNS as far as the sympathetic nervous 
system is concemed: the level of sympathetic nerve impulses directed towards the clinically unaffected extremity appears to be reduced. On the clinically affected side (proximal to the site of nerve injury), however, the level of sympathetic impulses seems to be increased. This discrepancy may be explained as follows: an increased sensory input generally reduces sympathetic outflow. On the clinically affected side, the latter effect seems to be preponderated by somato-sympathetic reflexes. In contrast, on the clinically affected side distal to the site of nerve injury we observed (partial) sympathetic denervation. This discrepancy between sites proximal and distal to the nerve injury suggests that partial sympathetic denervation results from interruption of sympathetic axons at the site of partial nerve injury. Besides, this discrepancy may account for spatial variation in signs and symptoms of sympathetic dysfunction which has been reported frequently in RSD patients. This partial sympathetic de nervation mest likely also accounts for the phenomenon of supersensitivity to catecholamines as observed distal to the site of partial nerve injury. This supersensitivity consists of increased postjunctional sensitivity to as well as impaired presynaptireuptake of catecholamines. Besides increasing the level of sensory impulses directed towards the CNS, it was demonstrated in this thesis that partial nerve injury simultaneously provokes antidromic vasodilator impulses (i.e., directed towards the periphery) in sensory nerve fibres. One should realise that the aforementioned events may result from partial nerve injury at midaxonal level as well as at the level of the peripheral nerve twigs.

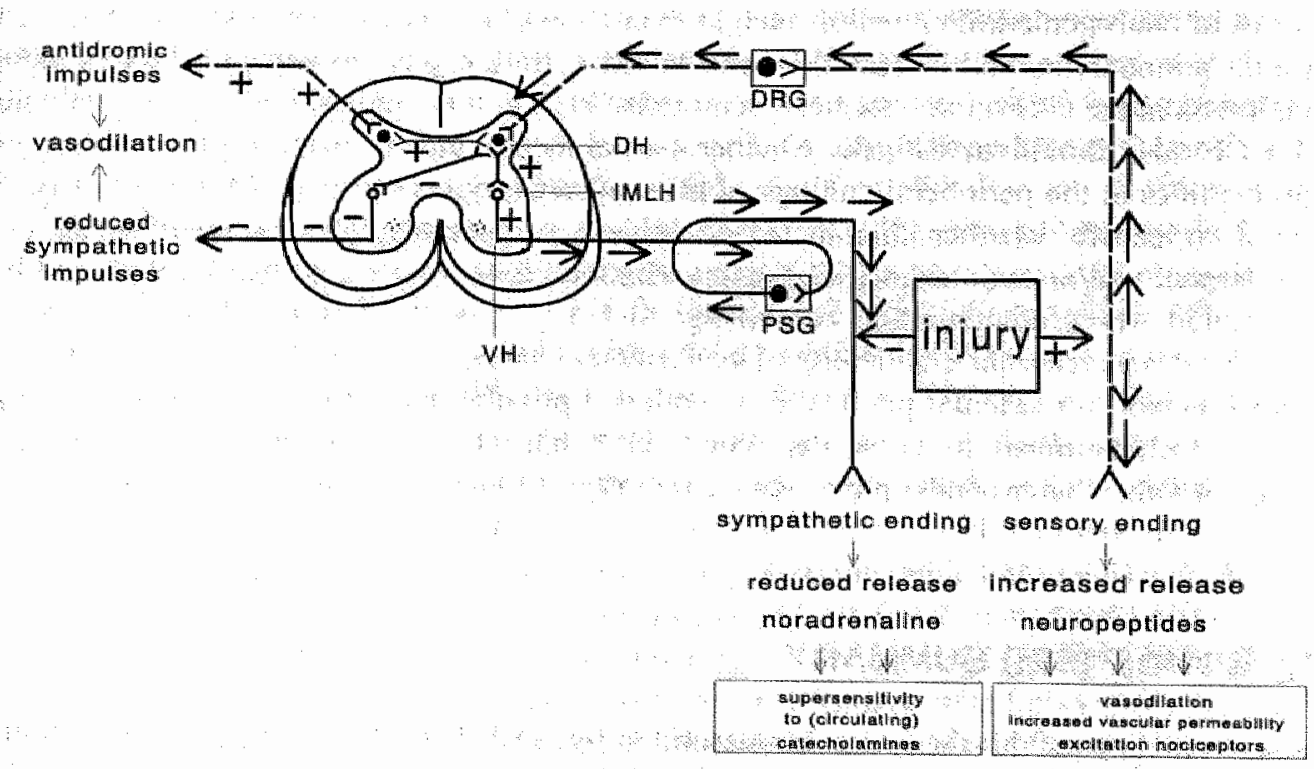

Fig. 14.1: effects of partial nerve injury on function of sympathetic and sensory nerve fibres. For detalled explanation, see condensed summary (paragraph 14.6 ). 


\section{REFERENCES}

1. Algodystrophy [editorial]. Br Med J 1978; 1: 461-462.

2. Posttraumatische dystrofie. Nijmegen Post-Academisch Ondenwijs Geneeskunde Katholieke Universiteit Nijmegen, 1992.

3. Classification of Chronic Pain: Descriptions of Chronic Pain Syndromes and Definition of Terms. Seattle: IASP Press, 1994

4. Aanonsen LM, Kajander KC, Biennett GJ, Seybold VS. Autoradiographic analysis of 125 I-substance P binding in rat spinal cord following chronic constriction injury of the sciatic nerve. Brain Res 1992; 596 : 259-268.

5. Aanonsen $L M$, Wilcox $G L$. Nociceptive action of excitatory amino acids in the mouse: effects of spinally administered opioids, phencyclidine and sigma agonists. J Pharmacol Exp Ther 1987; 243: 9-19.

6. Abram SE, Lightfoot RW. Treatment of long-standing causalgia with prazosin. Reg Anesth 1981; 6: 79-81.

7. Abram SE, Yaksh TL. Systemic lidocaine blocks nerve injury-induced hyperalgesia and nociceptor-driven spinal sensitization in the rat. Anesthesiology 1994; 80: 383-391.

8. Aftimos S. Reflex neurovascular dystrophy in children. N Z Med J 1986; 99: 761-763.

9. Aguayo A Nair CPV, Midgley R. Experimental progressive compression neuropathy in the rabbit. Arch Neurol 1971: 24: $358-364$

10. Albert J, Waldburger $M$. [Algodystrophy in the chronic stage following treatment with phenobarbital]. Schweiz Med Wochenschr 1983; 113:470-473.

11. Allen G, Samson B. Contralateral Horner's syndrome following stellate ganglion block. Can Anaesth Soc J 1986; 33: 112-113.

12. Amadio PC, Mackinnon SE, Merritt WH, Brody GS, Terzis JK. Reflex sympathetic dystrophy syndrome: consensus report of an ad hoc committee of the American Association for Hand Surgery on the definition of reflex sympathetic dystrophy syndrome. Plast Reconstr Surg 1991;87;371-375.

13. Ameratunga $R$, Daly $M$, Caughey DE. Metastatic malignancy associated with reflex sympathetic ystrophy. J Rheumatol $1989,46,406-407$,

14. An HS, Hawthorne KB, Jackson WT. Reflex sympathetic dystrophy and cigarette smoking. J Hand Surg Am 1988: 13: 458-460:

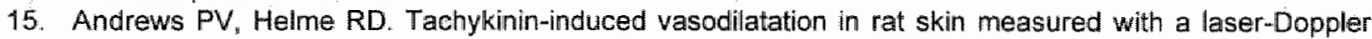
flowmeter: evidence for receptor-mediated effects. Regul Pept 1989; 25: $267-275$.

16. Ardid D, Guilbaud $G$. Antinociceptive effects of acute and "chronic" injections of tricyclic antidepressant drugs in a new model of mononeuropathy in rats. Pain 1992; 49: 279-287.

17. Amer S. Intravenous phentolamine test: diagnostic and prognostic use in reflex sympathetic dystrophy. Pain $1991 ; 46: 17-22$.

18. Arner S, Meyerson BA. Lack of analgesic effect of opioids on neuropathic and idiopathic forms of pain [see comments]. Pain 1988; 33: 11-23.

19. Arnold JM, Teasell RW, MacLeod AP, Brown JE, Carruthers SG. Increased venous alpha-adrenoceptor responsiweness in patients with reflex sympathetic dystrophy. Ann Intern Med 1993; 118: 619-621

20. Asch L, Kuntz Jl., Meyer R. [Postinfectious algodystrophy]. Rev Rhum Mal Osteoartic 1984; 51: 337-340.

21. Atkins RM, Duckworth T, Kanis JA. Features of algodystrophy after Colles' fracture. J Bone Joint Surg Br 1990; $72: 105-110$.

22. Attal $N$, Chen $Y L$, Kayser $V$, Guilbaud $G$. Behavioural evidence that systemic morphine may modulate $a$ phasic pain-related behaviour in a rat model of peripheral mononeuropathy. Pain 1991; 47: 65-70.

23. Attal $N$, Jazat $F$, Kayser $V$, Guilbaud $G$. Further ewidence for 'pain-related' behaviours in a model of unilateral peripheral mononeuropathy. Pain 1990; 41: 235-251.

24. Attal $N$, Kayser $V, G$ Guibaud $G$. The bidirectional dose-dependent effect of systemic naloxone is also related to the intensity and duration of pain-related disorders: a study in a rat model of peripheral mononeuropathy.

Brain Res 1990; 525: 170-17.4

25. Bach FW, Jensen TS, Kastrup J, Stigsby B, Dejgard A. The effect of intravenous lidocaine on nociceptive processing in diabetic neuropathy [see comments]. Pain 1990; 40: 29-34.

26. Baranowski $R$, Lynn B, Pini A. The effects of locally applied capsaicin on conduction in cutaneous nerves in four mammalian species. Br J Pharmacoll 1986; 89: 267-276.

27. Barber FA, Click J, Birtt BT. Complications of ankle arthroscopy. Foot Ankle 1990; 10: 263-266.

28. Barbut $D$. Polak JM, Wall $P D$. Substance $P$ in spinal cord dorsal horn decreases following peripheral nerve injury. Brain Res 1981; 205: 289-298.

29. Barolat $G$, Schwartzman $R$, Woo $R$. Epidural spinal cord stimulation in the management of reflex sympathetic dystrophy. Stereotact Funct Neurosurg 1989; 53:29-39. 
30. Barowsky $\mathrm{EI}_{2}$ Zweig $\mathrm{JB}$, Moskowitz J. Thermal biofeedback in the treatment of symptoms associatied with reflex sympathetic dystrophy. J Child Neural 1987; 2: 229-232.

34. Basbaum $A l_{i}$ Gautron $M_{1}$ Jazat $F_{n}$ Mayes $M$, Guilbaud $G$. The spectrum of fiber loss in a model of vieuropathic pain in the rat an electron microscopic study. Pain 1991, 47:359-367.

32. Basile $S_{i}$ Khalli $Z$, Helme RD. Skin vascular reactivity to the neuropeptide substance $P$ in rats with peripheral mononeuropathy. Pain $1993 ; 52: 217-222$.

33. Baumbach GL, Heistad DD, Siems JE. Effects of sympathetic nerves on composition and distensibility of cerebral arterioles in rats. J Physilol 1989; 416: 123-140.

34. Beacham WS, Perl ER. Characteristics of a spinal sympathetic reflex. J Physiol 1964; 173: 431-448.

35. Bej MD, Schwartzman RJ. Abnormalities of cutaneous blood flow regulation in patients with reflex sympathetic dystrophy as measured by laser Doppler fluxmetry. Arch Neurol 1991; 48: 912-915.

36. Benarroch $E$. Schmelzer JD, Ward KK, Nelson DK, Low PA. Noradrenergic and neuropeptide $Y$ mechanisms in guanethidine-sympathectomized rats. Am J Physiol 1990; 259: $371-375$.

37. Bennett $G J$ Experimental models of painful peripheral neuropathies. News in Physiological Sciences 1990; 5: $128-133$.

38. Bennett $G J_{1}$ Kajander $K C$, Sahara $Y$, ladarola $M J$, Sugimoto $T$. Neurochemical and anatomical changes in the dorsal horn of rats with an experimental painful peripheral neuropathy. In: Cervero $F$. Bennett GJ, Headly $\mathrm{PM}_{\mathrm{t}}$ eds. Processing of sensory information in the superficial dorsal horn of the spinal cord, New York: Plenum, 1989: 453-471.

39. Bennett GJ, Ochoa JL. Thermographic observations on rats with experimental neuropathic pain. Pain 1991; 45: $61-67$.

40. Bennett GJ, Xie YK. A peripheral mononeuropathy in rat that produces disorders of pain sensation like those seen in man. Pain 1988; $33: 87-107$.

41. Betcher AM, Bean G, Casten DG. Continuous procaine block of paravertebral sympathetic ganglions. JAMA $1953 ; 151: 288-292$.

42. Bevan JA, Tsuru H. Long term denervation of vascular smooth muscle causes not only functional but also structural change. Blood Vessells 1979; 16: 109-112

43. Bickerstaff $D R$, Kanis JA. Algodystrophy: an under-recognized complication of minor trauma. $\mathrm{Br} J$ Rheumatol $1994 ; 33: 240-248$.

44. Bieber EJ, Linscheid RL, Dobyns JH, Beckenbaugh RD. Failed distal ulna resections. J Hand Surg Am 1988; 13: $193-200$.

45. Bini G, Cruccu G, Hagbarth KE, Schady W, Torebjork E. Analgesic effect of vibration and cooling on pain induced by intraneural electrical stimulation. Pain 1984; 18:239-248.

46. Birkenfeld B. Erfahrungen mit der Echinacim-Therapie beim Sudeckschen Syndrom. Therapie der Gegenwart $1954 ; 93: 425$.

47. Blanchard J, Ramamurthy $S_{1}$ Walsh $\mathbb{N}_{1}$ Hoffman J, Schoenfeld L. Intravenous regional sympatholysis: a double-blind comparison of guanethidine, reserpine, and normal saline. J Pain Symptom Manage 1990 ; 5: $357-361$.

48. Blinn $\mathrm{G}$, Heinz $\mathrm{G}$, Jurna $\mathrm{I}$. Effects of substantia nigra stimulation on suralis evoked spinal reflex activity: Comparison with the effects of morphine and stimulation in the periaqueductal gray matter. Neuropharmacology $1980 ; 19 ; 75-85$.

49. Blumberg $\mathrm{H}$. [Development and treatment of the pain syndrome of reflex sympathetic dystrophy: Clinical picture, experimental investigations and new pathologicall considerations]. Der Schmerz 1988; 2: 125-143.

50. Blumberg $H$, Griesser $H J$, Hornyak M. Neurologilc aspects of clinical manifestations, pathophysiology and therapy of reflex sympathetic dystrophy (causalgia, Sudeck's disease). Nervenarzt 1991; 62: 205-211.

51. Blumberg $H$, Griesser $H J$, Hornyak M. Distal post-traumatic edema-symptom af a sympathetic reflex dystrophy (Sudeck's disease)? Z Orthop 1992; 130:9-15.

52. Blumberg $H$. Janig $W$. Changes of reflexes in vasoconstrictor neurons supplying the cat hindlimb following chronic nerve lesions: a model for studying mechanisms of reflex sympathetic dystrophy? I Auton Nerv Syst $1983 ; 7: 399-411$.

53. Blumberg $H$, Wallin $B G$. Direct evidence of neurally mediated vasodilatation in hairy skin of the human foot. J Physiol 1987; 382: 105-121.

54. Boas RA, Covino BG, Shahnarian A. Analgesic responses to i.v. lignocaine. $\mathrm{Br} J$ Anaesth 1982 ; 54 : $501-505$.

55. Bonelli $S_{\text {, Conoscente }} F_{1}$ Movillia $P G$, Restelli L. Francucci $B$, Grossi Ex Regional intravenous guanethidine vs. stellate ganglion block in reflex sympathetic dystrophies: a randomized trial. Pain 1983; 16: $297-307$.

56. Bonica JJ. The Management of Pain. Philadelphia: Lea \& Febiger, 1953.

57. Bonica JJ. Causalgia and other reflex sympathetic dystrophies. Postgrad Med 1973: 53: 143-170. 
58. Bonica JJ. Causalgia and other Reflex Sympathetic Dystrophies In Bonica JJ Liebeskind JC, Albe-Fessrad DG, eds. Advances in pain research and therapy, New York: Raven Press, 1979: 141-166.

59. Bonica $\mathrm{J}$ J. Causalgia and other sympathetic reflex dystrophies. In: Bonica $\mathrm{Jl}$, ed. The management of pain, 2nd ed. Philadelphia: Lea and Febiger, 1990: 220-243.

60. Bonnett $J$. Post-traumatische dystrophie, een klinische studie over symptomatologie, pathogenese en therapie der vegetatieve reacties na extremiteitenletsels. Amsterdam: Thesis, 1953.

61. Bos GMJ, Slaaf DW, Majoor GD, Tangelder GJ. Reneman RS. A model for chronic study of wascular morphology and reactivity of physically intact rat skin microcirculation. Int J Microcirc : Clin Exp 1993; 1 : 145-155.

62. Böhler L. Ist das Sudeck-Syndrom nach geschlossenen Verletzungen eine unabwendbare Unfallfolge oder eine vermeidbare Behandlungfolge. Langenbecks Arch Chir Suppl II Verh Dtsch Ges Forsch Chir 1956; 284: $43-53$.

63. Buch $\mathrm{J}_{\text {, Blauensteiner } \mathrm{W}}$, Scherafati $\mathrm{T}$, Vischer $\mathrm{HM}$, Fischer $\mathrm{W}$. Conservative treatment of calcaneus fracture versus repasitioning and percutaneous bore wire fixation. A comparison of 2 methods]. Unfallchirurg 1989; 92: 595-603.

64. Buechel FF, Pappas MJ, Iorio LJ. New Jersey low contact stress total ankle replacementu biomechanical rationale and review of 23 cementless cases. Foot Ankle 1988; 8: 279-290.

65. Buhring M. [Reflex dystrophy following so-called whiplash injury of the cervical spine] $z$ Orthop 1984; 122 : 281-286.

66. Burton Rl. Complications following surgery on the basal joint of the thumb. Hand Clin 1986; $2: 265-269$.

67. Campbell $U_{N}$, Raja SN, Meyer RA, Mackinnon SE. Myelinated afferents signal the hyperalgesia associated with nerve injury. Pain 1988; 32:89-94.

68. Carli $P_{\text {in }}$ Chagnon $A_{n}$ Elizagaray $A_{s}$ Paris J. [Algodystrophy of the hip secondary to coxitis caused by brucellosis]. Rev Rhum Mal Osteoartic 1990; 57:841-843.

69. Carlton SM, Dougherty PM, Pover $\mathrm{CM}_{\text {; }}$ Coggeshall RE. Neuroma formation and numbers of axons in a rat model of experimental peripheral neuropathy. Neuroscil Lett 1991; 131: 88-92.

70. Carron $\mathrm{H}, \mathrm{McCue} F$. Reflex sympathetic dystrophy in a ten year old. South Med J 1972; 65: 631-632.

71. Casale R, Elam M. Normal sympathetic nerve activity in a reflex sympathetic dystrophy with marked skin vasoconstriction. J Auton Nerv Syst 1992; 41: 215-219.

72. Casale $R$, La Rovere MT. Increased sympathetic tone in the left arm of patients affected by symptomatic myocardial ischemia. Funct Neurol 1989; 4: 161-163.

73. Chapman LF, Ramos AO, Goodell H, Wolff HG. Neurohumoral features of afferent fibers in man. Arch Neuroll 1961; 49: 49-8.2.

74. Cheah TB, Geffen LB. Effects of axonal injury on norepinephrine, tyrosine hydroxylase and monoamine oxidase levels in sympathetic ganglia. J Neurobiol $1973 ; 4: 443-452$.

75. Chen YL, Kayser V, Guilbaud G. Physical dependence develops without tolerance on repetitive low doses of morphine: study in normal and mononeuropathic rats. Pain 1990; S470(Abstract).

76. Cherington $M$, Happer I, Machanic B, Parry L. Surgeny for thoracic outlet syndrome may be hazardous to your health. Muscle Nerve 1986; 9: 632-634.

77. Cheshire WP, Snyder CR. Treatment of reflex sympathetic dystrophy with topical capsaicin. Gase report. Pain 1990; 42: 307-311.

78. Cho DS, Cho MJ. The electrodiagnosis of the carpal tunnel syndrome. S D J Med 1989; 42:5-8.

79. Chodoroff B, Ball RD. Lumbosacral radiculopathy, reflex sympathetic dystrophy and tarsal tunnel syndrome: an unusuall presentation. Arch Phys Med Rehabil 1985; 66: 185-1.87.

80. Choi Y, Yoon YW, Na HS, Kim SH, Chung JM. Behavioral signs of ongoing pain and cold allodymia in a rat model of neuropathic pain. Pain 1994; 59: 369-376.

81. Chow J, Schenck RR. Early continuous passive movement in hand surgery. Curr Surg 1989; 46: 97-100.

82. Christensen $K_{r}$ Jensen $E M_{i}$ Noer I. The reflex dystrophy syndrome response to treatment with systemic corticosteroids. Acta Chir Sicand 1982; 148: 653-655.

83. Chuinard RG, Dabezies EJ, Gould JS, Murphy GA, Matthews RE. Intravenous reserpine for treatment of reflex sympathetic dystrophy. South Med J 1981; 74: 1481-1484.

84. Chung JM, Choi $Y$, Yoon YW. Na HS. Effects of age on behavioral signs of neuropathic pain in an experimental rat model. Neurosci Lett 1995; 183: 54-57.

85. Chung JM, Lee $\mathrm{KH}$, Hori $Y$, Willis WD. Effects of capsaicin applied to a peripheral nerve on the responses of primate spinothalamic tract cells. Brain Res 1985; 329: 27-38.

86. Churcher MD. Algodystrophy after aortic bifurcation surgery. Lancet 1984, 2: 131-133.

87. Clark LC. Monitor and contral of blood and tissue oxygen tensions. Trans Am Soc Artif Intern Organs 1956; 2: $41-48$ 
88. Clere D, Salliere D, Bisson M, Massias P. [Association of algodystrophy and hyperparathyroidism]. Rev Med Interne $1983 ;$ : 159

89. Cline $\mathrm{MA}_{\mathrm{e}}$ Ochoa $\mathrm{J}$, Torebjork $\mathrm{HE}$. Chronic hyperalgesia and skin warming caused by sensitized $\mathrm{C}$ nociceptors. Brain $1989 ; 112: 621-647$.

90. Coderre TJ, Basbaum AI Levine JD. Neural control of vascular permeability: interactions between primary afferents, mast cells, and sympathetic efferents. Neurophysiol 1989, 62:48-58.

91. Coggeshall RE, Dougherty PM, Pover CM, Carton SM, Is llarge myellinated fiber loss associated with hyperalgesia in a model of experimental peripheral neuropathy in the rat? Pain 1993; 52: 233-242.

92. Constantinesco $\mathrm{A}$, Brunot $\mathrm{B}$, Demangeat $\mathrm{JL}$, Foucher $\mathrm{G}$, Farcot $\mathrm{JM}$. Three phase bone scanning as an aid to early diagnosis in reflex sympathetic dystropthy of the hand. A study of eighty-nine cases. Ann Chir Main $1986,5,93-104$.

93. Cooke ED, Ward C. Vicious circles in reflex sympathetic dystrophy--a hypothesis: discussion paper. $\mathbf{J} \mathbf{R}$ Soc Med $1990,83.9699$.

94. Cooper IS. Clinical and physiologic implications of thalamic surgery for disorders of sensory communication. 1. Thallamic surgery for intractable pain. J Neurol Sci $1965 ; 2: 493-519$.

95. Creutzig $A_{i}$ Dau $A$, Caspary $L$, Alexander $K$. Transcutaneous oxygen pressure measured at two different electrode core temperatures in healthy volunteers and patients with arterial occlusive disease. Int $J$ Microcirc : Clin Exp 1993; 5: 373-380.

96. Cronier $P$, Talha $A_{i}$ Toulemonde JL, Jaeger $F$, Guntz M. [Results of distraction by way of external metacarporadial fixation in fracture of the distal end of the radius. Apropos of 97 cases]. $\mathrm{J}$ Chir Paris 1991; 128: 8-12.

97. Culberson JL, Haines DE, Kimmel DL, Brown PB. Contralateral projection of primary afferent fibers to mammalian spinal cord. Exp Neurol 1979; 64: 83-97.

98. Culp WU, Ochoa $J_{1}$ Cline $M$, Dotson $R$. Heat and mechanical hyperalgesia induced by capsaicin: Cross modality threshold modulation in human C nociceptors. Brain 1989; 112: 1317-1331.

99. Daemen MARC, Kurvers HAJM "Slaaf DW, et al. Neurogenic inflammation in a model of neuropathic pain; in vitro assessment: Proceedings 8th world congress on pain, Vancouver, British Columbia, Canada 1996; 26(Abstract).

100. Daemen MJAP. De Mey JGR. Regional heterogeneity of arterial structural changes. Hypertension 1995; 24: $464-473$.

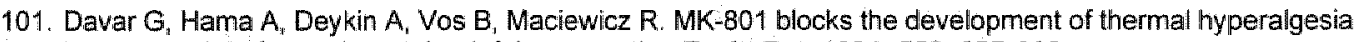
in a rat model of experimental painful neuropathy. Brain Res 1991: 553: 327-330.

102. Davidoff $G$, Morey $K$, Amann $M$, Stamps $J$. Pain measurement in reflex sympathetic dystrophy syndrome. Pain 1988; 32: 27-34

103. Davies J, Evans RH, Jones AW, Smith DA, Watkins JC. Differential activation and blockade of excitatory amino acid receptors in the mammalian and amphiblan central nervous systems. Comp Biochem Physiol C. $1982 ; 72: 211-224$.

104. Davies $J$, Watkins JC. Role of excitatory amino acid receptors in mono- and polysynaptic excitation in the cat spinal cord. Exp Brain Res: 1983; 49: 280-290.

105. Davis KD, Treede $R D$, Raja $S N$, Meyer RA, Campbell JN Topical application of clonidine relieves hyperalgesia in patients with sympathetically maintained pain. Pain 1991; 47: 309-317.

106. De Mey JG, Uitendaal MP, Boonen HC, Schiffers PM, Fazzi GE. Growth responses in isolated elastic muscular and resistance-sized arterial segments of the rat. Blood Vessels 1991; 28: 372-385.

107. De Mey JGR, Defreyn G, Lenaers A, Calderon P, Roba J. Arterial reactivity, blood pressure, and plasma levels of atrial natriuretic peptides in normotensive and hypertensive rats: effects of acute and chronic administration of atriopeptin III. J Cardiovasc Pharmacol 1987; 9: 525-535.

108. de Weerdt CJ, Journee HL, Hogenesch RI, Beks. JW. Sympathetic dysfunction in patients with persistent pain after prolapsed disc surgery. A thermographic study. Acta Neurochir Wien 1987; 89: 34-36.

109. Delcambre $B_{n}$ Cocheteux $P$, Piette $F$, Houcke $M$, Duquesnoy $B$. [Vasculitis following guanethidine block treatment in algodystrophy of the foot]. Rev Rhum Mal Osteoartic 1984; 51: 425-426.

110. DeLeo JA, Coombs DW. Willenbring $S$, et al. Characterization of a neuropathic pain model: sciatic cryoneurolysis in the rat. Pain $1994 ; 56: 9-16$

111. Dellon AL. Pitfalls in interpretation of electrophysiological testing. In: Gelberman RH, ed. Operative nerve repair and reconstruction, Philadelphia: JB Lippincott Co, 1991: 185-196.

112. Demangeat $ل_{L_{n}}$ Constantinesco $A$, Brunot $B$, Foucher $G$, Farcot JM. Three-phase bone scanning in reflex sympathetic dystrophy of the hand. J Nucl Med 1988; 29: 26-32.

113. Dequeker $J_{1}$ Geusens $P_{1}$ Verstaeten $A_{\|}$De Roo $M$. Vertebral crush fracture syndrome and reflex sympathetic dystrophy. Bone 1986; $7: 89-94$

114. DeTakats G. Reflex dystrophy of the extremities. Arch Surg 1937; 34: 939. 
115. DeTakats $G$. The nature of painful vasodilatation in causalgic states. Arch Neurol 1943; 50: 318-326.

116. DeTakats $G$. Causalgilic states in war and peace. JAMA 1945; 128: 699-704.

117. Devor M. Nerve pathophysiology and mechanisms of pain in causalgia d Auton Nerv Syst $1983 ; 7$ : $371-384$

118. Devor M. Nerve pathophysiology and mechanisms of pain in causalgit. $\mathrm{J}$ Auton Nerv Syst 1983; 7 : $371-384$.

119. Devor $M$. The pathophysiology and anatomy of damaged nerve. In: Wall PD, Melzack $R_{s}$ eds. Textbook of pain 2 nd ed Edinburgh: Churchill Livingstone, $1989: 63-81$.

120. Devor $M_{4}$ Govrin Lippmann $R_{n}$ Frank $I$, Raber P. Proliferation of primary sensory neurons in adult rat dorsal root ganglion and the kinetics of retrograde cell loss after sciatic nerve section. Somatosens Res $1985 ; 3$ : 139-167.

121. Devor $M$, Janig $W$, Michaelis $M$. Modulation of activity in dorsall root ganglion meurons by sympathetic activation in nerve-injured rats. J Neurophysiol 1994; $71: 38-47$.

122. Devor $M$, Lomazov $P_{\text {, Matzner } O}$. Sodium channel accumulation in injured axons as a substrate for

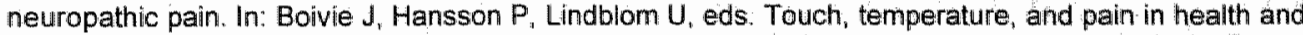
disease: mechanisms and assessments, progress in pain research and management, Seattle: IASP Press, 1994: 207-230.

123. Devor $M$, Raber $P$. Heritability of symptoms in an experimental model of neuropathic pain. Pain $1990 ; \mathbf{4 2}$ $51-67$.

124. Devor $M_{r}$ Wall PD. Cross-excitation in dorsal root ganglia of nerve-injured and intact rats. J Neurophysiol $1990 ; 64: 1733-1746$.

125. Dirksen $R$, Rutgers MJ, Coolen JM. Cervical epidural steroids in reflex sympathetic dystrophy. Anesthesiology $1987 ; 66: 71-73$.

126. Dougherty PM, Garrison CJ, Carltan SM. Differential influence of local anesthetic upon two models of experimentally induced peripheral mononeuropathy in the rat. Brain Res 1992; 570: 109-115.

127. Doupe J $J_{\text {. Cullen }} \mathrm{CH}$, Chance GQ. Posttraumatic pain and the causalgia syndrome. J Neurol Neurosurg Psychiatry 1944; $7: 33-48$.

128. Doury $P$, Dirheimer $y$, Pattin $S$. Algodystrophy. Diagnosis and therapy of a frequent disease of the locomotor apparatus. Berlin, Heidelberg, New York: Springer 1981.

129. Doury $P$, Pattin $S$, Eulry $F$, Leloire $O, A$ boulkrat $P$, Rolland $Y$. [Fatigue fractures of the femaral neck followed by algodystrophy]. Rev Rhum Mal Osteoartic $1987 ; 54: 425-427$

130. Daury P. Pattin S, Metges PJ, Eulry F, Barnoud D, Wendling D. [An uncommon case of spontaneous pseudofracture of the patella in algodystrophy]. Rev Rhum Mal Osteoartic 1987; 54: 499-500.

131. Draisci $G$, Kajander KC, Dubner $R$, Benneft $G J$, ladarola MJ. Up-regulation of opioid gene expression in spinal cord evoked by experimental nerve injuries and inflammation. Braim Res 1991; 560; 186-192.

132. Dray A, Urban L, Dickenson A. Pharmacology of chronic pain. TIPS 1994; 15: 190-197.

133. Drevet JG Carpentier $P$, Franco $A$, Phelip X. [Misleading pains in the lower limbs of rheumatologic origin]. Phlebologie 1985; 38: 255-263.

134. Driessen JJ, van der Werken $C$, Nicolai JP. Crul JF. Clinical effects of regional intravenous guanethidine (Ismelin) in reflex sympathetic dystrophy. Acta Anaesthesiol Sicand 1983; 27: 505-509.

135. Drucker WR, Hubay CA, Holden WD, Bukovnic JA. Pathogenesis of post-traumatic sympathetic dystrophy. Am J Surg 1959; 97: 454-465.

136. Drummond PD. Noradrenaline increases hyperalgesia to heat in skin sensitized by capsaicin. Pain 1995; 60: $311-315$.

137. Drummond PD, Finch PM, Edvinsson L, Goadsby PJ. Plasma neuropeptide $Y$ ' in the symptomatic limb of patients with causalgic pain. Clin Auton Res 1994; 4: 113-116.

138. Drummond $\mathrm{PD}$, Finch PM, Smythe GA. Reflex sympathetic dystrophy: the significance of differing plasma catecholamine concentrations in affected and unaffected limbs. Brain 1991; 114: 2025-2036.

139. Drummond PD, Skipworth S, Finch PM. Alpha-1 adrenoceptors in normal and hyperalgesic human skin. Clin Scil 1996; $91: 73-77$

140. Duncan KH Lewis RC.J, Racz G, Nordyke MD. Treatment of upper extremity reflex sympathetic dystrophy with joint stiffness using sympatholytic Bier blocks and manipulation. Orthopedics $1988 ; 11: 883-886$

141. Dunstan CR. Evans RA, Somers NM. Bone death in transient regional osteoporosis. Bone 1992; 13: 161-165.

142. Dyck PJ. Inwited review: limitations in predicting pathologic abnormality of nerves from the EMG examination. Muscle Nerve 1990; 13: 371-375.

143. Dyck PJ, Thamas PK, Lambert EH, Bunge R. Peripheral neuropathy. Philadelphia: Saunders, 1984

144. Echlin F, Owens FM, Wells WL. Observations on "Imajor' and 'minor" causalgia. Arch Neurol 1945; 62 $183-203$ 
145. Edeiken $J_{i}$ Wolferth $C C$. Persistent pain in the shoulder region following myocardial infarction. Am J Med $1936: 19: 201-210$.

146. Eerdmans $\mathrm{PH}$, Struyker Boudier HA, De Mey JGR. Contractile reactivity of isolated resistance arteries after 4 weeks of treatment with rilmenidine, clonidine, or hydralazine in spontaneously hypertensive rats. J Hypertens Suppl 1991, 9: S348-9.

147. Egle UT, Hoffmann SO Psychosomatic aspects of reflex sympathetic dystrophy: In: Stanton-Hicks M, Janig Wi Boas RA, eds. Reflex sympathetic dystrophy Boston: Kluwer, 1990 .

148. EK $B_{1}$ Lundgren $B$. Increased beta-adrenoceptor density after 6-hydroxydopamine pretreatment in rat colon and lung Acta Physiol Scand 1986, 127,455-460.

149. Erjavec $F$, Lembeck $F$, Florjanc Irman $T$, Skofitsch $G$, Donnerer-J, Saria $A$, Holzer $P$. Release of histamine by substance P. Naunyn-Schmiedeberg"s Arch Pharmac 1981:317.

150. Escobar PL. Reflex sympathetic dystrophy. Orthop Rev 1986; 15: 646-651.

151. Eulry $F$, Bauduceau $B$, Clement $R$, et al. [Algodystrophy and venous thrombosis of the lower extremities Diagnostic discussion. Apropos of 3 cases]. Phlebologle 1987; 40: 251-259.

152. Eulry $F$, Chevalier $X_{3}$ Crozes $P$, Prudat $M$, Lechevaller $D$, Larget Plet $B$. Is hyperlipidaemia a contributing factor to algodystrophy (reflex sympathetic dystrophy)? Cilin Rheumatol 1992; 11: 526-528.

153. Fagrell $B$. The relationship between macro- and microcirculation clinical aspects. Acta Pharmacol Toxicol Copenh $1986 ; 58$ Suppl 2:67-72.

154. Fahr LM, Sauser DD. Imaging of peripheral nerve lesions. Orthop Clin North Am 198:; 19: 27-41.

155. Fermaglich DR. Reflex sympathetic dystrophy in children. Pediatrics 1977; 60: 881-883.

156. Fialka V, Wickenhauser J, Engel A, Schneider B. [Sympathetic reflex dystrophy. Effectiveness of physical therapy treatment of Sudeck's syndrome]: Fortschr Med 1992; 110: 146-148.

157. Field $\mathfrak{J}$, Protheroe DL, Atkins RM. Algodystrophy after Colles fractures is associated with secondary tightness of casts. J Bone Joint Surg Br 1994; 76: 901-905.

158. Field J. Warwick $D$, Bannister $G C$. Features of algodystrophy ten years after Colles" fracture. J Hand Surg $\operatorname{Br} 1992 ; 17: 318-320$.

159. Fields HL. Can opiates relieve neuropathic pain? [letter]. Pain 1988; 35: 365-367.

160. Filliatreau $G$, Attal $N$, Hassig $R$, Guilbaud $G$, Desmeules J, DiGiamberardino L. Time-course of nociceptive disorders induced by chronic loose ligatures of the rat sciatic nerve and changes of the acetylcholinesterase transport along the ligated nerve. Pain 1994; 59: 405-413.

161. Fischman AJ, Rubin $\mathrm{RH}_{\mathrm{n}}$ Khaw BA, el al. Detection of acute inflammation with 111 ln-labeled nonspecific polyclonal IgG. Semin Nucl Med 1988: 18: 335-344.

162. Fitzgerald $M$, Woolf $C J$. The time course and specificity of the changes in the behavioural and dorsal horn celli responses to noxious stimuli following peripheral nerve capsaicin treatment in the rat. Neuroscience 1982; 7: 2051-2056.

163. Fleetwood Walker SM, Mitchell R, Hope PJ, Molony V, Iggo A. An alpha 2 receptor mediates the selective inhibition by noradrenaline of nociceptive responses of identified dorsal horn neurones. Brain Res 1985; 334: $243-254$

164. Fleming $W_{i}$ Westfall DP. Adaptive supersensitivity. In: Trendelenburg $U_{\text {" }}$ Weiner $N$, eds. Catecholamines I. Berlín Springer verlag, 1988: $509-559$.

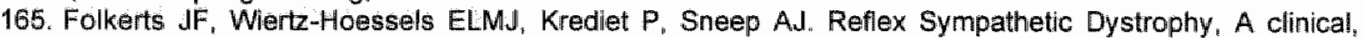
histological and experimental study. Confin Neurol 1969; 31: 145-175.

166. Ford $S R$, Forrest WHJ, Eltherington $L$. The treatment of reflex sympathetic dystrophy with intravenous regional bretylium. Anesthesiology 1988; 68: 137-140.

167. Francx $M$, Williame $L$, Herregods $P$, Heistercamp $F$, Chappel $R$. Reflex sympathetic dystrophy in hemiplegia: objectivation and evaluation using scintigraphy. Acta Belg Med Phys 1988; 11: 205-208.

168. Fraund $E_{1}$ Hattner HJ, Schroder $H$. Morbus Sudeck als Komplikation der Radiusfractur. Monatwchr Unfallheillkd 1970; 73: 569-574.

169. Fromm C, Deleo JA, Coombs DW, Colburn RW, Twitchell BB. The gangliaside GM1 decreases autotomy but not substance $P$ depletion in a peripheral mononeuropathy rat model. Anesth Analg 1993; 77: $501-506$.

170. Fruhstorfer $\mathrm{H}_{\mathrm{L}}$ Lindblom $\mathrm{U}$. Sensibility abnormalities in neuralgic patients studied by thermal and tactile pulse stimulation. In: Euler von U, Franzen $\mathrm{O}$, Lindblom U, Ottoson D, eds. Somatosensory mechanisms, London: Macmillan Press, 1984: 353-361.

171. Gainer MJ. Hypnotherapy for reflex sympathetic dystrophy. Am J Clin Hypn 1992; 34: 227-232.

172. Galeazza MT, Stucku CL, Jansen EM, Seybold VS. Analysis of CGRP and SP binding sites in rat spinal cord in an experimental model of acute, peripheral inflammation. Soc Neurosci Abst 1990; 16: 1073 (Abstract).

173. Gamse R, Petsche $U$, Lembeck $F$, Jancso $G$. Capsaicin applied to peripheral nerve inhibits axoplasmic transport of substance $P$ and somatostatin. Brain Res 1982; 239: 447-462. 
174. Gamse $R$, Saria $A$. Antidromic vasodilatation in the rat hindpaw measured by laser Doppler flowmetry: pharmacological modulation. J Auton Nerv Syst 1987; 19: 105-111.

175. Gautron $M_{i}$ Guilbaud $G$. Somatic responses of ventrobasal thalamic netrones in polyarthritic rats. Brain Res 1982; 237: 459-471.

176. Gautron $M$, Jazat $F$, Ratinahirana $H$, Hauw JJ, Guilbaud $G$. Alterations in myelinated fibres in the sciatic nerve of rats after constriction: possible relationships between the presence of abinormal small myelinated fibres and pain-related behaviour. Neurosci Lett 1990; 111:28-33.

177. Gburek $Z$. Results of treatment of reflex algodystrophy of the limbs with propranolol-Polfa and griseofulvin]: Reumatologia $1983 ; 21: 271-275$.

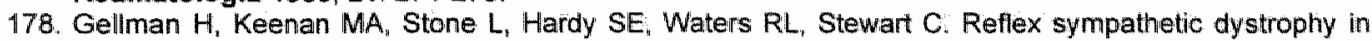
brain-injured patients. Pain 1992; 51: 307-311

179. Genant HK, Kozin F, Bekerman $C$, McCarty DJ, Sims $J$. The reflex sympathetic dystrophy syndrome. A comprehensive analysis using fine-detail radiography, photon absorptiometry, and bone and joint scintigraphy. Radiology $1975 ; 117: 21-32$.

180. Ghostine SY, Comair YG, Tumer DM, Kassel NF, Azar CG. Phenoxybenzamine in the treatment of causalgia. Report of 40 cases. J Neurosurg 1984; 60: 1263-1268.

181. Ghostine SY, Comair YG, Turner DM, Kassell NF, Azar CG. Phenoxybenzamine in the treatment of causalgia. Report of 40 cases. J Neurosurg 1984;60: 1263-1268.

182. Gibbons $\mathrm{J} J$, Wilson PR, Lamer TJ, Elliott BA. Interscalene blocks for chronic upper extremity pain. Clin $J$ Pain 1992; 8: 264-269:

183. Gillespie $\mathrm{JH}_{4}$ Menk EJ, Middaugh RE. Reflex sympathetic dystrophy: a complication of interscalene black. Anesth Analg 1987; 66: 1316-1317.

184. Ginzburg $R_{1}$ Seltzer $Z$. Subarachnoid spinal cord transplantation of adrenal medulla suppresses chronic neuropathic pain behavior in rats. Brain Res 1990; 523 : 147-150.

185. Gissen AJ; Covino BG, Gregus J. Differential sensitivities of mammalian nerve fibers to local anesthetic agents. Anesthesiology 1980; 53: 467-474.

186. Glick EN: Reflex dystrophy (algoneurodystrophy): results of treatment by corticosteroids. Rheumatol Phys Med 1973; 12: 84-88.

187. Glynn CJ, Basedow RW, Walsh JA Pain relief following post-ganglionic sympathetic blockade with I.V. guanethidine: Br J Anaesth 1981; 53: 1297-1302.

188. Gold MS, White DM, Ahlgren $\mathrm{SC}_{\mathrm{n}}$ Guo M, Levine JD. Catecholamine-induced mechanical sensitization of cutaneous nociceptors in the rat. Neurosci Lett 1994; 175: 166-170.

189. Goldberg MI, Kennedy SF. Reflex sympathetic dystrophy--recognition and management in gynecologic oncology. Gynecol Oncol 1979; 8: 288-295.

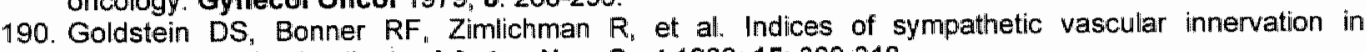
sympathectomized patients. J Auton Nerv Syst 1986; 15: 309-318.

191. Goltz F. Uber gefaberweiternde Nerve. Pflugers Arch 1874; 9: 174-197.

192. Goodman RR, Brisman R. Treatment of lower extremity reflex sympathetic dystrophy with continuous intrathecal morphine infusion. Appl Neurophysiol 1987" 50: 425-426.

193. Goris RJ. Treatment of reflex sympathetic dystrophy with hydroxyl radical scavengers. Unfallchirurg 1985; 88: $330-332$.

194. Goris RJ, Dongen LM, Winters HA. Are toxic axygen radicals involved in the pathogenesis of reflex sympathetic dystrophy? Free Radic Res Commun 1987; 3: 13-18.

195. Goris RJA. Conditions associated with impaired oxygen extraction. In: Gutierrez $\mathrm{G}$, Vincent $\mathrm{JL}$, eds. Tissue oxygen utilization, Berlin: Springer Verlag, 1991: 350-369.

196. Goris RJA, Kolkman WFA, Leenen LPH, Bebber van IPT, Corstens FHM, Heerschap A. Symptomatologie van posttraumatische dystrofie. In: Es van J, Joossens JV, Mandema E, Olthuis G, eds. Het Medisch Jaar, Utrecht: Bohn Scheltema Holkema, 1988: 165-177.

197. Goris RJA, Reynen JAM, Veldman PHJM. De posttraumatische distrofie. In: Mourik van JB, Patka P, eds. Letsels van de enkel en de voet. Epidemiologie, diagnostiek, therapie en revalidatie, Harem: Symposiumcommissie Chirurgie Nederland, 1990: 435-446.

198. Gracely $\mathrm{RH}$, Lynch $S A$, Bennett GJ. Painful neuropathy: altered central processing maintained dynamically by peripheral input. Pain 1992; 51: 175-194.

199. Greipp ME. Thomas AF. Familial occurrences of reflex sympathetic dystrophy. Clin J Pain 1991; 7 : 48 (Abstract).

200. Grosse $M$, Janig $W$. Vasoconstrictor and pilomotor fibres in skin nerves to the cat's tail. Pflugers Arch 1976; 361: 221-229.

201. Grosslight KR, Rowlingson $J_{C}$, Boaden RW. Herpes zoster and reflex sympathetic dystrophy. Anesth Analg 1986; 65 : 309-311. 
202. Grundberg $A B$, Reagan DS. Compression symdromes in reflex sympathetic dystrophy. $J$ Hand Surg $A m$ $1991 ; 16: 731-736$.

203. Guenee $B$, Tallet F, Raichvarg $D$, et al. Type IV hyperfipoproteinemia in patients with algodystrophy, Clin Exp Rheumatol $1985 ; 3 ; 49-52$.

204. Guilbaudd $G_{i}$. Benoist $\mathrm{JM}_{*}$ Gautron $M$. Electrophysiological evidence that morphine can exert an antinociceptive effect in a neuropathic state: a study in the ventrobasal thalamus of rats after moderate ligation of one sclatic nerve Brain Res $1991,551: 346-350$.

205. Guilbaud $G$, Benoist JM, Gautron Mi: Contribution of the sciatic and saphenous nerve to the ventrobasal thalamic neuronal responses to pinch in rats with a chronic sciatic nerve constriction: a study using anesthetic blocks and nerve section. Neuroscl Lett 1995; 187:197-200.

206. Guilbaud $G$, Benoist $J M$, Jazat $F$, Gautron $M$. Neuronal responsiveness in the ventrobasal thalamic complex of rats with an experimental peripheral mononeuropathy. J Neurophysiol $1990 ; 64: 1537-1554$.

207. Guillbaud $G$, Benoist $J M$, Levante $A$, Gautron $M$, Willer JC. Primary somatosensory cortex in rats with pain-related behaviours due to a peripheral mononeuropathy after moderate ligation of one sciatic nerve: neuronal responsivity to somatic stimulation. Exp Brain Res 1992; 92: 227-245.

208. Guilbaud $G$, Gautron $M$, Jazat $F$, Ratinahirana $H$, Hassig $R$, Hauw $J J$. Time course of degeneration and regeneration of myelinated nerve fibres following chronic loose ligatures of the rat sciatic nerve: can nerve lesions be linked to the abnormal pain-related behaviours? Pain 1993; 53: 147-158.

209. Gulbaud $G$, Kayser V, Benoist JM, Gautron M. Modifications in the responsiveness of rat ventrobasal thalamic neurons at different stages of carrageenin-produced inflammation. Brain Res 1986; 385: 86-98.

210. Guntheroth WG, Chakmakjian S, Brena SC, Ricketts HJ, Wiederhielm CA. Posttraumatic sympathetic dystrophy. Dissociation of pain and vasomotor changes. Am J Dis Child $1971 ; 121: 511-514$.

211. Guyton AC. Textbook of medical physiology. Philadelphia, London, Toronto: W.B. Saunders Company, 1991.

212. Hama AT, Sagen J: Reduced pain-related behavior by adrenal medullary transplants in rats with experimental painful peripheral neuropathy. Pain 1993; 52: 223-231.

213. Handwerker HO, Holzer-Petsche $\mathrm{U}$, Heym C, Welk E. C-fibre functians after topical application of capsaicin to a peripheral nerve and after neonatal capsalcin treatment. In: ChahI LA, Scolcsanyi J, Lembeck F, eds. Aintidramic vasodilatation and neurogenic inflammation, Budapest: Akademia Kiado, 1984: 57-78.

214. Hanna $\mathrm{MH}_{3}$ Peat $\mathrm{SJ}$. Ketanserin in reflex sympathetic dystrophy. A double-blind placebo cantrolled cross-over trial Pain 1989; 38: 145-150.

215. Hannington Kiff JG. Relief of Sudeck's atrophy by regional intravenous guanethidine. Lancet 1977; may: 1132-1133.

216. Hannington Kiff JG. Hyperadrenergic-effected limb causalgia: relief by IV pharmacologic norepinephrine blockade [letter]. Am Heart J 1982; 103: 152-153.

217. Hao $\sqrt{ } X_{n}, X u X J$, Aldskogius $H_{*}$ Seiger $A$, Wiesenfeld Hallin $Z$. Allodynia-like effects in rat after ischaemic spinal cord injury photochemically induced by laser irradiation. Pain 1991; 45: 175-185.

218. Harden RIN, Duc TA, Williams TR, Colley $D$ " Cate JC, Gracely RH. Norepinephrine and epinephrine levels in affected versus unveffected limbs in sympathetically maintained pain. Clin J Pain 1994; 10:324-330.

219. Hassan AAK, Rayman G, Tooke JE. Effect of indirect heating on the postural control of skin blood flow in the human foot. Clin Sci 1986; 70: 577-582.

220. Hassan AAK, Tooke JE. Mechanism of the postural vasoconstrictor response in the human foot. Clin Scil $1988 ; 75: 379-387$.

22\%. Hoerschap $A$, den Hollander JA, Reynen $H$, Goris RJ. Metabolic changes in reflex sympathetic dystrophy: a 31P NMR spectroscopy study. Muscle Nerve 1993; 16: 367-373.

222. Heims CA, O'Brien ET, Katzberg RW. Segmental reflex sympathetic dystrophy syndrome. Radiology 1980 ; 135: $67-68$.

223. Henriksern O. Local reflex in microcirculation in human subcutaneous tissue. Acta Radiol 1976; 98 : $447-456$.

224. Henriksen O. Sejrsen P. Local reflex in microcirculation in human cutaneous tissue. Acta Physiol Scand 1976: $98: 227-231$.

225. Henry JL. Substance P and pain. In: Euler von VS, Pernow B, eds. Substance P, New York: Raven Press, 1977: $231-240$.

226. Henry JL. Relation of substance P to pain transmission: neurophysiological evidence. Ciba Found Symp $1982 ; 206-224$.

227. Herrmann $L G$, Reineke $H G_{\text {" }}$ Caldwell JA. Post-traumatic painful osteoporosis: a clinical and roentgenological entity. Am J Roentgenol 1995.

228. Hobelmann CFJ, Dellon AL. Use of prolonged sympathetic blockade as an adjunct to surgery in the patient with sympathetic maintained pain. Microsurgery 1989; 10; 151-153. 
229. Hodges DL; McGuire TJ. Burning and pain after injury. is it causalgia or reflex sympathetic dystrophy? Postgrad Med 1988; 83: 185-192.

230. Hoeks AP, Brands PJ, Smeets FA, Reneman RS. Assessment of the distensibility of superficial arteries: Ulitrasound Med Biol 1990; 16: 121-128.

231. Hoff C, Kurvers HAJM, Ubbink DT, Jakimowicz J, Buth J, Jacobs M.JHM. Thoracoscopic sympathectiomy for reflex sympathetic dystrophy; clinical and microcirculatory results; long term follow-up. Surgical Endoscopy $1994 ; 8: 960$ (Abstract).

232 Hofstra $L$, Willigers $J M$, Huvers $F C$, et al. Short-term variation in the elastic properties of a muscular artery in humans. Clin Sei Colch 1994; 86: 567-574.

233. Hogestatt ED, Hammarstrom LE, Andersson KE, Holmin T. Contractile effects of various vasoactive agents in small rat portal veins and hepatic arteries and the influence of sympathetic denervation on the noradrenaline response. Acta Physiol Scand 1986; 128: 309-315.

234. Holden WD. Sympathetic dy strophy. Arch Surg 1948; 57:373384:

235. Homans J. Minor causalgia: A hyperesthetic neurovascular syndrome. N Engll J Med 1940; 222: $870-874$.

236. Hord $\mathrm{AH}_{4}$ Rooks MD, Stephens $\mathrm{BO}$, Rogers HG, Fleming LL. Intravenous regional bretylium and lidocaine for treatment of reflex sympathetic dystrophy: a randomized, double-blind study: Anesth Analg 1992; 74: $818-821$.

237. Horeyseck $G$, Janig $W$. Reflexes in postganglionic fibres within skin and muscle nerves after noxious stimulation of skin. Exp Brain Res 1974; 20: 125-134.

238. Horowitz SH. Peripheral nerve injury and causalgia secondary to routine venipuncture Neurology $1994 ;$ 44: $962-964$.

239. Huaux JP, Sonnet J, Bigaignon $G$, Nagant de Deuxchaisnes $C$. [Lyme arthritis and reflex sympathetic dystrophy. An uncommon but logical association] Rev Rhum Mal Osteoartic 1988; 55: 781-783:

240. Huber $A$, Wurm $G$, Witzmann $A$, Fischer J. [Percutaneous lumbar thermal sympathectomy: method, indications and results]. Neurochirurgia Stuttg 1992; 35: 85-88.

241. Huch $R$, Huch $A$, LUbbers DW. Trancutaneous pO2. Stuttgart, New York: Georg Thieme Verlag, Thieme-Stratton Inc. 1981;

242. Hugues FC, Munera $Y$, Le Jeunne C. [Unusual indications for beta-blockers]. Therapie 1985; 40; 417-4:21.

243. Hyland WT. Treating reflex sympathetic dystrophy with transdermal nitroglycerin. Plast Reconstr Surg 1989; 83: 195

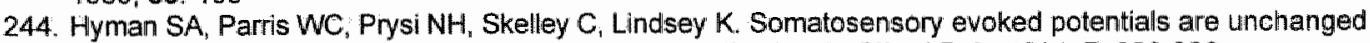
by reflex sympathetic dystrophy and by stellate ganglion block. Clin J Pain $1994 ; 7: 226-229$.

245. Imhalz BPM, Settels $\mathrm{J} J$, Meiracker van der $\mathrm{AH}$, Wesseling $K \mathrm{KH}_{3}$, Wieling $\mathrm{W}$. Non-invasive continuous finger blood pressure measurement during orthostatic stress compared to intra-arterial pressure. Cardiovasc Res 1990; 24: 214-221.

246. Ingram GJ, Scher RK, Lally EV. Reflex sympathetic dystrophy following nail biopsy. J Am Acad Dermatol 1987; 16: 253-256.

247. Irazuzta JE, Berde CB, Sethna NF. Laser Doppler measurements of skin blood flow before, during and after lumbar sympathetic blockade in children and young adults with reflex sympathetic dystrophy syndrome. $J$ Clin Monit 1992; 8: 16-19.

248. Jacobs MJHM, Breslau PJ, Slaaf DW, Reneman RS, Lemmens JAJ. Nomenclature of Raynaud's phenomenon: a capillary microscopic and hemorheologic study. Surgery 1987; 2: 136-145.

249. Jacobs MJHM, Slaaf DW, Lemmens HAJ, Reneman RS. The use of themorheological and microcirculatory parameters in evaluating the effect of treatment in Raynaud"s phenomenon. Vasc Surg 1987; 21: 9-16.

250. Jancso $G$, Kiraly $E$, Jancso Gabor $A$. Direct evidence for an axonal site of action of capsailcin. Naunyn-Schmiledeberg's Arch Pharmac 1980; 313: 91-94.

251. Jancso $G$, Kiraly $E$, Jancso-Gabor $A$. Pharmacologically-induced selective degeneration of chemosensitive primary afferents. Nature 1977; 270: 741-743.

252. Janig $W$. Central organization of somatosympathetic reflexes in wasoconstrictor neurones. Brain Res 1975 ; 87: 305-312.

253. Janig W, Blumberg $H_{3}$ Boas RA, Campbell JN. The reflex sympathetic dystrophy syndrome: Consensus statement and general recommendations for diagnosis and climical research. In: Bond MR, Chariton JE, Woolf $\mathrm{CJ}$, eds. Pain Research and clinical management: Proceedings of the "VIth world congress on pain, Elsevier Science Publishers BV, 1991: 373-376.

254. Janig $W$, Kollmann $W$. The involvement of the sympathetic nervous system in pair. Possible neuronal mechanisms. Arzneimittelforschung 1984; 34: 1066-1073.

255. Janig $W$. Lisney S.JW. Small diameter myelinated afferents produce vasodilatation but not plasma extravasation in rat skin. J Physiol 1989; 415:477-486. 
256. Jankovic J. Post traumatic mowement disorders: central and peripheral mechanisms. Neurology 1994; 44: 2006-2014.

257. Jazat $F$, Guilbaud $G$. The 'tonic' pain-related behaviour seen in mononeuropathic rats is modulated by morphine and naloxone. Pain 1991; 44: 97-102

258. Jessell $T M$ I Iversen $L L_{\text {, }}$ Cuello $A C$. Capsaicin-induced depletion of substance $P$ from primary sensory neurones. Brain Res 1978; 152: 183-188:

259. Jupiter JB, Seiler JG, 3rd, Zienowlcz R. Sympathetic maintained pain (causalgia) associated with a demonstrable peripheral-nerve lesion. Operative treatment. J Bone Joint Surg Am 1994; 76: 1376-1384.

260. Kajander $\mathrm{KC}$. Bennett $\mathrm{GJ}$. Onsel of a painful peripheral neuropathy in rat: a partial and differential deafferentation and spontaneousi discharge in $\mathrm{A}$ beta and $\mathrm{A}$ delta primary afferent neurons. $\mathrm{J}$ Neurophysiol 1992, 68: 734-744.

261. Kajander $\mathrm{KC}$, Wakisaka S, Bennett GJ: Spontaneous discharge originates in the dorsal root ganglion at the onset of a painful peripheral neuropathy in the rat. Neurosci Lett 1992; 138: 225-228.

262. Kaplan SS. Reflex sympathetic dystrophy of the knee. Treatment using continuous epidural anesthesia. J Bone Joint Surg Am 1989; 71: 1110-1111.

263. Karlstrom L, Dahistrom $A$. The effect of different types of axonal trauma on the synthesis and transport of amine storage granules in rat sciatic nerves. J Neurobiol $1973 ; 4: 191-200$.

264. Kawano Mi Matsuoka M, Kurokawa T, Tomita $S$, Mizuno $Y$, Ueda K. Autogenic training as an effective treatment for reflex neurovascular dystrophy: a case report. Acta Paediatr Jpn 1989; 31: 500-503.

265. Kawasaki $H_{2}$, Takasaki $K$, Saito $A$, Goto $K$ Calcitonin gene-related peptide acts as a novel vasodilator neurotransmitter in mesenteric resistance vessels of the rat. Nature $1988 ; 335: 164-167$.

266. Kawasaki $T$. Sasayama $S$, Yagi $S$, Asakawa T. Hirai T. Non-invasive assessment of the age-related changes in stiffness of major branches of the human arteries. Cardiovasc Res 1987; 21: 678-687.

267. Kaysier V, Desmeules ل, Guilbaud G. Systemic clonidine differentially modulates the abnormal reactions to mechanical and thermal stimuli in rats with peripheral mononeuropathy. Pain 1995, 60: 275-285.

268. Kayser $V_{\text {, }} \mathrm{Lee} S \mathrm{SH}_{\mathrm{b}}$ Guilbaud $\mathrm{G}$. Evidence for a peripheral component in the enhanced antinociceptive effect of a low dose of systemic morphine in rats with peripheral mononeuropathy. Neuroscience $1995 ; 64$ : $537-545$.

269. Kayser $V$, Neil $A$, Guilbaud $G$. Repeated low doses of morphine induce a rapid tolerance in arthritic rats but a potentiation of opiate analgesia in normal animals. Brain Res 1986; 383: 392-396.

270. Keen P. Harmar AJ, Spears F. Winter E. Biosynthesis, axonal transport and turnover of neuronal substance P. Ciba Found Symp 1982; 145-164.

271. Kenins P. Identification of the unmyelinated sensory nerves which evoke plasma extravasation in response to antidromic stimulation. Neurosci Lett 1981; 25: 137-141.

272. Kerouac $R$, Jacques $L_{n}$ Couture $R$. Intrathecal administration of substance $P$ enhances cutaneous plasma protein extravasation in pentobarbital anaesthetized rats. Eur J Pharmacol 1987; 133: 129-136.

273. Kesler RW, Saulsbury FT, Miller LT, Rowlingson JC. Reflex sympathetic dystrophy in children: treatment with transcutaneous electric nerve stimulation. Pediatrics 1988; 82: 728-732.

274. Khalil Z, Helme RD. Serotonin modulates substance P-induced plasma extravasation and vasodilatation in rat skin by an action through capsaicin-sensitive primary afferent nerves. Brain Res 1990; $527: 292-298$.

275. Kibler RF, Nathan PW. Relief of pain and paresthesia by nerve block distal to a lesion. J Neurol Neurosurg Psychilatry $1960 ; 23: 91-98$.

276. Klenbouck R. Uber akute Knochenatrophie bel Entzündungsprozessen an den Extremitäten (Fälschlich sogenannte Inaktivitatsatrophie der Knochen) und ihre Diagnose nach dem Röntgenbilde. Wien Med Wochenschr 1901; 51: 1345-1348.

277. Kim $H J$, Kozin $F$, Johnson $R P_{\text {i }}$ Hines $R$. Reflex sympathetic dystrophy syndrome of the knee following meniscectomy. Report of three cases. Arthritis Rheum 1979; 22: 177-181.

278. Kim JM, Arakawa $K$, VonLintel $T$. Use of the pulse-wave monitor as a measurement of diagnostic sympathetic block and of surgical sympathectomy. Anesth Analg 1975; 54: 289-296.

279. Kim SH, Chung JM. Sympathectomy alleviates mechanical allodynia in an experimental animal model for neuropathy in the rat. Neurosci Lett 1991; 134: 131-134.

280. Kim SH, Chung JM. An experimental model for peripheral neuropathy produced by segmental spinal nerve ligation in the rat. Pain 1992; 50: 355-363.

281. Kim SH, Na HS, Sheen $K_{1}$ Chung JM. Effects of sympathectomy on a rat model of peripheral neuropathy. Pain 1993: 55: 85-92.

282. King $\mathrm{JH}_{\mathrm{n}}$ Nuss $\mathrm{S}$. Reflex sympathetic dystrophy treated by electroconvulsive therapy: intractable pain, depression, and bilateral electrade ECT. Pain 1993; 55: 393-396.

283. Kingery WS, Castellote JM, Wang EE. A loose ligature-induced mononeuropathy produces hyperalgesias mediated by both the injured sciatic nerve and the adjacent saphenous nerve. Pain 1993; 55: 297-304. 


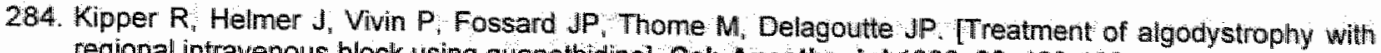
regionall intravenous block using guanethidine]. Cah Anesthesiol 1988; $36 ; 183-190$.

285. Kirseh K. Das Sudeck-Syndrom als Fernstöung (Klinik und Histologie) $Z$ Orthop 1978; 116:199-203.

286. Kitslaar PJEHM. Doppler ultrasound tests in the diagnosis of chronic aortoiliac obstruction. Maastricht: University of Limburg, The Netherlands, 1982.

287. Kleinert $H E_{1}$ Cole NM, Wayne $L_{s}$ Harvey $R_{s}$ Kutz JE, Atasoy $E$. Post-traumatic sympathetic dystrophy. Orthop Clin North Am 1973; 4: 917-927.

288. Koizumi $K$, Collier $R$, Kaufman $A$, et al: Contributions of unmyelinated afferent excitation to sympathetic reflexes. Brain Res 1970; 20: 99-106.

289. Koltzenburg $M_{1}$ Lundberg LE, Torebjork. HE. Dynamic and static components of mechanical hyperalgesia in human hairy skin [published erratum appears in Pain 1993 Jun; 53(3):363]. Pain 1992; 51: 207-219.

290. Koltzenburg $M$, Torebjork HE. Pain and hyperalgesia in acute inflammatory and chronic neuropathic conditions [letter; comment] [see comments]. Lancet 1995; 345: 1111.

291. Kool MJ, Hoeks AP, Struijker-Boudier HA, Reneman RS, Bortel van LM. Short and long-term effects of smoking on arterial wall properties in habitual smokers. J Am Coll Cardiol 1993; 22 (7): 1881-1886.

292. Kool MJ, Merode van T, Reneman RS, Hoeks APG, Struyker Boudier HA, Bortel van LM. Evaluation of reproducibility of a vessel wall movement detector system for assessment of large artery properties. Cardiovasc Res 1996; 28: 610-614.

293. Kozin F, Genant HK, Bekerman C, McCarty DJ. The reflex sympathetic dystrophy syndrome. II. Roentgenographic and scintigraphic evidence of bilaterality and of periarticular accentuation. Am J Med 1976; 60: 332-338.

294. Kozin F, McCarty D., Sims $d$, Genant $H$. The reflex sympathetic dystrophy syndrome. 1. Clinical and histological studies: evidence for bilaterality, response to corticosteroids and articular involvement. Am J Med 1976; 60: $321-331$.

295. Kozin F, Ryan LM, Carerra GF, Soin JS, Wortmann RL. The reflex sympathetic dystrophy syndrome (RSDS). III. Scintigraphic studies, further evidence for the therapeutic efficacy of systemic corticosteroids, and proposed diagnostic criteria. Am J Med 1981; 70: 23-30.

296. Kupers $R C$, Nuytten $D$, De Castro Costa $M$, Gybels $J M$. A time course analysis of the changes in spontaneous and evoked behaviaur in a rat model of neurapathic pain. Pain 1992; 50: 101-111.

297. Kurvers HAJM, Hofstra L, Hoeks APG, Van den Wildenberg FAJM, Kitslaar PJEHM, Jacobs M.HM. Sympathetic dysfunction in Sudeck's dystrophy assessed by vessel wall echo tracking. Eur $\mathrm{J}$ Clin Invest 1992; 22: A31 (Abstract).

298. Kurvers HAJM, Jacobs MJHM denervation? Clin Sci 1994; 87; 663-669.

299. Kurvers HAJM, Jacobs MJHM, Beuk RJ, et al. Reflex sympathetic dystrophy: evollution of microcirculatory disturbances in time. Pain 1995; 60: 333-340.

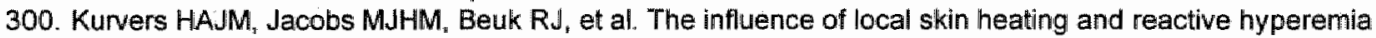
on skin blood flow abnormalities in patients with reflex sympathetic dystrophy. Eur J Cilin Invest 1995; 25 : 346-352.

301. Kurvers HAJM, Jacobs MJHM, Beuk RJ, et al. The spinal component to skin blood flow abnormalities in reflex sympathetic dystrophy. Arch Neurol 1996; 53: 58-65.

302. Kurvers HAJM, Slaaf DW, Tangelder GJ, et al. Skin blood flow abnormalities in a rat model of neuropathic pain: result of a decrease instead of an increase in efferent sympathetic nerve discharge? int $\mathrm{J}$ Microcirc : Clin Exp 1994: 14 (suppl 1): 44(Abstract).

303. Kurvers HAJM, Tangelder GJ, De Mey JGR, et al. Skin blood flow abnormalities in a rat model of neuropathic pain; result of decreased sympathetic vasoconstrictor outflow? J Auton Nerv Syst 1997 ; 63 : $19-29$.

304. Kuschner $\mathrm{SH}_{n}$ Brien WW, Johnson $\mathrm{D}$, Gellman $\mathrm{H}$. Complications associated with carpal tunnel release. Orthop Rev 1991; 20: 346-352.

305. Kvemebo K, Slagsvold CE, Stranden E, Kroese A, Larsen S. Laser Doppler flowmetry in evaluation of lower limb resting skin circulation. A study in healthy controls and atherosclerotic patients. Scand J Clin Lab Invest 1988; 48: 621-626.

306. Lagier R. Partial algodystrophy of the knee. An anatomico-radiological study of one case. J Rheumatol $1983 ; 10: 255-260$.

307. Lagier R, Chamay A. Localized Sudeck's dystrophy and distal interphalangeal osteoarthritis of a finger: anatomicoradiologic study. J Hand Surg Am 1984; 9 : 328-332.

308. Lagier $R$, Gerster JC, Favre $X$, Saudan $Y$. Post-traumatic algodystrophy localized in a metacarpal head near synovial gouty microtophi. ROFO 1984; 141: 594-597. 
309. Laird JM. Bennett Gul. An electrophysiologicall study of dorsal hom neurons in the spinal cord of rats with an experimental peripheral neuropathy. J Neurophysiol 1993; 69: 2072-2085.

310. Laird JMA, Bennet GJ. Dorsal root potentials and afferent input to the spinal cord in rats with an experimental peripheral neuropathy. Brain Res $1992 ; 584: 181-190$.

311. LaMotte $\mathrm{RH}_{\mathrm{n}}$ Shain $\mathrm{CN}$, Simone $\mathrm{DA}$, Tsai $E F$. Neurogenic hyperalgesia: psychophysical studies of underlying mechanisms. $d$ Neurophysiol 1991, 66: 190-211.

312. Langendijk PN, Zutumond WW, van Apeldoom HA, van Loenen AC, de Lange JJ, Good results of treatment of reflex sympathetic dystrophy with a $50 \%$ dimethylsulfoxide cream]. Ned Tijdschr Geneeskd 1993: 137: $500-503$.

313: Lankford LL. Thompson JE. Reflex sympathetic dystrophy, upper and lower extremity: diagnosis and management: In: AnonymousThe American Academy of Orthopaedic Surgeons. Instructional course lectures, St Louis CV Mosby, 1977: 163-178.

314. Lantsberg $L$, Goldman $M$. Lower limb sympathectomy assessed by laser Doppler blood flow and transcutaneous oxygen measurements. J Med Eng Technol 1990, 14: 182-183

315. Lasserre B. [Algoneurodystrophy, a diagnosis often unrecognized at an early stage], Rev Med Suisse Romande $1986 ; 106: 269-270$.

316. Lee $\mathrm{SH}_{3}$ Kayser $\mathrm{V}$, Desmeules d, Guilbaud $\mathrm{G}$. Differential action of morphine and various opioid agonists on thermal allodynia and hyperalgesia in mononeuropathic rats. Pailn 1994, 57: 233-240.

317. Lembeck $F$, Donnerer J. Time course of capsaicin-induced functional impairments in comparison with changes in neuronal substance P content. Naunyn-Schmiedeberg's Arch Pharmac 1981; 316: $240-243$.

318. Lembeck F, Holzer $P$. Substance $P$ as neurogenic mediator of antidromic vasodilatation and neurogenic plasma extravasation Naunyn-Schmiedberg's Arch Pharmac 1979; 310, 175-183.

319. Lenders JWM, Peters JHM; Pleters GFF, Willemsen $\mathrm{JJ}$, Thien T. Hemodynamic reactivity to sympathoadrenal stimulation in adrenalectomized women. J Clin Endocrin Metabol 1988; 67; 139-143.

320. Leriche R. De la causalgie, envisagee comme une nevrite du sympathique et de son traitement par la dentudation et l'excision des plexus nerveux peri-arteriels Presse Med 1916; 24: 178-180.

321. Levine JD, Dardick SJ, Basbaum Al, Scipio E. Contribution of the peripheral nervous system to spatially remote inflammatory responses that follow injury. J Neurosci $1985 ; 5: 1380-1386$.

322. Levine JD, Taiwo YO, Collins SD, Tam JK. Noradrenaline hyperalgesia is mediated through interaction with sympathetic postganglionic neurone terminals rather than activation of primary afferent nociceptors. Nature 1986; 323: 158-160.

323. Lewis D. Treatment of causalgia: results of intraneural injections of $60 \%$ alcohol. JAMA $1920 ; 74: 1-4$.

324. Lewis T. The blood vessells of the human skin and their responses. London: Shaw and Sons, 1927.

325. Lewis $\mathrm{T}$. Experiments relating to cutaneous hyperalgesia and its spread through somatic nerves: Clin Sil 1936; $2: 373-423$.

326. Lewis T. Pain. London: Macmillan, 1942.

327. Lightman $H I$, Pochaczevsky $R$, Aprin $H$, llowite NT. Thermography in childhood reflex sympathetic dystrophy. J Pediatr 1987; 111: 551-555.

328. Linderoth $B$, Gunasekera L. Meyerson BA. Effects of sympathectomy on skin and muscle microcirculation during dorsal column stimulation: animal studies. Neurosurgery 1991:29:874-879

329. Lindh $B$, Pelto Huikko $M_{1}$ Schalling $M$, Lundberg JM, Hokfelt-T. Substance $P$ mRNA is present in a population of CGRP-immunoreactive cholinergic postganglionic sympathetic neurons of the cat: evidence from combined in situ hybridization and immunohistochemistry. Neuroscil Lett 1989; 107: 1-5.

330. Lindvall $O$, Bjorklund $A$, Nobin $A$, Stenevi $U$. The adrenergic innervation of the rat thalamus as revealed by the glyoxylic acid fluorescence method. J Comp Neurol 1974: 154: 347-3.47.

331. Linson MA, Leffert $R_{6}$ Todd DP. The treatment of upper extremity reflex sympathetic dystrophy with prolonged continuous stellate ganglion blockade. J Hand Surg Am 1983; 8: 153-159.

332. Litwin MS. Postsympathectomy neuralgia. Arch Surg 1962; 84: 121-125.

333. Livingston WK. Pain mechanism: a physiologic interpretation of causalgia and its related states. New York: Macmillan Publishing Company, 1943, 224-230.

334. Loew M. [Sudeck"s dystrophy following arthroscopy of the knee joint--a case report]. Aktuel Traumatol 1988; 18: $157-159$

335. Loh L, Nathan PW. Painful peripheral states and sympathetic blocks. I Neuroll Neurosurg Psychiatry $1978 ; 41: 664-671$

336. Loh L, Nathan PW, Schott GD, Wilson PG. Effects of regional guanethidine infusion in certain painful states. J Neurol Neurosurg Psychiatry 1980; 43: 446-451.

337. Lombard WP. The blood pressure in the arteriolles, capillaries and small veins of the human skin. Am $\mathrm{J}$ Physlol 1912; $29: 335-362$. 
338. Loven C. Uber die Erweiterung von Arterien in Folge einer nervenerregung. Berichte uber die Verhandlungen der Koniglich sachsisichen Geselschaft der Wissenschaften zu Leipzig. Mathemathisch-physische Classe 1866; 18: 85-110.

339. Low $A$, Westerman RA. Neurogenic vasodilation in the rat hairy skin measured using a laser Doppler flowmeter. Life Sci 1989; 45: 49-57.

339. Low LC, McCruden DC "Ramsay LE. Shoulder-hand syndrome after laparoscopic sterilisation. Br Med J 1978; 2: $1059-1060$.

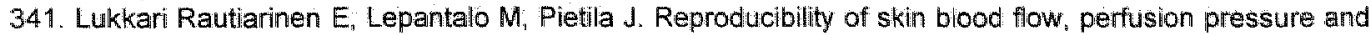
oxygen tension measurements in advanced lower limb ischaemia. Eur J Vasc Surg 1989; 3: 345-350.

342. Lundborg G, Dahlin LB. Pathophysiology of nerve compression: In: Szabo KM, ed. Nerve compression syndromes, Thorofare, N J SLACK, 1994: 15-39.

343. Lubbers DW. Theory and development of transcutaneous oxygen pressure measurement. In: Tremper $\mathrm{KK}_{\text {s }}$ Barker $S J_{\text {u }}$ eds. Advances in oxygen monitoring, Boston: Little, Brown $æ$ Company, 1987: 50-65.

344. Lynch ME. Psychological aspects of reflex sympathetic diystrophy: a review of the adult and paediatric literature. Pain 1992; 49: 337-347.

345. Mailis A, Wade J. Profile of caucasian women with possible genetic predisposition to reflex sympathetic dystrophy: a pilot study. Clin J Pain 1994; 10: 210-217.

346. Mao J Hayes RL, Price DD, Coghill RC, Lu J, Mayer DJ. Post-injury treatment with GM1 ganglioside reduces nociceptive behaviors and spinal cord metabolic activity in rats with experimental peripheral mononeuropathy. Brain Res 1992; 584: 18-27.

347. Mao J, Price DD, Coghill RC, Mayer DJ. Hayes RL. Spatial patterns of spinal cord-2-deoxyglucose metabolic activity in a rat model of painful peripheral mononeuropathy. Pain 1992; 50: 89-100.

348. Mao J, Price DD, Mayer DJ, Lu J, Hayes RL. Intrathecal MK-801 and local herve anesthesia synergistically reduce nociceptive behaviors in rats with experimental peripheral mononeuropathy. Brain Res 1992; 576 254-262.

349. Marini $F, V e c l a n i G$. [New therapeutic prospectives in reflex algodystrophy of the legs. Local intradermal calcitonin]. Chir Ital 1984; 36: 864-871.

350. Markoff M. Farole A. Reflex sympathetic dystrophy syndrome. Case report with a review of the literature. Oral Surg Oral Med Oral Pathol 1986; 61: 23-28.

351 . Mastroianni A. Sympathetic regional blocks with guanethidine diluted in 10\% dextran 40 (Rheomacrodex). Panminerva Med 1987; 29: 325-329.

352. Matsumura $H$, Sakurada T, Hara A, Sakurada S, Kisara K. Characterization of the hyperalgesic effect induced by intrathecal injection of substance $P$. Neuropharmacology $1985 ; 24: 421-426$.

353. Maurer $A H_{2}$ Holder LE, Espinola DA, Rupani HD, Wilgis EF. Three-phase radionuclide scintigraphy of the hand. Radiology 1983; 146: 761-775.

354. Maves TJ, Pechman PS, Gebhart GF, Meller ST. Possible chemicall contribution from chromic gut sutures produces disorders of pain sensation like those seen in man [published erratum appears in Pain 1993 Oct; 55(1):131-4] [see comments]. Pain 1993; 54: 57-69.

355. Mayfield FH. Causalgia. Illinois: Spring field, 1951; 3-54.

356. McLachlan $E M_{\text {, Jang }} W$, Devor $M_{n}$ Michaelis $M$. Peripheral nerve injury triggers noradrenergic sprouting within dorsal root glanglia. Nature $1993 ; 363: 543-546$

357. MoMahon SB. Mechanisms of sympathetic pain. Br Med Bulll 1991; 47: $584-600$

358. Mecklem D. Brimacombe J. EMLA cream in the treatment of causalgic pain [letter]. Anesthesiology 1994; 81: $267-268$.

359. Meller ST, Gebhart GF, Maves TJ. Neonatal capsaicin treatment prevents the development of the thermal hyperalgesia produced in a model of neuropathic pain in the rat. Pain 1992; 51: 317-321.

360. Melzack R. Clinical aspects of pain. $\| \mathrm{n}$ : Anonymous The puzzle of pain, New York: Basic Books, 1973: $60-65$.

361. Melzack R, Wall PD. Pain mechanisms: A new theory. Science $1965 ; 150: 97$ t -978.

362. Mendell LM. Physiological properties of unmyelinated fiber projections to the spinal cord. Exp Neurol 1966: 16: $316-332$.

363. Mescon $\mathrm{H}$, Hurley $\mathrm{J}$, Moretti $\mathrm{G}$. The anatomy and histochemistry of the arteriovenous anastomosis in human digital skin. J linvest Dermatol 1956; 27 : 133-144.

364. Meyer GA. Fields HL. Causalgia treated by selective large fibre stimulation of peripheral nerve. Brain 1972; 95: $163-168$.

365. Meyer RA, Raja SN, Campbell JN. Mackinnon SE, Dellon AL. Neural activity originating from a neuroma in the baboon. Brain Res 1985; 325: 255-260.

366. Miller DS $;$ DeTakats $G$. Post-traumatic dystrophy of the extremities: Sudeck's atrophy. Surg Gyn Obstet $1942 ; 75 ; 558-582$ 
367. Mitchell $5 W$. On the diseases of nerves resulting from injuries. In: Flint $A_{i}$ ed. Contributions relating to the causation and prevention of disease, and to camp diseases, New York: United states Sanitary Commission Memoirs, $1867: 412-468$.

368. Mitchell SW: injuries of nerves and their consequences. New York: Dover publications, 1877; 363-368

369. Mitchell SW, Morehouse GR, Keen WW, Gunshot wounds and injuries of nerves. Philadelphia: IB Lippencott: 1864.

370. Moesker $A_{1}$ Boersma FP, Schijgrond HW, Corturiendt W Treatment of posttraumatic sympathetic dystrophy (Sudeck's atrophy) with guanethidine and ketanserin. Pain Clinic 1985; 1: 171-176.

371. Monsivais $J^{J}$ Baker $J$, Monsivais $D$. The association of peripheral nerve compression and reflex sympathetic dystrophy. J Hand Surg Br 1993; 18:337-338.

372. Morettin LB. Wilson M. Severe reflex algodystrophy (Sudeck's atrophy) as a complication of myelography: report of two cases. Am J Roentgenol Radium Ther Nucl Med 1970; 110: 156-158.

373. Moskowitz $E_{1}$ Bishop HF, Pe H. Posthemiplegic reflex sympathetic dystrophy. JAMA 1958; 167: 836-838.

374. Muller A, Farcot JM. [Sympathetic nervous system, pain and epidural administration of morphine]. Agressologie 1991; 32: 283-286.

375. Mulvany $M \cdot$ i, Halpern $W$. Contractile properties of small arterial resistancevessels in spontaneously hypertensive and normotensive rats. Circ Res 1977, 41: 19-26.

376. Munger BL, Bennett GJ, Kajander KC. An experimental painfull peripheral neuropalthy due to nerve constriction. I. Axonal pathology in the sciatic nerve. Exp Neurol 1992; 118: 204-214.

377. Muramatsu I. Peripheral transmission in primary sensory nerves. Jpn J Pharmacol 1987; 43: 113-120.

378. Murata $K$, Nakagawai $I$, Kumeta $Y$, Kitahata $L M$, Collins JG. Intrathecal clonidine suppresses naxiously evoked activity of spinal wide dynamic range neurons in cats. Anesth Analg 1989; 69: 185-191.

379. Nagy $\mathrm{JL}_{\mathrm{n}}$ Vincent $\mathrm{SR}$, Staines WA, et al. Neurotoxic action of capsaicin on spinal substance $\mathrm{P}$ neurones. Brain Res 1980; 186: 435-444.

380. Nahin RL, Ren K, De Leon M, Ruda M. Primary sensory neurons exhibit altered gene expression in a rat model of neuropathic pain. Pain 1994; 58: 95-108.

381. Nathan PW. On the pathogenesis of causalgia in peripheral nerve injuries. Brain 1947; $70: 145$.

382. Neil $A$, Attall N, Guilbaud $G$. Effects of guanethidine on sensitization to natural stimuli and self-mutilating behaviour in rats with a peripheral neuropathy. Brain Res 1991; 565: 237-246.

383. Neil $A$, Kayser $V$, Chen $Y L$, Guilbaud $G$. Repeated low doses of morphine do not induce tolerance but increase the opioid antinociceptive effect in rats with a peripheral neuropathy. Brain Res 1990; 522 : $140-143$.

384. Nickerson $M$, Collier $B$. Drugs inhibiting adrenergic nerves and structures innervated by them. In: Goodman LS, Gilman A, eds. The pharmacological basis of therapeutics, 5th ed. New York: MacMillan, 1975: 553-554.

385. Nickeson RA, Brewer EJ, Person D. Early histological and radionuclide scan changes in children with RSD syndrome. Arthritis Rheum 1985; 28 (suppl): 72(Abstract).

386. Nielsen $H_{1}$ Pillegaard HK, Hasenkam JM, Mortensen FV, Mulvany-MJ. Heterogeneity of postjunctional alpha-adrenoceptors in isolated mesenteric resistance arteries from rats, rabbits Cardiovase Pharmacol 1991: 18: 4-10.

387. Nilsson $G E_{i}$ Tenland $T$, Obert PA. A new instrument far cantinuouis measurement of tissue blood flow by light beating spectroscopy. IEEE Trans Biomed Eng 1980; 27: 12-19.

388. Nilsson H. Adrenergic nervous control of resistance and capacitance vessels. Studies on isolated blood vessels from the rat: Acta Physioll Scand Suppl 1985; 541: 1-34.

389. Noddeland $H_{1}$. Aukland $K$, Nicolaysen $G$. Plasma colloid osmotic pressure in venous blood from the human foot in orthostasis. Acta Physioll Scand 1981: 113: 447-454:

390. Noordenbos W. Pain: problems pertaining to the transmission of nervous impulses which give rise to pain; preliminary statements. Amsterdarn: Elsevier, 1955.

391. Ochoa JL, Yarnitsky D. Mechanical hyperalgesias in neuropathic pain patients: dynamic and static subtypes. Ann Neurol 1993; 33: 465-472.

392. Ochoa لJ Y, Yarnitsky D, Marchettini P, Dotson R, Cline M. Interactions between sympathetic vasoconstrictor outflow and $C$ nociceptor-induced antidromic vasodilatation. Pain 1993; 54: 191-196.

393. Olson WL. Dystonia and reflex sympathetic dystrophy induced by ergotamine. Mov Disord 1992; 7 : 188-189.

394. Olson WLJ Perineal reflex sympathetic dystrophy treated with bilateral lumbar sympathectomy. Ann Intern Med 1990; 113: 633-634.

395. Omer GEJ. Management techniques for the painful upper extremity. Instr Course Lect 1984; 33: 513-528.

396. Orenberg EK, Pfendt EA, Wilkinson Dl. Characterization of alpha-and beta-adrenergic agonist stimulation of adenylate cyclase activity in human epidermal keratinocytes in vitro. J Invest Dermatol 1983; 80: 503-507. 
397. Otsuka M, Konishi S. Release of substance P-like immunoreactivity from isolated spinal cord of newborn rat. Nature 1976; 264: 83-84.

398. Oyen WJG, Arntz IE, Claessens RAMJ, Van der Meer JWM, Corstens FHM, Goris RJA. Rellex sympathetic dystrophy of the hand: an excessive inflammatory response ? Pain 1993, 55: 151-157.

399. Pak TJ, Martin GM, Magness JL, Kavanaugh GJ. Reflex sympathetic dystrophy. Review of 140 cases. Minn Med 1970; 53: $507-512$

400. Palecek J, Paleckova V, Dougherty PM, Cariton SM, Willis WD. Responses of spinothalamic tract cells to mechanical and thermal stimulation of skin in rats with experimental peripheral neuropathy. J Neurophysiol 1992; 67: 1562-1573.

401. Paley D, McMurtry RY. Median nerve compression by volarly displaced fragments of the distal radius. Clin Orthop Relat Res 1987; 139-147.

402. Parkinson NA, Thom SM, Hughes AD, Sever PS, Mulvany MJ, Nielsen-H. Neurally evoked responses of human isolated resistance arteries are mediated by both alpha 1 - and alpha2-adrenoceptors. $\mathrm{Br} \mathrm{J}$ Pharmacol 1992; 106: 568-573:

403. Patman RD, Thompson JE, Persson AV. Management of post-traumatic pain syndromes: report of 113 cases. Ann Surg 1973; 177; 780-787.

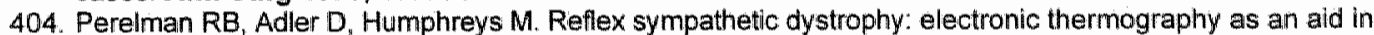
diagnosis. Orthop Rev 1987; 16: 561-566.

405. Perl E. Causalgia: sympathetically-aggravated chronic pain from damaged nerves. Pain: Clin Updates 1993; I (Issue 4): 1-4.

406. Perl ER. Causalgia and reflex sympathetic dystrophy revisited. In: Boivie $P$, Hansson $P$, Lindblom $U_{\text {, eds. }}$ Touch, temperature, and pain in health and disease: mechanisms and assessments, progress in pain research and management, Seattle: liASP Press, 1994: 231-248.

407. Perrot $S$, Attal N, Ardid D, Guilbaud $G$. Are mechanical and cold allodynia in mononeuropathic and arthritic rats relieved by systemic treatment with calcitonin or guanethidine? Pain 1993; 52: 41-47.

408. Plewes LW. Sudeck's atrophy in the hand. J Bone Joint Surg Br 1956; 38: 195-203.

409. Poehling GG, Pollock FEJ, Koman LA. Reflex sympathetic dystrophy of the knee after sensory nerve injury. Arthroscopy $1988 ; 4: 31-35$.

410. Poplawski ZJ, Wiley AM, Murray JF. Post-traumatic dystrophy of the extremities. J Bione لJoint Surg Am 1983: 65: 642-655.

411. Portwood MM, Lieberman JS, Taylor RG. Ultrasound treatment of reflex sympathetic dystrophy. Arch Phys Med Rehabill 1987; 68: 116-118.

412. Price DD, Bennet Gل, Rafil A. Psychophysical observations on patients with neuropathic pain relieved by a sympathetic block. Pain 1988; 36: 273-288.

413. Price DD, Long $\mathrm{S}$, Huitt $\mathrm{C}$. Sensory testing of pathophysiological mechanisms of pain in patients with reflex sympathetic dystrophy. Pain 1992; 49: 163-173.

414. Price DD, Mao JR, Coghill RC, et al. Regional changes in spinal cord glucose metabolism in a rat model of painful neuropathy. Brain Res 1991; 564: 314-318.

415. Pries AR. Eriksson SE, Jepsen H. Real-time oriented image analysis in microcirculatory research. Int Soc for Optical Eng 1990; 1357.

416. Priollet $P$, Fichelle $J M$, Vayssairat $M$, Cormier JM, Housset $E$. Algodystrophy after vascular surgery. Lancet $1984 ; 2: 923-924$

417. Prithvi R, Calodney A, Janisse $T$, Cannella J. Reflex Sympathetic Dystrophy. In: Jupiter, ed. Skeletal trauma, Philadelphia: W.B. Saunders Comp. 1992: 471 499.

418. Prough DS, McLeskey $C H$. Poenling GG, et al. Efficacy of oral nifedipine in the treatment of reflex sympathetic dystrophy. Anesthesiology 1985; 62: 796-799.

419. Raj P, Calodney A, Janisse T, Cannella J. Reflex Sympathetic Dystrophy. Im: Browner B, ed. Skeletal Trauma, Philadelphia: Saunders, 1992: 471-499.

420. Raja SN, Treede RD, Davis KD, Campbell JN. Systemic alpha-adrenergic blockade with phentolamine: a diagnostic test for sympathetically maintained pain. Anesthesiology 1991; 74:691-698.

421. Ramamurthy S. Electroacupuncture's role in the management of reflex sympathetic dystrophy. Tex Med 1991; $87: 82$.

422. Ranft $J$, Heidrich $H$, Peters $A$, Trampisch $H$. Laser-Doppler examinations in persons with healthy wasculature and in patients with peripheral arterial occlusive disease. Angiology 1986; 37: 818-827.

423. Raskin $\mathrm{NH}_{4}$ Levinson $\mathrm{S}$, Hoffmam PM. Pickett JB, Fields HL. Postsympathectomy neuralgia. Amelioration with diphenylhydantoin and carbamazepine. Am J Surg 1974; 128: 75-78.

424. Rasminsky M. Ectopic impulse generation in pathologic nerve fibers. In: Dyck, Thomas, Lambert, Bunge, eds. Peripheral neuropathy, London: W.B. Saunders Company, 1984: 911-918. 
425. 425.asmussen TB, Freedman H. Treatment of causalgia: An analysis of 100 cases. J Neurosurg 1946 : $165-173$

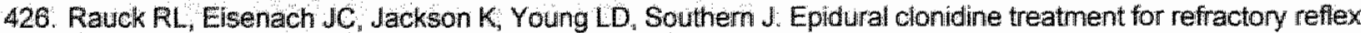
sympathetic dystrophy. Anesthesiology 1993; $79: 1163-1169$

427 Rayman $\mathrm{G}$, Hassan $\mathrm{A}$, Tooke $\mathrm{UE}$. Blood flow in the skin of the foot related to posture in diabetes mellitus. Br Med J 1986, 292:87-90.

428 Reeh $P W$, Bayer $J$ Kocher $L$, Haridwerker $H O$, Sensitization of nociceptive cutaneous merve fibers from the rat's tail by noxious mechanical stimulation Exp Brain Res 1987; 65:505-512.

429. Regan M, Foley Nolan $D$, McCarthy $G$, Coughlan RJ. Reflex sympathetic dystrophy as sociated with large cell lung carcinoma. Ann Rheum Dis $1989 ; 48: 1031$

430. Rehin J. Behandlungsergebrisse typischer Radiusfracturen. Der Chirurg 1965; $35: 206-211$.

431. Reid $J$, Wing LM, Mathias $C J$; Frankel HL. Neill $E$. The central hypotensive effect of clonidine. Studies in tetraplegic subjects. Clin Pharmacol Ther 1977; 21:375-381.

432. Reiestad F, Mcllvaine WB, Kvalheim $L$, Stokke $T$, Pettersen B. Interpleural analgesia in treatment of upper extremity reflex sympathetic dystrophy. Anesth Analg 1989; 69: 671-673.

433. Reneman RS, Van Merode T, Brands PJ, Hoeks AP. Inhomogeneities in arterial wall properties under normal and pathological conditions. J Hypertens Suppl 1992; 10: S35-9.

434. Rico Lenza H, Almoguera I, Gomez Castresana $F$, Cabranes JA, Nunez Torron M. [Osteocalcin values in reflex sympathetic dystrophy]. Med Clin Barc 1986; 86: 49-50.

435. Rieder W. Die akute Knochenatrophie. Dtsch Z Chir 1936; 248: 269-331.

436. Robaina FJ, Dominguez M, Diaz $M$, Rodriguez JL, de Vera JA. Spinal cord stimulation for relief of chronic pain in vasospastic disorders of the upper limbs. Neurosurgery 1989; 24:63-67.

437. Robaina $F J$, Rodriguez $J$, de Vera $J_{n}$ Martin MA. Transcutaneous electrical nerve stimulation and spinal cord stimulation for pain relief in reflex sympathetic dystrophy. Stereotact Funct Neurosurg 1989; 52: $53-62$.

438. Robertson D, Johnson GA, Robertson RM, Nies AS, Shand DG, Oates JA. Comparative assessment of stimuli that release netronal and adrenomedullary catecholamines in man. Circulation 1979; 59: 637-643.

439. Robertson DP, Simpson RK, Rose JE Garza JS. Video-assistied endoscopic thoracic ganglionectomy. J Neurosurg $1993 ; 79: 238-240$.

440. Rocco AG. Radiofrequency lumbar sympatholysis. The evolution of a technique for managing sympathetically maintained pain. Reg Anesth 1995; $20: 3-12$.

441. Rosen L, Ostergren J "Fagrell $B$, Stranden E. Skin microvascular circulation in the sympathetic dystrophies evaluated by videophotometric caplliaroscopy and laser Doppler fluxmetry. Eur J Clin Invest 1988; 18: 305-308.

442. Rosen L, Ostergren J, Roald OK, Stranden E, Fagrell B. Billateral involvement and the effect of sympathetic blockade on skin microcirculation in the sympathetic dystrophies. Microvasc Res 1989: 37: 289-297.

443. Rosen PS, Graham W. The shoulder-hand syndrome: Historical review with observations an 73 patients. Can Med Assoc J 1975; $77: 86-91$.

444. Rosenbloom AL. Skeletal and joint manifestations of childhood diabetes. Pediatr Clin North Am 1984; 31 : 5694889.

445. Rothberg JM, Tahmoush Ad, Oldakowski $R$. The epidemiology of causalgia among soldiers wounded in Vietnam. Mil Med 1983; 148: $347-350$.

446. Rowbotham MC, Fields HL. Topicall lidocaine reduces pain in post-herpetic neuralgia. Pain $1989 ; 38$. $297 \times 304$

447. Rowlingson JC. The sympathetic dystrophies. Int Anesthesilol Clin 1983; 21: 117-129.

448. Rudomin P. Presynaptic inhibition of muscle spindle and tendon organ afferents in the mammalian spinal cord. Trends Neurosell 1990; 13: 499-505.

449. Rumsfield JA West DP. Topical capsaicin in dermatologic and peripheral pain disorders. Ann Pharmacother $1991 ; 25: 381-387$.

450. Rumsfield JA, West DP. Topical capsaicin in dermatologic and peripheral pain disorders. DICP 1991; 25: $381-387$.

451. Rush PJ, Wilmot D, Saunders N, Gladman D, Shore A. Severe reflex neurovascular dystrophy in childhood. Arthritis Rheum 1985; 28; 952-956.

452. Santo JL, Arias LM, Barolat $G$, Schwartzman RJ, Grossman K. Bilateral cingulumotomy in the treatment of reflex sympathetic dystrophy. Pain 1990; 41: 55-59.

453. Sato $S$, Endo $T$, Koyama $K$, Fukuda $T$, Naito $H$. [A case of reflex sympathetic dystrophy following an emergency abdominal operation]. Masui 1988; 37: 1547-1551.

454. Scadding JW, Wall PD, Parry CB, Brooks DM. Clinical trial of propiranolol in post-traumatic neuralgia. Pain $1982 ; 14: 283-292$. 


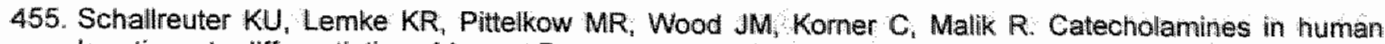
keratinocyte differentiation. J Invest Dermatol 1995; $104: 953-957$

456. Schapira D, Barron $S A$, Nahir $M_{i}$ Scharf $Y$. Reflex sympathetic dystrophy syndrome coineident with acute diabetic neuropathy. J Rheumatol 1988; 15: 120-122.

457. Schon F. Postsympathectomy pain and ehanges in sensory neuropeptides: towards an animal model. Lancet $1985: 2: 1158-1160$.

458. Schott GD. Mechanisms of causalgia and related clinical conditions. The role of the central and of the sympathetic nervous systems. Brain 1986; 109: 717-738.

459. Schuind $F$, Donkerwolcke $M_{1}$, Burny $F$. External minifixation for treatment of closed fractures of the metacarpal bones. J Orthop Trauma 1991; 5: 146-152.

460. Schwartzman RJ. Reflex sympathetic dystrophy and causalgia. Neurol Clin 1992; 10: 953-973.

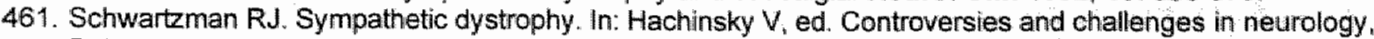
Philadelphia: F.A. Davis, 1993.

462. Schwartzman RJ. Reflex Sympathetic Dystrophy. Curr Opin Neurol Neurosurg 1993; 6: 531536.

463. Schwartzman RJ, Kerrigan J. The movement disorder of reflex sympathetic dystrophy. Neurology 1990 ; 40: $57-61$.

464. Schwartzman RJ, McLellan TL. Reflex sympathetic dystrophy. A review. Arch Neurol 1987; 44: 555-561.

465. Seldinger Sil. Catheter replacement of the needle in percutaneous arteriography. A new technique. Acta Radiol "1953; 39: 368-376.

466. Seltzer $Z$, Dubner $R$, Shir $Y$. A novel behavioral model of neuropathic pain disorders produced in rats by partial sciatic nerve injury. Pailn 1990; 43: 205-218.

467. Shea UK Perl ER. Failure of sympathetic stimulation to effect responsiveness of rabbit polymodal nociceptors. J Neurophysioll $1985 ; 54: 513-520$.

468. Shehab SA, Atkinson ME. Vasoactive intestinal polypeptide increases in areas of the dorsal horn of the spinal cord from which other neuropeptides are depleted following peripheral axotomy. Exp Brain Res $1986 ; 62: 422-430$.

469. Shibata $M$, Onkubo $T$. Takahashi $H_{\text {i }}$ Inoki $R$. Interaction of bradykinin with substance $P$ on vascular permeability and pain response: Jpn J Pharmacol 1986; 41: 427-429.

470. Shin HC, Park HJJ Raymond SA, Strichartz GR. Potentiation by capsaicin of lidocaine's tonic impulse block in isolated rat sciatic nerve. Neuroscil Lett 1994; 174: 14-16.

471. Shinya $K$, Lanzetta $M$, Conolly WB. Risk and complications in endoscopic carpal tunnel release. J Hand Surg $\mathrm{Br}$ 1995; 20: 222-227.

472. Shir $Y$, Cameron LB, Raja SN, Bourke DL. The safety of intravenous phentolamine administration in patients with neuropathic pain. Anesth Analg 1993; 76: 1008-1011.

473. Shir $Y$. Seltzer $Z$. A-fibers mediate mechanical hyperesthesia and allodynia and $C$-fibers mediate thermal hyperalgesia in a new model of causalgiform pain disorders in rats. Neurosci Lett 1990; 115: 62-67.

474. Shir $Y$, Seltzer $Z$. Effects of sympathectomy in a model of causalgiform pain produced by partial sciatic nerve injury in rats. Pain 1991; 45:309-320

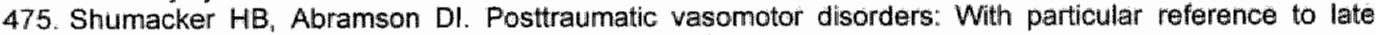
manifestations and treatment. Surg Gyn Obstet 1949; 88: 417-434.

476. Skilling SR, Harkness DH, Larson AA. Experimental peripheral neuropathy decreases the dose of substance $P$ required to increase excitatory amino acid release in the CSF of the rat spinall cord. Neurosci Lett 1992; 139: $92-96$.

477. Skilling SR, Smullin DH, Beitz AJ, Larsom AA. Extracellular amino acid concentrations in the dorsal spinal cord of freely moving rats following veratridine and nociceptive stimulation. J Neurochem 1988; 51 : 127-1.32.

478. Smith JJ, Kampine JP. Circulatory physiology: the essentials. Baltimore: Williams and Wilkins, 1980.

479. Spaendonck van KPM, Heusden van HA, The $R$, Kampen $C$, Goris RJA. Posttraumatische dystrofie en persoonlijkheidstype. In: Goris R.JA, ed. Posttraumatische dystrofie, Nymegen: Post-Academisch Onderwijs Geneeskunde Katholieke Universiteit Nijmegen, 1992: 39-43.

480. Spiegel Iل, Milowski JL. Causalgia. JIAMA 1945; 127: 9-12.

481. Spurling RG. Causalgia of the upper extremity: Treatment by dorsal sympathetic ganglionectomy. Arch Neurol 1930; 23: 784-788.

482. Stanton-Hicks M, Janig W, Hassenbusch S, Haddox JD, Boas R, Wilson P. Reflex sympathetic dystrophy; changing concepts and taxonomy. Pain 1995; 63: 127-133.

483. Stanton-Hicks M, Jänig W. Boas RA. Reflex sympathetic dystrophy. Boston: Kluwer Academic Publishers, 1990.

484. Steinbrocker $O$. The shoulder-hand syndrame. Am J Med 1947; 3; 403. 
485. Steinbrocker $O$, Argyros $T \mathrm{~T}$. The shoulder-hand syndrome in reflex dystrophy of the upper extremity. Ann Intern Med 1947:92:22.

486. Steinbrocker $O$, Argyros TG. The shoulder-hand syndrome: Present status as a diagniostic and therapeutic entity. Med Clin North Am 1958; 42: 1533-1553.

487. Stevens CW, Kajander KC, Bennett GJ, Seybold VS. Bilateral and differential changes in spinal mu, delta and kappa opioid binding in rats with a painful, unilateral neuropathy. Pain 1991, 46: 315-326.

488. Stolte $B H_{1}$ Stolte JB, Leyten JF. De pathofysiologie van het schouderhand syndroom Ned Tijdschr Geneeskd 1970; 114: 1208-1209.

489. Stricker 5 . Untersuchung uber die Gefasserwierzeln des Ischiadeus. Sitzungsber Kaiserl Akad Wiss Wien 1876; 3: 173-185.

490. Sudeck. P. Über die acute entzundliche Knochen-atrophie. Arch Klin Chir 1900, 62: 147-156.

491. Sudeck P. Die kollateralen entzundungsprozesse an den Gliedmassen (sog. akute Knochenatrophie). Arch KIIn Chir 1938; 191: 710-718.

492. Sudeck $P$. Die sogenannten akute Knochenatrophie als Entzundungsvorgang Der Chirurg $1942 ; 15$ : 449-458.

493. Sugimato $T$, Bennet GJ, Kajander KC. Transsynaptic degeneration in the superficial dorsal horn after sciatic nerve injury: Effects of a chronic constriction injury, transection and strychnine: Pain 1990; 42: 205-2॥3.

494. Sugimoto $T$, Takemura $M$, Sakai $A$, Ishimeru $M$. Rapid transneuronal destruction following peripheral nerve transection in the medullary dorsal horn is enhanced by strychnine, picrotoxin and bicuculline. Pain 1987; 30: $385-393$.

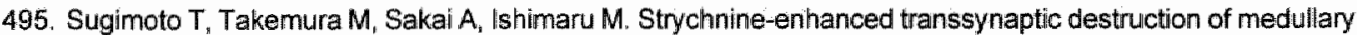
dorsal horn neurones following transection of the trigeminal nerve in adult rats including evidence of involvement of the bony environment of the transection neuroma in the peripheral mechanism. Arch Oral Biol 1987; 32: 623-629.

496. Sullivan AF, Dashwood MR, Dickenson AH. Alpha 2-adrenoceptor modulation of nociception in rat spinal cord: location, effects and interactions with morphine. Eur J Pharmacol 1987; 138: 169-177.

497. Sunderland S. Pain mechanisma in causalgia. J Neurol Neurosurg Psychiatry 1976; 39:471-480.

498. Sunderland S. The nerve lesion in the carpal tunnel syndrome: J Neurol Neurosurg Psychiatry 1976; 39 : 615-626.

499. Sunderland S. Nerves and nerve injuries. Edinburg: Churchill Livingstone, 1978.

500. Swett JE Woolf CJ. The somatotopic organization of primary afferent terminals in the superficial laminae of the dorsal horn of the rat spinal cord. J Comp Neurol 1985; 231: 66-77.

501. Swezey RL. Transient osteoporosis of the hip, foot, and knee. Arthritis Rheum 1970; 13: 858-868.

502. Szeinfeld $M$, Saucedo $R$, Pallares VS. Causalgia of vascular etiology following an abdominal injury. Anesthesiology 1982; 57: 46-47.

503. Tabira $T$, Shibasaki $H$, Kuroiwa $Y$. Refiex sympathetic dystrophy (causalgia) treatment with guanethidine. Arch Neurol $1983 ; 40: 430-432$.

504. Tahmoush AJ. Causalgia: redefinition as a clinical pain syndrome: Pain 1981; 10: 187-197.

505 . Taillan $B_{i}$ Gagnerie $F$, Gurgueghian $B$, Euller Ziegler $L$, Ziegler $G$. (Algodystrophy mimicking acute ischemia. 3 new cases (letter)]. Ann Med Interne Paris 1989; 140: 236-237.

506. Tal $M_{1}$ Bennett GJ: Extra-territorial pain in rats with a peripheral mononeuropathy: mechano-hyperalgesia and mechano-allodynia in the territory of an uninjured nenve. Pain 1994; 57: $375-382$.

507. Tanck EN, Kroin JS, MoCarthy RJ, Penn RD, Ivankovich AD. Effects of age and size on development of allodynia in a chronic pain model produced by sciatic nerve ligation in rats. Pain 1992; 51: 313-316.

508. Temesvari P. Algodystrophy and nerve lesions. Isr J Med Sci 1989, 25: 279.

509. Tenland T. Salerud EG, Nilsson GE, Oberg PA. Spatial and temporal variations in human skin blood flow. Int J Microcirc Clin Exp 1983; 2: 81-90.

510. Thomsen $M B$, Lassvik $C$, Bengtsson $M$. Changes in skin perfusion after sympathetic black with guanethidine. Int J Microcirc : Clin Exp 1988; 7: 123-130.

514. Thoumie P. Bedoiseau M. [Allergic angitits and algodystrophy. The white hand phenomenon (letter)]. Rev Rhum Mal Osteoartic 1987; 54: 79.

5 22. Tooke JE, Ostergren J, Fagrell B. Synchronous assessment of human skin microcirculation by laser Doppler flowmetry and dynamic capillaroscopy. Int J Microcirc Clin Exp 1983; 2: 277-284.

513. Torebjork HE Clinical and neurophysiological observations relating pathophysiological mechanisms in reflex sympathetic dystrophy. In: Stanton-Hicks M, Janig W, Boas RA, eds. Reflex Sympathetic Dystrophy, Boston: Kluwer academic press, 1990: 71-80.

514. Torebjork HE, Wahren IK, Wallin BG. Hallin RG. Koltzenburg M. Noradrenaline-evoked pain in neuralgia. Pailn 1995; 63: 11-20 
515. Tountas $A$ A Noguchi A. Treatment of posttraumatic reflex sympathelic dystrophy syndrome (RSDS) with intravenous blocks of a mixture of corticosteroid and lidocaine: a retrospective review of 17 consecutive cases. II Orthop Trauma 1991; 5: 412-419.

516. Treede RD, Meyer RA, Raja SN, Campbell JN. Peripheral and central mechanisms of cutaneous hyperalgesia. Prog Neurobiol 1992; 38: 397-421.

517. Tyml $K_{1}$ Ellis CG. Evaluation of the flying spot technique as a television method for measuring red cell velocity in microvessels. Int J Microcirc : Clin Exp 1982; 1: 145-155.

518. Ubbink DT. On skin microvascular reactivity in patients with lower limb ischaemia. Maastricht: PhD Thesis, University of Limburg. The Netherlands 1992.

519. Ubbink DT, Jacobs MJHM, Slaaf DW, Tangelder GJ, Reneman RS. Microvascular reactivity differences between the two legs of patients with unilateral lower limb ischaemia. Eur J Vasc Surg 1992; 6 (3): 269-275.

520. Ubbink DT, Jacobs MJHM, Slaaf DW, Tangelder GJ, Reneman RS. Capillary recruitment and pain relief on dependency in patients with severe lower limb ischaemia. Circulation 1992; 85: 223-229.

521. Ubbink DT, Jacobs MJHM, Tangelder GJ, Slaaf DW, Reneman RS. Posturally induced mierovascular constriction in patients with different stages of lower limb ischaemia: effect of local skin heating. Clin Scll $1991 ; 81: 43-49$.

522. Uematsu $S$, Hendler $\mathbb{N}$, Hungerford $D$, Long $D$, Ono $N$. Thermography and electromyography in the differential diagnosis of chronic pain syndromes and reflex sympathetic dystrophy. Electronyogr Clin Neurophysiol 1981; 21: 165-182.

523. Vaernet $K$. Two cases of shoulder-hand syndrome in meningioma affecting the premotor region. Acta Psychiatr Scand 1952; 27: 201-209.

524. Van Houdenhove B. Neura-algodystrophy: a psychiatrist's view. Clin Rheumatol 1986; 5; 399-406.

525. Van Wyngarden TM Bleyaert AL. Reflex sympathetic dystrophy inwolving the foot. J Foot Surg 1992; 31: 75-78.

526. Vanos $\mathrm{DN}$, Ramamurthy $\mathrm{S}$, Hoffman J. Intravenous regional block using ketorolac: preliminary results in the treatment of reflex sympathetic dystrophy. Anesth Analg 1992; 74: 139-141.

527. Vatner SF, Pagani M, Manders WT, Pasipoularides AD. Alpha adrenergic vasoconstriction and nitroglycerin vasodilation of large coronary arteries in the conscious dog. J Clin linvest 1980;65:5-14.

528. Veldman PH, Reynen HM, Arntz IE, Goris RJ. Signs and symptoms of reflex sympathetic dystrophy: prospective study of 829 patients. Lancet 1993,342 : 1012-1016.

529. Veldman PHJM Goris RJA. Behandeling van posttraumatische dystrofie met scavengers. Pharmaceutisch Weekbl 1994; 129: 83-85.

530. Wainapel SF. Reflex sympathetic dystrophy following traumatic myelopathy. Pain $1984 ; 18: 345-349$.

531. Wainapel SF, Freed MM. Reflex sympathetic dystrophy in quadriplegia: case report. Arch Phys Med Rehabil 1984; 65: 35-36

532. Wakisaka $\mathrm{S}_{n}$ Kajander KC, Bennet GJ. An abnormality of the cutaneous temperature and its relation to the sympathetic innerwation in rats with an experimental peripheral neuropathy. Pain 1990; supp 5.

533. Wakisaka $S_{n}$ Kajander KC. Bennett $G$ ل. Abnormal skin temperature and abnormal sympathetic vasomotor innervation in an experimental painful peripheral ineuropathy. Pain 1991" 46: $299-313$.

534. Wakisaka $S$, Kajander $K C_{\text {: }}$ Bennett GJ. Immunohistochemical analysis of the changes in neuropeptide $Y$ following peripheral nerve injury and local inflammation. In: Inoki $R$, Shigenaga $Y$. Tohyama $M$, eds. Processing and inhibition of nociceptive information, Amsterdam: Excerpta Medica, 1992: 157-160.

535. Wakisaka $S$, Shibate $M$. Takikita $S$, Yoshiya $I$, Kurisu $K$. Effects of sympathectomy on the cutaneous temperature abnormalities in rats with chronic constriction injury of the sciatic nerve. Neurosici Lett 1994; $173: 5-8$.

536. Walker AE, Nulsen F. Electrical stimulation of the upper thoracic portion of the sympathetic chain in man. Arch Neurol Psychiat 1948; 59: 559-560.

537. Walker J, Belsole R, Germain B. Shoulder-hand syndrome in patients with intracranial neoplasms. Hand 1983: 15: 347-351.

538. Wall $\mathrm{PD}$. The effect of peripheral nerve lesions and af meonatal capsaicin in the rat on primary afferent depolarization. J Physiol Lond 1982; 329: 21-35.

539. Wall $P D$, Devor $M$. The effect of peripheral nerve injury on dorsal root potentials and on transmission of afferent signals into the spinal cord. Brain Res 198: 209: 95-111.

540. Wall $P D$, Fitzgerald $M$. If substance $P$ fails to fulfil the criteria as a neurotransmilter in somatosensory afferents, what might be its function? Ciba Found Symp 1982; 249-266.

541. Wall $P D$, Gutnick $M$. Ongoing activity in peripheral nerves: the physiology and pharmacology of impulses originating from a neuroma. Exp Neurol 1974; 43: 580-593. 
542. Wall PD, Waxman S, Basbaum Al, Ongoing activity in peripheral nerve: Injury discharge Exp Neurol 1974 ; 45: $576-589$.

543. Wall $\mathrm{PD}$, Woolf $\mathrm{Cl}$. The brief and the prolonged facilitatory effects of unimyelinated afferent input on the rat spinal cord are independently irfluenced by peripheral nerve section. Neuroscience 1986; 17: 1199-1205.

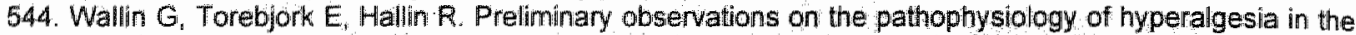
causalgic pain syndrome. In: Zotterman Y, ed. Sensony functions of the skin with special reference to man, Oxford: Pergamon Press, 1976: 489-499.

545. Wang JK, Johnson KA, Istrup DM. Sympathetic blocks for reflex sympathetic dystrophy, Pain $1985 ; 23$ 13-17.

546. Warfield CA. The sympathetic dystrophies. Hosp Pract Off 1984; 19: 52C-52J.

547. Wanwick R, Williams PL. Angiology In: Warwick R, Williams PLi, eds. Gray's anatomy, 36th ed. Edinburgh: Churchill Livingstone, 1984: 590-595.

548. Wassef: MR. Suprascapular nerve block. A new approach for the management of frozen shoulder. Anaesthesia $1992,47: 120-124$

549. Watkins JC, Evans RH. Excitatiory amino acid transmitters. Annu Rev Pharmacol Toxicol 1981; 21: $165-204$.

550. Weber ER. A rational approach for the recognition and treatment of Colles" fracture. Hand Clin 1987; 3 : 13-21.

551. Webster GF, lozzo RV, Schwartzman RJ, Tahmoush AJ Knobler RL, Jacoby RA. Reflex sympathetic dystrophy: accurrence of chronic edema and nonimmune bullous skin lesions. J Am Acad Dermatol 1993; 28: $29-32$

552. Weise WJ, Bernard DB. Reflex sympathetic dystrophy syndrome of the hand after placement of an arteriovenous graft for hemodialysis. Am J Kidney Dis 1991; 18: 406-408.

553. Werner $R$, Davidoff $G$, Jackson MD, Cremer $S$, Ventocilla $C$, Wolf $L$. Factors affecting the sensitivity and specificity of the three-phase technetium bone scan in the diagnosis of reflex sympathetic dystrophy syndrome in the upper extremity. J Hand Surg Am 1989; 14: 520-523.

554. Wesseling $\mathrm{KH}$, Wit de $\mathrm{B}$, Settels $\mathrm{JJ}$, Klawer $\mathrm{W}$. On the indirect registration of finger blood pressure after Penaz. Funkt Biol Med 1982; 1: 245-250.

555. Wettrell $G$, Hallbook $T$, Hultquist $C$. Reflex sympathetic dystrophy in two young females. Acta Paediatr Scand $1979 ; 68: 923-924$.

556. Willette RN, Hieble JP, Sauermelch CF. Sympathetic regulation of cutaneous circulation in the rat $J$ Auton Nerv Syst 1991; 32: 135-144.

557. Williams F, Birnbaum A, Wilcox $G$, Beitz A. Hybridization histochemical analysis of spinal neurons that express the alpha-2 adrenergic receptor in a rat model of peripheral mononeuropathy. Abst Soc Neurosci 1991: 17: 1370(Abstract).

558. Willis WD, Coggeshall RE. Sensory mechanisms of the spinal cord. New York: Plenum, 1991;

559. Winer $N$, Carter $C$. Effect of cold pressor stimulation on plasma norepinephrine, dopamine b-hydroxylase, and renin activity. Life Sci 1977; 20: $887-894$.

560. Wirth FP, Jr., Rutherford RB. A civlian experience with causalgia. Arch Surg 1970; 100: 633-638.

561. Wollersheim $H_{1}$ Reyenga $J$, Thien $T$. Laser Doppler Velocimetry of fingertips during heat provocation in normals and in patients with Raynaud's phenomenon. Scand J Clin Lab Invest 1988; 48: 91-95.

562. Xie YK, Xiao WH. Electrophysiological evidence for hyperalgesia in the peripheral neuropathy. Sci China 1990; 33: 663-672.

563. Yaksh TL. Pharmacology of spinal adrenergic systems which modulate spinal nociceptive processing. Pharmacol Blochem Behav 1985; 22: 845-858.

564. Yaksh TL, Noueihed R. The physiology and pharmacology of spinal opiates. Annu Rev Pharmacol Toxicol 1985; 25: 433-462.

565. Yaksli TL, Pogrel JW, Lee YW, Chaplan SR. Reversal of nerve ligation-induced allodynia by spinal alpha-2 adrenoceptor agonists. J Pharmacol Exp Ther 1995; 272: $207-214$.

566. Yamamoto $T$. Shimoyama $N$, Mizuguchi $T$. Role of the injury discharge in the development thermal hyperestesia after sciatic nerve constriction. Amesthesiology 1993; 79: 993-1002.

567. Yamamoto T, Yaksh TL. Spinal pharmacology of thermal hyperesthesia induced by constriction injury of sciatic nerve. Excitatory amino acid antagonists. Pain 1992; 49: 121-128.

568. Yokata T, Furukawa T, Tsukagoshi H. Motor paresis improved by sympathetic block: a form of reflex sympathetic dystrophy? Arch Neuroll 1989; 46: 683-687. 


\section{KORTE SAMENVATTING}

Reflex sympathische dystrofie (RSD), ook wel genoemd Sudeckse dystrofie of post-traumatische dystrofie, is een pijnsyndroom dat gewoonlijk optreedt in een extremiteit en dat meestal het gevolg is van een trauma of operatie. Het syndroom wordt gekarakteriseerd door een divers aantal klinische verschijnselen en symptomen (sensibel, autonoom (sympathisch), motorisch en trofisch) welke varieren in aanwezigheid en emst, niet alleen tussen patienten maar ook gedurende het beloop van de aandoening. RSD is een belangrijk klinisch probleem. De geschatte jaarlijkse incidentie in Nederland bedraagt 8000 . Uniform geaccepteerde diagnostische criteria zijn pas vrij recent ontwikkeld. Daarnaast bestaat nog steeds weinig duidelijkheid omtrent de exacte pathogenese. Dientengevolge zijn de bestaande behandelingen weinig tot niet gestoeld op rationele basis. Het is dan ook niet verwonderlijk dat de resultaten van de diverse behandelingsmethoden over het algemeen niet bevredigend zijn. Ondanks het feit dat bij een aanzienlijk deel van de patienten het naturlijk beloop resulteert in vrijwel volledig herstel, worden bij een deel van de patienten de kلachten chronisch. De pijn kan uitermate ernstige vormen aanmemen en leiden tot volledige invaluditeit, zeker indien het beeld gepaard gaat met ernstige motorische en/of trofische stoornissen.

De betrokkenheid van het zenuwstelsel bij RSD moge blijken uit het feit dat het beeld wordt gekarakteriseerd door een trias van sensibele, autonome en motorische stoomissen, hetgeen duidt op dysfunctioneren van de betreffende 3 typen zenuwvezels. Het is vooralsnog onduidelijk wat de exacte oorzaak is van deze veranderde neurofysiologische eigenschappen. Sensibele dysfunctie uit zich klinisch met name in de vorm van stimulus onafhankelijke alsook stimulus-geinduceerde pijn. Deze klinische fenomenen duiden op, respectievelijk, spontane ontladingen en toegenomen prikkell baarheid van sensibele zenuwen. Autonome dysfunctie wordt gekarakteriseerd door stoornissen in de huiddoorbloeding en zweetproduktie. Motorische dysfunktie uit zich in de vorm van funktiebeperking, krachtsverlies en onwillekeurige bewegingen.

Er bestaan verschillende hypotheses omtrent de oorzaak van de toename in gevoeligheid van sensibele zenuwen. Vast staat dat bij RSD patienten sprake is van een abnormale interactie tussen het sensibele en het sympathische zenuwstelsel: omstandigheden welke de sympathicus activiteit doen toenemen (koude, stress) doen vaak de ernst van de pijnklachten toenemen. Daarnaast veroorzaken zowel intracutame injectie van catecholamines alsook elektrische stimulatie van sympathische efferente zenuwen bij RSD patienten pijn, terwijl dit bij gezonde vrijwilligers niet het geval is. Het belangrijkste argument dat pleit voor een abnormale interactie is de klinische bevinding dat onderbreken van efferente sympathische zenuwactiviteit (middels chirurgische of chemische sympathectomie) de pijnklachten bij een deel van de RSD patienten doet afnemen. Op basis van laatstgenoemde bevinding wordt vaak gehypothetiseend dat ten gevolge van een overmaat aan sensibele impulsen richting CZS reflexmatig de hoeveelheid sympathische impulsen vanuit het $\mathrm{CZS}$ richting de angedane extremiteit toeneemt. Laatstgenoemd fenomeen zou op zijn beurt weer excitatie van sensibele zenuwvezels teweeg brengen. Een tweede mechanisme dat in verband wordt gebracht met de toename in gevoeligheid van sensibele zenuwen is neurogene inflammatie. Uit eerdere studies is gebleken dat (elektrische, chemische of mechanische) midaxonale excitatie van sensibele zenuwrezels leidt tot omkering van prikkelgeleiding (van midaxonaal naar perifeer toe, antidromale stimulatie). Ten gevolge hiervan komen ter plaatse van de distale uiteinden van sensibele zenuwvezels neuropeptides vrij. Deze neuropeptides brengen multipele effecten teweeg: onder andere excitatie van omliggende sensibele zenuwvezels, vasodilatatie en toename van de capillaire permeabiliteit. Deze effecten zouden een aantal klinische fenomenen kumnen verklaren: respectievelijk pijn, warmte/roodheid van de huid en oedeem.

Sinds 1988 bestaat er een dierexperimenteel model waarin de klinische verschijnselen en symptomen van RSD patienten kunnen worden teweeggebracht. Hiertoe wordt bij de rat een viertal ligaturen losjes rondom de nervus ischiadicus aangebracht, hetgeen een milde compressie veroorzaakt (chronic constriction injury (CCI). Binnen enkele dagen na deze procedure ontstaan sensibele (spontane en stimulus-geinduceerde pijn), autonome (abnormale huidtemperatuur), motorische (afwijkend looppatroon) en trofische (abnormale klauwgroei) stoornissen.

Het is van belang om te weten dat, zowel bij de mens als bij de rat, sympathische en sensibele zenuwvezels betrokken zijn bij de regulatie van de vaatwandtomus. Het bepalen van diverse 
vasculaire/hemodynamische parameters, zoals huiddoorbloeding, distensibiliteit van de arteria brachialis en (in-vitro) reactiviteit van arteriele weerstandsvaten verschaft dus een indirecte maat voor de aktiviteit/funktie van beide typen zemurvezels alsmede voor de gevoeligheid van de door betreffendle zenuwvezels geinnerveerde weefsels. In het kader van dit proefschrift zijn deze vasculaire/hemodynamische parameters dan ook bestudeerd bij RSD patienten alsmede bij ratten met partieel perifeer zenuwletsel (CCI ratten).

De bevindingen in dit proefschrift (zie fig. 14.1) suggereren dat de klinische verschijnselen en symptomen van RSD het gevolg zijn van partieel zenuwletsel. Betreffend letsel kan gelokaliseerd zijn zowel op het niveau van gemengde perifere zenuwen (compressie neuropathie, bijv. CTS) alsook ter plaatse van de perifere zenuwuiteinden (weke delen letsel, bijw. crush-injury). Meer specifiek werd in dit proefschrift aangetoond dat sympathische dysfunktie distaal van het trauma bestaat uit een toegenomen gevoeligheid voor catecholamines secundair aan partiele sympathische denervatie. Daarnaast werd aangetoond dat, in geval van partieel zenuwletsel, excitatie van zowel sympathische alsook sensibele zenuwvezels leidt tot neurogene inflammatie, gemedieerd via afgifte wan neuropeptides ter plaatse wan de perifere uiteinden van de geexciteerde zenuwvezels.

\section{Sympathische dysfunctie}

Alhoewel het algemeen is geaccepteerd dat dysfunctie van het sympathisch zenuwstelsel bijdraagt aan de klinische symptomatologie van RSD, was het exacte karakter hiervan vooralsnog onduidelijk. Tot op heden werd meestal verondersteld dat sympathische dysfunctie bij RSD patienten bestaat uit een toename van de hoeveelheid sympathische impulsen vanuit het CZS richting de aangedane extremiteit. Volgens deze theorie induceert beschadiging van gemengde perifere zenwwen en/of perifere uiteinden van sensibele zenuwen een toename van het aantal sensibele impulsen richting CZS. Deze toegenomen hoeveelheid sensibele impulsen zou reflexmatig de hoeveelheid sympathische impulsen vanuit het CZS richting de aangedane extremiteit doen toenemen (somato-sympathische reflex).

\section{(Partiële) sympathische denenvatie}

Diverse bevindingen in dit proefschrift duiden er op dat, zowel bij RSD patienten alsook bij CCI ratten, distaal van het trauma geen sprake is van een toename van de hoeveelheid efferente sympathische zenuwimpulsen maar van partiêle sympathische denervatie. Deze partiële sympathische denervatie kan op verschillende manieren tot stand zijn gekomen. Allereerst zou dit fenomeen het resultal kumnen zijn van degeneratie van sympathische zenuwvezels ter plaatse van het trauma. Ten tweede is in eerdere studies aangetoond dat diverse vormen van zenuwbeschadiging (zowel partieel alsook volledig) verminderde synthese van noradrenaline doot sympathische neuronen kunnen induceren. Onze bevindingen in hoofdstuk 9 suggereren dat proximaal van het trauma, in tegenstelling tot de bevindingen distaal van het trauma, sprake is van een toename van de hoeveelheid efferente sympathische zenuwimpulsen in alle 3 de stadia van RSD. Deze discrepantie tussen beide lokalisaties pleit ten faveure van de hypothese dat de partielle denervatie distaal van het trauma het gevolg is van degeneratie van sympathische zenuwvezels ter plaatse van het trauma.

\section{Supersensitiviteit voor catecholamines}

Diverse bevindingen in dit proefschrift duiden er op dat, zowel bij RSD patienten alsook bij CCI ratten, distaal van het trauma sprake is van hypersensitiviteit voor catecholamines. Tevens bleek bij CCI ratten dat de betreffende hypersensitiviteit gemedieerd wordt via een tweetal verschillende mechanismen. Allereerst bleek de pre-synaptische heropname van catecholamines gestoord hetgeen waarschijnlijk berust op de eerder gememoreerde degeneratie van sympathische zenuwvezels. Tevens blijkt er sprake te zijn van post-junctionele ( $\alpha_{1}$-gemedieerde) supersensitiviteit. Laatstgenoemd fenomeen kan het gevolg zijn van een toename van het aantal en/of van de gevoeligheid van $\alpha_{1}$-adrenoceptoren. Daar in eerdere studies is aangetoond dat sympathische denervatie hypersensitiviteit voor catecholamines induceert is het waarschijnlijk dat bij RSD patienten deze fenomenen causaal gerelateerd zijn. 


\section{Neurogene inflammatie}

De bevindingen in dit proefschrift suggereren dat bij CCI ratten, zowel in een vroeg alsook in een later stadium na aanbrengen van het zenuwletsel, vasodilatatie wordt teweeggebracht middels afgifte van neuropeptides ter plaatse van de distale uiteinden van sensibele en sympathische zenuwvezels. Waarschijnlijk wordt dit wrijkomen van neuropeptides bij CCI ratten geinitieerd door mechanische (constrictie) en/of chemische (componenten in cat gut, inflammatoire reactie teweeggebracht door operatieve procedure) excitatie van sensibele/sympathische zenuwvezels ter plaatse wan het trauma. Bij RSD patienten is het woorstelbaar dat neurogene inflammatie wordt geinitieerd door mechanische excitatie (direct gevolg van het trauma) danwel door chemische excitatie (inflammatoire reactie volgend op weefselbeschadiging) van sensibele/sympathische zenuwwezels ter plaatse van het trauma.

\section{Contralaterale effecten}

In dit proefschrift werd aangetoond dat zowel bij RSD patienten alsook bij CCI ratten enkele van voomoemdle effecten tevens aanwezig zijn in de contralaterale extremiteit. Bij stadium I RSD patienten bleek de huiddoorbloeding toegenomen aan de klinisch niet aangedane zijde. Onze bevindingen suggereren tevens dat de betreffende toename het gevolg is van een verminderde efferente sympathische zenuwactiviteit vanuit het CZS richting de klinisch niet-aangedane extremiteit. Tevens werd in dit proefschrift aangetoond dat bij CCI ratten de huiddoorbloeding is toegenomen aan de niet geligeerde zijde. Deze toename bleek het gevolg te zijn van 1) verminderde efferente sympathische zenuwactiviteit vanuit het CZS richting de niet-geligeerde extremiteit en 2) antidromalle impulsaktiviteit in sensibele zenuwvezels in de niet-geligeerde nervus ischiadicus.

\section{Niet-vasculaire effecten}

Sympathicus dysfunktie uit zich bij RSD patienten niet alleen in de vorm van veranderingen in huiddoorbloeding: ook abnormale zweetproduktie en trofische veranderingen van de huid zijn uitingen van dysfunctioneren van het sympathische zenuwstelsel. Bovendien is uit diverse eerdere studies gebleken dat pijnklachten bij een deel van de RSD patienten sympathico-gemedieerd zijn. Het is zeer wel voorstelbaar dat de sympathicus dysfunctie voor wat betreft deze niet vasculaire structuren eveneens bestaat uit hypersensitiviteit voor catecholamines secundair aan partiële autonome denervatie. Een dergelijk bifasisch patroon zou ook verklaren waarom in een vroeg stadium na het trauma, wanneer de hypersensitiviteit zich nog niet heeft ontwikkeld, er vaak nog geen sprake is van hyperhydrosis, trofische stoornissen van de huid en sympathico-gemedieerde pijnklachten terwijl dit bij dezelfde patienten in latere stadia well het geval kan zijn.

In eerdere studies is aangetoond dat neuropeptides niet alleen vasodilatatie teweegbrengen maar ook enkele andere effecten zoals excitatie van sensibele zenuwvezels en toename van de capillaíre permeabiliteit. Het is dan ook zeer waarschijnlijk dat, bij RSD patienten, de twee laatstgenoemde fenomenen een rol van belang spelen bij, respectievelijk, pijnklachten en oedeemvorming. Daarnaast is aangetoond dat bij neurogene inflammatie uitbreiding van vasodilatatie en hyperalgesie buiten het oorspronkelijke gebied plaats vindt. Laatstgenoemd fenomeen zou de klinische observatie kunnen verklaren dat de pijnklachten van RSD patienten op langere termijn zich nogal eens uitbreiden buiten het gebied waar het initierend trauma heeft plaatsgevonden. In lijn met deze bevindingen is bij CCI ratten aangetoond dat de pijnklachten zich uitbreiden buiten het verzorgingsgebied van de nervus ischiadicus.

De bevindingen aan de niet-aangedane zijde zouden een verkllaring kunnen vormen voor het, zowel bij RSD patienten alsook bij CCI ratten, geobserveerde spiegel-fenomeen: het ontstaan van pijnklachten (meestal in mindere mate) aan de initieel niet-aangedane zijde. Daarnaast zouden de afwijkingen aan de initieel niet-aangedane zijde een verklaring kunnen vormen voor de klinische aanname dat RSD patienten met een unilaterale aandoening gepredisponeerd lijken tot het ontwikkelen van RSD in de contralaterale extremiteit.

\section{Interaktie sympathisch-sensibel zenuwstelsel}

In dit proefschrift is aangetoond dat veranderingen in huiddoorbloeding teweeg worden gebracht door 
enerzijds hypersensituviteit voor catecholamines secundair aan partiële sympathische denervatie en anderzijds antidromale activiteit in sensibele zenuwvezels. Beide mechanismen bewerkstelligen een tegenovergesteld effect hetgeen wellicht een verklaring vormt voor de, bij RSD patienten frequent geobserveerde, korte-termijn fluctuaties in huiddoorbloeding. In geval van hoge catecholamine-spiegels in het bloed (koude, stress) zal het hypersensitiviteits-effect overheersen terwijl in geval wan mechanische excitatie yan sensibele zenuwvezels (oefenen aangedane extremiteit) de antidromale activiteit zal owerheersen:

Bij een deel van de RSD ontwikkelt zich een abnormale gevoeligheid voor catecholamines. Deze toename in gevoeligheid zou deels verklaard kunnen worden door het optreden van hypersensitiviteit secundair aan (partiele) autonome denervatie. Een tweede factor welke een rol zou kunnen spelen in het ontwikkelen van deze abnormale gevoeligheid voor catecholamines betreft het vrijkomen van neuropeptides ten gevolge van antidromale activiteit in sensibele zenuwvezels. Deze neuropeptides doen namelijk de microvasculaire permeabiliteit toenemen. Een direct gevolg hiervan zou kunnen zijn dat de bloed-zenuw barriere welke normaal gesproken niet doorlaatbaar is voor catecholamines, dit ten tijde van het bestaan van neurogene inflammatie well zou kunnen worden.

\section{Klinische relevantie}

De in de thesis beschreven studies verschaffen meer inzicht in de pathofy siologische mechanismen welke aan $\mathbb{R S D}$ ten grondslag liggen. Mogelijk bieden deze nieuwe inzichten ingangen voor andere vormen van therapeutische interventies.

Tot op heden zijn therapeutische interventies bij RSD patienten steeds gericht geweest op het aitschakellen van de vermeende toename in efferente sympathische zenuwimpulsen. Hiertoe werd een chirurgische of chemische destructie van de paravertebrale sympathische grensstreng uitgevoerd. Positieve effecten (i.e., toename huiddoorbloeding/afname pijnklachten) van deze procedures werden over het algemeen slechts gezien bij een deel van de patienten en waren daamaast meestal van tijdelijke aard. Waarschijnlijk zijn de geobserveerde positieve effecten het gevolg van het feit dat bij RSD patienten sprake is van partiële denervatie; een vervolgens uitgevoerde chemische of chirurgische sympathectomie zal de efferente sympathische zenuwactiviteit nog verder reduceren. Echter, op langere termijn zal een dergelijke therapeutische interventie de mate van hypersensitiviteit voor catecholamines verder doen toenemen. Laatstgenoemd mechanisme vormt waarschijnlijk de verklaring voor het tijdelijke effect van sympathectomie. In dit proefschrift wordt aangetoond dat sympathicus dysfunctie bij RSD patienten bestaat uit hypersensitiviteit voor catecholamines secundair aan partiële sympathische denervatie. $\mathrm{Op}$ basis van deze bevindingen lijkt het voor de hand liggend dat dient te worden uitgegaan van een andere behandelingsoptiek: blokkade van hypersensitieve adrenoceptoren middels $\alpha$ adrenoceptor blokkers. Voordelen van deze therapeutische interventie zijn onder andere dat hierdoor de mate van hypersensitiviteit woor catecholamines niet verder toeneemt en dat bij patienten bij wie ten gevolge van het trauma reeds sprake is van (sub) totale sympathische denervatie blokkade van adrenoceptonen zinvol kan zijn terwijl een chirugische/chemische sympathectomie de mate van efferente sympathische zemuwactiviteit niet verder kan doen afnemen. In dit proefschrift werd tevens aangetoond dat er sprake is van een selectieve hypersensitiviteit voor $\alpha_{1}$-adrenoceptoren. Blokkade van adrenoceptoren kan dus selectief worden toegepast waardoor hoger (en waarschijnijik effectiever) kan worden gedoseerd zonder de gebruikelijke bijwerkingen (hypotensie e.d.).

Een tweede behandelingsoptiek welke, voortbouwend op de gegevens uit dit proefschrift, zinvol zou kunnen zijn bij RSD patienten is gebaseerd op reductie van de mate van neurogene inflammatie. Dit zou op diverse manieren kunnen worden bewerkstelligd. Allereerst zou het axoplasmatisch transport văn netropeptides kunnen worden geblokkeerd middels perineurale en/of epidermale applicatie van capsaicine. Daarnaast zouden specifieke neuropeptide-receptor blokkers kunnen worden toegepast.

Uitgaande van het concept dat RSD het gevolg is van partieel zemuwletsel, zou gebruikmaken van hyperosmolaire infusievloeistoffen (zoals bijvoorbeeld mannitol 10\%), ter bestrijding van endoen perineturaal oedeem, wellicht zinvol kunnen zijn. Tevens is het voorstelbaar dat in geval van mechanische en/of chemische excitatie van een gemengde perifere zenuw chirurgische vrijleggen en/of transpositioneren van de aangedane zenuw therapeutische waarde zou kunnen hebben. 


\section{DANKWOORD}

Bij het lezen van een dankwoord in andermans proefschrift had ik altijd de idee van het ene cliché in het andere te vervallen. Pas als je zelf de rit bijna voltooid hebt realiseer je je dat het voltooien van een proefschrift in je eentje inderdaad absoluut onmogelijk is. Het doet mij dan ook bijzonder genoegen nu over te kunnen gaan tot het bedanken van allen zonder wie dit boekwerk niet tot stand zou zijn gekomen.

Prof Dr PJEHM Kitslaar, beste Peter, pas toen Michael naar het Amsterdamse vertrok ben jij bij mijn promotie-onderzoek betrokken geraakt. Alhoewel jouw voornaamste aandachtsgebied de macrocirculatie is, heb je toch op de voor jouw typerende conscientieuze wijze de begeleiding van mijn microcirculatoir georienteerd proefschrift op je genomen. Sterker nog, na enige tijd begon ik zelfs de idee te krijgen dat je ons gedelibereer over kleine bloedvaten interessant begon te vinden. Op diverse manieren heb je er zorg voor gedragen dat mijn onderzoek gecontinueerd kon worden. Je creerde de juiste randvoorwaarden en gaf me tegelijkertijd de vrijheid om het onderzoek de kant op te laten gaan welke ik zinvol achtte. Jouw meest karakteristieke eigenschap is dat je graag zoals je het zelf noemt "de advocaat van de duivel speelt". Deze eigenschap, welke op schijnbaar willekeurige momenten de kop op steekt, heeft mijn bloeddruk nogal eens tot ongekende hoogten doen stijgen, met name tijdens het bespreken van artikelen. Pas later realiseerde ik me dat deze eigenschap het "slachtoffer" dwingt tot kritisch nadenken en dientengevolge nogal eens zorg draagt voor een beter (wetenschappelijk) eindresultaat. Sinds enige tijd hebben wij gezamenlijk het microcirculatoir onderzoeksapparaat losgelaten op meer vaatchirurgisch georienteerde onderwerpen, wederom met veel genoegen mijnerzijds. Ik hoop dat onze samenwerking nog lang moge duren.

Prof Dr MJHM Jacobs, beste Michael, jij bent degene die mij op een cruciaal beslismoment de kans heeft geboden om het onderzoekspad in te slaan. Ik wist op dat moment niet dat ik terecht zou komen bij iemand die met ongekende voortvarendheid en souplesse een bliksemcarriere zou maken. Ook na je vertrek naar Amsterdam zijn we contact blijven houden, ik hoop dat dit zo zal blijven. Jij bent de laatste microcirculant voor mij die vanuit het microcirculatoire onderzoek in de opleiding algemene heelkunde terecht is gekomen. Als zodanig heb je voor mij het goede voorbeeld gezet. llk hoop dat ik in het verdere verloop van mijn chirurgische loopbaan ook in staat zal zijn om jouw voorbeeldrol bij te benen.

Prof RS Reneman, beste Rob, als ik ten behoeve van wetenschappelijke besprekingen jouw kamer binnentrad was het alsof ik oog in oog stond met de reincarnatie van Weir Mitchell (zie kaft proefschrift). De door jou ten toon gespreide wetenschappelijke diepgang en inzicht in het ziektebeeld deden dit vermoeden alleen maar verder toenemen. Het verbaasde mij hoe jij, op de voor jou typerende aimabele wijze, tijdens wetenschappelijke besprekingen binnen zeer korte tijd de vinger op de zere plek wist te leggen, zelfs indien het zoals in mijn geval materie betrof welke nieuw was binnen de onderzoekswereld van het CARIM. Jij hebt je in het verleden diverse malen uitgesproken als voorstander van serendipiteit binnen het wetenschappelijk onderzoek. Jouw voortvarende en voortdurende ondersteuning van mijn onderzoek heeft getoond dat dit niet alleen maar loze woorden zijn. Een aardige bijkomstigheid is dat jij, van oorsprong anesthesist, nu via dit zijpad weer indirect te maken hebt gekregen met de problematiek van de pijnbestrijding. 
Dr DW Slaaf; beste Dick, jouw haast mathematische aanpak heeft er zorg voor gedragen dat er enige structuur werd gebracht in mijn eerste schreden op het wetenschappelijke pad. Jouw voor de basale onderzoekswereld ongekend directe en kritische aanpak vergt enige gewenning, maar blijkt uiteindelijk zeer werkzaam. De voortvarendheid en snelheid waarmee jij manuscripten en onderzoeksideeen corrigeert is memorabel. Met name op methodologisch vlak heb jij een belangrijke bijdrage geleverd. Daarnaast was het prettig om te weten dat op jouw kamer altijd even stoom afgeblazen kon worden.

Dr FAJM van den Wildenberg, beste Frans, alhoewel jij aanvankelijk alleen betrokken was bij het ronselen van RSD patienten ten behoeve van microcirculatoir onderzoek, is jouw rol gaandeweg steeds groter geworden. Jouw inzet en betrokkenheid rechtvaardigden zeker een co-promotor schap. Helaas maakte de door de universiteit opgelegde limitering van het aantal co-promotoren een en ander onmogelijk. Het doet me deugd dat het RSD onderzoek onder jouw supervisie gecontinueerd wordt. Ik ben er van overtuigd dat de ESES-trial onder jouw bezielende begeleiding en door de inzet van Marius een succes zal worden.

Dr JGR de Mey, beste Jo, onder jouw supervisie is hoofdstuk 13 van dit proefschrift uitgevoerd, hetwelke ik nog steeds als de kroon op mijn werk beschouw. Jij beschikt over zeer diepgaande kennis van vaatwandreaktiviteit in zijn algemeenheid en het het sympathische zenuwstelsel in het bijzonder. Het was altijd een bijzonder genoegen om het lab van Jo\&Co binnen te vallen en met jou over diverse aspecten van het onderzoek van gedachten te mogen wisselen. De praktische uitvoering van de experimenten zoals beschreven in hoofdstuk 13 was in handen van Frank Stassen. Beste Frank, als twee Zittesje sjnaake samen gaan werken, dan kan dit niet anders dan goed uitpakken. Als we de volgende keer samen gaan skieen doe ik wel een sjaal om. Tevens dank aan Gregorio Fazzi en Dorette van Ingen Schenau voor assistentie bij de experimenten.

Prof GJ Tangelder, beste Geert-Jan, jij was de eerste bij wie ik te rade ben gegaan toen $\mathrm{ik}$ naast het humane onderzoek dierexperimentele studies wilde gaan uitvoeren. Het feit dat jij volledig openstond voor nieuwe ideeen heeft er zeker toe bijgedragen dat deze dierexperimentele tak een volwaardig deel van mijn proefschrift is gaan uitmaken. Ook tijdens de schrijffase bleken jouw correcties, in de vorm van weelderige groene/rode hanepoten, van onschatbare waarde. Helaas heb jij ook het Maastrichtse voor het Amsterdamse verruild. Je fillosofische inslag en onophoudelijke stroom van ideeèn zal ik missen.

Dr L Hofstra, beste Leo, jij was betrokken bij de directe uitvoering van de experimenten ten behoeve van hoofdstuk 9. Jouw indirecte bijdrage aan mijn proefschrift is echter veel groter dan dit ene hoofdstuk. Onze onderzoekslaboratoria grensden aan elkaar. De tussenliggende deur heeft letterlijk en figuurlijk altijd open gestaan. Een betere buurman en maat had ik mij niet kunnen wensen. Jouw schijnbaar onuitputtelijk optimisme werkt bijzonder aanstekelijk. Met veel genoegen denk ik onder andere terug aan gezamenlijke ski-congressen: boven op de piste, genietend van perfecte sneeuw en zon, stonden we te pochen hoe prima we onze zaakjes wel niet voor elkaar hadden. Aan alles was gedacht, zelfs aan ski-brillen. Tot we tot de ontdekking kwamen dat we één ding waren vergeten: de dia's voor onze gezamenlijke presentatie! Ook dit probleem bleek gelukkig weer oplosbaar. Onze practical jokes waren voor buitenstaanders waarschijnlijk erg vermoeiend maar hielden in ieder geval leven in de (onderzoeks-) brouwerij: je hebt er nog een paar tegoed. Graag had ik onze kameraadschap en directe samenwerking gecontinueerd gezien in de opleiding Heelkunde. Helaas heeft dit niet 
het geval mogen zijn hetgeen mij bijzonder spijt. Ik was dan ook bijzonder vergenoegd dat je een andere opleidingsplaats hebt weten te bemachtigen in het AZM. Ik ben er van overtuigd dat men ook binnen de cardiologie onder de indruk zal zijn van jouw bijzonder hoogstaande (vasculaire) kwaliteiten. Het doet mij bijzonder deugd dat jij, net terug uit de VS, als paranymf aanwezig zult zijn: je had niet mogen ontbreken.

Drs Roland Beuk, beste Roland, niet alleen bakkerszonen uit Weert, maar ook muzikanten-zonen uit Wassenaar zijn van harte aan te bevelen als student-assistent. Aanvankelijk verontruste jouw Wassenaarse afkomst mij enigszins; het laatste waar ik behoefte aan had was een werkschuw, verwend kereltje. Gelukkig kwam ik er al snel achter dat je niet de doorsnee Wassenaar representeerde: zo bleek je in het bezit van slechts eén (ribfluwelen) broek en reed je rond in een gare BMW met dobbelstenen aan de spiegel en een groot hoefijzer op de radiateur. Je hebt niet alleen geweldig veel werk voor me verzet maar bleek ook nog eens over een zeer bijzonder (of bizar?) gevoel voor (beukensiaanse) humor te beschikken. Met veel plezier denk ik onder andere terug aan het door ons bezochte congres in Portugal. Tijdens een vrije middag besloten we met een catamaran het ruime sop te kiezen. Natuurlijk hielden wij ons niet aan het uitdrukkelijke verzoek van de bootverhuurder om niet te ver uit de kust te gaan, tot we een met tapijten ventende Marokkaan op een kameel tegenkwamen. Er stond ook wel een erg goeie wind! Het doet me deugd dat ook jij nu als AlO bij de heelkunde werkzaam bent. Van student-assistent tot collega, paranymf en vriend.

Drs Marc Daemen, beste Marc. Jouw sollicitatie gesprek inzake het student-assistent schap zal me nog lang heugen. Als enige had jij geen $\mathrm{CV}$ meegestuurd daar je "toch niets had om hierin te vermelden". Je had voor de sollicitatie nieuwe schoenen gekocht, maar was in alle opwinding vergeten je veters te strikken. En eigenlijk wilde je helemaal geen algemeen chirurg worden maar neurochirurg. Toch had ik op de een of andere manier al snel door dat jij de beste kandidaat was, een keuze waar ik nooit spijt van heb gehad. Je hebt niet alleen veel werk voor me verzet maar bleek daarnaast ook nog eens aangenaam gezelschap. Loyaliteit, oprechtheid en collegialiteit heb jij hoog in je vaandel staan. Het doet me deugd dat ook jij nu als onderzoeker bij de Heelkunde werkzaam bent. Gelukkig had je dit keer wel heel wat om in je $\mathrm{CV}$ te vermelden.

Drs Ellen Rouwet, beste Ellen. Jouw meest kenmerkende eigenschappen zijn werklust, nauwgezetheid en gedrevenheid. Met name dank zij deze eigenschappen heb jij een belangrijke bijdrage geleverd aan een deel van de dierexperimentele onderzoeken. Samen met Marc heb je diverse prijzen in de wacht weten te slepen. Het doet me genoegen om te zien hoe je in de afgelopen jaren bent geevolueerd. Ik heb je nu zelfs al 2 keer dronken gezien: ik heb werkelijk nooit geweten dat een toilet in een Munchens bierhuis zich leent voor een verblijf van enkele uren! Gelukkig waren we je niet vergeten toen we de thuisreis aanvaardden. Ook jij bent nu werkzaam als arts-onderzoekster bij de heelkunde; het trias is weer compleet.

Prof Hoeks, beste Arnold, de in hoofdstuk 9 beschreven experimenten zijn uitgevoerd met het door jou ontwikkeldle vessel wall Doppler tracking systeem. Volledig belangeloos heb jij niet alleen dit zeer fraaie maschien ter beschikking gesteld maar ook het manuscript kritisch nagelezen. Jean Willigers, beste Jean, alhoewel niet direct betrokken bij het onderzoek heb jij me regelmatig terzijde gestaan in geval van calamiteiten op technisch vlak. 
Prof Dr Kootstra, bedankt woor de mogelijkheid om mijn promotie-onderzoek op Uw afdeling te kuninen verwezenlijken. De collega-assistenten heelkunde bedankt voor hun kameraadschap en voor het ontzien gedurende de afrondingsfase van mijn proefschrift.

De leden van de beoordelingsconmissie, Prof Dr. HAJ Struijker Boudier (voorzitter), Prof. Dr. RIA Goris, Prof. Dr. ME Sluijter en Prof. Dr. F Spaans wil ik bedanken voor de beoordeling van het manuseript. I would like to thank Prof. Tooke for reviewing this thesis.

Een extra woord van dank aan Prof. Goris. Al weer sinds enige tijd draagt $U$ er zorg voor dat wij in het Maastrichtse op de hoogte worden gesteld indien humaan materiaal voor onderzoek ter beschikking komt. Diverse keren was dit het geval in het Radbout ziekenhuis. Iedere keer opnieuw zijn wij door $U$ en $U w$ medewerkers bijzonder gastvrij ontvangen waarvoor mijn dank. Met genoegen heb ik diverse malen met $U$ van gedachten mogen wisselen omtrent pathofysiologische mechanismen welke aan RSD ten grondslag liggen. Uw onderzoek en patientenzorg op dit vlak staan in Nederland op eenzame hoogte. Het is dan ook mede hieraan te danken dat het ziektebeeld nationaal en internationaal meer in de belangstelling is komen te staan, hetgeen herkenning en erkenning zeker ten goede is gekomen. Ik hoop dat onze samenwerking nog lang moge blijven bestaan.

De diverse microcirculatoire onderzoekers van de afdelingen fysiologie (Miriam, Berrie, Jacqueline, Martijn en Selma), farmacologie (Jos, Ferdie) en interne geneeskunde (Boy) hebben allen op hun eigen wijze bijgedragen aan een prettige sfeer in de onderzoekslaboratoria en nog meer tijdens congressen. Sabrina van Velzen en Rinus Alewijnse bedankt voor hand en spandiensten ten behoeve van zowel humane alsook dierexperimentele studies. Huub Jussen heeft zorg gedragen voor kleine en grote onderhoudsbeurten van de microscoop-opstelling.

Yvonne Daemen, diverse malen heb jij mij op voortvarende wijze met hand en spandiensten terzijde gestaan. Het doet mij genoegen dat we jou hebben kunnen strikken om het $10 \mathrm{de}$ SEOHS, hetwelke dit jaar in Maastricht wordt gehouden, secretarieel te ondersteunen. Ook dank aan Miranda Thimister voor secretariele ondersteuning

De afdeling CPV onder leiding van Ton van de Boogaard wordt bedankt voor het verzorgen van de ratten alsmede voor het verstrekken van diverse nuttige adviezen.

De dames van het vaatlab, Ineke, Yvonne, Laura en Monique, alhoewel alweer enige tijd geleden heeft een groot deel van het patient-gebonden onderzoek op de funktie-afdeling van de vaten plaatsgevonden. Dank voor het bijhouden van de afspraken en voor het creeren van een prettige sfeer; met name de Sinterklaasvieringen heb ik als zeer gezellig ervaren.

Geachte oudleden van het Maastrichtse Heerendispuut Prometheus: jullie boden mij de entourage waarin ik zo nu en dan helemaal los kon komen van promotie- en heelkundige opleidings-perikelen. Wat whiskey, sigaren en bijzonder aangenaam gezelschap al niet vermogen!

Last but certainly not least, Gonnie, van veel was te weinig. Alles sal beter wordt. 


\section{Publications (full papers)}

1. Reflex sympathetic dystrophy: evolution of microcirculatory disturbances in time. Kurvers HAJM, Jacobs MJHM, Beuk RJ, Wildenberg van den FAJM, Kitslaar PJEHM, Slaaf DW, Reneman RS. Pain, 60, 333-340, 1995.

2. Reflex sympathetic dystrophy, result of autonomic denervation? Kurvers HAJM, Jacobs MJHM, Beuk RJ, Wildenberg van den FAJM, Kitslaar PJEHM, Slaaf DW, Reneman RS. Clinical Science, 87, 663-669, 1994.

3. The influence of local skin heating and reactive hyperenia on skin blood flow abnormalities in patients with reflex sympathetic dystrophy, Kurvers HAJM, Jacobs MJHM, Beuk RJ, Wildenberg van den FAJM, Kitslaar PJEHM, Slaaf DW, Reneman RS. European Journal of Clinical Investigation, 25, 346-352, 1995.

4. The spinal component to skin blood flow abnomalities in reflex sympathetic dystrophy. Kurvers HAJM, Jacobs MJHM, Beuk RJ, Wildenberg van den FAJM, Kitslaar PJEHM, Slaaf DW, Reneman RS. Archives of Neurology, 53, 58-65, 1996.

5. Reflex sympathetic dystrophy; does sympathetic dysfunction originate from peripheral neuropathy? Kurvers HAJM, Hofstra L, Jacobs MJHM, Daemen MARC, Wildenberg van den FAJM, Kitslaar PJEHM, Slaaf DW, Reneman RS. Surgery, 119, 288-296, 1996.

6. Skin blood flow abnormalities in a rat model of neuropathic pain: result of decreased sympathetic vasoconstrictor outflow? Kurvers HAJM, Tangelder GJ, De Mey JGR, SlaafDW, Beuk RJ, Wildenberg van den FAJM, Kitslaar PJEHM, Reneman RS, Jacobs MJHM. Journal of the Autonomic Nervous System, 63, 19-29, 1997.

7. Skin blood flow disturbances in the contralateral limb in a peripheral mononeuropathy in the rat. Kurvers HAJM, Tangelder GJ, De Mey JGR, Slaaf DW, Wildenberg van den FAJM, Kitslaar PJEHM, Reneman RS, Rouwet EV, Iacobs MJHM. Neuroscience, 74, (3), 935-943, 1996.

8. The influence of partial nerve injury in the rat on efferent function of sympathetic and antidromically acting sensory nerve fibers. Kurvers HAJM, Tangelder GI, De Mey JGR, Reneman RS, Slaaf DW, Rouwet EV, Wildenberg van den FAJM, Kitslaar PJEIM, Jacobs MJHM. Journal of Trauma, 41, 981-988, 1996.

9. Reflex sympathetic dystrophy; a review. Kurvers HAJM. Journal of the Peripheral Nervous System. (In press)

10. Sympathetic dysfunction in a rat model of partial nerve injury consists of denervationinduced supersensitivity to catecholamines. Kurvers HAJM, Tangelder G.J, De Mey JGR, Slaaf DW, Beuk RJ, Wildenberg van den FAJM, Kitslaar PJEHM, Slaaf DW, Reneman RS, Jacobs MJHM. (Submitted)

11. Microcirculatory disorders in reflex sympathetic dystrophy. Kurvers HAJM, Jacobs MJHM, Beuk RJ, Wildenberg van den FAJM, Slaaf DW, Kitslaar PJEHM, Reneman RS. The Pain Clinic, 8, (1), 15-25, 1995. 
12. Microcirculatory abnormalities in reflex sympathetic dystrophy and an animal model of neuropathic pain; hypersensitivity to catecholamines consequent to autonomic denervation? Kurvers HAJM, Stassen F, Jacobs MJHM, Daemen MARC, Rouwet EV, Wildenberg van den FAJM, Kitslaar PJEHM, Slaaf DW, Reneman RS, Demey JGR. NVBP-pijnbulletin, 2, 2-4, 1994.

13. Neue aspekte beim post traumatischen dystrophie syndrom? Wildenberg van den FAJM, Kurvers HAJM, Jacobs MJHM, Eggink GJ, Bulstra S, Sluyter M, Dingemans W. Unfallchirurg, 97, 485-490, 1994.

14. Spinal cord stimulation and reflex sympathetic dystrophy. Spincemaille GH, Barendse G, Rouwet EV, Kurvers HAJM, Adang EMM, Kleef van M, Dingemans W, Wildenberg van den FAJM. The Pain Clinic, 8, (2), 155-160, 1995.

15. Microcirculatoire aspecten van een neuropathisch pijnsyndroom: de rol van het sympathische zenuwstelsel. Daemen MARC, Kurvers HAJM, Kitslaar PJEHM, Wildenberg van den FAJM, Beuk RJ, Rouwet EV, Jacobs MJHM, Reneman RS. Tijdschrift voor vaatdiagnostiek, 3, 9-13, 1995.

16. Spinal Cord Stimulation in de behandeling van reflex sympathische dystrofie. Rouwet EV, Spincemaille GH, Barendse G, Kleef van M, Adang EMM, Kurvers HAJM, Daemen MARC, Wildenberg van den FAJM. Nederlands Tijdschrift voor Pijn en Pijnbestrijding, 1, 8-11, 1996.

17. Denervation-induced adrenoceptor supersensitivity in an experimental animal model of reflex sympathetic dystrophy; functional studies. Kurvers HAJM, Stassen, Daemen MARC, SlaafDW, Wildenberg van den FAJM, Kitslaar PJEHM, Reneman RS, De Mey JGR. Acta Orthopaedica Belgica. (In press)

18. Motor dysfunction in an experimental animal model of reflex sympathetic dystrophy; bilateral motor denervation. Bullens P, Daemen MARC, Kurvers HAJM, Freling G, Kitslaar PJEHM, Wildenberg van den FAJM. Acta Orthopaedica Belgica. (In press)

19. Neurogenic inflammation in an experimental animal model of reflex sympathetic dystrophy; in vivo and in vitro assessment. Daemen MARC, Kurvers HAJM, Bullens P, Barendse G, Kleef van M, Wildenberg van den FAJM. Acta Orthopaedica Belgica. (In press)

20. Neurogenic inflammation in an animal model of neuropathy. Daemen MARC, Kurvers HAJM, Kitslaar PJEHM, Slaaf DW, Bullens P, Wildenberg van den FAJM. (submitted)

21. Sympathetic dysfunction in RSD patients consists of denervation-induced supersensitivity to catecholamines. Kurvers HAJM, Tangelder GJ, De Mey JGR, Slaaf DW, Beuk RJ, Wildenberg van den FAJM, Kitslaar PJEHM, Slaaf DW, Reneman RS, Jacobs MJHM. (Submitted)

22. Ipsilateral motor denervation and muscle fibre type redistribution in a rat model of neuropathic pain. Daemen MARC, Kurvers HAJM, Bullens PHJ, Freling G, Slaaf DW, Kitslaar PJEHM, Wildenberg van den FAJM. (Submitted) 


\section{Abstracts (international congresses)}

1. Capillary microscopy, laser doppler fluxmetry and transcutaneous oxymetry in the evaluation of skin microcirculation in Sudeck's dystrophy. 5th World Congress for Microcirculation, Lounsville, USA, 31 augustus
-5 september 1991 .

2. Sympathetic dysfunction in Sudeck's dystrophy assessed by Vessel Wall Echo Tracking. 26th unnual scientific meeting of the European Society for Clinical Inwestigation, Viema, Austria, 1-4 april 1992.

3. Capillary microscopy and laser Doppler fluxmetry in the assessment of bilateral involvement in Sudeck's dystrophy, 17 th European Conference on Microcirculation, London, UK, juli 1992.

4. Edema formation in reflex sympathetic dystrophy, a result of a decline in vasoconstrictive capacity? 16 th World Congress of the Intemational Union of Angiology, Parijs, 13-18 september 1992.

5. Bilateral sympathetic involvement in Sudeck's dystrophy assessed by vessel wall doppler tracking, 16th World Congress of the International Union of Angiology, Parijs, $13-18$ september 1992.

6. Microcirculatory investigation in the assessment of bilateral involvement in Sudeck's dystrophy, 16 th World Congress of the International Union of Angiology, Parijs, 13-18 september 1992.

7. The influence of sympathetic dysfunction on compliance of the brachial artery in Sudecks dystrophy, 16th World Congress of the International Union of Angiology, Parijs, 13-18 september 1992.

8. Microcirculation and reflex sympathetic dystrophy: Inwited lecture, 6th international pain symposium Delft Rotterdam-Tokyo on "sympathetic nervous system and pain". June 26, 1993.

9. Effect of $\alpha$-adrenoceptor antagonists on mechanical allodynia in a rat model of reflex sympathetic dystrophy. Pharmacology of Adrenoceptors, King of Prussia, USA, 2 i-23 julli 1994.

10. Skin blood flow abnormalities in a rat model of neuropathic pain: result of a decrease instead of an increase in efferent sympathetic nerve discharge? 18 th European Conference on Microcirculation, Rome, Italie, 4-9 september 1994.

11. Reflex sympathetic dystrophy: result of autonomic denervation? 18th European Conference on Microcirculation, Rome, Italie, 4-9 september 1994.

12. Chronic constriction of the sciatic nerve in the rat results in autonomic denervation and consequent hyperreactivity to catecholamines of resistance arteries. 18 th European Conference on Microcirculation, Rome, Italie, 4-9 september 1994.

13. Microcirculation in reflex sympathetic dystrophy. Combined meeting of the Dutch Pain Society and the Pain Society of Britain and Ireland, Amsterdam, 22-24 september 1994.

14. Loose ligation of the rat sciatic nerve induces autonomic denervation and hypersensitivity to catecholamines. Ist scientific meeting of the European LASP-chapters "Pain in Europe", Verona (Italie), 18-21 mei 1995.

15. Involvement of sympathetic and antidromic mechanisms in skin blood flow abnormetlities in a neuropathic pain model in the rat. 36th World Congress of Surgery, Lissabon, Portugal, august 27th - septenber 2 nd 1995.

16. Autonomic denervation and hypersensitivity to catecholamines in an animal model of reflex sympathetic dystrophy. Brussels International Upper Extremity Symposium; reflex sympathetic dystrophy * Brussels, Belgium. April 29-30, 1996.

17. Loose ligation of the rat sciatic nerve induces autonomic denervation and hypersensitivity to catecholamines. 8 th world congress on pain, Vancouver, British Columbia, Canada, August 17-22, 1996.

18. Neurogenic inflammation in a model of neuropathic pain; in vitro assessment. 8 th world congress on pain, Vancouver, British Columbia, Canada, August 17-22, 1996. 


\section{CURRICULUM VITAE}

Henricus Anna Johannes Maria Kurvers was born on July 31st, 1964, in Sittard, The Netherlands. He graduated in 1982 from the "bisschoppelijk college" in Sittard. He continued his study at the medical faculty of the university of Maastricht. After receiving his doctoral degree in 1986, he received his medical degree in 1989. During his study, he worked as a research assistent at the department of arthopaedic surgery of the university hospital Maastricht and as a paramedic on the emergency assistant team in Heerlen. At the end of his study he worked, for a period of 6 months, as a resident at the department of orthopedic traumatology of the Siriraj Hospital, Bangkok; Thailand. From 1989 until 1991, he fullfilled his military service as a resident in orthopedic surgery, military hospital Dr. A. Mathijsen, Utrecht. From 1991 until 1995 he was a research fellow (AIO) at the department of general surgery (university hospital Maastricht and cardiovascular research institute Maastricht). During this period the work contained in this thesis was accomplished under the supervision of Prof. Dr. PJEHM Kitslaar, Prof. Dr. MJHM Jacobs, and Dr. FAJM van den Wildenberg from the university hospital Maastricht and of Prof. Dr. RS Reneman and Dr. DW Slaaf from the cardiovascular research institute Maastricht. In 1995 he started his surgical training in the University Hospital Maastricht (head Prof. Dr. G. Kootstra).

Financial support for the publication of this thesis was generously provided by:

Klinerva BV

West Meditec

Howmedica Nederland

WL Gore and Associates BV

TD Medical BV

Pie Medical Benelux BV

Schering-Plough BV

SmithKline Beecham Farma BV

Zambon Nederland BV

Byk Nederland BV

Boehringer Ingelheim BV

Bristol-Myers Squibb BV

Glaxo Wellcome BV

Gambro medische apparaten BV 